
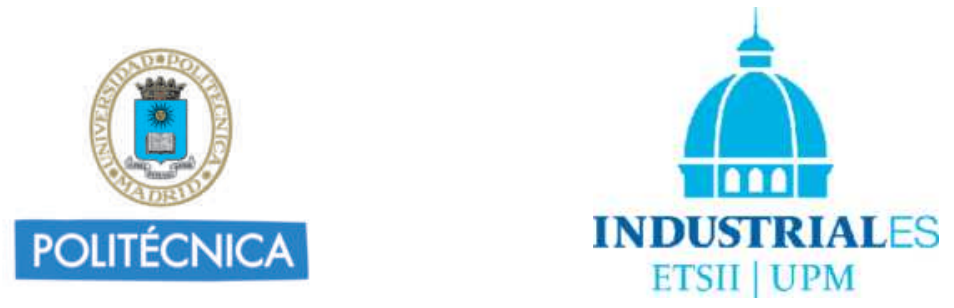

Universidad Politécnica de Madrid

Escuela Técnica Superior de Ingenieros Industriales

\title{
Learning-Based Perception, Control, And NAVigation for Autonomous Missions in Aerial RoвотICS
}

\author{
Ph.D. Thesis
}

\section{Carlos Sampedro Pérez}

M.Sc. in Automation and Robotics 


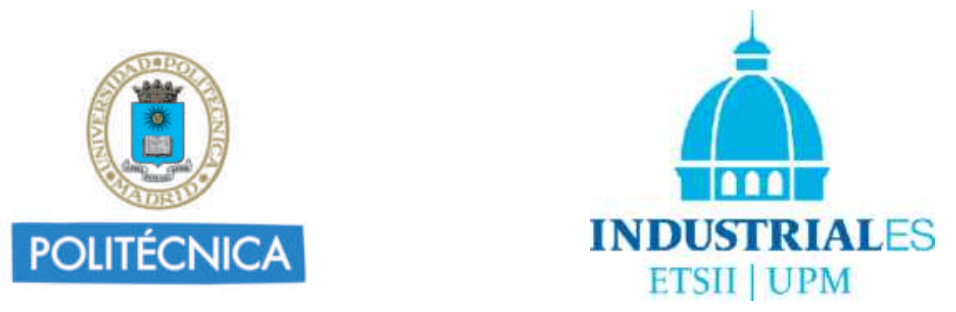

Departamento de Automática, Ingeniería Eléctrica y Electrónica e Informática Industrial

Escuela Técnica Superior de Ingenieros Industriales

Universidad Politécnica de Madrid

\title{
Learning-Based Perception, Control, AND Navigation for Autonomous Missions in Aerial RoBotics
}

\author{
A thesis submitted for the degree of \\ Doctor of Philosophy in Automation and Robotics
}

Author:

Carlos Sampedro Pérez

M.Sc. in Automation and Robotics

Director:

Pascual Campoy Cervera

Prof. Dr. in Automation and Robotics

Madrid, December 2019 

Título:

Learning-Based Perception, Control, and Navigation for Autonomous

Missions in Aerial Robotics

\author{
Autor: \\ Carlos Sampedro Pérez \\ M.Sc. in Automation and Robotics
}

Tribunal nombrado por el Magfco. y Excmo. Sr Rector de la Universidad Politécnica de Madrid el día.........de..... de 2019

\title{
Tribunal
}

Presidente :
Secretario :
Vocal :
Vocal :
Vocal :
Suplente :
Suplente :

Realizado el acto de lectura y defensa de la tesis el día de de 2019. En la Escuela Técnica Superior de Ingenieros Industriales de la Universidad Politécnica de Madrid

Calificación de la Tesis:

El Presidente:

Los Vocales:

El Secretario: 



\section{Acknowledgements}

I would like to express my gratitude to my supervisor Prof. Pascual Campoy, for his support and guidance during the realization of this work. I also would like to thank Prof. Sergio Dominguez and Prof. Martin Molina for their advice and help during the realization of this thesis.

Since I consider the $\mathrm{PhD}$ as a process not only for sharing knowledge, but also for sharing life experiences, I would like to express my sincere gratitude to the fantastic group of people, scientist, and engineers (all in one), who compose the Computer Vision and Aerial Robotics (CVAR) group. I greatly thank Ramon Suarez for his brilliant ideas and solutions to any problem, Adrian Carrio for his perseverance and willingness to investigate new solutions, Hriday Bavle for his positivity, predisposition to work, and his magnificent capabilities for flying any flying artifact, Alejandro Rodriguez, my partner in reinforcement learning, Javier Rodriguez for his intelligent conversations about computer vision and machine learning problems, Lu Liang for his good sense of humor and availability for work, and Zorana Milosevic for her friendship. I would like to thank also Prof. Paloma de la Puente for her support, ideas and contributions.

Additionally, I would like to mention and especially thank past members of the CVAR group, who have helped in the learning process of my PhD. I would like to thank Jose Luis Sanchez for his scientific rigor and good leadership abilities, Jesus Pestana, for his constant contribution of stimuli in any scientific subject, and to my I2L fellows: Carol Martinez, Aneesh Chauhan, and Jean François Collumeau for their help and guidance for my personal scientific growing. In addition, I would like to especially thank Miguel Olivares and Ivan Mondragon for the revision of this document.

I also want to thank the people from the Autonomous Systems Technologies Research \& Integration Laboratory (ASTRIL) group in the Arizona State University where I conducted my research stay. I really thank Prof. Srikanth Saripalli, for giving me the opportunity to conduct a research stay under his supervision, and to my colleagues Ben, Aravindhan, and especially Sai Vemprala for his intelligent conversations.

It is my pleasure to thank the Universidad Politécnica de Madrid for funding my pre-doctoral contract and make this scientific work possible. In addition, I would also like to thank my former professors Miguel Hernando, Diego Rodriguez-Losada, Pablo San Segundo and Carlos Platero, who boosted my interest in science, programming, and computer vision. Their ability to transmit knowledge and stimulate student interest, have undoubtedly contributed to the development of this thesis.

Finally, I would like to express my most special gratitude to my friends and the members of my family for their interest in my work, and especially to my life partner, Ana, for her constant support and understanding during this long journey. Her positivity and constant support have undoubtedly been the greatest contribution to the work presented here. 



\section{Resumen}

El diseño de algoritmos para sistemas robóticos móviles representa un desafío importante, ya que estos sistemas deben operar en una amplia gama de escenarios no estructurados donde se requiere que el robot interactúe con el entorno de manera eficiente. Esta interacción puede ser crítica para los sistemas robóticos aéreos, ya que una pequeña falla (por ejemplo, tocar un obstáculo durante la navegación) puede comprometer la seguridad de todo el sistema. Para lograr comportamientos complejos que puedan manejar el amplio espectro de situaciones que pueden ocurrir durante la ejecución de una misión, la comunidad de investigación está evolucionando hacia el desarrollo de soluciones basadas en el aprendizaje y de inspiración biológica. En esta dirección, la inteligencia artificial y, más concretamente, los modelos de aprendizaje automático, están adquiriendo una importancia significativa debido a su versatilidad para trabajar en una amplia gama de condiciones. Estos modelos son capaces de aprender directamente de los datos, evitando el uso de heurísticas hechas a mano, que son difíciles de adaptar a la amplia gama de escenarios donde deberían operar las plataformas robóticas. En los últimos años, el crecimiento de los recursos computacionales ha permitido el uso de técnicas más complejas basadas en el aprendizaje. En esta dirección, las técnicas de aprendizaje profundo se están investigando ampliamente en la comunidad científica, ya que pueden proporcionar modelos no lineales capaces de aprender comportamientos complejos y robustos.

En esta dirección, esta tesis presenta diferentes soluciones basadas en el aprendizaje, principalmente basadas en redes neuronales profundas, integradas en capas esenciales en una arquitectura robótica aérea, como la percepción, el control y la navegación. En este trabajo, la percepción se concibe como la tarea de usar datos basados en la visión para el reconocimiento de objetos, que se aborda en esta tesis mediante el diseño de algoritmos tradicionales basados en el aprendizaje y redes más sofisticadas que tienen como base las redes neuronales convolucionales. Además, se agrega un nivel más alto de inteligencia mediante el diseño de modelos supervisados y semi-supervisados, usando redes siamesas y autoencoders, para detectar anomalías o estados anormales en los objetos reconocidos anteriormente. Con respecto a las tareas de control y navegación, utilizamos algoritmos de aprendizaje por refuerzo que se entrenan de manera eficiente para realizar tareas de visual servoing y navegación reactiva. Finalmente, proponemos un sistema versátil de planificación de misiones capaz de coordinar diferentes subsistemas para realizar misiones totalmente autónomas utilizando diferentes plataformas aéreas robóticas.

La mayoría de los algoritmos presentados en esta tesis han sido validados en experimentos de simulación y de vuelo real utilizando diferentes plataformas robóticas aéreas. Los resultados obtenidos en los diferentes experimentos realizados a lo largo de esta tesis demuestran las capacidades robustas proporcionadas por los sistemas basados en el aprendizaje. Estos algoritmos han demostrado que aprenden comportamientos complejos que pueden ayudar en situaciones donde los algoritmos tradicionales pueden presentar limitaciones. 



\section{Abstract}

The design of algorithms for mobile robotic systems represents a significant challenge since these systems should operate in a wide range of unstructured scenarios where the robot is required to interact with the environment efficiently. This interaction can become critical for aerial robotic systems since a small failure (e.g., touching an obstacle during navigation) can compromise the security of the entire system. In order to achieve complex behaviors able to handle the wide spectrum of situations that can occur during the execution of a mission, the research community is evolving towards the development of biologically-inspired and learning-based solutions. Artificial intelligence, and more concretely machine learning models, are gaining significant importance owing to their versatility for working in the wide range of conditions where mobile robotic systems have to operate. These models are capable of learning directly from data, avoiding the use of handcrafted heuristics. In recent years, the growth of computational resources has permitted the usage of more complex learning-based techniques. In this direction, deep learning techniques are being widely researched in the scientific community since they can provide non-linear models capable of learning complex and robust behaviors.

To this aim, this thesis presents different learning-based solutions, primarily based on deep neural networks, integrated into essential layers in an aerial robotic architecture, such as perception, control, and navigation. In this work, perception is conceived as the task of using vision-based data for object detection, which is addressed in this thesis by designing traditional learning-based algorithms and more sophisticated fully convolutional networks. In addition, a higher level of intelligence is added by designing supervised and semisupervised models, using siamese networks and autoencoders, for detecting anomalies or abnormal states in the objects previously detected. Regarding the control and navigation tasks, we use deep reinforcement learning algorithms which are efficiently trained for visual servoing and target-driven reactive navigation. Finally, we propose a versatile mission planning system capable of coordinating different subsystems for performing fullyautonomous missions in dynamic and unstructured scenarios.

Most of the algorithms presented in this thesis have been validated in simulation and real-flight experiments using different aerial robotic platforms. The results obtained in the different experiments conducted throughout this thesis demonstrate the robust capabilities provided by learning-based systems. These algorithms have proven to learn complex behaviors which can help in situations where traditional algorithms can present limitations. 



\section{Acronyms}

AI: Artificial Intelligence

APF: Artificial Potential Field

ArUco: Augmented Reality from University of Cordoba

AUC: Area Under the Curve

CAE: Convolutional Autoencoder

CNN: Convolutional Neural Network

CPU: Central Processing Unit

DDPG: Deep Deterministic Policy Gradients

DOF: Degree Of Freedom

DRL: Deep Reinforcement Learning

EKF: Extended Kalman Filter

EWMA: Exponentially Weighted Moving Average

FOV: Field Of View

FPS: Frames Per Second

FSM: Finite-State Machine

GAN: Generative Adversarial Network

GNSS: Global Navigation Satellite System

GPU: Graphics Processing Unit

HOG: Histogram of Oriented Gradients

HSV: Hue Saturation Value

IBVS: Image-Based Visual Servoing

IMAV: International Micro Air Vehicle Conference and Competition

IMU: Inertial Measurement Unit

IoU: Intersection over Union

JCR: Journal Citation Reports

LBP: Local Binary Pattern

LIDAR: Light Detection And Ranging

MAE: Mean Absolute Error

mAP: mean Average Precision

MAV: Micro Aerial Vehicle

MDP: Markov Decision Process

Mocap: Motion Capture System

MPC: Model Predictive Control 
MSE: Mean Squared Error

NAF: Normalized Advantage Functions

PID: Proportional-Integral-Derivative

P $n$ P: Perspective- $n$-Point

RANSAC: Random Sample Consensus

RBF: Radial Basis Function

RGB: Red Green Blue

RL: Reinforcement Learning

RMS: Root Mean Square

ROC: Receiver Operating Characteristic

ROI: Region Of Interest

ROS: Robot Operating System

RRT: Rapidly-exploring Random Tree

SAR: Search And Rescue

SCAE: Siamese Convolutional Autoencoder

SCNN: Siamese Convolutional Neural Network

SGD: Stochastic Gradient Descent

SLAM: Simultaneous Localization And Mapping

SVM: Support Vector Machine

UAV: Unmanned Aerial Vehicle

UML: Unified Modeling Language

USAR: Urban Search And Rescue 


\section{Contents}

\begin{tabular}{lll}
\hline Acknowledgements & V \\
\hline & &
\end{tabular}

Resumen VII

\begin{tabular}{ll}
\hline Abstract & IX
\end{tabular}

\begin{tabular}{|ll}
\hline Acronyms & XI
\end{tabular}

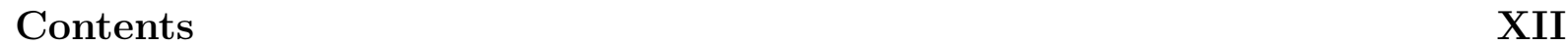

$\begin{array}{ll}\text { List of Figures } & \text { XVII }\end{array}$

$\begin{array}{ll}\text { List of Tables } & \text { XXI }\end{array}$

$\begin{array}{lll}1 & \text { Introduction } & 1\end{array}$

1.1 Learning-based algorithms for robotics . . . . . . . . . . . . . . . . . 1

1.2 Motivation of the Thesis . . . . . . . . . . . . . . . . . . . . . 3

1.3 Objectives of the Thesis . . . . . . . . . . . . . . . . . . 4

1.4 Methodology $\ldots \ldots \ldots \ldots \ldots \ldots \ldots$

1.5 Contributions and Dissertation Outline . . . . . . . . . . . . . . 6

1.6 List of Publications . . . . . . . . . . . . . . . . . . . . . . . . . . . 9

1.6 .1 Journal Publications . . . . . . . . . . . . . . . . . . . 9 9

1.6.2 Publications in International Peer-Reviewed Conferences . . . . . . 9

2 Object Detection and Segmentation 11

2.1 Introduction . . . . . . . . . . . . . . . . . . . . . . 11

2.2 Related Work . . . . . . . . . . . . . . . . . . . . . . . . . . . . . . 13

2.3 Algorithms based on Region Proposals . . . . . . . . . . . . . . . . . . . . 16

2.4 Algorithms based on Fully Convolutional Networks . . . . . . . . . . . . . 18

$2.5 \quad$ Experiments and Results . . . . . . . . . . . . . . . . . . . . . 20

2.5 .1 Experimental Setup . . . . . . . . . . . . . . . . . . . 20

2.5 .2 Experimental Results in Electric Tower Detection . . . . . . . . . . 20

2.5.3 Experimental Results in Electric Insulator Segmentation . . . . . . 26

2.5.4 Experimental Results in Helipad Detection . . . . . . . . . . . . . . 34

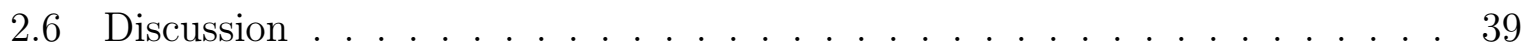

2.7 Conclusions $\ldots \ldots \ldots \ldots$ 
Contents

$\begin{array}{llr}3 & \text { Anomaly Detection } & 47\end{array}$

3.1 Introduction . . . . . . . . . . . . . . . . . . . . . . . . 47

3.2 Related Work . . . . . . . . . . . . . . . . . . . . . . . . . . . . . . . . . 49

3.3 System Description . . . . . . . . . . . . . . . . . . . . . . . . 51

3.4 Learning-based Anomaly Detection . . . . . . . . . . . . . . . . . . . 52

3.4 .1 Supervised Anomaly Detection . . . . . . . . . . . . . 52

3.4 .2 Semi-Supervised Anomaly Detection . . . . . . . . . . . . . . . . 58

3.5 Experiments and Results . . . . . . . . . . . . . . . . . . . . . . 59

3.5.1 Experimental Setup . . . . . . . . . . . . . . . . . . 60 60

3.5.2 Diagnosis of absent disc units . . . . . . . . . . . . . . . . . 60

3.5.3 Diagnosis of damaged disc units . . . . . . . . . . . . . . . . . 63

3.5.4 System evaluation results . . . . . . . . . . . . . . . . . 71

3.6 Discussion . . . . . . . . . . . . . . . . . . . . . . . . . . . . . 75

3.7 Conclusions $\ldots \ldots \ldots \ldots$

\begin{tabular}{|lll}
\hline & Image-Based Visual Servoing & 81
\end{tabular}

4.1 Introduction . . . . . . . . . . . . . . . . . . . . . 81

4.2 Related Work . . . . . . . . . . . . . . . . . . . . . . . . . . . . . . . . . . 82

4.3 Image-Based Visual Servoing Controllers . . . . . . . . . . . . . . . . . . . 83

4.3 .1 Classic and Partitioned IBVS Controllers . . . . . . . . . . . . . . . 83

4.3 .2 IBVS through Reinforcement Learning controller . . . . . . . . . . 84

4.4 Experiments and Results $\ldots \ldots \ldots \ldots \ldots$. . . . . . . . . . . . . . . 89

$4.4 .1 \quad$ Experimental setup . . . . . . . . . . . . . . . . . . . . . . 89

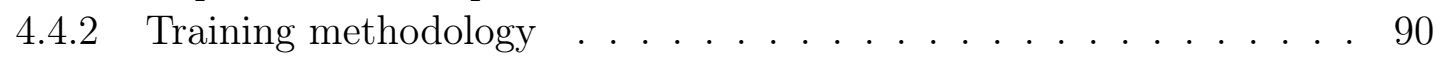

$4.4 .3 \quad$ Simulation Experiments . . . . . . . . . . . . . . . . . . . . . . . . 93

$4.4 .4 \quad$ Real-flight Experiments . . . . . . . . . . . . . . . . . . . . . . . 98

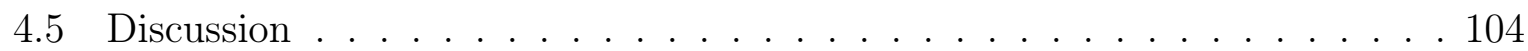

4.6 Conclusions $\ldots \ldots \ldots$

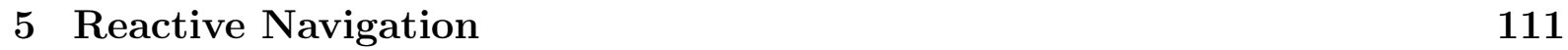

5.1 Introduction . . . . . . . . . . . . . . . . . . . . 111

5.2 Related Work . . . . . . . . . . . . . . . . . . . . . . . . . 112

5.3 Target-driven Reactive Navigation through RL . . . . . . . . . . . . . . . . 114

5.4 Experiments and Results . . . . . . . . . . . . . . . . . . . . . . . . . . . 118

$5.4 .1 \quad$ Experimental setup . . . . . . . . . . . . . . . . . . . . . . . 118

5.4 .2 Training methodology . . . . . . . . . . . . . . . . . . . . 119

$5.4 .3 \quad$ Simulation Experiments . . . . . . . . . . . . . . . . . . . 120

5.4 .4 Real-flight Experiments . . . . . . . . . . . . . . . . 125

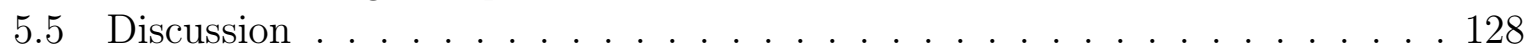

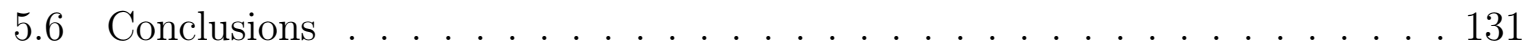

\begin{tabular}{llr}
\hline & Mission Planning & 133
\end{tabular}

6.1 Introduction . . . . . . . . . . . . . . . . . . . . . . 133

6.2 Related Work . . . . . . . . . . . . . . . . . . . . . . . . . . . 135

6.3 Mission Planning Architecture . . . . . . . . . . . . . . . . . . . . 137 
6.3 .1 Global Mission Planner . . . . . . . . . . . . . . . . . . . . . . . 137

6.3 .2 Agent Mission Planner . . . . . . . . . . . . . . . . . . . . . . . . . 141

6.4 Additional Components . . . . . . . . . . . . . . . . . . . . . . 143

$6.4 .1 \quad$ Planning System . . . . . . . . . . . . . . . . . . . . . . . . . . . . . . . . . . . . . . . . . . . . .

6.4 .2 Executive System . . . . . . . . . . . . . . . . . . . . . . . . . 144

$6.4 .3 \quad$ Situation Awareness System . . . . . . . . . . . . . . . . . . . . . . 145

6.4 .4 Feature Extraction System . . . . . . . . . . . . . . . . . . 149

6.4 .5 Motor System . . . . . . . . . . . . . . . . . . . . . . 150

6.5 Experiments and Results . . . . . . . . . . . . . . . . . . . . 152

6.6 Experimental Results using Swarms of UAVs . . . . . . . . . . . . . . . . . 152

$6.6 .1 \quad$ Experimental Setup . . . . . . . . . . . . . . . . . 153

$6.6 .2 \quad$ Simulation Experiments . . . . . . . . . . . . . . . . . . . 153

6.6 .3 Real-flight Experiments . . . . . . . . . . . . . . . . 156

6.7 Experimental Results in SAR missions . . . . . . . . . . . . . . . 157

$6.7 .1 \quad$ Experimental Setup . . . . . . . . . . . . . . . . . . 157

6.7 .2 Simulation Experiments . . . . . . . . . . . . . . . . . . . . 159

6.7 .3 Real-flight Experiments . . . . . . . . . . . . . . . . . . . . . . . 159

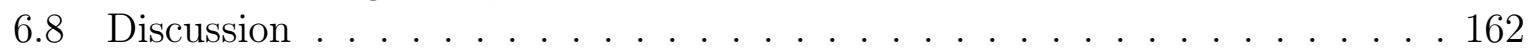

6.9 Conclusions . . . . . . . . . . . . . . . . . . . . . . . 166

$\begin{array}{lll}7 & \text { Conclusions and Future Work } & 167\end{array}$

7.1 Conclusions . . . . . . . . . . . . . . . . . . 167

7.2 Future Work . . . . . . . . . . . . . . . . . . . . . . . . . . . . . . . . . . . . . 168

\begin{tabular}{lr}
\hline Appendices & 169
\end{tabular}

\begin{tabular}{|lr}
\hline A Deep Learning Background & 171
\end{tabular}

A.1 Deep Neural Networks . . . . . . . . . . . . . . . . . . . . . . . . . . . 171

A.1.1 Basics in Convolutional Neural Networks . . . . . . . . . . . . . . . 171

A.1.2 Activation Functions . . . . . . . . . . . . . . . . . . . 172

A.1.3 Optimization . . . . . . . . . . . . . . . . . . . . 174

A.2 Deep Reinforcement Learning _ . . . . . . . . . . . . . . . . . . 177

\begin{tabular}{lr}
\hline B Additional Object Detection Results & 181
\end{tabular}

B.1 Image Dataset . . . . . . . . . . . . . . . . . . . . . . . . . . 181

B.2 Training Methodology and Evaluation . . . . . . . . . . . . . . . . 182

\begin{tabular}{lr}
\hline C Scientific Dissemination & 189
\end{tabular}

C.1 Journal Publications . . . . . . . . . . . . . . . . . . . . . . . . . . . . 189

C.2 Publications in International Peer-Reviewed Conferences . . . . . . . . . . 190

C.3 Digital Media . . . . . . . . . . . . . . . . . . . . . . . 191

\begin{tabular}{ll}
\hline Bibliography & 193
\end{tabular} 



\section{List of Figures}

$\begin{array}{ll}\text { Chapter 1 } & 1\end{array}$

$1.1 \quad$ Significant milestones in the evolution of Artificial Intelligence. . . . . . . . 2

1.2 Proposed methodology, followed during this thesis. . . . . . . . . . . . . . 5

Chapter 2

$2.1 \quad$ Object detection strategies for addressing scale variation . . . . . . . . . . 12

2.2 Proposed Up-Net architecture designed for electric tower detection and insulator string segmentation . . . . . . . . . . . . . . . . 19

2.3 Tower detection results obtained in several sequences related to inspections of medium voltage power lines $\ldots \ldots \ldots \ldots$. . . . . . . . . 26

2.4 Tower detection results obtained in several sequences related to inspections of high voltage power lines . . . . . . . . . . . . . . . . . . 27

2.5 Examples of images used for training the insulator string segmentation models . . . . . . . . . . . . . . . . . 28

2.6 Insulator string segmentation results obtained by the networks trained without a conditional GAN framework . . . . . . . . . . . . . 33

2.7 Insulator string segmentation results obtained by the networks trained within a conditional GAN framework . . . . . . . . . . . . . . . 34

$2.8 \quad$ Examples of images used for training the helipad detection algorithms . . . 35

2.9 Results of the 5-fold cross-validation procedure for selecting the optimal $\begin{array}{ll}\text { parameters of the supervised learning classifiers used for helipad detection } & 36\end{array}$

2.10 Helipad detection results obtained in several test sequences captured during indoor and outdoor experiments . . . . . . . . . . . . . . . . 38

2.11 UAV altitude estimation using the helipad detection results obtained in the sequences concerning real-flight experiments. . . . . . . . . . . . . . . . 39

Chapter 3

$3.1 \quad$ System description for the diagnosis of electrical insulator strings . . . . . 52

3.2 Proposed Convolutional Neural Network architecture for diagnosing the absence of disc units . . . . . . . . . . . . . . . . . 53

3.3 Proposed Siamese Convolutional Neural Network architecture for diagnosing damaged disc units . . . . . . . . . . . . . . . . . . . 55 
3.4 Proposed Siamese Convolutional Autoencoder architecture for diagnosing damaged disc units . . . . . . . . . . . . . . . 58

3.5 Examples of images used for training the component responsible for diagnosing the absence of disc insulator units . . . . . . . . . . . . . . . 61

$3.6 \quad$ ROC curves generated by the best model for each CNN configuration tested for the disc absence defect diagnosis . . . . . . . . . . . . . . . . . . . 64

3.7 Prediction results obtained by the proposed CNN for diagnosing the absence of disc units . . . . . . . . . . . . . . . . . . . . . 64

3.8 Examples of images used for training the proposed Siamese CNN model . . 65

3.9 Parameter selection for the One-Class SVM model . . . . . . . . . . . . . . 67

3.10 ROC and Precision-Recall curves generated by the best model for each Siamese CNN configuration tested for the diagnosis of damaged disc units. $\quad 70$

3.11 Prediction results obtained by the proposed Siamese CNN for diagnosing

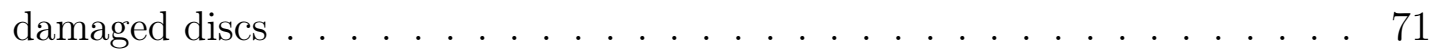

3.12 Prediction results obtained by the damaged disc diagnosis component in four test video sequences corresponding to real aerial inspections of high voltage power transmission lines . . . . . . . . . . . . . . . . 73

3.13 Prediction results obtained by the absent disc diagnosis component in two simulated sequences . . . . . . . . . . . . . . . 75

\section{Chapter 4}

$4.1 \quad$ Architecture of the proposed RL-IBVS system . . . . . . . . . . . . . . . . 85

4.2 Notation and reference frames utilized in the RL-IBVS formulation . . . . 87

4.3 RotorS Gazebo simulation scenarios used for training the RL-IBVS variants 90

4.4 Aerial robotic platforms used for evaluating the IBVS algorithms in real

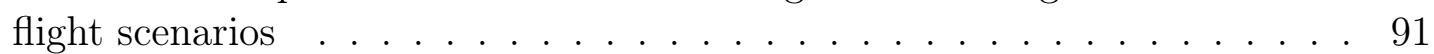

4.5 Training curves obtained during the training process of the 2D RL-IBVS . 92

4.6 Training curves obtained during the training process of the 3D RL-IBVS . 93

4.7 Simulation results obtained for the classic IBVS approach while following a moving target executing a rhomboidal trajectory . . . . . . . . . . . . 95

4.8 Simulation results obtained by the proposed 2D RL-IBVS controller while following a moving target executing a rhomboidal trajectory . . . . . . . 96

4.9 Simulation flight experiment for evaluating the proposed 3D RL-IBVS in a leader-follower configuration, where the leader executes a rhomboidal

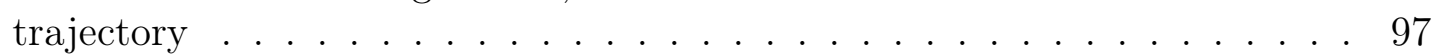

4.10 Simulation flight experiment for evaluating the proposed 3D RL-IBVS in a leader-follower configuration, where the leader executes a sinusoidal trajectory 99

4.11 Scenarios created in real-flight experiments for evaluating the RL-IBVS

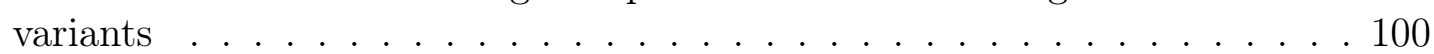

4.12 Real flight experiment for validating the proposed 2D RL-IBVS while performing a rescue mission on a moving target . . . . . . . . . . . . . . . . . 101

4.13 Real flight experiment for validating the proposed 3D RL-IBVS with a human operator as the object to follow . . . . . . . . . . . . . . 103 
4.14 Real flight experiment for validating the proposed 3D RL-IBVS using a remotely controlled car as the object to follow . . . . . . . . . . . . . . . 104

\section{Chapter 5}

$5.1 \quad$ Architecture of the proposed RL-Reactive Navigation system . . . . . . . . 115

5.2 State and repulsive field computation steps . . . . . . . . . . . . . 116

5.3 Gazebo simulation scenario designed for training the RL-Reactive Navigation system . . . . . . . . . . . . . . . . . . 119

5.4 Curves obtained during the training process of the RL-Reactive Navigation

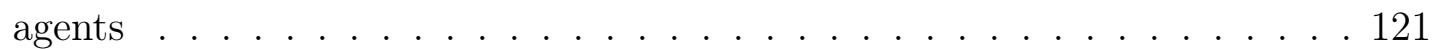

5.5 Gazebo simulation scenarios created for evaluating the performance of the proposed RL-Reactive Navigation agents . . . . . . . . . . . . . . . . . . . 121

5.6 Trajectories generated by the proposed DDPG agents in the simulation flight experiments . . . . . . . . . . . . . . . 123

5.7 Trajectories generated by the proposed NAF agents in the simulation flight experiments . . . . . . . . . . . . . . . . . . . . . 124

5.8 Trajectories generated by the baseline (RRT* + MPC) in the simulation flight experiments . . . . . . . . . . . . . . . . . . 125

5.9 Real Flight experiments designed for evaluating the proposed RL-Reactive Navigation system in long missions with static and dynamic obstacles . . . 127

5.10 Real flight experiment designed for evaluating the proposed RL-Reactive Navigation system using a fast-moving obstacle . . . . . . . . . . . . . . . 128

\section{Chapter 6}

$6.1 \quad$ Aerostack architecture showing the proposed mission planning system . . . 138

6.2 Flowchart of the proposed mission planning system . . . . . . . . . . . . 142

6.3 State Pattern implemented in the Manager of Actions . . . . . . . . . . . . 145

6.4 Frames of reference defined for the execution of autonomous SAR missions 149

6.5 Simulation scenarios designed using ROS Rviz for evaluating the proposed mission planning system while managing swarms of UAVs . . . . . . . . . . 153

6.6 Results obtained in the evaluation of the proposed mission planning system while managing swarms of UAVs for executing high-level missions . . . . . 155

6.7 Real flight experiment using two UAVs for a find target mission . . . . . . 156

6.8 Proposed aerial robot used for SAR missions . . . . . . . . . . . . . . . 158

6.9 Results obtained in the evaluation of the proposed system during the execution of different SAR missions in simulation flight experiments . . . . . . 160

6.10 Real-flight scenarios designed for evaluating the proposed system in SAR missions. . . . . . . . . . . . . . . . . . 161

6.11 Results obtained in the evaluation of the proposed system during the execution of different SAR missions in real-flight experiments . . . . . . . . . 163 
\begin{tabular}{ll}
\hline Appendices & 169
\end{tabular}

A.1 Usual neural network activation functions . . . . . . . . . . . . . . . . 173

A.2 Exponentially Weighted Moving Average intuition . . . . . . . . . . . . . . 175

B.1 Examples of images used for training the supervised learning classifiers for bucket/background classification . . . . . . . . . . . . . . . 182

B.2 Results obtained in the comparison of the supervised learning classifiers used for bucket/background classification on 6 different evaluation tests . . 184

B.3 Average $F_{1}$ score and processing time for the different supervised learning classifiers from the results presented in Table|B.3 . . . . . . . . . . . . . 186

B.4 Object detection results for target 1 obtained during a real-flight SAR mission 187

B.5 Object detection results for target 2 obtained during a real-flight SAR mission 188 


\section{List of Tables}

Chapter 2

$2.1 \quad$ Results obtained in the evaluation of the proposed FCN and baseline methods for electric tower detection in two real aerial inspections of medium voltage power lines . . . . . . . . . . . . . . . . . 24

2.2 Results obtained in the evaluation of the proposed FCN and baseline methods for electric tower detection in two real aerial inspections of high voltage

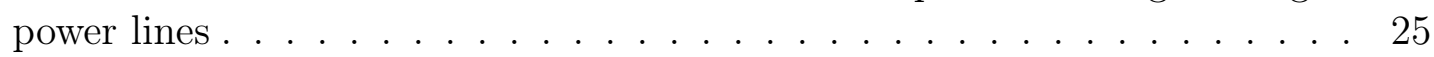

2.3 Insulator string segmentation results on the test set composed of 240 images 32

2.4 Results obtained in the evaluation of the proposed method for helipad

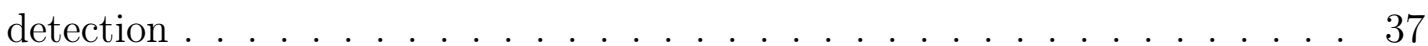

Chapter 3

3.1 CNN architectures designed for diagnosing the absence of disc insulator units 54

3.2 CNN architectures inside each SCNN configuration, designed for diagnosing damaged disc units .................... 56

3.3 CNN architectures designed for each SCAE configuration, used for diagnosing damaged disc units . . . . . . . . . . . . . . . . . . . . . 60

3.4 Results obtained for diagnosing the absence of disc insulator units on a test set of 400 images . . . . . . . . . . . . . . . . 63

3.5 Results obtained for the diagnosis of damaged disc units on a test set

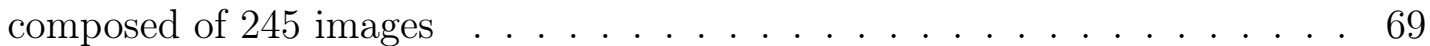

3.6 Comparison between the supervised and semi-supervised learning frameworks for diagnosing damaged discs . . . . . . . . . . . . . . . 70

$3.7 \quad$ Results obtained in the evaluation of the proposed diagnosis system using five video sequences of real aerial inspections . . . . . . . . . . . . . . 72

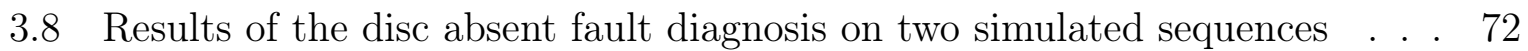

\section{Chapter 4}

4.1 Average errors measured in pixels and percentage overshoot obtained for the simulation flight experiment of Figures 4.7 and $[4.8$. . . . . . . . . . . . 95

4.2 Average errors measured in pixels and percentage overshoot obtained for the simulation flight experiment of Fig. 4.9 ] . . . . . . . . . . . . . . . . . 98 
4.3 Mean and standard deviation errors in pixels obtained for the real flight experiment of Fig. 4.13 . . . . . . . . . . . . . . . . . . 102

Chapter 5

5.1 Configurations analyzed for the DDPG (actor and critic) and NAF (Qnetwork) agents. . . . . . . . . . . . . . . . . 120

5.2 Evaluation of the different reactive navigation agents in the simulation scenarios of Fig. $5.5 \ldots \ldots \ldots \ldots$. . . . . . . . . . . . . . . . . . . . . . . .

Chapter 6

133

6.1 Example of the initial state of the objects in a SAR mission . . . . . . . . 148

6.2 Experiment set designed for real and simulation flights using swarms of UAVs 152

\section{Appendices}

B.1 CNN configurations evaluated for bucket/background classification. . . . . 183

B.2 Average training results obtained for the 6 evaluation tests of Fig. B.2a| Final selected model is indicated in bold. . . . . . . . . . . . . . . . . . . . 184

B.3 Average test results obtained for the 6 evaluation tests of Fig. $\mid$ B.2b. Final selected model is indicated in bold. . . . . . . . . . . . . . . . . . . 185 


\section{Chapter 1}

\section{Introduction}

The advances in computational resources have permitted a rapid growth in the development of new machine learning algorithms, especially regarding the design of deep learning algorithms. Deep neural networks were relegated in the $90 \mathrm{~s}$ by more efficient learning-based algorithms such as Support Vector Machines (SVMs) Boser et al., 1992, Vapnik, 1999. The development of new hardware components during the last 15 years, such as GPUs, together with a massive acquisition and treatment of data, allowed the application of more sophisticated but computationally expensive neural networks such as Convolutional Neural Networks (CNNs). Nowadays, the available computational resources allow training very deep neural networks, composed of millions of parameters, in just a few hours. Furthermore, once these networks are trained, the inference is also performed relatively fast in most of the current computers which incorporate in most of the cases powerful CPUs and GPUs.

\subsection{Learning-based algorithms for robotics}

In the robotics field, learning-based algorithms for perception, navigation, control, and planning are gaining significant importance due to their versatility. The state-of-theart algorithms in these fields have been designed on the basis of consolidated Artificial Intelligence $(\mathrm{AI})$ algorithms, which suffered a tremendous evolution during the last 70 years (see Fig. 1.1 $]^{2}$. Regarding the perception task, most of the requirements imposed on actual robotic platforms and autonomous vehicles involve the high-level understanding of their environment. This is translated into high-level capabilities for object detection and classification, which can greatly help the decision-making abilities of the robotic platforms towards the development of fully-autonomous robotic agents. Most of the current state-the-of-art algorithms developed for object detection and classification are based on well-established methods developed during the last 70 years. The Neocognitron proposed in Fukushima, 1980 was one of the precursors of todays modern CNNs. Subsequently, the development of the backpropagation algorithm (Rumelhart et al., 1986) was one of the most important milestones of the last decades, since it allowed the efficient computation

\footnotetext{
${ }_{1}$ adapted from: https://journal.jp.fujitsu.com/en/2016/02/09/01/
} 
of the gradients used in the gradient descent optimization method (among others) to train neural networks. Even today is one of the fundamental algorithms used for training neural networks. Despite CNNs were proven to provide outstanding results in digit recognition back in the 90s (LeCun et al., 1998; LeCun et al., 1999), it was in 2012 with the development of the well-known AlexNet architecture (Krizhevsky et al., 2012) when the massive use of CNNs started. The performance of these and subsequent models showed the power of machine learning techniques (in this specific case, deep supervised learning techniques) for solving complex computer vision problems, such as object detection and classification, object tracking, gesture recognition, etc. Nowadays, research efforts are aligned towards the development of deeper, and more complex versions of the CNNs, which are still the basic building block in most of the state-of-the-art object detection and classification algorithms.

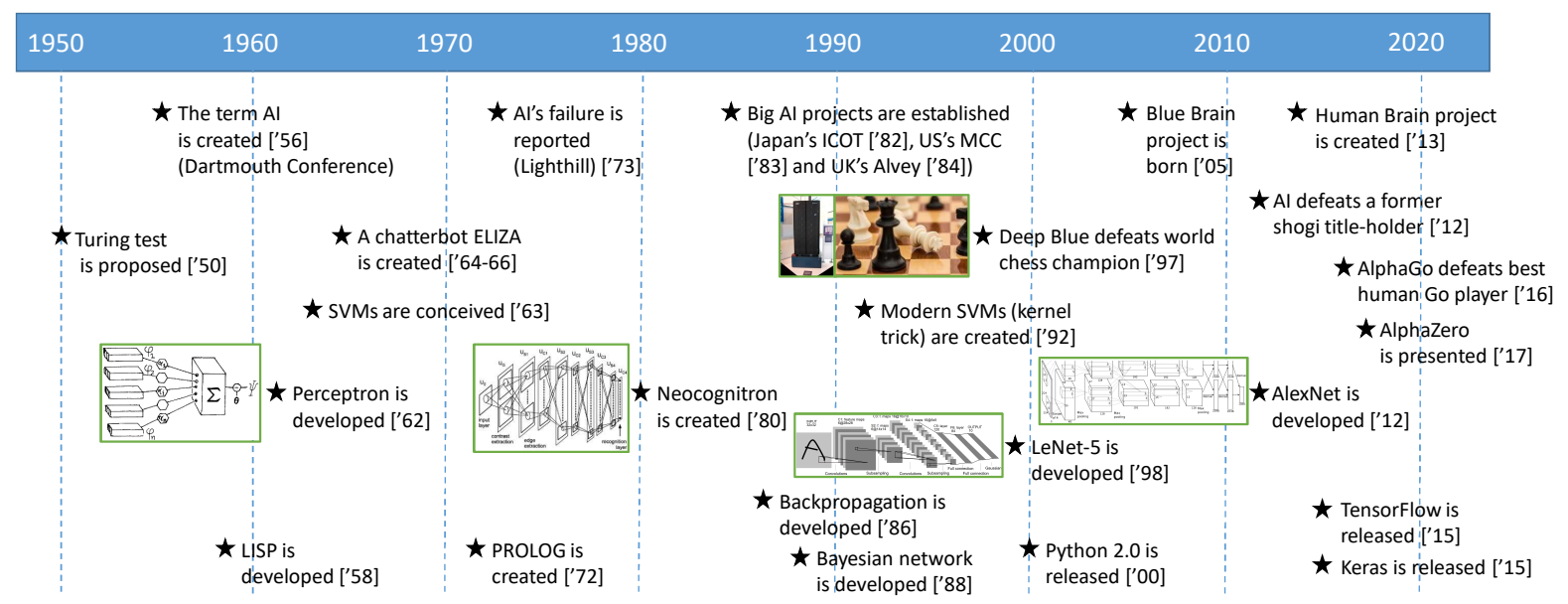

Figure 1.1: Significant milestones in the evolution of Artificial Intelligence.

Regarding the navigation, control, and planning tasks, although a wide variety of learning-based algorithms have been proposed in the literature, we would like to highlight the important growth of deep reinforcement learning (DRL) algorithms. Most of these algorithms mimic the learning process of living beings trying to learn from experience, giving a sequence of rewards in order to guide the learning process. Modern DRL algorithms (Mnih et al., 2015; Lillicrap et al., 2015; Silver et al., 2017; Silver et al., 2018) revealed the outstanding results obtained by computers learning from experience. In these works, DRL algorithms were able to provide feasible solutions to complex games and even defeat human champions in their respective fields. Furthermore, modern DRL algorithms integrate neural networks in order to evaluate the learning process or even learn near-tooptimal policies. Besides, the rapid evolution of more realistic robotic simulators allows training this kind of algorithms for a wide variety of robotic tasks. Based on all the aforementioned characteristics, current research in learning-based robotics is focused on the development of faster and easy-to-use DRL algorithms for learning complex behaviors. 


\subsection{Motivation of the Thesis}

The works presented in this thesis have been constantly motivated by the continuous progress and achievements in the field of AI during the last years, such as the ones presented in the previous section involving the use of supervised learning and deep reinforcement learning procedures. However, most of these high-impact works, are based on the assumption that powerful machines are always available. As an example, in most of the challenges in the field of AI, only the performance is taken into account, and little attention is put in the feasibility of integration of these powerful models into platforms with certain computational constraints such as mobile robotic platforms. These computational constraints are even more severe in the field of aerial robotics, where usually the small size of the aerial robot forces the use of smaller computers, and therefore, with less computational capabilities. Furthermore, aerial robots are designed for operating in a wide range of conditions, such as unstructured outdoor and indoor scenarios. In such situations, a wide range of events and possible failures can appear during the execution of the current mission. For this reason, the algorithms developed for being integrated into aerial robotic platforms (e.g., for object detection, control, navigation, planning, etc.), apart from showing real-time capabilities, must be robust for being adapted to the aforementioned situations. This analysis has motivated most of the work presented in this thesis, where special attention has been conducted in the development of algorithms that can be integrated to operate into an actual aerial robotic platform.

Furthermore, one of the most important motivations for the works presented in this thesis is the thorough analysis of the limits of learning-based algorithms applied in the aerial robotics field, and the feasibility of learning complex behaviors as living beings do (i.e., learning from observation and experience). Based on the outstanding results of leaning-based and biologically-inspired algorithms, several questions emerge related to the feasibility of their application for solving the wide range of tasks that a robotic agent is designed to achieve.

Added to the above motivations, is the final goal that has motivated scientist and researchers in the robotics field during decades, which is the development of a fullyautonomous agent. That is, a robotic agent able to perform complex missions (e.g., exploration and surveillance, search and rescue, etc.) without human intervention. This goal requires the development of flexible algorithms capable of being adapted to operate in conjunction with other algorithms of varying nature. Besides, the final integration of all the algorithms into components of a real robotic architecture in order to provide reliable and safe capabilities, requires thorough evaluation and experimentation, not only for the components working in isolation but in conjunction with the whole system. This motivation adds a higher level of complexity to the requirements previously established for the development of algorithms integrated into a mobile robotic platform. 


\subsection{Objectives of the Thesis}

Based on the previous motivation, the following objectives were defined:

O.1 The main objective of this thesis is defined to provide a solution to one of the main motivations explained in Section 1.2, which involves the development of a fullyautonomous aerial robotic agent, capable of performing high-level missions without human intervention. An extension of this objective is aimed at the development of a fully-autonomous aerial robot using learning-based techniques. That is, running learning-based algorithms in most of the components of the robotic architecture. To achieve this high-level objective, other intermediate objectives have been defined, and are summarized next.

O.2 The achievement of the previous objective, with the appropriate capabilities for performing high-level missions, requires versatile planning components with the appropriate flexibility to manage very different components which should operate in a wide range of missions. Thus, the second objective is aimed towards the development of a Mission Planning system able to manage a fully-autonomous aerial robotic agent. Furthermore, an extension of this objective is aimed at providing a flexible mission planning system capable of managing a multi-robot system (e.g., a swarm of aerial robots).

O.3 The third objective is focused on the visual perception components in a robotic architecture. The analysis of state-fo-the-art architectures for designing fully-autonomous robotic agents allows starting thinking and analyzing the process of how these robotic agents are going to perceive and understand their environment. This question is of particular interest in some missions, such as SAR, where the aerial robot should interact with a target to conduct the rescue operation. For this purpose, the current objective is defined towards the design and development of reliable object detection algorithms which must be flexible enough to operate under a wide range of conditions, robust to ensure precision in the accomplishment of the corresponding mission, and fast for running onboard an aerial robotic platform.

O.4 The accomplishment of fully-autonomous missions, such as SAR, requires the development of the appropriate components for ensuring safe navigation (i.e., with obstacle avoidance capabilities) within the environment where the mission is executed, and the interaction with the objects of interest. This objective is joined with the motivation of adapting the learning process of living beings to the learning process of a robotic agent. That is, to analyze the feasibility of learning complex behaviors (e.g., reactive navigation, object following) directly from experience (i.e., as in the reinforcement learning paradigm). For this purpose, the definition of this objective is aimed at designing and developing learning-based algorithms to provide the appropriate capabilities to navigate in indoor environments safely, and to interact with the objects of interest located within the environment (e.g., targets in a SAR mission). 


\subsection{Methodology}

The methodology followed during the development of this thesis, shown in Fig. 1.2, is based on the combination of the SIMILAR process proposed in Bahill and Gissing, 1998, and a soft version of the V-model (Forsberg and Mooz, 1991).

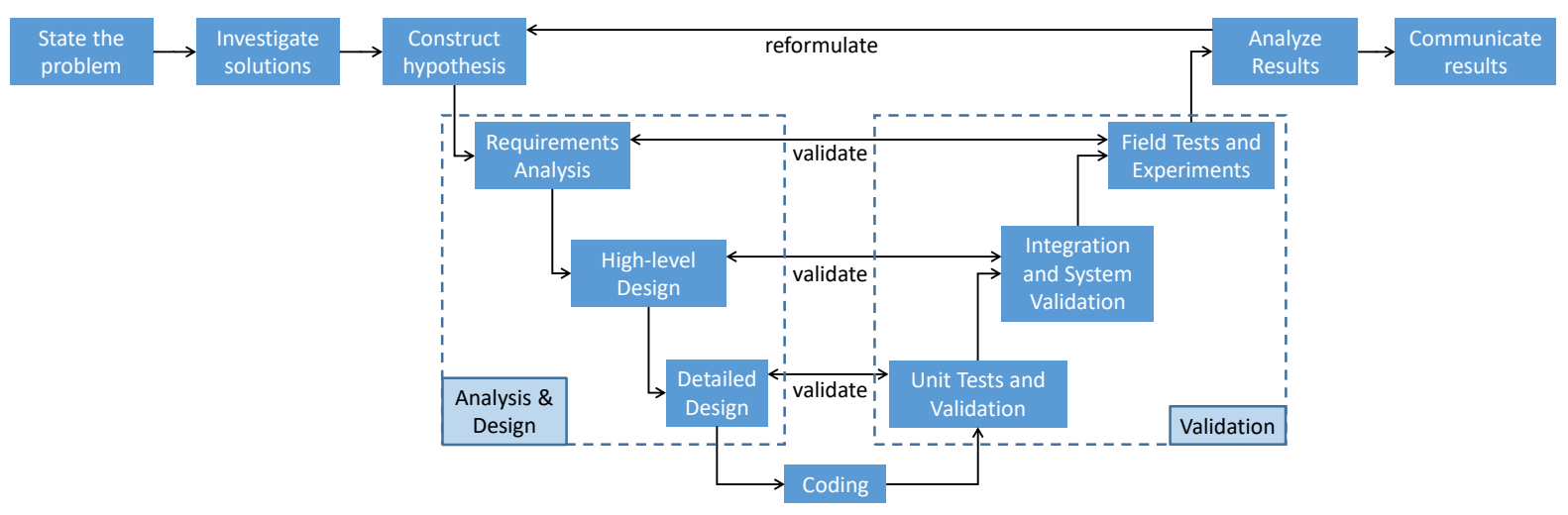

Figure 1.2: Proposed methodology, followed during this thesis.

The proposed methodology can be summarized in the following steps:

- Problem statement and hypothesis formulation: This step involves the process from the identification of a specific problem, to the hypothesis formulation in order to provide feasible solutions to the problem. In this process, the first step consists of identifying the problem that needs to be solved and characterize it. Usually, a good problem statement is defined by previously formulating the appropriate questions. Once the problem has been characterized, the subsequent step requires to perform research in order to investigate state-of-the-art methods that address in a partial or complete way the problem defined previously. The final step involves the formulation of the hypothesis conceived to solve the problem, which requires a first stage for the identification of the objectives.

- Analysis and Design: This process involves first the collection of the requirements based on the objectives defined in the previous step, in order to clearly establish what the proposed system has to perform. This complete process is inherited from the software engineering process, from which we adapt the user-defined requirements to the research community requirements. That is, based on the previous research conducted on state-of-the-art solutions, we formulate the appropriate questions to design the requirements in order to contribute to the body of knowledge. Subsequently, the system and architecture design is conducted. In this step, the system is first conceived by identifying the techniques to implement the requirements defined previously. Based on the possible implementations, the next step consists of the software architecture design, followed by a low-level design of the components (e.g., object detection, reactive navigation, visual control, etc.). In this step, the communications (e.g., inputs ad outputs) with other components of the architecture 
are defined, together with a proper definition of the functionalities of each module within the component. This definition benefits the next stages for the actual coding of the proposed implementation. In the specific case of this thesis, the Analysis and Design process is of crucial importance since most of the components developed to achieve the objectives described in Section 1.3, are integrated into a complete architecture for aerial robotics.

- Experimentation and validation: This process involves a sequence of validation steps. First, unit tests are conducted to analyze the performance of each component separately and eliminate possible errors before its integration and validation with the whole system. After unit tests are passed, the integration of the different components is performed following a sequential procedure. Subsequently, we proceed with the system validation to ensure that the requirements defined in the first stages are appropriately covered and the final objectives can be achieved. In this stage, aerial robotic simulators acquire an utmost importance since they provide a complete framework to conduct proper validation of the proposed system before conducting final field tests and experiments. Finally, after the validation of the proposed system, usually conducted throughout this thesis using aerial robotics simulators, the integration of the proposed system onboard the aerial robotic platform is conducted. This requires a previous design stage for the experiments that are required to be conducted in real-flight conditions. If some errors or other possible limitations of the system are identified, new requirements can be defined to perform a new iteration in the process.

- Analysis of the results: Based on the results obtained after the previous step (i.e., usually gathering all the information related to real but also simulation tests), a thorough analysis of the data is performed. This stage usually involves the creation of graphs and tables in order to facilitate the visualization and interpretation of the results. After the analysis of the results, conclusions are extracted. If some findings suggest that an additional or better hypothesis might be formulated, another iteration of the whole process can be started. On the other hand, if the findings and conclusions are considered to contribute to the body of knowledge, the subsequent stage for scientific dissemination is started.

- Scientific dissemination: This step consists of communicating the results to the scientific community, which usually involves writing scientific documents to elaborate the formulation of the hypothesis, present the system conceived to its implementation, and present the experiments and results for the comprehension of the study.

\subsection{Contributions and Dissertation Outline}

Several contributions have been achieved in this thesis within the field of supervised and semi-supervised learning $\mathbf{C . 1}$ and $\mathbf{C . 2}$, deep reinforcement learning $(\mathbf{C . 3}$ and $\mathbf{C . 4}$, and mission planning (C.5. In fulfillment of the objectives presented in Section 1.3, the following contributions have been achieved: 
C.1 Several object detection and segmentation algorithms in RGB images based on classical machine learning methods, and more sophisticated Fully Convolutional Networks (FCNs) are presented in Chapter 2, More concretely, we propose a novel FCN which is successfully applied to solve the use case of electric tower detection and insulator string segmentation as a prior stage for fault diagnosis. A thorough evaluation of the proposed FCN architecture is conducted by comparing its performance to consolidated state-of-the-art object detectors and semantic segmentation methods, revealing its flexible design and outstanding performance in a wide range of conditions. Furthermore, we also propose a fast method for solving the helipad detection use case as a prior stage for autonomous landing missions. The proposed method for helipad detection is designed for optimizing its performance in computers with standard CPUs, using lightweight algorithms. The proposed object detection and segmentation algorithms provide a versatile solution to objective $\mathbf{O . 3}$.

C.2 Based on the previous contribution, Chapter 3 introduces novel algorithms conceived for anomaly detection in RGB images applied to the use case of electric power transmission line inspection and diagnosis. For this purpose, we first present a novel Siamese Convolutional Neural Network (SCNN) designed for modeling the dissimilarity between consecutive elements within the insulator strings. The proposed SCNN is mainly trained using synthetic data and is capable of achieving outstanding diagnosis results in several video sequences of real aerial inspections. We further propose a novel Siamese Convolutional Autoencoder (SCAE) which is obtained by a two-stage training, involving the training of a CAE and its subsequent adaptation into a siamese architecture, which is finally joined with a One-Class SVM classifier. The latter architecture is only trained using normal samples (i.e., assuming that only healthy examples are available) and is able to perform remarkably well in the diagnosis of normal and anomalous samples at test time.

C.3 After providing versatile solutions for the visual perception tasks, a robotic agent is often required to interact with the detected target, as humans do. In this thesis, we conceive the interaction process as a matter of following the detected target or responding to some requested events from the current mission (e.g., rescue operations in SAR missions). For this purpose, Chapter 4 describes a novel Image-Based Visual Servoing through Reinforcement Learning (RL-IBVS) algorithm which is completely trained in simulation and evaluated in several simulation and real flight experiments. To this aim, a complete RL framework for training aerial robotic agents to learn IBVS policies is proposed. Furthermore, we propose two variants of the main RLIBVS algorithm for their operation in 2D (2D RL-IBVS) and 3D (3D RL-IBVS) spaces. An extensive evaluation of the proposed RL-IBVS methods has been conducted by comparing their performance to traditional IBVS techniques, showing outstanding IBVS capabilities in simulation and real flight experiments. The proposed RL-IBVS algorithms provide a partial fulfillment of the objective $\mathbf{O . 4}$.

C.4 In order to provide a complete solution to objective $\mathbf{0 . 4}$, in Chapter 5 we present a novel target-driven reactive navigation algorithm based on deep reinforcement 
learning techniques, which is only trained in simulation and evaluated in simulation and real flight conditions. The proposed algorithm is trained using the reinforcement learning framework proposed in C.3, which is adapted in this case for reactive navigation purposes. The proposed algorithm introduces a novel formulation for defining the reward function of the agent, based on Artificial Potential Fields (APFs), which allowed to accelerate the training process of the agent while providing good generalization capabilities for reactive navigation purposes. Furthermore, we present an extensive validation of the proposed algorithm in simulation and real flight experiments involving static and dynamic obstacles.

C.5 This contribution aims at providing a solution to objective $\mathbf{0 . 1}$ and $\mathbf{0 . 2}$. For this purpose, Chapter 6 presents a versatile and flexible mission planning system, based on the combination of a Global Mission Planner (GMP) and an Agent Mission Planner (AMP), which allow the management of fully-autonomous missions using a single or a multi-robot configuration. The proposed GMP is designed in a userfriendly way and allows the definition of complex missions (e.g., find target, SAR, etc.) task by task using an XML-based language or automatically generating the whole mission by providing only a high-level command. In addition, the proposed GMP allows real-time mission re-planning in response to several events that can occur during the execution of the mission (e.g., target found in SAR missions). For this purpose, the GMP implements an active list of missions which allows real-time mission concatenation and re-planning. On the other hand, the proposed AMP acts at the agent level of the architecture, and is in charge of scheduling task by task the proposed mission, providing at the same time important capabilities such as dynamic mission point reallocation. A thorough validation of the proposed mission planning system is conducted in simulation and real flight experiments, using swarms of aerial robots and testing its capabilities in complex SAR scenarios.

C.6 As an additional contribution, the author would like to highlight the extensive experimentation that has been performed during the whole thesis. A wide range of deep learning techniques involving the application of data augmentation, transfer learning, adversarial training using Generative Adversarial Networks (GANs), among other techniques, have been analyzed and developed throughout Chapters 2 and 3. Furthermore, extensive simulation and real-flight experiments in a wide range of scenarios have been conducted throughout Chapters 4 , 5 , and 6 .

Finally, we provide the conclusions of the entire dissertation in Chapter 7 , highlighting the future research directions. Additional material is provided in Appendix $\mathrm{A}$, where some important background in deep learning and deep reinforcement learning is provided for a complete understanding of the methods applied in this thesis. Furthermore, we provide additional object detection results in Appendix B, obtained during the experiments concerning SAR missions, and finally, Appendix C provides a complete list of publications in which the author of this thesis has actively participated during the research period in the CVAR group. 


\subsection{List of Publications}

The work presented in this Thesis is based on the following publications:

\subsubsection{Journal Publications}

- Carrio, A., Sampedro, C., Rodriguez-Ramos, A., \& Campoy, P. (2017). A review of deep learning methods and applications for unmanned aerial vehicles. Journal of Sensors, 2017. JCR 2018: 2.024 (All chapters)

- Sampedro, C., Rodriguez-Ramos, A., Bavle, H., Carrio, A., de la Puente, P., \& Campoy, P. (2018). A fully-autonomous aerial robot for search and rescue applications in indoor environments using learning-based techniques. Journal of Intelligent \& Robotic Systems, 1-27. JCR 2018: 2.020 (Chapters 4 and 6)

- Sampedro, C., Rodriguez-Vazquez, J., Rodriguez-Ramos, A., Carrio, A., \& Campoy, P. (2019). Deep Learning-Based System for Automatic Recognition and Diagnosis of Electrical Insulator Strings. IEEE Access, 7, 101283-101308. JCR 2018: 4.098 (Chapters 2 and 3)

\subsubsection{Publications in International Peer-Reviewed Conferences}

- Sampedro, C., Bavle, H., Sanchez-Lopez, J. L., Fernandez, R. A. S., RodriguezRamos, A., Molina, M., \& Campoy, P. (2016, June). A flexible and dynamic mission planning architecture for uav swarm coordination. In 2016 International Conference on Unmanned Aircraft Systems (ICUAS) (pp. 355-363). IEEE. (Chapter 6)

- Sampedro, C., Bavle, H., Rodriguez-Ramos, A., Carrio, A., Fernandez, R. A. S., Sanchez-Lopez, J. L., \& Campoy, P. (2017, June). A fully-autonomous aerial robotic solution for the 2016 international micro air vehicle competition. In 2017 International conference on unmanned aircraft systems (ICUAS) (pp. 989-998). IEEE. (Chapter 6)

- Rodriguez-Ramos, A., Sampedro, C., Bavle, H., Milosevic, Z., Garcia-Vaquero, A., \& Campoy, P. (2017, June). Towards fully autonomous landing on moving platforms for rotary unmanned aerial vehicles. In 2017 International Conference on Unmanned Aircraft Systems (ICUAS) (pp. 170-178). IEEE. (Chapter 2)

- Sampedro, C., Rodriguez-Ramos, A., Gil, I., Mejias, L., \& Campoy, P. (2018, October). Image-Based Visual Servoing Controller for Multirotor Aerial Robots Using Deep Reinforcement Learning. In 2018 IEEE/RSJ International Conference on Intelligent Robots and Systems (IROS) (pp. 979-986). IEEE. (Chapter 4)

- Sampedro, C., Bavle, H., Rodriguez-Ramos, A., de la Puente, P., \& Campoy, P. (2018, October). Laser-Based Reactive Navigation for Multirotor Aerial Robots using Deep Reinforcement Learning. In 2018 IEEE/RSJ International Conference on Intelligent Robots and Systems (IROS) (pp. 1024-1031). IEEE. (Chapter 5 ) 
- Lu, L., Sampedro, C., Rodriguez-Vazquez, J., \& Campoy, P. (2019, June). Laserbased Collision Avoidance and Reactive Navigation using RRT* and Signed Distance Field for Multirotor UAVs. In 2019 International Conference on Unmanned Aircraft Systems (ICUAS) (pp. 1209-1217). IEEE. (Chapter 5)

The rest of the publications that have contributed indirectly to the achievement of this $\mathrm{PhD}$ thesis or in which the author of this thesis has actively participated are shown in Appendix C. 


\section{Chapter}

\section{Object Detection and Segmentation}

\subsection{Introduction}

Perception and understanding of the environment in which the robotic platforms operate have concentrated much of the efforts in the robotics research community during the last years. Inside the field of robotic perception, object detection is one of the most addressed problems in the literature owing to its implications in the subsequent stages for navigation, localization and mapping, and planning of the robotic platform. Detection of the objects of interest within the images captured onboard the robotic platforms requires the development of versatile and robust algorithms capable of dealing with several problems such as vibrations or sudden movements of the robotic platform, heterogeneous backgrounds, changes in illumination, etc.

In order to achieve robust detection capabilities under the constraints mentioned above, in recent years classical computer vision techniques are being substituted by more versatile deep learning solutions such as Convolutional Neural Networks (CNNs), which act as the core of most of the state-of-the-art algorithms for object detection. Strategies based on traditional computer vision algorithms can provide appropriate results in images acquired under controlled illumination and background conditions. However, current robotic applications are designed for their operation in previously unseen scenarios, both indoors and outdoors, where the robotic platform is usually required to interact with its environment. In such conditions, classical computer vision approaches need several parameters and thresholds which need to be manually tuned and re-adjusted to properly operate under previously unseen conditions. In contrast, strategies based on machine learning techniques, when trained in meaningful datasets, can provide more flexible solutions, making the object detection tasks more robust to changes in illumination, background, type of object, etc. As a drawback, machine learning models and especially deep learning ones, usually require a large amount of data to be trained appropriately.

Regarding the object detection in images, research efforts have been focused on three main aspects: generating region proposals, the feature extraction process, and the problem of scale variation. Generating region proposals refers to the process of creating Regions Of Interest (ROIs) within the image which have a high probability of containing objects of interest, independently of their class. In the past decade, there exists a clear evolution 
from approaches based on classical image processing techniques (e.g., Sliding Window or the Selective Search method proposed in Uijlings et al., 2013) to more innovative deep learning solutions (e.g., Region Proposal Network (RPN) introduced in Ren et al., 2015). Similarly, the feature extraction process has evolved from hand-engineered feature extraction methods such as the widely used Histogram of Oriented Gradients (HOG) introduced in Dalal and Triggs, 2005, to more advanced solutions involving the use of deep convolutional networks such as CNNs which provide unsupervised feature extraction capabilities. Concerning the scale variation problem, it has been considered in the literature as the most challenging problem in object detection tasks. This problem is crucial in object detection systems since one of the most important requirements in such systems is to provide accurate detection of the objects independently of their size in the image. In this direction, several strategies have been proposed (see Fig. 2.1) ranging from multi-scale image pyramids (see Fig. 2.1a) to more sophisticated feature pyramids (see Fig. 2.1d and 2.1e). The latter methods involving feature pyramids are based on the combination of high-resolution semantically poor feature maps with low-resolution semantically rich feature maps, which is usually achieved by adding skip connections to the network. As a result, a network with rich semantics at all scales of the pyramid can be obtained.

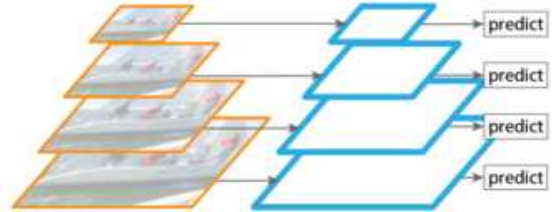

(a)

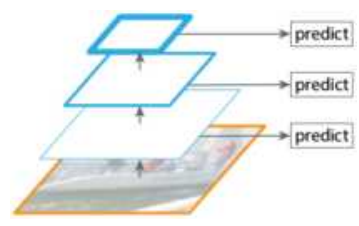

(c)

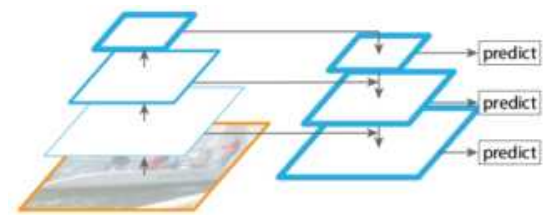

(d)

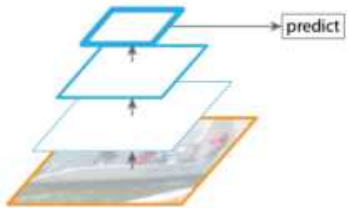

(b)

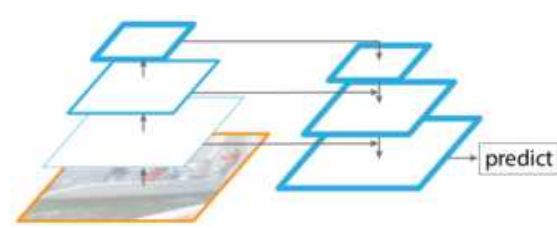

(e)

Figure 2.1: This figure is adapted from Lin et al., 2017, to illustrate different scale variation strategies. (a) Building a feature image pyramid from an image pyramid. (b) Pyramidal feature hierarchy and prediction in a single scale. (c) Pyramidal feature hierarchy and prediction at multiple scales. (d) Feature Pyramid Network (FPN) (Lin et al., 2017). (e) FPN with prediction at a single scale.

In this chapter, we explore different techniques for object detection and segmentation in RGB images using learning-based algorithms, which are applied for solving perception tasks in real-world industry applications. Two primary use cases have been analyzed:

- Autonomous landing on mobile platforms: This use case is motivated by the requirement for performing precise landing maneuvers using multirotor aerial robots on top of platforms which can exhibit sudden movements. The algorithms developed within this use case need to be computationally efficient since they should 
operate onboard the robotic platform and need to be robust to sudden changes in the movement of the aerial robot. To this problem, it is added the vibrations of the camera, which can hamper the detection of the landing site. In this case, we adopt the helipad as the object to be detected since it is commonly utilized in the literature for landing purposes. To address all the constraints mentioned above, we propose a fast object detection algorithm based on a shape-based region proposal method which is combined with a HOG feature extractor and a Support Vector Machine (SVM) classifier, featuring real-time capabilities on a regular CPU.

- Automatic inspection of electrical power transmission lines: The objective in this use case is to detect the different elements that compose the power transmission lines infrastructure (e.g., electric towers and insulators) to apply subsequent stages for fault diagnosis. To this aim, a novel Fully Convolutional Network (FCN) similar to the one shown in Fig. 2.1e is proposed, which has proven to perform properly in several object detection and segmentation tasks in overhead power lines.

In this chapter, we divide the learning-based algorithms developed for object detection into two subcategories. The algorithms which compute object proposals before the application of a supervised learning algorithm for object classification are explained in Section 2.3. Subsequently, the algorithms based on Fully Convolutional Networks (FCNs) are described in detail in Section 2.4.

\subsection{Related Work}

Regarding the process of generating region proposals, the state-of-the-art algorithms can be divided into two general categories: one-stage and two-stage methods. The latter methods refer to the approaches that use a separate region proposal algorithm before the application of a supervised learning classifier. In the last decade, two-stage methods have evolved from using standard image processing techniques to the use of CNNs for creating those proposals. Several strategies have been proposed in the literature for addressing the region proposal process using classic image processing techniques, such as Selective Search (Uijlings et al., 2013), methods based on objectness (Alexe et al., 2010, Cheng et al., 2014) or Edge Boxes (Zitnick and Dollár, 2014). Sliding Window techniques were also very common in object detection strategies (Dalal and Triggs, 2005). One of the most representative two-stage methods which resulted in the appearance of an entire family of object detectors, is the R-CNN proposed in Girshick et al., 2014. In R-CNN, object proposals are generated using the Selective Search approach, after which a CNN was used to extract the features of each proposal independently, and finally an SVM with linear kernel was used to classify them. The inherent computational cost of R-CNN was further addressed by methods such as SPPnet (He et al., 2015b), where a Spatial Pyramid Pooling (SPP) layer was introduced to generate a fixed-length representation regardless of the input image size. Beyond, Fast R-CNN proposed in Girshick, 2015 was conceived for addressing the problems presented in R-CNN and SPPnet. In Fast R-CNN, an ROI pooling layer was designed to operate over each ROI proposal to generate a fixedlength vector, which is subsequently processed by a sequence of fully-connected layers that 
finally branch into two sibling output layers. One of the branches is used for classifying the input ROI into one of the corresponding pre-defined classes, and the other output branch is used as a bounding box regressor for refining the detection. Built on top of Fast R-CNN, Faster R-CNN (Ren et al., 2015) used the concept of anchors to create a Region Proposal Network (RPN) which consists of an FCN used for generating object proposals which are then processed by Faster R-CNN. As compared to two-stage object detectors, one-stage methods aim at providing object detection and classification results using a single stage, that is, omitting the proposal generation stage. This kind of object detectors were popularized by YOLOv2 (Redmon and Farhadi, 2016), YOLOv3 (Redmon and Farhadi, 2018), and SSD (Liu et al., 2016b), where the concept of anchor is again used for refining the bounding boxes coordinates predicted inside an FCN.

With respect to the feature extraction process, hand-engineering methods have evolved towards unsupervised feature extraction using convolutional networks. Several handcrafted feature extractors such as LBP (Ojala et al., 1996), SIFT (Lowe, 2004), or HOG (Dalal and Triggs, 2005) have been widely applied in the field of object detection and classification. HOG was the de facto feature extractor in Deformable Part-based Models (DPM) (Felzenszwalb et al., 2009, Azizpour and Laptev, 2012) which were consolidated as the most powerful object detection algorithms before the arrival of CNNs. One of the first works showing the reliable capabilities of CNNs for object classification was the AlexNet architecture proposed in Krizhevsky et al., 2012, which consists of a CNN with 5 convolutional, 3 max-pooling, and 3 fully-connected layers, obtaining the best classification results in the 2012 ImageNet competition. In the same direction, OverFeat (Sermanet et al., 2013) showed how CNNs can be used as a sliding window detector on an image pyramid. To this work followed the aforementioned R-CNN family of detectors which consolidated the use of CNNs as feature extractors.

With the problem of generating region proposals and the process of feature extraction being in an advanced development state, the problem of scale variation remains as one of the most challenging issues in object detection. To this regard, methods based on image pyramids (Adelson et al., 1984) (see Fig.2.1a) were largely used in the era of hand-engineered features. However, these methods are computationally costly since inference to the network is performed for each level of the pyramid, making this approach impractical for real-time applications. In addition, the training process requires a considerable amount of memory resources, which reduces the utility of this type of approaches. Conversely, methods based on feature pyramids (He et al., 2015b; Girshick, 2015, Ren et al., 2015) can be more efficient in terms of computational cost since one forward pass to the network is sufficient to compute all the features at different levels of the pyramid. A more efficient solution, leveraging the inherent in-network feature pyramid created by a CNN, was proposed in SSD (see Fig.2.1c), where the predictions for object detection were performed using the feature maps at each level of the CNN. Most recent approaches in object detection and semantic segmentation models have tried to incorporate the strong semantic information of deeper levels of the CNN-based architecture with the low-level features extracted by shallower levels. For this purpose, "skip" connections (Bishop, 2006) were introduced in Long et al., 2015 in order to fuse the semantic information coming from deeper levels of the architecture with the low-level features extracted in the shallower 
levels. Similarly, the U-Net architecture presented in Ronneberger et al., 2015 introduced an encoder-decoder architecture, where the skip connections act as a bridge from the encoding to the decoding path at the corresponding level of the architecture. Similar to these works, the FPNs in object detection (see Fig.2.1d) obtained significant results on the COCO dataset where challenging small objects are present. In this chapter, we inherit the concepts of FCNs, FPNs, and U-Net for building a novel FCN architecture similar to the one shown in Fig. 2.1e.

The algorithms developed for the identification of the elements in overhead power transmission lines have exhibited a similar transition from the use of classical image processing techniques to machine learning methods. SVM classifiers were used in Wang and Zhang, 2016, Prasad and Rao, 2016; Zhao et al., 2016 for insulator identification and analysis. In Wang and Zhang, 2016, Gabor features were used to train an SVM classifier for insulator identification. Qualitative results were only reported for one type of insulator and two aerial images. SVMs were also used for electric tower detection in images of several spectra (visible and infrared) in Martinez et al., 2018. Electric tower detection was also addressed in Sampedro et al., 2014 using HOG for feature extraction in combination with a shallow fully-connected network for classification. In the last five years, machine learning strategies have evolved towards the usage of deep learning techniques, which have proven to provide outstanding results for object detection in multiple applications. For this reason, several authors have addressed the power line inspection problem by using deep learning models: In Gubbi et al., 2017, two models were trained and compared for electric wire detection: a pre-trained CNN based on the GoogLeNet architecture (Szegedy et al., 2015), and a CNN model with 4 convolutional layers where HOG features were used as input to the CNN. In the approach presented in Varghese et al., 2017, GoogLeNet pre-trained network was also used for addressing the detection of several power line components such as wires, electric towers, and insulators, where the output of the CNN model was post-processed using a spectral clustering algorithm. More recently, several authors have addressed the problem of insulator detection by using consolidated object detectors such as Faster R-CNN, YOLOv2 or SSD. The former was used in Gao et al., 2017 and Ling et al., 2018, YOLOv2 detector was utilized in Siddiqui et al., 2018 for detecting different type of insulators (polymer and ceramic). SSD pre-trained using the COCO dataset was used in Miao et al., 2019 to detect composite and ceramic insulators. SSD in combination with deep residual networks was also utilized in Nguyen et al., 2019 for detecting different elements in medium voltage electric towers.

Within the field of deep learning, most recent approaches for insulator string segmentation make use of Semantic Segmentation algorithms (Gao et al., 2017; Ling et al., 2018; Chang et al., 2018). In Gao et al., 2017 and Ling et al., 2018, Faster R-CNN was used for insulator localization in RGB images. After locating the insulator, in Gao et al., 2017 an FCN-8s (Long et al., 2015) allowed the extraction of each disc of the insulator string for posterior diagnosis tasks. Again, only qualitative results were presented in a very limited set of test images, showing inaccurate disc segmentation results. In Ling et al., 2018, U-Net was used for segmenting the pixels of the absent disc unit within the insulator string. A conditional GAN framework was adopted in Chang et al., 2018 for insulator segmentation providing good segmentation results for a large variety of insulator types 
with different material, point of view, and orientation. However, a posterior stage for fault diagnosis was not addressed in their solution.

\subsection{Algorithms based on Region Proposals}

The object detection algorithm presented in this section is designed for the detection of predefined objects whose shape is known in advance (e.g., rectangular). The proposed algorithm is based on the combination of four main modules:

- Region proposal module: This module is responsible for computing the most probable candidates in the image for being the object of interest. In the use case studied in this section, this object corresponds to a helipad, whose rectangular shape is known in advance. Assuming the rectangular shape of the helipad, this module applies a set of operations consisting of extracting the edges in the image using the Canny algorithm (Canny, 1987), and finding rectangular shapes on the resulting image. The latter, will be used as proposals to the supervised learning classifier module, which provides an additional level of robustness to the system.

- Feature extractor: This component is in charge of computing a descriptor vector for each candidate in the image. In the proposed algorithm, we use Histogram of Oriented Gradients (HOG) (Dalal and Triggs, 2005). Thus, In each candidate ROI generated by the region proposal module, HOG features are computed. The configuration of the HOG feature extractor is summarized here:

- Window size: $48 \times 48$ pixels.

○ Block Size: $16 \times 16$ pixels.

- Block Stride: 8 pixels.

○ Cell Size: $8 \times 8$ pixels.

- Histogram bins: 9 bins of 20 degrees each.

The above configuration of the HOG feature extractor leads to a descriptor vector of size $1 \times 900$, which will be passed to the supervised learning classifier.

- Supervised learning classifier: This module acts as the core of the object detection algorithm presented in this section. Its purpose is to classify each of the candidate's descriptor extracted from the previous stage as helipad or background. Two different supervised learning classifiers have been considered: L2 Regularized Logistic Regression (L2R-LR), and Support Vector Machine with linear kernel (L-SVM). The comparative analysis using both classifiers for the task of helipad detection is shown in Section 2.5.4.

- Pose estimator: In order to compute the relative pose of the helipad with respect to the frame of reference of the camera, this module uses a Perspective- $n$-Point $(\mathrm{P} n \mathrm{P})$ algorithm taking as input the corners of the previous computed ROI of the detected 
helipad (image plane), a set of object points (object frame of reference), and the intrinsic parameters and distortion coefficients of the camera. The final computed pose is selected so that it minimizes the reprojection error between the detected points in the image and the projected object points, using an iterative method based on Levenberg-Marquardt optimization and RANSAC for filtering the outliers.

All the previous modules are combined into a single algorithm for helipad detection and altitude estimation. The details of the proposed approach are shown in Algorithm 1 .

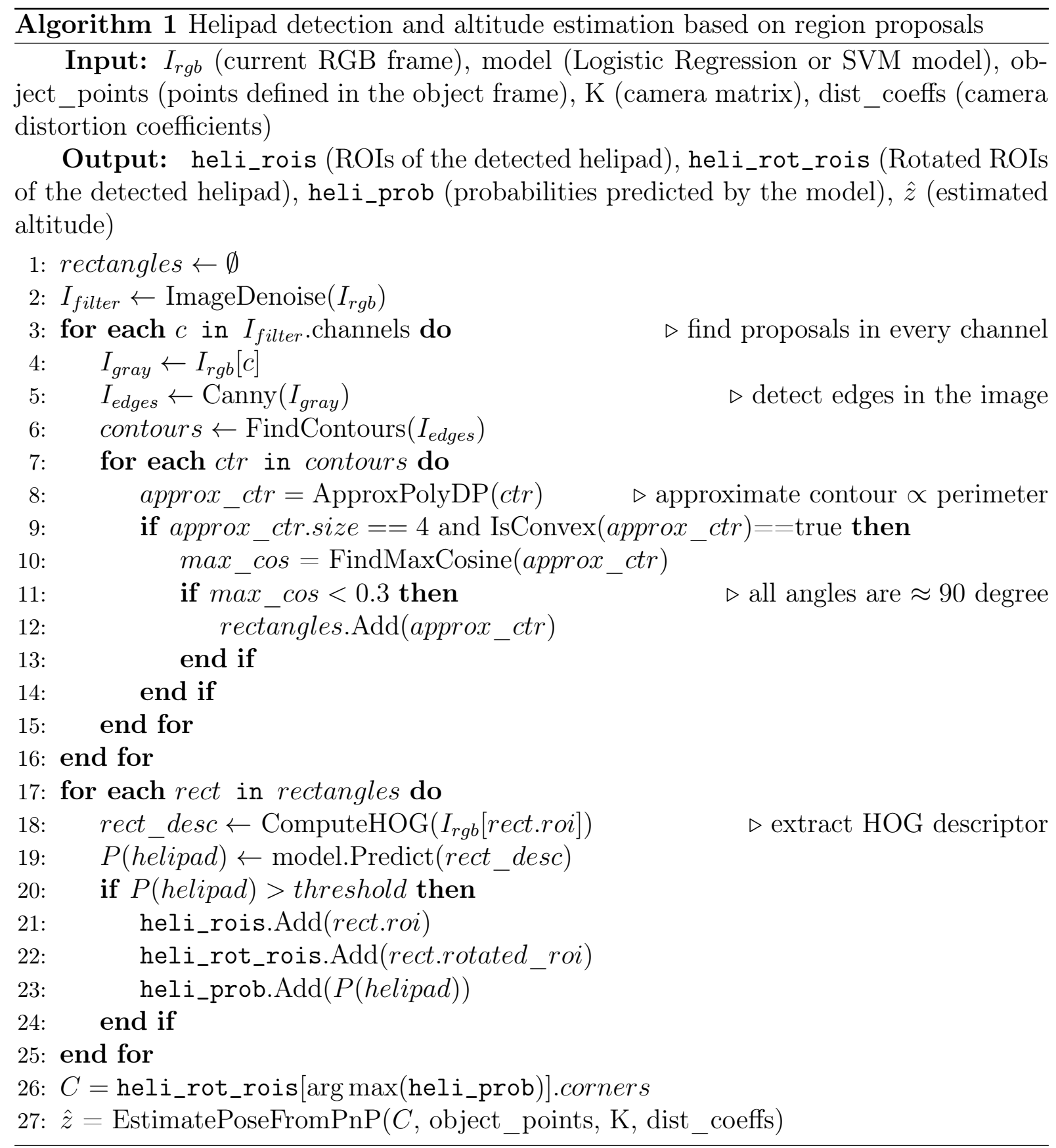


As shown in Algorithm 1, we apply an image denoising stage using a Gaussian filter in order to alleviate the computational operations for subsequent stages (e.g., edge detection). Subsequently, a Canny edge detection algorithm is applied, after which we conduct a find contours stage. The resulting contours are used to find possible candidates in the image. To find these candidates, we first approximate each contour with accuracy proportional to its perimeter using the Douglas-Peucker algorithm. Finally, we compute the possible candidates using the criteria shown in line 9 of Algorithm 1. These criteria take into account the prior knowledge of the helipad which consists of a rectangular region in the image. Thus, the candidate approximated contours should be of size 4 and correspond to a convex polygonal curve. The candidates which satisfy these conditions are stored and passed to the subsequent stages for feature extraction and classification using the supervised learning classifiers considered in this section. Finally, the corners of the rotated bounding box corresponding to the ROI with maximum probability are used by a $\mathrm{P} n \mathrm{P}$ algorithm to compute the estimated altitude of the UAV's bottom camera with respect to the helipad.

\subsection{Algorithms based on Fully Convolutional Networks}

Inspired by the results obtained using Fully Convolutional Networks (FCNs) in semantic segmentation (Long et al., 2015; Ronneberger et al., 2015) and object detection problems (Lin et al., 2017), in this section we explore the design of new FCNs for addressing both problems using the same network architecture. The proposed network, termed Up-Net (see Fig. 2.2), is an adaptation of the state-of-the-art U-Net network in which we add new skip connections at certain levels of the architecture. These skip connections are responsible for fusing more local information extracted by shallower layers, which have smaller receptive fields, with the semantic information extracted from deeper layers. Furthermore, when transfer learning is performed, we add new convolutional layers in the encoding path, following the VGG16 architecture proposed in Simonyan and Zisserman, 2014 .

The proposed Up-Net network was conceived in a preliminary stage as a solution for the insulator string detection problem in a semantic segmentation fashion, where Up-Net is used for segmenting the pixels of the insulator string into the disc or cap classes. The results obtained for the insulator string segmentation are described in detail in Section 2.5.3. Alternatively, we explore the performance of the proposed Up-Net for object detection purposes leveraging the annotations conducted at ROI-level in our previous works (Sampedro et al., 2014; Martinez et al., 2018). The proposed Up-Net network leverages the inherent pyramid architecture of deep convolutional neural networks for obtaining a feature pyramid framework with a minimal extra cost (Lin et al., 2017). The results obtained for electric tower detection are described in Section 2.5.2.

The proposed Up-Net is composed of an encoding and a decoding paths (see Fig. 2.2). When transfer learning is conducted from VGG16 (Up-Net_vgg16), the encoding path follows the VGG16 architecture, which consists of a combination of $3 \times 3$ convolutional layers (padded convolutions) with a stride of 1 and ReLU activation functions (Nair and Hinton, 2010), followed by $2 \times 2$ max-pooling layers with a stride of 2 . After each 


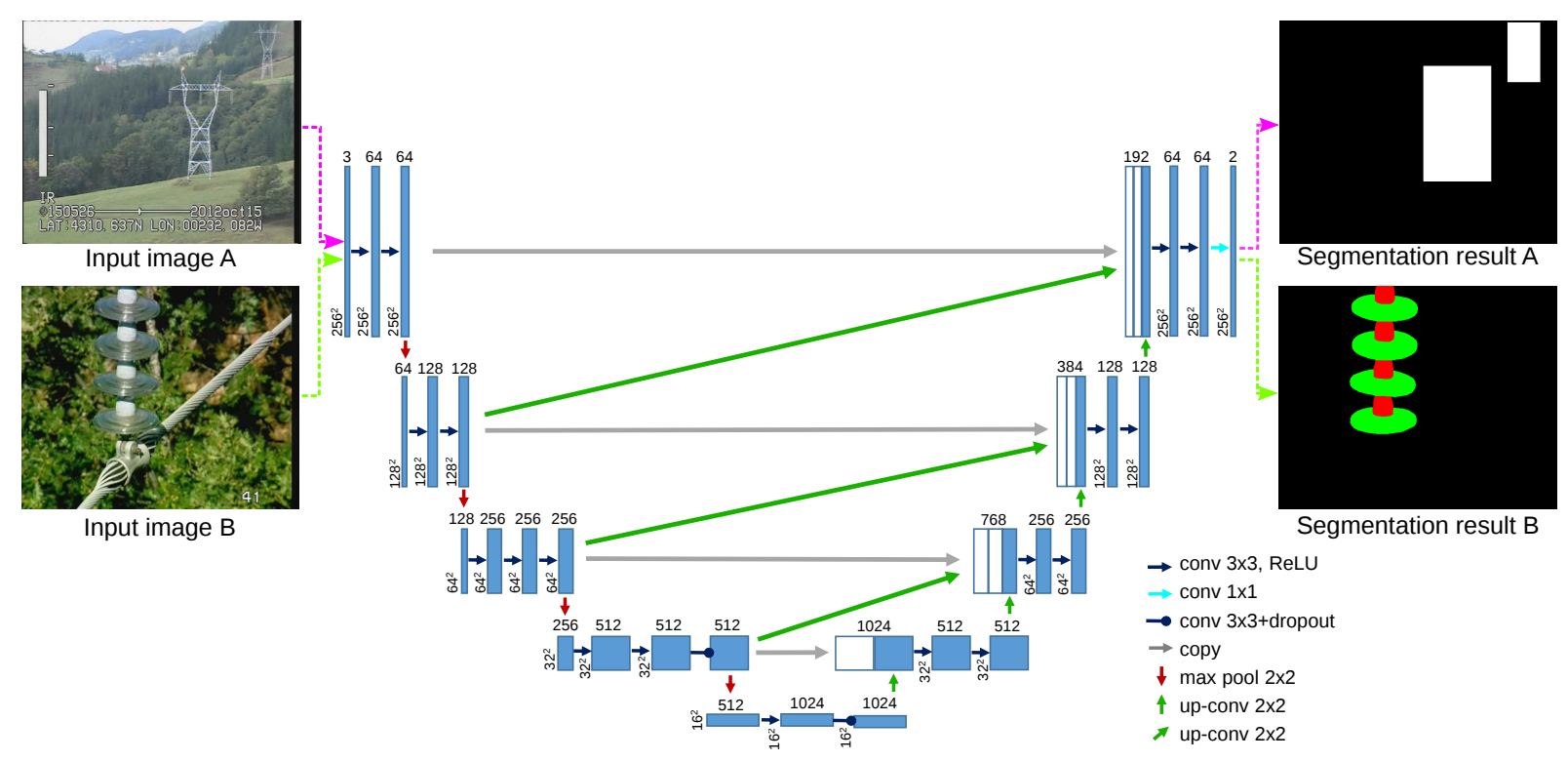

Figure 2.2: Up-Net architecture designed for electric tower detection and insulator string segmentation (best seen in color). The U-Net architecture presented in Ronneberger et al., 2015 is modified in this work by adding new skip connections (green oblique arrows). In addition, when transfer learning is conducted from VGG16, new convolutional layers are added to the encoding path, yielding Up-Net_vgg16 (in the figure) which has $35.45 \mathrm{M}$ parameters. Up-Net takes an RGB image as input and outputs a 1-channel image (for tower segmentation) or 2-channel image (for insulator segmentation) with pixels belonging to cap and disc classes.

pooling operation, the number of channels is increased by a factor of 2 until reaching 512 channels in the fourth convolutional block of the encoding path. The decoding path is nearly symmetric to the encoding one, and consists of a combination of up-sampling and convolutional layers. In the up-sampling layers, a nearest-neighbor interpolation with an up-sampling factor of 2 is applied to double the resolution of the feature maps in the previous level. This up-sampling operation is always followed by $2 \times 2$ convolutional layers ("up-convolution") in order to halve the number of channels, which allows the application of the skip connections to fuse the information coming from the encoding path. Each "up-convolution" is followed by $3 \times 3$ convolutional layers (padded convolutions) with a stride of 1 and ReLU activation functions, mimicking the design of the encoding path. The last layer of the decoding path consists of a $1 \times 1$ convolutional layer in order to reduce the number of feature channels to the number of output labels in the model.

Additionally, we explore an alternative design to the proposed Up-Net_vgg16, considering the hard computational constraints of certain robotic platforms (e.g., Micro Air Vehicles). The shortened version of the Up-Net_vgg16, named Up-Net_tiny, has the same number of layers as the Up-Net_vgg16 model. However, we divide the number of channels in each convolutional layer by 4 . This new design allows obtaining a more efficient network in terms of computational resources, having a total of 2.217 millions of parameters. 


\subsection{Experiments and Results}

In this section, we present the experiments conducted and the results obtained in the evaluation of the electric tower detection and helipad detection methods presented in this chapter. First, the experimental setup is described in Section 2.5.1. Subsequently, the experiments and results obtained in the evaluation of the electric tower detection and insulator string segmentation methods are illustrated in Section 2.5.2 and Section 2.5.3. respectively. Finally, Section 2.5.4 describes the results obtained in helipad detection. Several videos of the reported results can be found in:

- Electric tower detection and insulator string segmentation: https://vimeo.com/ album/5782445

- Helipad detection: https://vimeo.com/356043446

\subsubsection{Experimental Setup}

The L2R-LR and L-SVM models described in Section 2.3 for helipad detection purposes have been trained using LIBLINEAR (Fan et al., 2008) and LIBSVM (Chang and Lin, 2011), respectively, on an Intel Core i7-6700HQ CPU. On the other hand, the networks used for electric tower detection and insulator string segmentation have been implemented in Python and trained using the Keras ${ }^{1}$ and PyTorch ${ }^{2}$ deep learning frameworks. The latter has been used for working with all the models that involve GANs (Goodfellow et al., 2014), while the former has been used for working with the remaining models. These experiments have been conducted on an Nvidia GeForce GTX 1080Ti GPU under Ubuntu 16.04 LTS. OpenCV 4.0.0 $0^{3}$ is used for image processing tasks.

\subsubsection{Experimental Results in Electric Tower Detection}

This section introduces the experiments that have been conducted in order to evaluate the capabilities of the proposed Up-Net network for the task of electric tower detection, and compare its performance to state-of-the-art object detection networks, such as YOLOv2 (Redmon and Farhadi, 2016) and YOLOv3 (Redmon and Farhadi, 2018). Additionally, we analyze the effect of different factors in the performance of the tower detection task, such as:

- Data augmentation: The proposed Up-Net network is trained on the original and augmented datasets.

- Image resolution: The YOLOv2 and YOLOv3 networks have been trained at different resolutions. To this aim, we have considered two image resolutions: $736 \times 576$ pixels, which corresponds practically with the original resolution $(720 \times 576)$, since

\footnotetext{
${ }^{1}$ https://keras.io/

${ }^{2}$ https://pytorch.org/

${ }^{3}$ https://docs.opencv.org/4.0.0/
} 
for training the YOLO variants, the width and height of the image should be divisible by 32 , and $256 \times 256$ pixels, which corresponds to the resolution used for training the proposed Up-Net.

\section{Dataset}

Two different datasets have been collected depending on the type of power line voltage. We summarize here the number of examples utilized for training the models:

- Medium voltage: A total of 4256 images containing medium voltage electric towers have been annotated, within which 4496 medium voltage towers exist. A data augmentation method consisting of a horizontal flip has been conducted, which enables to double the number of training examples, thus obtaining a total of 8512 images.

- High voltage: A total of 1901 images containing high voltage electric towers have been annotated, within which 2000 high voltage towers exist. As in the case of medium voltage, we enlarge the training set by conducting a horizontal flip on every image, which allows obtaining a total of 3802 images for training purposes.

It should be noted that the annotations conducted for electric tower detection have been performed at ROI level, which is defined by the $x, y$ coordinates of the upper-left corner together with the width and height, expressed in pixels. These annotations have been used for training the proposed Up-Net network by converting the ROI label into the corresponding mask image, as shown in the segmentation result for the example A in Fig. 2.2 ,

Regarding the dataset created for evaluation purposes, two test video sequences of medium voltage power distribution lines have been manually annotated as ground truth encompassing 6491 frames. Similarly, two test video sequences related to inspections of high voltage power transmission lines have been manually annotated at ROI level, containing 2806 frames.

\section{Training methodology}

The proposed Up-Net network, and its variant (Up-Net_tiny) used for tower detection purposes have been trained considering a binary classification problem. The output channel given by the output layer computes a sigmoid activation function, where each pixel is assigned to either the tower class or the background class. Thus, a binary crossentropy is used as the loss function, using the Adam optimizer (Kingma and Ba, 2014) for its minimization during a training process of 60 epochs, with a minibatch size of 4 images, and a base learning rate of $10^{-4}$. The rest of the hyperparameters, such as the momentum, are set as described in the original work (Kingma and Ba, 2014). We apply the weight initialization technique proposed in He et al., 2015a, where the initial values of the weights are sampled from a truncated normal distribution centered on 0 and standard deviation of $\sqrt{2 / f a n \_i n}$, where fan_in is the number of input units in the weight tensor. 


\section{Baseline methods}

As mentioned in Section 2.5.2, the performance of the proposed Up-Net network has been compared to several of the best performing models in the state of the art for object detection purposes, such as YOLOv2 and YOLOv3 In the following paragraphs, we provide a brief description of the main features of the baseline methods considered for a better understanding of the results presented in this section.

- YOLOv2 (Redmon and Farhadi, 2016): YOLOv2 for object detection consists of a 30-layer FCN with 22 convolutional and 5 max-pooling layers, which follows the VGG16 architecture by using $3 \times 3$ convolution filters and doubling the number of channels in each level of the network. $1 \times 1$ convolutional layers are also utilized between the $3 \times 3$ convolutional layers to compress the feature representation.

Additional implementation details involve the usage of Batch Normalization (Ioffe and Szegedy, 2015) and five types of anchor boxes extracted using a k-means clustering algorithm with $\mathrm{k}=5$. Regarding the training methodology, YOLOv2 makes use of image resizing every 10 batches to be robust to different resolutions of the input image. Furthermore, several data augmentation techniques are applied, such as random crops and color shifting using the HSV color space.

The shape of the detection kernel is $1 \times 1 \times(B \times(S+C))$, where $B$ stands for the number of boxes $(B=5), S$ is the number of scoring variables per box $(S=5$, using 4 numbers for encoding the bounding box coordinates, and 1 number for the objectness score), and $C$ depicts the number of classes, thus $C=1$ for the specific problem of electric tower detection presented in this section, since we consider a binary classification problem (i.e., tower versus background class). Taking into account the previous configuration, the output volume used for the final detection layer of the YOLOv2 network is of shape $23 \times 18 \times 30$ for the specific tower detection task addressed in this section, considering an input image shape of $736 \times 576 \times 3$.

- YOLOv3 (Redmon and Farhadi, 2018): this network builds on top of its previous version (YOLOv2) by adding state-of-the-art deep neural network elements such as residual connections, skip connections, and up-sampling operations. The network proposed in YOLOv3 is built by stacking convolutional residual blocks (He et al., 2016) until the 75th layer of an encoding path, after which 3 blocks encompassing convolution and up-sampling operations are applied for the detection stage at each of three scales, to get a 106-layer FCN with 75 convolutional layers, where the pooling layers have been substituted by convolutional ones with a stride of 2 . One of the most important features incorporated in YOLOv3 is the prediction of bounding boxes at 3 different scales, following the concept of the Feature Pyramid Networks proposed in Lin et al., 2017.

Additional implementation details involve the usage of 9 anchor boxes extracted using a k-means clustering algorithm. Three for each scale. Multi-scale training, data

\footnotetext{
${ }^{4}$ https://github.com/AlexeyAB/darknet
} 
augmentation based on random crops and color shifting, and Batch Normalization are used as in the case of YOLOv2.

The shape of the detection kernel is $1 \times 1 \times(B \times(S+C))$, where $B=3, S=5$, and $C=1$. Thus, in our problem, the detection kernel is of shape $1 \times 1 \times 18$. Taking into account the previous detection kernel, and considering the 5 down-sampling operations of the encoding path $(\times 32$ down-sampling factor $)$, the output volume for for the final detection layer in the first detection scale is of shape $23 \times 18 \times 18$. The remaining blocks of convolution and up-sampling operations give volumes of $46 \times 36 \times 18$ and $92 \times 72 \times 18$ for the second and third detection scales respectively, considering an input image shape of $736 \times 576 \times 3$.

\section{Evaluation metrics}

The main metric utilized for evaluating the performance of the different models used for object detection is the mean Intersection over Union (mean IoU). This metric is based on the Jaccard index, which provides an estimation of the overlap between two bounding boxes, and is computed using the following expression:

- mean IoU: $(1 / N) \sum_{i} \operatorname{area}\left(A_{i} \cap B_{i}\right) / \operatorname{area}\left(A_{i} \cup B_{i}\right)$

where $A_{i}$ and $B_{i}$ represent the ground truth bounding box and the predicted bounding box at frame $i$, and $N$ is the total number of frames in the corresponding sequence.

Additionally, another important metric commonly used in object detection frameworks is the frames per second (FPS), which provides an estimation of the detection speed of the corresponding model.

As a reference, an object detector is considered as featuring appropriate detection capabilities when the mean IoU is greater than $50 \%-60 \%$ at a frame rate greater than 25 FPS.

\section{Results in tower detection}

As stated previously, several video sequences of real aerial inspections of electric power transmission (high voltage) and distribution (medium voltage) lines have been used for evaluation purposes. The results obtained in the evaluation of the different models used for electric tower detection are depicted in Table 2.1 for the sequences related to medium voltage inspections, and in Table 2.2 for the sequences corresponding to the high voltage inspections.

As shown in Table 2.1, all the models trained for detecting medium voltage electric towers obtain a mean IoU greater than $77 \%$, revealing the accurate detections of the models across the different frames of the sequences. The best performing model in terms of mean IoU is the YOLOv3 trained at the resolution of $736 \times 576$ pixels (res 1 ), which obtains a mean IoU greater than $85 \%$ in both sequences. However, in order to adequately compare the performance of the proposed Up-Net network to the baseline methods, the analysis should be conducted at the resolution of $256 \times 256$ pixels (res2), which is the resolution at which the proposed Up-Net models were trained. At this resolution, the 
proposed Up-Net network, pre-trained using the weights of the VGG16 model and trained on the augmented dataset (Up-Net_vgg16_aug), shows accurate detection capabilities obtaining the same performance as YOLOv2 in sequence $1(81 \%)$, and outperforming both YOLOv2 and YOLOv3 in sequence 2 with a mean IoU of $84.8 \%$. Regarding the processing time, the proposed Up-Net_tiny clearly outperforms the baseline networks, obtaining an average rate greater than 211 FPS in both sequences, and providing at the same time appropriate detection capabilities with mean IoU above $77 \%$ in both sequences.

Table 2.1: Results obtained in the evaluation of the proposed FCN and baseline methods for electric tower detection in two real aerial inspections of medium voltage power lines. Where res 1 stands for the image resolution of $736 \times 576$ pixels, and res 2 corresponds to $256 \times 256$ pixels. Best results are indicated in bold.

\begin{tabular}{ccccc}
\hline Sequence & \#frames & Method & mean IoU (\%) & Avg. FPS \\
\hline \multirow{4}{*}{ Seq1 } & Up-Net_vgg16 & 79.63 & 47.56 \\
& & Up-Net_vgg16_aug & 81.00 & 47.56 \\
& Up-Net_tiny & 77.65 & 211.99 \\
& & Up-Net_tiny_aug & 78.83 & $\mathbf{2 1 1 . 9 9}$ \\
& YOLOv2_res1 & 78.95 & 47.27 \\
& YOLOv2_res2 & 81.02 & $\mathbf{1 2 4 . 3 9}$ \\
& YOLOv3_res1 & $\mathbf{8 5 . 7 7}$ & 30.74 \\
& YOLOv3_res2 & $\mathbf{8 3 . 0 5}$ & 86.26 \\
\hline & Up-Net_vgg16 & 82.67 & 46.49 \\
Seq2 & Up-Net_vgg16_aug & $\mathbf{8 4 . 8 1}$ & 46.49 \\
& Up-Net_tiny & 78.03 & 218.02 \\
& & Up-Net_tiny_aug & 81.86 & $\mathbf{2 1 8 . 0 2}$ \\
& YOLOv2_res1 & 78.40 & 47.27 \\
& YOLOv2_res2 & 82.01 & $\mathbf{1 2 6 . 8 3}$ \\
& YOLOv3_res1 & $\mathbf{8 5 . 0 1}$ & 30.74 \\
& YOLOv3_res2 & 84.24 & 88.61 \\
\hline
\end{tabular}

The results concerning the detection of high voltage electric towers are shown in Table 2.2. As can be seen in Table 2.2, all the models obtain a mean IoU greater than $68 \%$, being the proposed Up-Net_vgg16_aug the one showing the best performance in terms of accuracy, with a mean IoU greater than $85 \%$ in both sequences. The performance of the YOLOv3_res2 model is also remarkable, obtaining a mean IoU greater than $82 \%$ in both sequences. Regarding the processing time, the results shown in Table 2.2 reveal that the proposed Up-Net_tiny and the YOLOv2_res2 are the ones that exhibit the best capabilities as in the case of medium voltage tower detection, since these models are the ones with less number of parameters.

Overall, the results presented in Table 2.1 and 2.2 demonstrate the good performance obtained by the proposed networks for electric tower detection. The analysis of these results reveals the influence of the data augmentation techniques. Training the proposed Up-Net variants using the augmented dataset produces an increment in mean IoU from $1 \%$ 
to $4 \%$ in the sequences of medium voltage inspections and $2.5 \%$ to $10 \%$ in the sequences of high voltage inspections. Regarding the processing time, the proposed networks exhibit real-time processing capabilities with an average processing time of 47.2 FPS for the Up-Net_vgg16 variants, and 208.5 FPS for the Up-Net_tiny variants. The latter model could be of special interest in systems with very limited resources (e.g., Micro Air Vehicles) since this model has 2.22 million parameters with the consequent savings in memory and processing time consumption.

Table 2.2: Results obtained in the evaluation of the proposed FCN and baseline methods for electric tower detection in two real aerial inspections of high voltage power lines. Where res 1 stands for $736 \times 576$ pixels, and res 2 corresponds to $256 \times 256$ pixels. Best results are indicated in bold.

\begin{tabular}{|c|c|c|c|c|}
\hline Sequence & \#frames & Method & mean IoU (\%) & Avg. FPS \\
\hline \multirow{6}{*}{ Seq1 } & \multirow{6}{*}{2318} & Up-Net_vgg16 & 82.97 & 47.30 \\
\hline & & Up-Net_vgg16_aug & 85.63 & 47.30 \\
\hline & & Up-Net_tiny & 78.58 & 193.05 \\
\hline & & Up-Net_tiny_aug & 81.09 & 193.05 \\
\hline & & YOLOv2_res1 & 80.03 & 47.27 \\
\hline & & YOLOv2_res2 & 80.00 & 116.78 \\
\hline \multirow{10}{*}{ Seq2 } & \multirow{10}{*}{488} & YOLOv3_res1 & 85.00 & 30.74 \\
\hline & & YOLOv3_res2 & 82.64 & 88.51 \\
\hline & & Up-Net_vgg16 & 75.51 & 47.49 \\
\hline & & Up-Net_vgg16_aug & 85.32 & 47.49 \\
\hline & & Up-Net_tiny & 68.37 & 210.90 \\
\hline & & Up-Net_tiny_aug & 77.12 & 210.90 \\
\hline & & YOLOv2_res1 & 69.31 & 47.27 \\
\hline & & YOLOv2_res2 & 76.70 & 117.15 \\
\hline & & YOLOv3_res1 & 81.93 & 30.74 \\
\hline & & YOLOv3_res2 & 82.37 & 88.39 \\
\hline
\end{tabular}

Qualitative results showing the detection of the best performing models from Table 2.1 and 2.2 are shown in Fig. 2.3 and 2.4 for the medium voltage and high voltage sequences, respectively. As shown in Fig. 2.3, the proposed Up-Net_vgg16 network is able to detect different types of medium voltage electric towers with varying shape and size. The two images in the center of Fig. $2.3 \mathrm{~b}$ show the robustness of the proposed detector under a wide range of sizes of the electric tower in the image. Furthermore, the results presented in Fig. 2.3 reveal the robust detection capabilities under different lighting conditions and backgrounds. Similarly, the results presented in Fig. 2.4 validate the detection capabilities of the proposed Up-Net_vgg16 in high voltage power line inspections. As can been seen in Fig. 2.4a, sequence $\overline{1}$ is composed of different types of high voltage electric towers which appear in the image with very different shapes and points of view (see last image in Fig. 2.4a). Despite this variability, the proposed network is able to provide accurate detection results. 

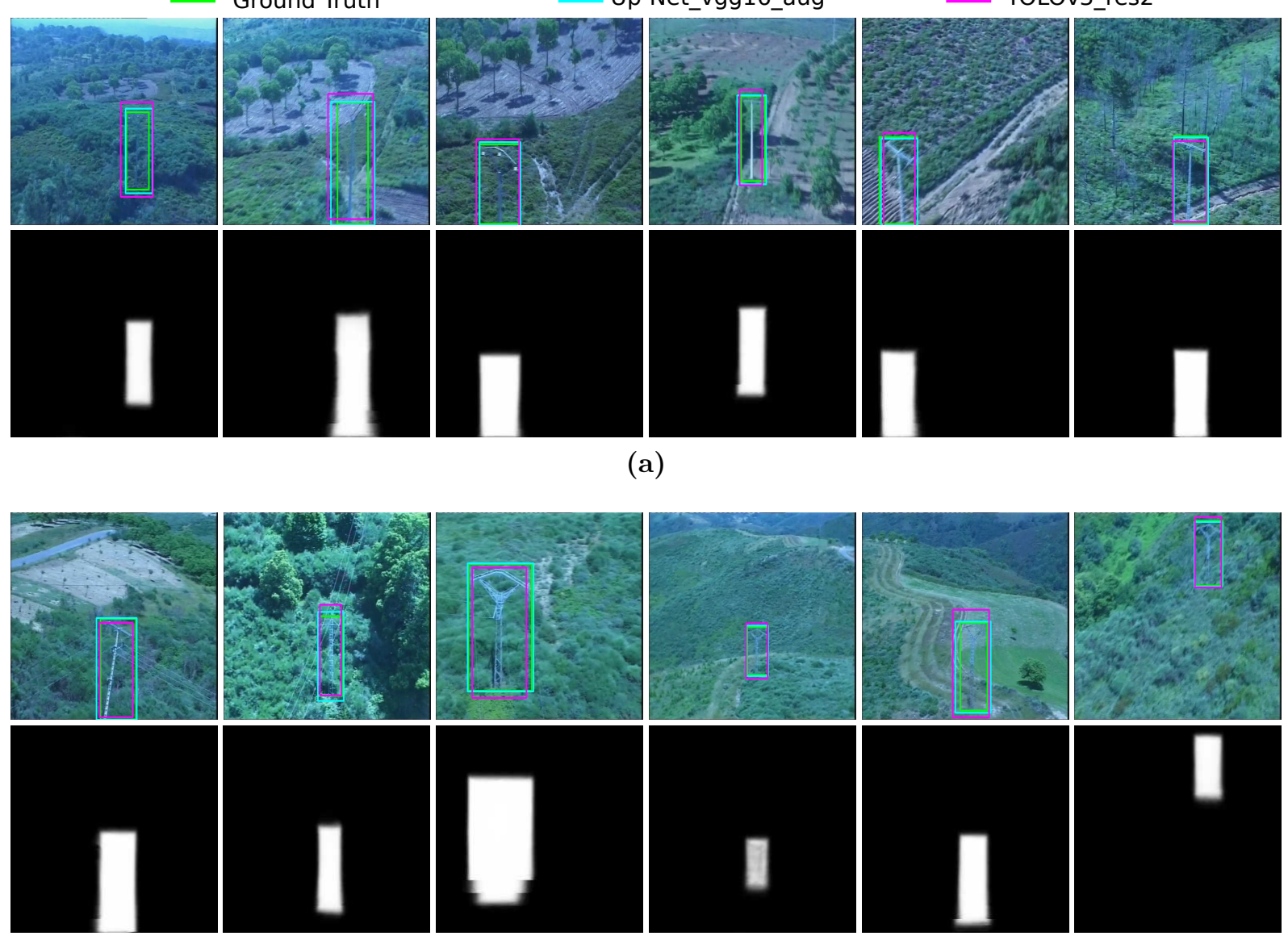

(b)

Figure 2.3: Tower detection results obtained in several sequences related to inspections of medium voltage power lines (best seen in color). (a) Detection results obtained in sequence 1 and masks predicted by the proposed Up-Net_vgg16_aug. (b) Results obtained in sequence 2 and masks predicted by the proposed Up-Net_vgg16_aug.

Regarding the output mask predicted by the proposed Up-Net_vgg16 network, several examples are shown in the second rows of Fig. 2.3 and 2.4. As shown in the last two images of Fig. 2.4, the proposed Up-Net_vgg16 network is able to generate reasonable outputs even when the electric tower appears partially in the image and has a considerable size. It should be noted that these types of images have not been used in the training set, which is mainly composed of images of electric towers which appear entirely in the image.

\subsubsection{Experimental Results in Electric Insulator Segmentation}

This section presents the experiments that have been conducted in order to select the most appropriate model for the insulator string segmentation task. For this purpose, several state-of-the-art semantic segmentation networks have been evaluated and compared to the proposed Up-Net architecture. In addition, a thorough analysis has been conducted for studying the effects of three main aspects: 

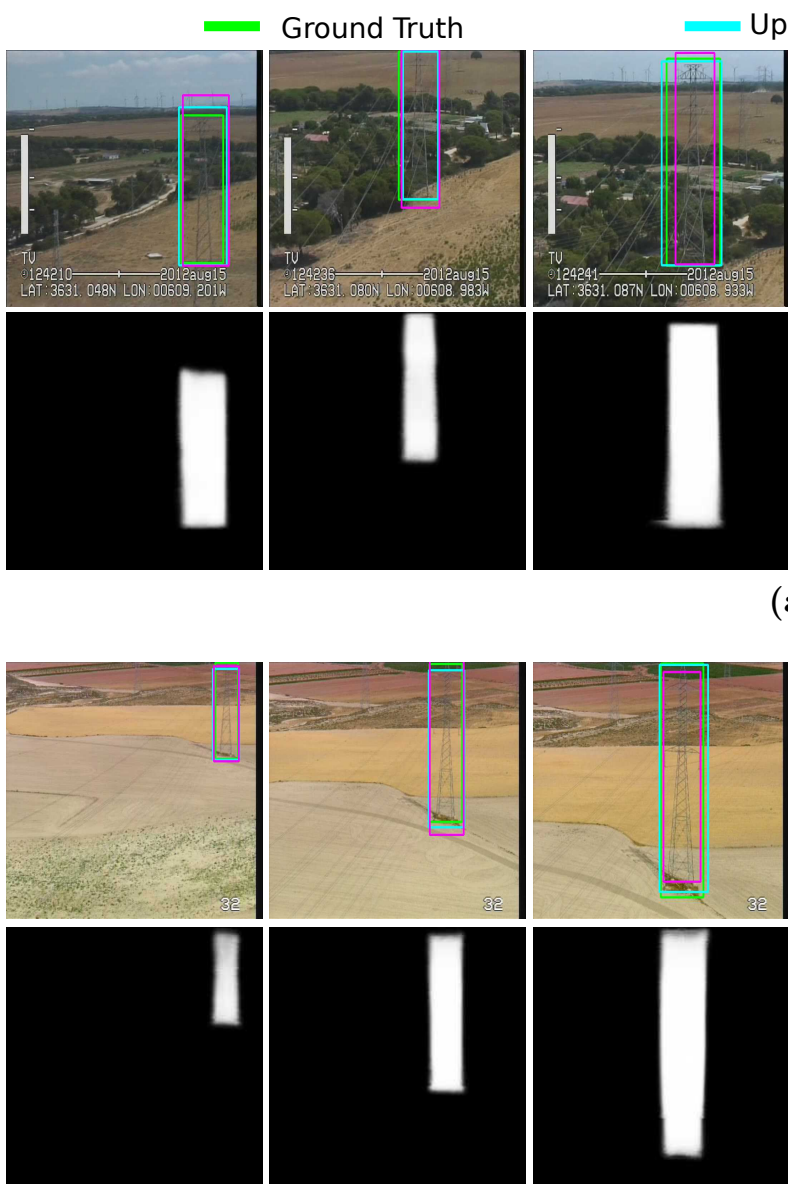

(b)

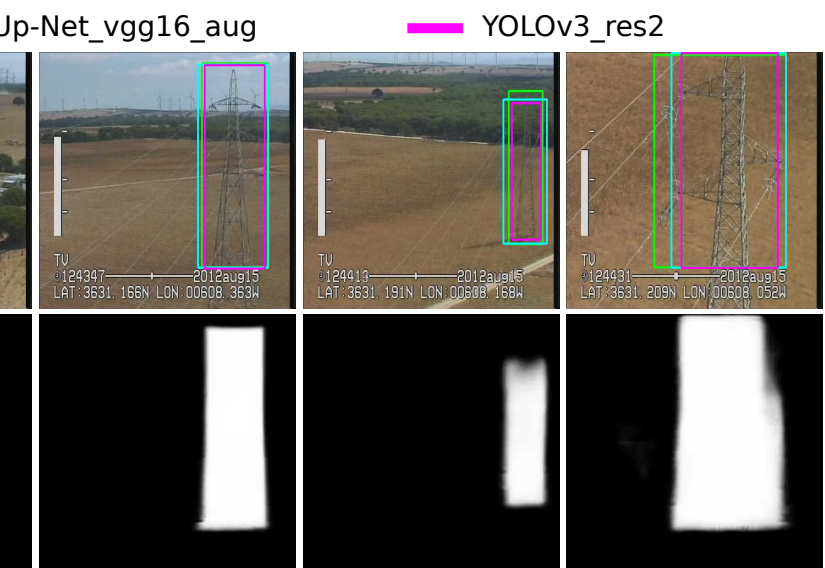

(a)
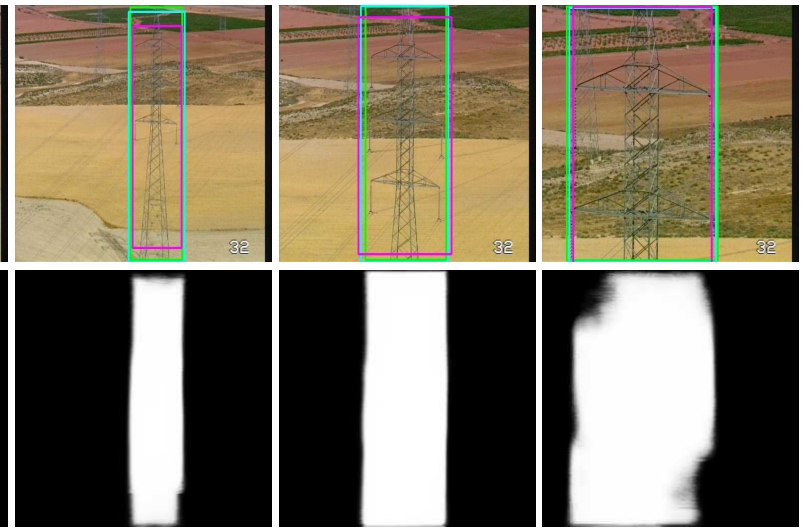

Figure 2.4: Tower detection results obtained in several sequences related to inspections of high voltage power lines (best seen in color). (a) Detection results obtained in sequence 1 and masks predicted by the proposed Up-Net_vgg16_aug. (b) Results obtained in sequence 2 and masks predicted by the proposed Up-Net_vgg16_aug.

- Data augmentation: all the networks have been trained using the original dataset composed of 160 images, and an augmented dataset containing 640 images.

- Transfer learning: most of the networks evaluated in this section have been trained after transferring the weights of the VGG16 network previously trained on the ImageNet database (Deng et al., 2009).

- Generative adversarial networks: we evaluate the performance of state-of-the-art conditional GANs applied to the insulator string segmentation task. In addition, we integrate the U-Net and Up-Net networks within a conditional GAN framework (GAN_U-Net and GAN_Up-Net) in order to study its influence. In this case, no transfer learning is performed in order to properly compare to other baseline networks involving GANs. 


\section{Dataset}

In order to train and validate all the models implemented for the task of insulator string segmentation, a total of 160 images of $720 \times 576$ pixels were manually annotated, where the pixels of the insulator string were assigned to the disc or cap class. Some examples of the original images and the ground truth annotations can be reviewed in Fig. 2.5. In order to analyze the effect of the number of training samples in each evaluated model, and taking into account the characteristics of the images that make up the original dataset, in this work two main data augmentation techniques have been considered. First, all the images have been augmented by a horizontal flip, thus doubling the number of images in the dataset. Subsequently, a gamma correction technique is applied to the original image by applying a lookup table (LUT), where each value (pixel intensity) in the table is computed using the expression: $L U T[i]=(i / 255)^{\gamma} \times 255$, where $i$ ranges from 0 to 255 and we clip the previous expression to be within the range $[0,255]$. For each image in the original dataset, two random values of gamma were picked from the range $[0.4,0.8]$ for the first value and $[1.2,2.5]$ for the second. Using the aforementioned data augmentation techniques, the original dataset was augmented by a factor of 4 , obtaining a total of 640 images (augmented dataset).
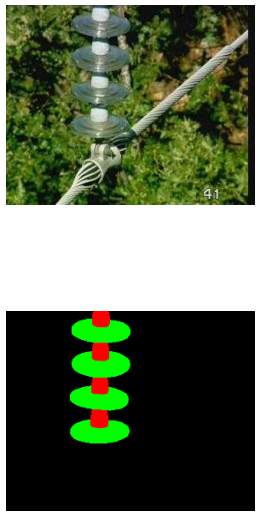
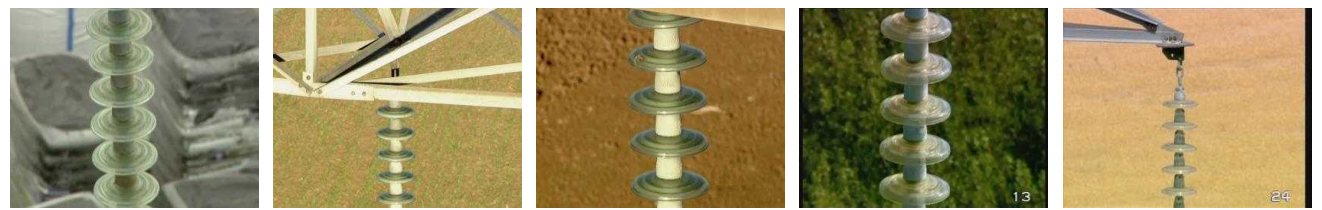

(a)
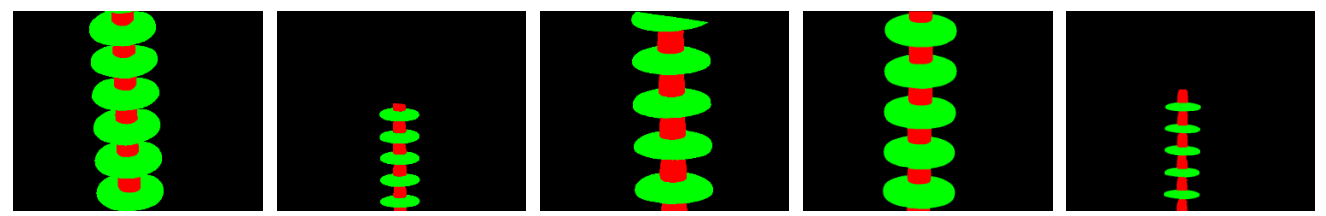

(b)

Figure 2.5: Examples of images used for training the insulator string segmentation models. (a) Original RGB images. (b) Ground truth annotations. The pixels of the insulator string are assigned to the disc (green) or cap (red) class.

In order to perform a detailed evaluation and comparison of all the networks presented in this section, a test set of 240 images was created by manually annotating the pixels of the insulator string to the disc or cap class. This test set includes images from a similar domain as the ones presented in the training set, and 75 images (i.e., 31\%) with a completely different domain, which were downloaded from the Internet.

\section{Training methodology}

All the networks for insulator segmentation purposes have been trained by considering a binary classification problem for each of the two classes (i.e., disc or cap), where each 
of the two channels of the output layer of the corresponding network computes a sigmoid function which models the probability of the corresponding pixel as belonging to disc or cap class versus the background class. Thus, a binary cross-entropy is used as the loss function, using the Adam optimizer for its minimization during a training process of 60 epochs and a minibatch size of 4 images. The learning rate used in the Adam optimizer has been empirically found for each network using a grid search procedure, being $10^{-4}$ for the proposed Up-Net and GAN_Up-Net networks. The rest of the hyperparameters are set as described in the original work (Kingma and Ba, 2014). We apply the weight initialization technique proposed in He et al., 2015a where the initial values of the weights are sampled from a truncated normal distribution centered on 0 and standard deviation of $\sqrt{2 / f a n_{-} i n}$, where fan_in is the number of input units in the weight tensor.

Inspired by the results obtained in Isola et al., 2017; Chang et al., 2018, we also train the best performing models under the conditional GAN framework proposed in pix2pix (Isola et al., 2017). GANs are generative models which learn a mapping function from a random noise vector to an output image $(G: \mathbf{z} \mapsto \mathbf{y})$. Conversely, in the conditional GAN framework, the mapping is learned from a random noise vector and an input image $(G:\{\mathbf{x}, \mathbf{z}\} \mapsto \mathbf{y})$.

The loss functions of the generator and discriminator used for training the conditional GAN models are computed using Eq. 2.1 and Eq. 2.2, respectively. As can be seen in Eq. 2.1, the loss function encompasses two terms. The first term implements an L1 operation, aiming at modeling the low-frequency part of the desired output. The second term is intended to model the high-frequency part of the signal (edges and complex details) and is learned using a second network (discriminator). This new network is trained using Eq.2.2 in order to distinguish ground truth labels from the ones generated by the generator network. During training, the two networks (generator and discriminator) take turns to update their parameters. We have found that performing a training step every time a batch is sampled is the most appropriate method for obtaining a correct convergence in the specific task of insulator string segmentation presented in this work.

$$
\begin{array}{r}
\mathcal{L}_{G E N}(G, D)=\mathbb{E}_{\mathbf{x}, \mathbf{y}, \mathbf{z}}\left[\|\mathbf{y}-G(\mathbf{x}, \mathbf{z})\|_{1}\right]+ \\
\alpha\left(\mathbb{E}_{\mathbf{x}, \mathbf{z}}[\log (D(\mathbf{x}, G(\mathbf{x}, \mathbf{z})))]\right) \\
\mathcal{L}_{D I S}(G, D)=\mathbb{E}_{\mathbf{x}, \mathbf{y}}[\log D(\mathbf{x}, \mathbf{y})]+ \\
\mathbb{E}_{\mathbf{x}, \mathbf{z}}[\log (1-D(\mathbf{x}, G(\mathbf{x}, \mathbf{z})))]
\end{array}
$$

where $G$ and $D$ denote the generator and discriminator networks respectively, $\mathbf{x}$ and $\mathbf{y}$ represent the input RGB image and the desired output mask respectively, and $\mathbf{z}$ is a random noise vector. The parameter $\alpha$ in Eq. 2.1 models the influence of the conditional GAN framework. This parameter has been selected by performing a grid search over a wide range of values $(\alpha \in[0.01,50])$, obtaining an optimal value of $\alpha=0.1$.

In all the experiments, the images of the corresponding dataset (i.e., original or augmented) are resized to $256 \times 256$ pixels and randomly divided into train and validation sets with a percentage of samples of $80 \%$ and $20 \%$, respectively. 


\section{Baseline methods}

A detailed evaluation of the proposed method for segmenting insulator strings has been conducted by comparing its performance to several state-of-the-art algorithms which have proven to provide outstanding results in different semantic segmentation problems. In the next paragraphs, we provide a brief description of the implementation details of the different baseline approaches considered:

- Fully Convolutional Networks (FCNs): we implement the FCN-8s model presented in Long et al., 2015 with the base architecture of the VGG16 network and adding the skip connections described in the original work which allow fusing the information from the last convolutional layer (conv7) with the information of lower levels in the architecture (pool4 and pool3). This is done by applying $4 \times$ and $2 \times$ upsamplings using deconvolution layers. In addition, we experimented with several versions of the FCN-8s model with and without L2 regularization with a decay of $2^{-4}$. The resulting model has $134.27 \mathrm{M}$ parameters. The Adam optimizer with a base learning rate of $10^{-4}$ is used for training the $\mathrm{FCN}-8$ s network.

- SegNet: this network follows the implementation described in Badrinarayanan et al., 2017, which consists of an encoder-decoder architecture, in which the encoder is based on the VGG16 architecture with 13 convolutional layers, each of one having its analogous convolutional layer in the 13-layer decoding network, yielding a model of 29.46M parameters. Each convolutional layer in the encoder and decoder networks is followed by a Batch Normalization layer after which a ReLU non-linearity is applied. As described in the original work, the upsampling operations are performed by using the memorized pooling indices from the max-pooling layers of the encoder network. In the case of the SegNet model, the most suitable learning rate for the Adam optimizer has been empirically obtained, being $5^{-5}$.

- Convolutional Autoencoder (CAE): the topology of this model follows the encoderdecoder architecture of the SegNet network with the only difference being that in this case we do not use the pooling indices from the encoder network but instead the upsampling operations use a nearest-neighbor interpolation. Thus, the number of parameters of this model is the same as the SegNet architecture. This model is trained using a base learning rate of $10^{-3}$ for the Adam optimizer.

- U-Net: in this case, two types of architectures have been implemented depending on whether transfer learning is carried out or not. The first architecture in which transfer learning is not applied, named U-Net as the original work, follows the architecture described in Ronneberger et al., 2015, having about 31M parameters. The only difference with the original U-Net architecture is the application of padded convolutions, and two 50\% dropout layers (Srivastava et al., 2014) in the fourth and fifth convolutional blocks. In the second architecture, termed U-Net_vgg16, we perform transfer learning from the VGG16 network trained on the ImageNet database (Deng et al., 2009). In this case, the original encoder network from the U-Net architecture is extended from 10 to 12 convolutional layers, yielding an architecture with 
33.98M parameters, where the first 10 convolutional layers are identical to those of the VGG16 network. All the networks consisting of a U-Net-based architecture are trained considering a base learning rate of $10^{-4}$ for the Adam optimizer.

The implementation details of the baseline models trained within a GAN framework are summarized in the next paragraphs.

- ISNet: we implement the conditional GAN network described in Chang et al., 2018, having a generator with $9.65 \mathrm{M}$ parameters. The implementation follows closely the one explained in the original paper with two main differences. First, we only perform a single-stage training instead of the two-stage training finally suggested by the authors, and second, we perform a grid search of all the parameters (not provided by the authors) as described in Section 2.5.3.

- pix2pix: this model follows the generator-discriminator architecture presented in Isola et al., 2017, where the generator consists of a U-Net architecture and the discriminator is based on an $N \times N$ "PatchGAN", in which the discriminator is responsible for distinguishing between real and fake image patches. In this case, we use directly the released cod $\biguplus^{5}$ provided by the authors, in which they include several improvements as compared to the original paper. First, a least squares GAN framework (Mao et al., 2017) is implemented, which substitutes the loss function in Eq. 2.1, and second, a deeper version of the U-Net generator is included, yielding a network with $54.41 \mathrm{M}$ parameters. A minimal change was made for training the pix2pix model, consisting of deactivating the data augmentation per batch in order to properly compare to the other semantic segmentation models.

\section{Evaluation metrics}

The metrics used to evaluate the different insulator string segmentation networks are taken from Long et al., 2015, and are summarized here for a better understanding of the results obtained:

- Pixel accuracy: $\sum_{i} n_{i i} / \sum_{i} t_{i}$

- Mean accuracy: $\left(1 / n_{c l}\right) \sum_{i}\left(n_{i i} / t_{i}\right)$

- Mean Intersection over Union (Mean IoU):

$$
\left(1 / n_{c l}\right) \sum_{i} n_{i i} /\left(t_{i}+\sum_{j} n_{j i}-n_{i i}\right)
$$

- Frequency weighted IoU (f.w. IoU):

$$
\left(\sum_{k} t_{k}\right)^{-1} \sum_{i} t_{i} n_{i i} /\left(t_{i}+\sum_{j} n_{j i}-n_{i i}\right)
$$

where $n_{c l}$ represents the number of classes included in the ground truth segmentation, $n_{i j}$ is the number of pixels of class $i$ predicted to belong to class $j$, and $t_{i}$ is the total number of pixels of class $i$ in the ground truth segmentation. In this work, we consider $n_{c l}=3$, including the disc, cap and background classes.

\footnotetext{
${ }^{5}$ https://github.com/junyanz/pytorch-CycleGAN-and-pix2pix
} 


\section{Results in insulator string segmentation}

The results obtained in the evaluation of the insulator string segmentation networks are shown in Table 2.3 when training the models on the original and augmented datasets. Several trainings (from 5 to 10) have been conducted for each network in order to select the most appropriate set of hyperparameters. The results obtained by the best models are shown in Table 2.3 .

Table 2.3: Insulator string segmentation results on the test set composed of 240 images, resized to $256 \times 256$ pixels, when training on the original and augmented datasets. Metrics are expressed in percentage. Networks whose name ends in "vgg16" are pre-trained with the weights of the VGG16 network trained on ImageNet.

\begin{tabular}{|c|c|c|c|c|c|c|c|c|c|c|}
\hline \multirow{2}{*}{ Model } & \multicolumn{4}{|c|}{ Original dataset } & \multicolumn{4}{|c|}{ Augmented dataset } & \multirow{2}{*}{$\begin{array}{c}\text { \#param } \\
(\mathrm{M})\end{array}$} & \multirow{2}{*}{$\begin{array}{l}\text { Infer. time } \\
\text { (ms/image) }\end{array}$} \\
\hline & Pixel Acc. & Mean Acc. & Mean IoU & f.w. IoU & Pixel Acc. & Mean Acc. & Mean IoU & f.w. IoU & & \\
\hline FCN-8s & 94.60 & 80.51 & 72.65 & 90.64 & 96.04 & 85.19 & 78.98 & 92.81 & 134.27 & 52.07 \\
\hline FCN-8s_vgg16 & 96.84 & 87.56 & 83.47 & 94.08 & 97.81 & 90.36 & 86.87 & 95.84 & 134.27 & 51.75 \\
\hline SegNet & 94.52 & 81.59 & 76.26 & 90.19 & 95.49 & 85.23 & 80.48 & 91.89 & 29.46 & 29.12 \\
\hline SegNet_vgg16 & 97.99 & 92.09 & 88.53 & 96.21 & 98.07 & 93.64 & 90.19 & 96.41 & 29.46 & 29.37 \\
\hline $\mathrm{CA} \overline{\mathrm{AE}}$ & 96.34 & 85.82 & 79.98 & 93.33 & 96.83 & 88.28 & 83.07 & 94.23 & 29.46 & 14.42 \\
\hline CAE_vgg16 & 97.04 & 90.02 & 83.95 & 94.57 & 97.45 & 91.07 & 85.85 & 95.30 & 29.46 & 14.41 \\
\hline U-Net & 96.25 & 87.98 & 81.80 & 93.26 & 96.77 & 89.24 & 85.44 & 94.00 & 31.03 & 14.91 \\
\hline U-Net_vgg16 & 98.19 & 94.14 & 90.41 & 96.58 & 98.20 & 93.98 & 90.94 & 96.60 & 33.98 & 15.67 \\
\hline Up-Net_vgg16 & 98.21 & 93.65 & 90.31 & 96.60 & 98.58 & 95.16 & 92.06 & 97.30 & 35.45 & 19.28 \\
\hline ISNet & 95.91 & 87.67 & 79.10 & 92.73 & 97.35 & 91.56 & 85.77 & 95.11 & 9.65 & 4.52 \\
\hline pix2pix & 97.32 & 90.00 & 84.71 & 95.05 & 97.20 & 91.20 & 84.60 & 95.00 & 54.41 & 10.95 \\
\hline GAN_U-Net & 96.27 & 89.45 & 81.42 & 93.38 & 97.99 & 93.78 & 88.70 & 96.32 & 31.03 & 10.81 \\
\hline GAN_Up-Net & 97.01 & 91.05 & 84.84 & 94.60 & 98.24 & 94.60 & 90.03 & 96.73 & 32.50 & 14.72 \\
\hline
\end{tabular}

As shown in Table 2.3, the networks that integrate into their architecture the proposed Up-Net topology (i.e., Up-Net_vgg16 and GAN_Up-Net), are the ones that obtain the best performance in the task of insulator string segmentation when training on the augmented dataset. The transition from the original to the augmented dataset shown in Table 2.3 reveals the huge impact in performance produced by the application of data augmentation. The proposed data augmentation strategy clearly benefits networks without pre-training (e.g., FCN-8s and SegNet where the mean accuracy is improved by $4.7 \%$ and $3.6 \%$, and the mean IoU is improved by $6.3 \%$ and $4.2 \%$, respectively), and is remarkable in the networks GAN_U-Net and GAN_Up-Net in which the mean accuracy is improved by $4.3 \%$ and $3.6 \%$, and the mean IoU is improved by $7.3 \%$ and $5.2 \%$, respectively. It is interesting to note that although in most of the networks the increment in performance is noticeable when training on the augmented dataset, in others the improvement is very small, as in the case of U-Net_vgg16 and pix2pix.

On the other hand, the application of transfer learning provides the most significant improvement in performance across all the networks considered. As shown in Table 2.3. the mean accuracy of the networks FCN-8s, SegNet, and U-Net is increased by $7 \%, 10.5 \%$ and $6.2 \%$, and the mean IoU is improved by $10.8 \%, 12.3 \%$ and $8.6 \%$ respectively when the networks are pre-trained using the weights of the VGG16 previously trained on ImageNet.

Qualitative segmentation results on a subset of images from the test set are illustrated in Fig. 2.6 and 2.7. This subset of images is quite representative as it encompasses insulators made of different materials (i.e., glass and ceramic), different points of view, 
backgrounds, presence of shadows and reflections on the surface of the insulator, etc. As shown in Fig. 2.6 and 2.7, most of the networks provide accurate semantic segmentation results, being the most challenging images the second (i.e., ceramic insulator with shadows) and sixth (i.e., a tilted insulator with shadows). Fig. 2.7 shows the results of the networks trained within a conditional GAN framework, where it can be clearly observed how these networks tend to produce more noisy segmentation results, in which false positives, related to the structure of the electric tower, appear for most of the networks.

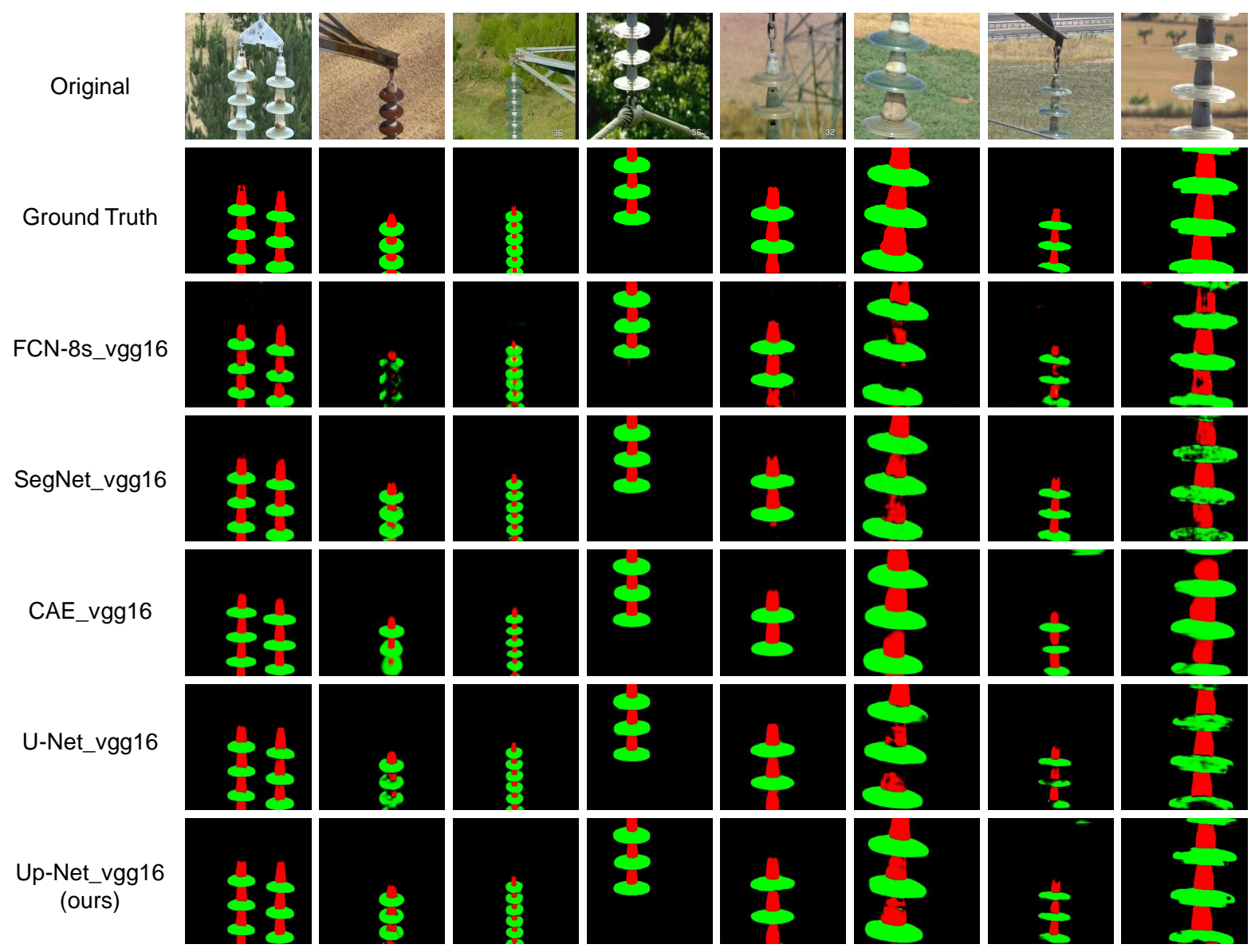

Figure 2.6: Insulator string segmentation results on a subset of images from the test set obtained by the networks trained without a conditional GAN framework.

Taking into account the results presented in Table 2.3, the network Up-Net_vgg16 is selected as the most appropriate for the insulator string segmentation task. For this purpose, and in order to be robust to different insulator string orientations, this network is re-trained on a second augmented dataset. This new augmentation involves the application of two random rotations between the range $\left[30^{\circ}, 60^{\circ}\right]$ and $\left[-60^{\circ},-30^{\circ}\right]$ respectively for each of the 640 images of the augmented dataset, yielding a total of 1920 images. We have observed that training the Up-Net_vgg16 network using this second augmented dataset does not worsen the ability to segment vertical insulator strings and provides the appropriate capabilities for segmenting insulator strings in various orientations. 


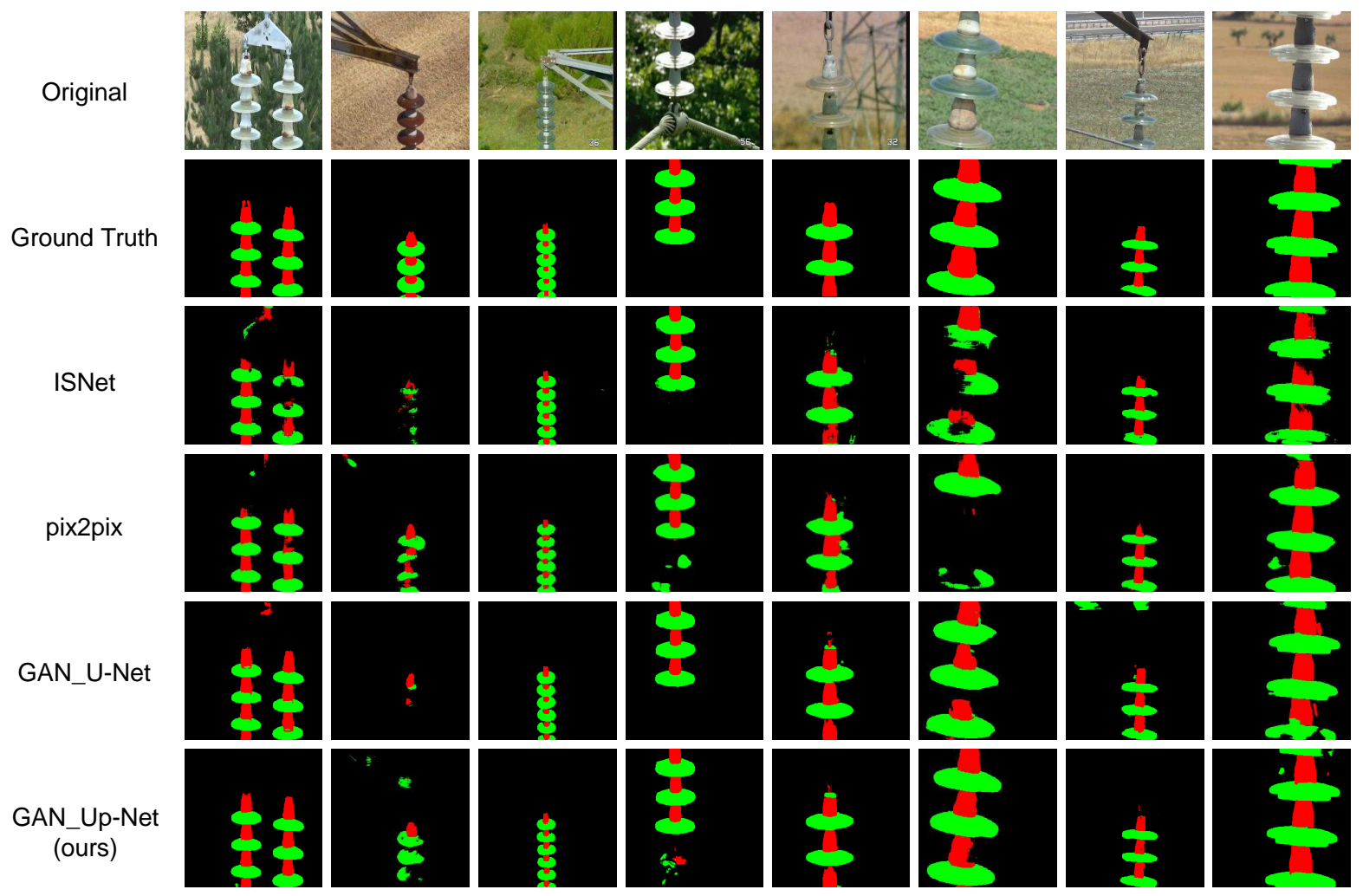

Figure 2.7: Insulator string segmentation results on a subset of images from the test set obtained by the networks trained within a conditional GAN framework.

\subsubsection{Experimental Results in Helipad Detection}

In this section, we present the experiments and results that have been conducted in order to evaluate the proposed helipad detection method, comparing its performance to consolidated state-of-the-art object detectors such as YOLOv2 and YOLOv3. The sequences used for the evaluation have been selected for conducting a detailed analysis of the following aspects:

- Image variability: The test sequences have been captured in indoor and outdoor environments with very variable lighting conditions. Furthermore, two of the sequences have been obtained during real flights, representing a big challenge owing to the vibrations of the aerial robot during the flight. These vibrations generate artifacts in the image, which can significantly complicate the task of object detection.

- Object size: The experiments presented in this section have been carried out to faithfully simulate the conditions that may occur in autonomous landing missions under real operating conditions. To this aim, in two of the sequences, the UAV has been flown until 8-11 m height, causing the helipad to appear with a considerably small size in the image. 


\section{Dataset}

The dataset used for training the proposed helipad detection and baseline algorithms is composed of 7200 images of $640 \times 480$ pixels, equally distributed between the background and the helipad class. Representative examples contained in the training set can be reviewed in Fig. 2.8.
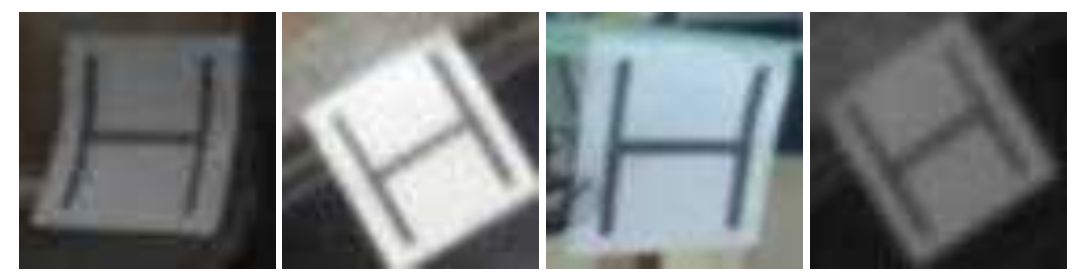

(a)
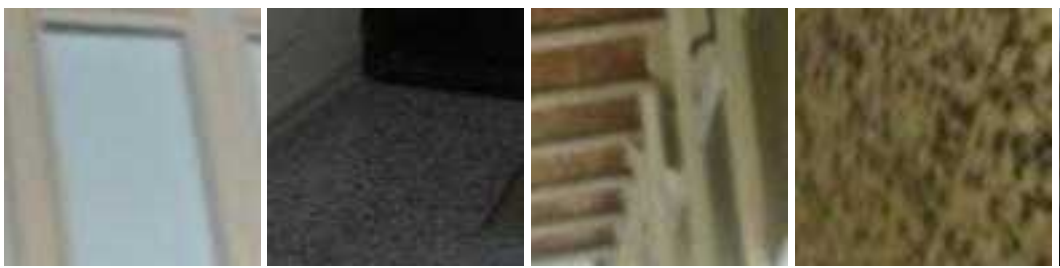
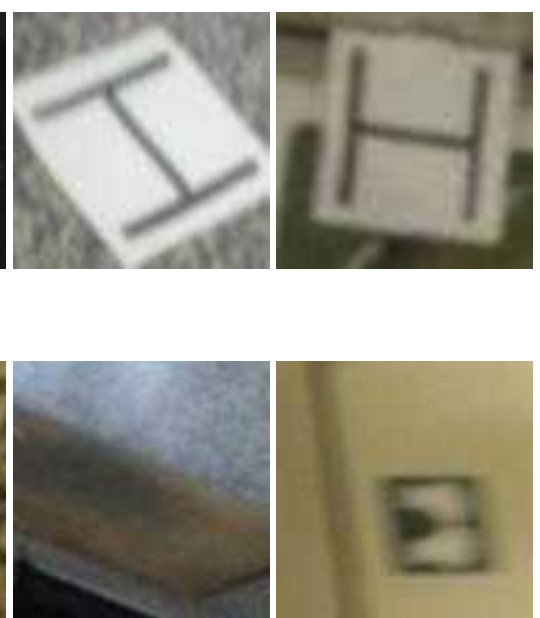

(b)

Figure 2.8: Examples of images used for training the helipad detection algorithms. (a) Helipad training examples. (b) Background training examples.

In order to evaluate the proposed helipad detection algorithm and compare it to the baseline methods, three test sequences containing indoor and outdoor scenes have been annotated at ROI-level. The first sequence containing a total of 1140 annotated frames has been captured by a human operator with the purpose of evaluating the performance of the different helipad detection methods for detecting a helipad in an unstructured indoor scenario. The second and third test sequences, containing 1749 and 1661 annotated frames respectively, have been captured during real flights. Thus, these sequences are more representative for the real conditions where the algorithms should operate.

\section{Training methodology}

In this section we present the methodology used for training the supervised learning classifiers designed for helipad detection purposes. Regarding the logistic regression classifier, the training methodology is based on a quadratic optimization formulation in which we add an L2 regularization term (see Eq. 2.3).

$$
\min _{\boldsymbol{\omega}} \frac{1}{2} \boldsymbol{\omega}^{T} \boldsymbol{\omega}+C \sum_{i=1}^{l} \log \left(1+e^{-y_{i} \boldsymbol{\omega}^{T} \mathbf{x}_{i}}\right)
$$

where $\boldsymbol{\omega}$ are the parameters to be learned by the classifier. $C$ is the regularization parameter, and $\left(\mathbf{x}_{i}, y_{i}\right), y_{i} \in\{-1,1\}$, is the instance-label pair of the $i_{t h}$ training sample. 
Similarly, the training methodology for the SVM classifier is based on a quadratic programming formulation which includes a regularization term and the classical restrictions of SVM classifiers based on the definition of slack variables (see Eq. 2.4).

$$
\begin{array}{ll}
\min _{\boldsymbol{\omega}, b, \boldsymbol{\xi}} & \frac{1}{2} \boldsymbol{\omega}^{T} \boldsymbol{\omega}+C \sum_{i=1}^{l} \xi_{i}, \\
\text { s.t. } & y_{i}\left(\boldsymbol{\omega}^{T} \phi\left(\mathbf{x}_{i}\right)+b\right) \geq 1-\xi_{i}, \quad \xi_{i} \geq 0
\end{array}
$$

where $\phi\left(\mathbf{x}_{i}\right)$ is a feature mapping function which allows to map the inputs to a higher dimensional space where a hyperplane defined by the parameters $\boldsymbol{\omega}$ (weights), $b$ (intercept term) can be applied in order to separate the instances into helipad or background classes. $C$ is the regularization parameter, $\left(\mathbf{x}_{i}, y_{i}\right), y_{i} \in\{-1,1\}$, is the instance-label pair of the $i_{t h}$ training sample, and $\xi_{i}$ are the slack variables which model the margin violation.

Taking into account the formulation presented in Eq. 2.3 and Eq. 2.4, the training process of the L2R-LR and the SVM with linear kernel has been performed by conducting a grid search over the regularization parameter $(C)$ using a 5-fold cross-validation procedure over the training set. The results obtained in this training process are shown in Fig. 2.9.

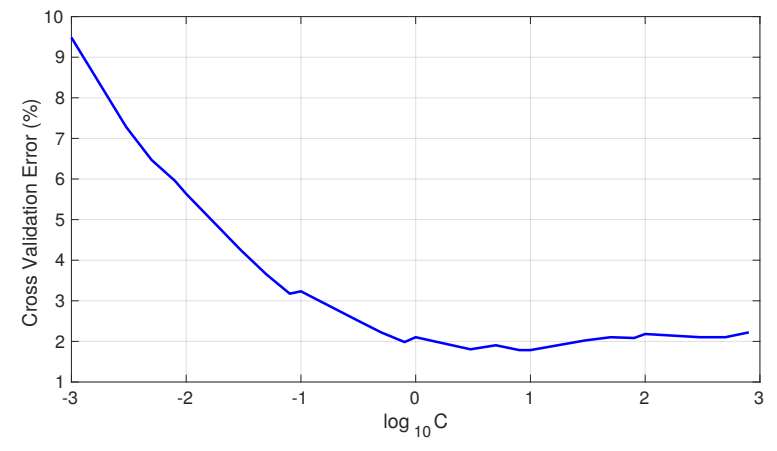

(a)

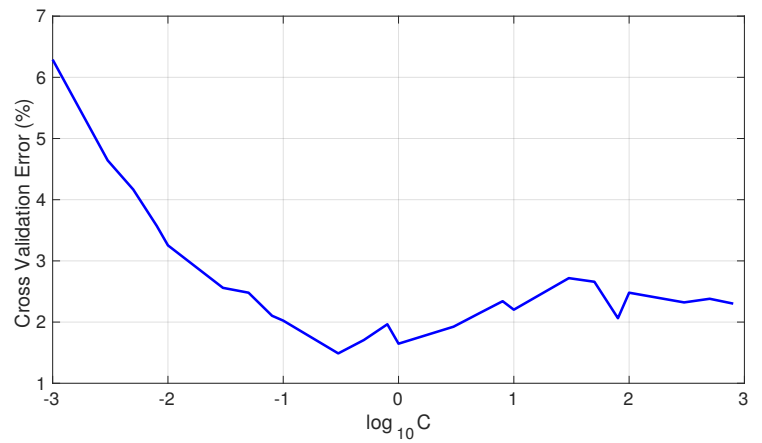

(b)

Figure 2.9: Results of the 5-fold cross-validation procedure for selecting the optimal parameters of the supervised learning classifiers used for helipad detection. a) Results obtained for the L2RLR classifier $\left(C_{o p t}=8\right)$. b) Results obtained for the L-SVM classifier $\left(C_{o p t}=0.3\right)$.

\section{Evaluation metrics and baseline methods}

In order to evaluate the helipad detection capabilities, we use the metrics utilized for tower detection purposes (see Section 2.5.2). That is, we take into account the mean Intersection over Union (mean IoU) which provides an estimation of the overlap between two bounding boxes. Furthermore, in contrast to the problem of tower detection, where the proposed model does not provide a probability of an object being a tower (this is because the proposed tower detection operates at a pixel level), the proposed algorithms for helipad detection provide a probability of belonging to the helipad class. Taking into account this output, we use the mean Average Precision $(\mathrm{mAP})^{6}$, which makes use of the

\footnotetext{
${ }^{6}$ https://github.com/Cartucho/mAP
} 
IoU greater than a threshold (in this case we use a threshold of 0.5) for considering the corresponding detection as a True Positive or False Negative (as compared to the ground truth ROI). Then, the area under the Precision-Recall curve is used to provide the final mAP measurement.

Regarding the baseline methods, we use the same state-of-the-art algorithms utilized in the evaluation of the tower detection algorithms. That is, in this case we consider the YOLOv2 and YOLOv3 for helipad detection purposes. In this case, only one image resolution $(736 \times 576$ pixels $)$ has been utilized for training the baseline algorithms, since when using an image resolution of $256 \times 256$ pixels the training process was not converging correctly.

\section{Results in helipad detection}

The results obtained after the evaluation of the proposed methods for helipad detection in the three test video sequences are summarized in Table 2.4, and shown in Fig. 2.10 for the best performing models in each sequence.

Table 2.4: Results obtained in the evaluation of the proposed method for helipad detection. Best results are highlighted in bold. The processing time of the L2R-LR and L-SVM is measured on an Intel Core i7-6700HQ CPU, whereas the one of the YOLO-based methods is computed on an Nvidia GeForce GTX 1080Ti GPU.

\begin{tabular}{cccccc}
\hline Sequence & \#frames & Method & mean IoU $(\%)$ & mAP $(\%)$ & Avg. FPS \\
\hline \multirow{4}{*}{ Seq1 } & \multirow{3}{*}{1140} & HOG + L2R-LR & $\mathbf{5 7 . 9 5}$ & $\mathbf{6 5 . 3 4}$ & $\mathbf{3 6 . 7 0}$ \\
& & HOG + L-SVM & $\mathbf{6 0 . 4 3}$ & $\mathbf{6 8 . 8 1}$ & 32.50 \\
& & YOLOv2 & 49.99 & 57.93 & $\mathbf{4 7 . 4 4}$ \\
& & YOLOv3 & 42.46 & 42.73 & 29.67 \\
\cline { 3 - 6 } Seq2 & \multirow{2}{*}{1749} & HOG + L2R-LR & $\mathbf{7 8 . 6 3}$ & 91.60 & $\mathbf{3 5 . 1 4}$ \\
& & HOG + L-SVM & $\mathbf{8 1 . 5 5}$ & $\mathbf{9 5 . 0 3}$ & 29.72 \\
& & YOLOv2 & 67.61 & 91.20 & $\mathbf{4 6 . 8 3}$ \\
& & YOLOv3 & 72.73 & $\mathbf{9 7 . 5 9}$ & 30.10 \\
\cline { 3 - 6 } Seq3 & \multirow{4}{*}{1661} & HOG + L2R-LR & $\mathbf{8 5 . 2 0}$ & $\mathbf{9 6 . 8 7}$ & $\mathbf{3 6 . 2 1}$ \\
& & HOG + L-SVM & $\mathbf{8 6 . 3 8}$ & $\mathbf{9 8 . 1 9}$ & 30.95 \\
& & YOLOv2 & 74.42 & 94.70 & $\mathbf{4 6 . 8 7}$ \\
& & YOLOv3 & 78.27 & 95.67 & 31.12 \\
\hline
\end{tabular}

The results depicted in Table 2.4 reveal the outstanding helipad detection capabilities of the proposed algorithms based on the combination of a shape-based region proposal, a HOG feature extractor, and a supervised learning classifier (i.e., logistic regression, and SVM with linear kernel). More concretely, the proposed algorithm based on the use of LSVM provides the highest mean IoU in all the sequences and the highest mAP in the first and third sequences. Similarly, the proposed algorithm based on the L2R-LR classifier provides the second-best result, being only surpassed in mAP by the YOLOv3 method in 


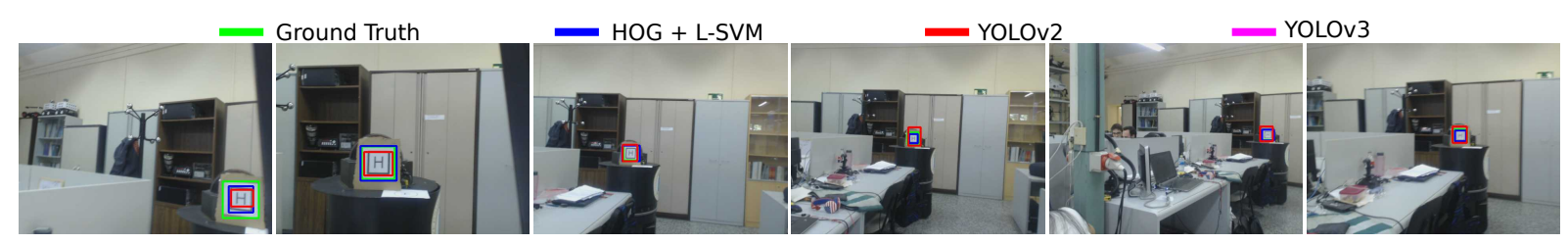

(a)

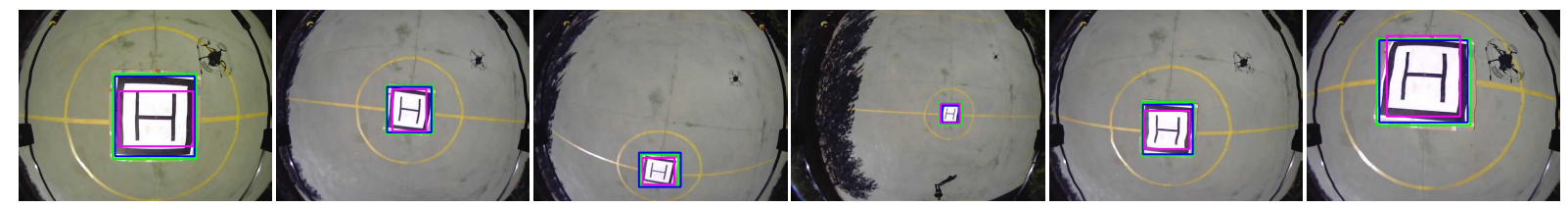

(b)
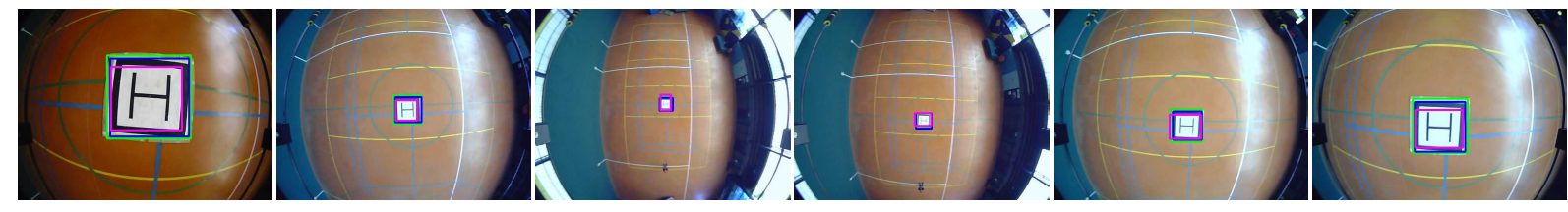

(c)

Figure 2.10: Helipad detection results obtained in several test sequences captured during indoor and outdoor experiments. (a) Detection results obtained in sequence 1 captured in an indoor scenario. (b) Results obtained in sequence 2 for an outdoor flight. (c) Results obtained in sequence 3 during an indoor flight.

the second test sequence. Taking into account the computational cost, YOLOv2 provides the highest FPS rate. However, it should be noted that this metric has been obtained on an Nvidia GeForce GTX 1080Ti GPU. In contrast, the proposed methods have been evaluated on an Intel Core i7-6700HQ CPU. Thus, the average FPS rate provided by the HOG + L2R-LR algorithm (36 FPS considering the three video sequences) reveals the real-time inference capabilities of this algorithm, which represents an appropriate candidate for its operation in platforms with limited computational capabilities.

Finally, the whole algorithm for helipad detection (i.e., including the pose estimation stage) is evaluated in the two sequences involving real flights (i.e., sequence 2 and 3 in Fig. 2.10). The final supervised learning classifier selected for helipad detection is the L-SVM classifier, which is the one showing the best average performance across the three sequences of Table 2.4. The detection provided by this classifier is used by the pose estimation module (see Section 2.3) in order to infer the pose of the UAV's bottom camera relative to the helipad. In this regard, we evaluate the pose estimation stage of our proposed algorithm in the two sequences involving real flights (i.e., sequence 2 and 3 in Fig. 2.10). More concretely, we evaluate the estimation in altitude since the only source of ground truth for the sake of evaluation is provided by the laser altimeter. The results obtained using the proposed helipad detection algorithm are depicted in Fig. 2.11.

The results obtained in Fig. 2.11 reveal the outstanding altitude estimation capabilities of the proposed method based on a $\mathrm{P} n \mathrm{P}$ algorithm with RANSAC for outliers rejection. Using only monocular information and the proposed algorithm, the Mean Absolute Errors 


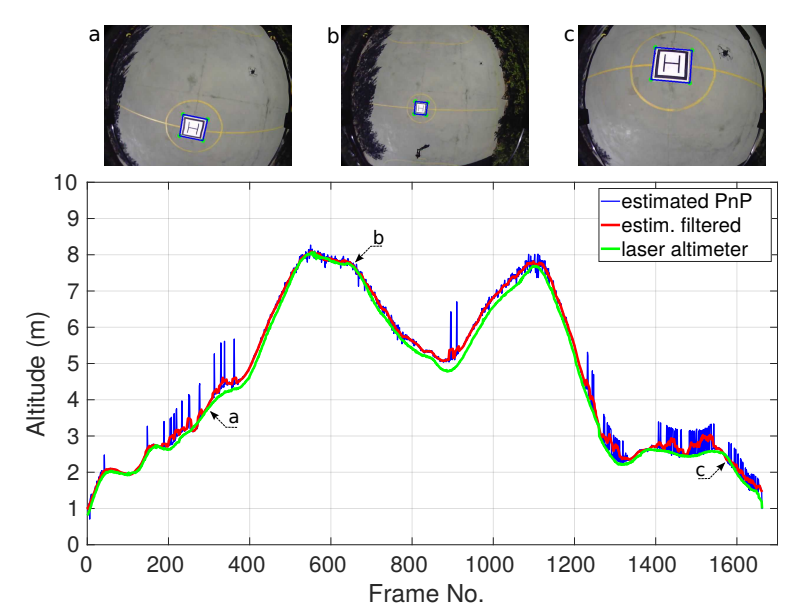

(a)

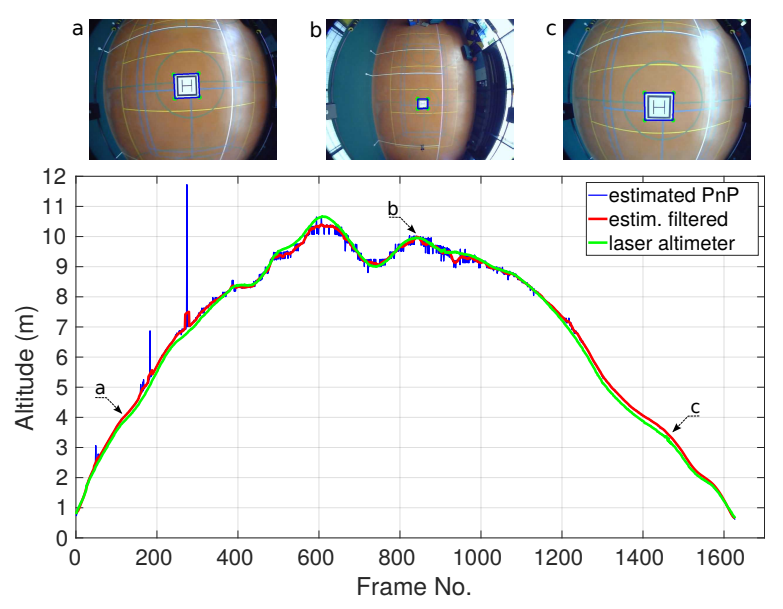

(b)

Figure 2.11: UAV altitude estimation using the helipad detection results obtained in the sequences concerning real-flight experiments. (a) Altitude estimation and helipad detection results obtained in sequence 2. (b) Altitude estimation and helipad detection results obtained in sequence 3. The estimated altitude of the UAV is computed using a $\mathrm{P} n \mathrm{P}$ algorithm. Laser measurements are provided by a Lightware SF10/A altimeter.

(MAE) obtained for sequence 2 and 3 are $0.187 \mathrm{~m}$, and $0.137 \mathrm{~m}$, respectively. These results are evidenced in Fig. 2.11 where it can be observed the almost exact match between the filtered estimation of the proposed algorithm (see the red curve in Fig. 2.11a and 2.11b) and the altitude provided by the laser altimeter (Lightware SF10/A). The coincidence between both altitude measurements is only perturbed in some moments of the flight where the detection jumps from a larger to smaller ROI and vice versa (see blue curve in frames 200 to 380 and 1400 to 1600 in Fig. 2.11a). These jumps are mainly due to the Canny edge detector and the posterior stage for finding contours, which only considers as valid contours for the subsequent region proposals stage the ones with a closed contour. In order to filter these oscillations in the estimated altitude caused by jumps in the detection, a moving average filter is applied over the last 10 estimated altitude measurements.

\subsection{Discussion}

The results presented throughout this chapter reveal the versatility exhibited by the proposed Fully Convolutional Network (Up-Net). The Up-Net network was initially designed for insulator string segmentation, as described in Sampedro et al., 2019, and has been adapted in this chapter for electric tower detection purposes. Furthermore, regarding the second use case analyzed in this chapter (i.e., helipad detection), the reported results show the outstanding detection capabilities (in terms of accuracy and computational cost) exhibited by the proposed helipad detection method.

The results obtained in medium voltage electric tower detection, shown in Table 2.1. are the result of an evaluation procedure over 6491 annotated frames. The results ob- 
tained in Table 2.1 reveal that YOLOv3 is the algorithm with the highest performance, achieving an average mean IoU of $83.7 \%$ when training the algorithm at the resolution of $256 \times 256$ pixels and considering the weighted average over the number of frames of both test sequences. This corresponds to an increment in mean IoU of $2.2 \%$ as compared to YOLOv2 trained at the same resolution, which in contrast allows an increment of $\times 1.4$ in the FPS rate. Similar results to the ones obtained by YOLOv3 are achieved by the proposed Up-Net_vgg16_aug, which gets an average mean IoU of $83 \%$. This result is benefited by the application of data augmentation techniques (i.e., horizontal flip), which provide an improvement of $1.8 \%$ in the average mean IoU as compared to the original model (Up-Net_vgg16). The Up-Net variant proposed in this chapter, named Up-Net_tiny, allows reducing the number of parameters by a factor of $\times 16$ (from 34.45 $\mathrm{M}$ to $2.22 \mathrm{M}$ ), what allows to increment the FPS rate by a factor $\times 4.5$ (from $47 \mathrm{FPS}$ to 215.1 FPS). Despite this drastic reduction in the number of parameters, the proposed Up-Net_tiny, when trained using the data augmentation techniques described previously (Up-Net_tiny_aug) achieves an average mean IoU of $80.4 \%$ as shown in Table 2.1, which represents a reduction of only $2.6 \%$ as compared to the Up-Net_vgg 16 _aug. The performance of the proposed Up-Net_tiny_aug is also benefited by the application of data augmentation techniques, allowing an improvement of $2.6 \%$ in the average mean IoU.

The results obtained in high voltage electric tower detection, shown in Table 2.2, correspond to an evaluation procedure over 2806 annotated frames. These results reveal the outstanding detection capabilities of the proposed Up-Net_vgg16_aug, which provides the best performance, with an average mean IoU of $85.6 \%$, considering the weighted average over the number of frames of both test sequences. As in the case of the medium voltage, this performance is benefited by the application of data augmentation techniques, which in this case allow an increment of $3.9 \%$ with respect to the base model (Up-Net_vgg16). A similar increment in performance of $3.6 \%$, when training using the augmented dataset, is exhibited by the proposed Up-Net_tiny_aug as compared to its counterpart trained in the original dataset (Up-Net_tiny). In the case of the high voltage tower detection problem, the proposed Up-Net_tiny_aug achieves an average mean IoU of $80.4 \%$, which corresponds to a reduction of $5.2 \%$ as compared to the Up-Net_vgg16_aug. However, as in the case of the medium voltage problem, the tiny version of the proposed FCN allows an important increment factor in the FPS rate, which according to Table 2.2 is $\times 4.1$ (from 47.3 FPS to 196.2 FPS). This result makes the proposed Up-Net_tiny an appropriate candidate for being integrated into systems with severe computational constraints. Regarding the YOLO-based detectors, the YOLOv3 achieves an average mean IoU of $82.6 \%$ when trained considering an original resolution of $256 \times 256$ pixels. This corresponds to an increment of $3.2 \%$ with respect to the YOLOv2, which obtains an average mean IoU of $79.4 \%$. As in the case of the analysis performed for medium voltage, regarding the FPS rate, the YOLOv2 provides an increment of $\times 1.3$ as compared to YOLOv3.

Regarding insulator string segmentation, throughout the results presented in Section 2.5.3, it is shown that semantic segmentation techniques are able to provide accurate segmentation results for the insulator recognition task without the need for a prior stage using an object detection algorithm. Avoiding a previous stage of object detection can lead to considerable savings in computational resources, which can become of utmost 
importance in systems with hard computational constraints. In this work, we have conducted an extensive evaluation and comparison of several semantic segmentation techniques, ranging from classical state-of-the-art models to more sophisticated approaches involving GANs. The proposed architecture for semantic segmentation, termed Up-Net, has demonstrated outstanding capabilities obtaining a mean accuracy and mean IoU of $95.16 \%$ and $92.06 \%$, respectively. Furthermore, we have explored the integration of the proposed Up-Net within a GAN configuration (GAN_Up-Net). The results obtained when using the GAN_Up-Net correspond to the second best performing model in terms of pixel accuracy, mean accuracy and frequency weighted IoU $(98.24 \%, 94.60 \%$, and $96.73 \%$ respectively), which consolidates the proposed architecture.

The modifications added to the base U-Net network provide an improvement with respect to the original network of $1.2 \%, 1.1 \%$, and $0.7 \%$ in mean accuracy, mean IoU, and frequency weighted IoU respectively when training in the final dataset utilized (augmented dataset). These results reveal how the semantic information coming from deeper layers can be efficiently incorporated by the new skip connections introduced in the Up-Net architecture, refining the segmentation results.

Results presented in Table 2.3 also reveal the influence that the data augmentation and transfer learning techniques have in the insulator string segmentation task. The former consists of using a horizontal flip and two gamma correction operations which allow multiplying by 4 the number of training images in the original dataset. Transfer learning is explored in this chapter by using the VGG16 network pre-trained on the ImageNet dataset. The analysis of the results presented in Table 2.3 shows the positive impact of the data augmentation and transfer learning techniques on the performance of the semantic segmentation networks evaluated in this work. In most of the networks, the usage of the proposed data augmentation strategy produces an increment between $2 \%$ to $5 \%$ and $3 \%$ to $7 \%$ in mean accuracy and mean IoU, respectively. The networks more influenced by the data augmentation strategy are the FCN-8s with an improvement in mean accuracy and mean IoU of $4.7 \%$ and $6.3 \%$ respectively, and the GAN_U-Net which improves the mean accuracy and mean IoU by $4.3 \%$ and $7.3 \%$ respectively. Using transfer learning, most of the networks experience an increment in mean accuracy and mean IoU between $4 \%$ to $11 \%$ and $4 \%$ to $12 \%$ respectively, being the SegNet network the one which exhibits highest improvements with an increment in mean accuracy and mean IoU of $10.5 \%$ and $12.3 \%$, respectively.

Concerning the results obtained for helipad detection, in this analysis, we proceed as in the case of the analysis conducted for electric tower detection by computing the weighted average taking into account the number of frames of the sequences presented in Table 2.4. Moreover, we analyze separately the results obtained in the first sequence, which was captured by a human operator in an indoor scenario, and on the other hand, we analyze the combined results in sequences 2 and 3, which were captured during real-flight conditions. In this regard, the results obtained in the first sequence (seq1) reveal the good detection performance of the proposed methods as compared to the baseline algorithms. The best performing method in the first sequence is the HOG+L-SVM method, which obtains a mean IoU of $60.4 \%$ and a mAP of $68.8 \%$. These results outperform the HOG+L2R-LR, YOLOv2, and YOLOv3 in 2.5\%, 10.4\%, and 18\% respectively when considering the mean 
IoU, and in $3.5 \%, 10.9 \%$, and $26.1 \%$ respectively when considering the mAP. It should be noted that the results obtained for the first sequence are in a certain way not very high since at certain instants of this sequence the helipad presents a very small size in the image (see last image in Fig. 2.10a). This fact hinders the detection capabilities of all the detection methods analyzed, and represents an especial challenge for YOLOv3, which obtains a mean IoU of $42.46 \%$, and a $\mathrm{mAP}$ of $42.73 \%$. This result is counterintuitive in the sense that one of the improvements introduced in YOLOv3 is the detection at three levels of the feature pyramid created by this model, and can be due to the anchors defined for this model, which have been set with the default values during training. This effect will be further analyzed in future works.

With respect to the helipad detection results in the second and third sequences, the results presented in Table 2.4 have a similar tendency as the ones obtained for the first sequence, with the difference that in these sequences, the method which obtains the highest performance in terms of the average mAP is the YOLOv3. This model achieves an average mAP of $96.7 \%$, which is only $0.1 \%$ greater than the one obtained by the $\mathrm{HOG}+\mathrm{L}-\mathrm{SVM}$ (96.6\%). The latter outperforms the HOG+L2R-LR, YOLOv2, and YOLOv3 in 2.1\%, $13 \%$, and $8.1 \%$ respectively when considering the average mean IoU, and outperforms the $\mathrm{HOG}+\mathrm{L} 2 \mathrm{R}-\mathrm{LR}$, and YOLOv2 in $2.4 \%$, and $3.7 \%$ respectively when analyzing the average mAP. With respect to the computational cost, it should be noted that the results presented in Table 2.4 for the $\mathrm{HOG}+\mathrm{L}-\mathrm{SVM}$ and $\mathrm{HOG}+\mathrm{L} 2 \mathrm{R}-\mathrm{LR}$ have been measured on an Intel Core i7-6700HQ CPU. The proposed methods obtain an average FPS rate of 35.7 and 30.3 for the $\mathrm{HOG}+\mathrm{L} 2 \mathrm{R}-\mathrm{LR}$ and the $\mathrm{HOG}+\mathrm{L}-\mathrm{SVM}$, respectively. These results provide an intuition of the less demanding computational capabilities of the proposed methods as compared to the baselines, taking into account the average results obtained for YOLOv2 (46.8 FPS) and for YOLOv3 (30.6 FPS) which are measured on an Nvidia GeForce GTX 1080Ti GPU. Finally, the complete system integrating the best performing supervised learning classifier (L-SVM) is evaluated for altitude estimation purposes. The evaluation results obtained are shown in Fig. 2.11, and reveal very low altitude estimation errors as compared to the measurements provided by the laser altimeter (MAE of $0.19 \mathrm{~m}$ and $0.14 \mathrm{~m}$ across the whole flight of sequences 2 and 3, respectively). These accurate results in altitude estimation are benefited by the shaped-based region proposal algorithm integrated into our system. As compared to other type of detectors, the one proposed in this chapter works directly on the contour of the object, which is crucial for obtaining accurate pose estimation results. On the other hand, grid-based or sliding window detectors, might provide detections in which the ROI can be slightly away from the object contour. These detections can intuitively lead to inaccurate pose estimation results. The results presented in Fig. 2.11, demonstrate the effectiveness of monocular approaches for pose estimation purposes, which can be an appropriate solution in systems with limited payload capabilities (e.g., MAVs) where laser altimeters cannot be integrated.

Overall, the results obtained for helipad detection reveal the outstanding detection capabilities of the proposed methods based on the combination of a region proposal stage (based on shape information), a feature extraction stage based on HOG, and a fast supervised learning classifier (i.e., logistic regression, and SVM with a linear kernel). This shows that in some particular problems, where the shape of the object to be detected is 
well-defined, simpler methods, such as the ones proposed here, can boost sophisticated state-of-the-art techniques such as YOLOv2 and YOLOv3. Furthermore, the analysis on computational cost conducted throughout the experiments reveals that the proposed methods can get accurate detections in real-time on a standard CPU and thus are good candidates for their integration into systems with limited computational capabilities.

\subsection{Conclusions}

In this chapter, two different algorithms of varying nature have been proposed for solving two main uses cases. Electric tower detection and insulator string segmentation have been solved with the objective of applying posterior diagnosis stages. On the other hand, accurate helipad detection and altitude estimation have been proposed in this chapter as a prior stage for performing autonomous landing maneuvers on moving platforms.

Regarding the electric tower detection and insulator string segmentation, a novel Fully Convolutional Network, termed Up-Net, has been proposed in this chapter. The proposed Up-Net is an adaptation of the state-of-the-art U-Net network, which is modified in this work by enlarging the encoder network to a VGG16 architecture, and adding new skip connections at certain levels of the architecture. These connections have shown to be beneficial in order to further incorporate high-level semantic information from deeper layers. Furthermore, for electric tower detection a reduced version of the proposed architecture, named Up-Net_tiny, has been designed which allows reducing by a factor of $\times 16$ the number of parameters in the network, with a consequent increment in the FPS rate of approximately $\times 4.5$ (measured on an Nvidia GeForce GTX 1080Ti GPU). The same UpNet architecture has been trained in a semantic segmentation fashion for solving both problems of electric tower detection and insulator string segmentation. A thorough evaluation of the proposed architecture for electric tower detection in medium and high voltage power lines has been conducted. The evaluation for medium voltage tower detection has been conducted using two sequences of real aerial inspections encompassing 6491 frames. The results obtained after the evaluation show that the proposed Up-Net, when pretrained using the weights of the VGG16 (transferred to the encoder network) and trained in the augmented dataset (Up-Net_vgg16_aug) is able to achieve state-of-the-art detection results providing a mean IoU of $83 \%$ considering both medium voltage sequences. This performance is almost identical to the one provided by the YOLOv3 baseline (83.7\%), what reveal the outstanding capabilities of the proposed network. Similarly, an exhaustive evaluation of the proposed Up-Net has been conducted for high voltage tower detection. In this regard, two sequences of real flight inspections encompassing 2806 frames have been used. The results obtained after the evaluation reveal a similar conclusion as the one extracted for the medium voltage case. The proposed Up-Net_vgg16_aug achieves a mean IoU of $85.6 \%$ taking into account the weighted average (based on the number of frames) for both high voltage sequences. This performance outperforms the one obtained by the best baseline, YOLOv3, which achieves a mean IoU of $82.6 \%$. The tiny version of the proposed Up-Net, when trained on the augmented dataset provides accurate detection results (mean IoU of $80.4 \%$ in both medium and high voltage sequences), which is close to state-of-the-art performance. At the same time, this network provides much than 
real-time detection capabilities $(\approx 215$ FPS $)$, which makes it suitable for platforms with limited computational resources.

Regarding the insulator string segmentation, the proposed Up-Net, and its variant GAN_Up-Net (when training Up-Net within a Generative Adversarial Network framework) have shown accurate segmentation capabilities for segmenting the different elements in the insulator string (i.e., caps and discs) under a wide range of conditions with varying insulator type (e.g., ceramic and glass), lighting conditions, point of view and orientation of the insulator string, among others. These accurate segmentation capabilities facilitate the posterior stages for fault diagnosis. The proposed Up-Net_vgg16 when trained on the augmented dataset achieves a mean accuracy and mean Io $\bar{U}$ of $95.16 \%$ and $92.06 \%$, respectively, which represents the best performance as compared to several state-of-theart semantic segmentation networks. These results are positively influenced by the use of data augmentation (i.e., horizontal flip, and two gamma correction operations) and transfer learning techniques (using the VGG16 trained on ImageNet) which allow increasing by a large margin the performance of the proposed model and state-of-the-art networks. Furthermore, the Up-Net_vgg16 provides an inference time of $19.3 \mathrm{~ms} /$ image (measured on an Nvidia GeForce GTX 1080Ti GPU), revealing real time capabilities.

Finally, the proposed algorithm for helipad detection, based on the combination of a shape-based region proposal algorithm, a feature extraction stage based on HOG features, a fast supervised learning classifier (i.e., L2 regularized logistic regression, and SVM with linear kernel), and a $\mathrm{P} n \mathrm{P}$-based pose estimation method, have shown to provide accurate helipad detection and altitude estimation capabilities as compared to state-of-the-art object detectors. In this regard, the evaluation of the proposed methods for helipad detection has been conducted using two types of image sequences, one captured by a human operator encompassing 1140 annotated frames, and two sequences captured during real flights, containing 3410 annotated frames. The results obtained in these sequences reveal the outstanding detection capabilities of the proposed HOG + L-SVM which achieves the best detection results in the first sequence, with a mean IoU of $60.43 \%$ and a mAP of $68.81 \%$, and the best average mean IoU $(83.9 \%)$ in the second and third sequences. Furthermore, the proposed HOG $+\mathrm{L}-\mathrm{SVM}$ method is the second best performing model in terms of mAP, obtaining an average $\mathrm{mAP}$ of $96.6 \%$, which is just $0.1 \%$ lower than the one obtained by the YOLOv3 baseline (96.7\%). These results confirm that in some problems where the shape of the object is well-defined, classic algorithms for object detection can be an appropriate alternative to more sophisticated state-of-the-art algorithms. Furthermore, the proposed $\mathrm{HOG}+\mathrm{L}-\mathrm{SVM}$ obtains an average rate of $30.3 \mathrm{FPS}$ (measured on an Intel Core i7-6700HQ CPU) in the second and third sequences, revealing real-time capabilities. On the other hand, the very low MAE $(0.19 \mathrm{~m}$ and $0.14 \mathrm{~m})$ in the two real-flight experiments reveal the appropriate capabilities of monocular approaches for altitude estimation purposes. The $\mathrm{P} n \mathrm{P}$-based pose estimation stage integrated into our system has proven to be an effective solution for altitude estimation, which can be used in aerial robots with limited payload, such as MAVs, as an alternative to laser altimeters. Furthermore, in these platforms, the altitude estimation provided by the proposed approach can be fused with the altitude measurements provided by other sensors (e.g., barometer) in order to refine the estimation. 
Overall, it should be emphasized that:

- Fully Convolutional Networks provide an excellent framework for object detection and semantic segmentation not only in accuracy but also in efficiency. When these types of networks include skip connections in their architectures, such as the proposed Up-Net or the YOLOv3 network, a semantically rich Feature Pyramid can be generated, which greatly benefits the accuracy of the network.

- Additional deep learning techniques such as data augmentation and transfer learning have proven to benefit the performance of most of the networks used in this chapter for object detection and semantic segmentation. This fact opens an interesting future research topic.

- When the shape of the object to be detected is well-defined or can be easily modeled owing to its simplicity (e.g., a helipad object), the exploration of more classic object detectors might be worth. The proposed methods for helipad detection which combine a shape-based region proposal, with a HOG feature extractor and a fast supervised learning classifier, have shown accurate and real-time detection capabilities. The integration of the proposed method for helipad detection into our current system for autonomous landing on moving platforms using reinforcement learning (Rodriguez-Ramos et al., 2018), has an almost direct transition since the latter is based on the relative Euclidean distance between the UAV and the helipad. This integration will lead to a complete autonomous landing system, whose implementation will be considered for future works. 



\section{Chapter 3}

\section{Anomaly Detection}

\section{$3.1 \quad$ Introduction}

In this chapter, we present different supervised and semi-supervised learning solutions for detecting anomalies or faults in one of the most important elements that comprise the electric power transmission lines, such as insulator strings. Furthermore, we integrate the proposed diagnosis components with the insulator string segmentation component described in Section 2.4 to build a complete and fully automatic system for power transmission line inspection and diagnosis.

Anomalies, also named outliers, are patterns in data that do not fit into the notion of a normal pattern or representation of data (Chandola et al., 2009). In this thesis, we define the concept of anomaly as an image sample which presents abnormal regions of pixels in the image (referred to as a fault) as compared to normal image samples. Leveraging the results presented in the previous chapter for the use case of power line inspection, the objective of the algorithms presented in this chapter is to develop a fully automatic fault diagnosis system, capable of detecting the anomalies or faults that can occur in the power transmission line elements (e.g., insulator strings).

As stated in Chandola et al., 2009, based on the availability of training data regarding the normal and abnormal class, the anomaly detection techniques can be classified into three main categories:

- Supervised anomaly detection: In this category, the learning algorithm is designed as a standard discriminative model which has to distinguish between the normal and anomalous classes. The most significant problem in this case is the availability of training data regarding the anomaly class. In most anomaly detection problems, the abnormal examples are usually rare and difficult to obtain. Thus, several techniques for dealing with imbalanced datasets have emerged (Wang et al., 2016; Buitrago et al., 2018). Other techniques address the problem of obtaining representative training examples of anomalies by injecting artificially generated anomalies into normal examples (Abe et al., 2006).

- Semi-supervised anomaly detection: This mode of learning assumes that only training data relative to the normal class are available. These learning techniques are 
more widely applicable than the pure supervised approach since they do not require training examples of the anomaly class. Thus, the acquisition of the training data is limited to collecting samples of the normal class, which simplifies the implementation of these approaches.

- Unsupervised anomaly detection: Techniques that fall within this category make use of unlabeled data under the assumption that normal examples occur much more frequently than abnormal ones.

In this chapter, we explore the anomaly detection problem within the context of supervised and semi-supervised learning techniques. For this purpose, within the supervised learning framework, we propose a novel method which integrates Siamese Convolutional Neural Networks (SCNNs) for diagnosing several types of defects that might occur in the insulator string elements (e.g., discs polluted, burned, rusted, etc.). The proposed SCNNs are used for modeling the similarity between input images (e.g., discs within an insulator string), and we demonstrate that they represent an effective solution for fault detection purposes. Furthermore, traditional classifiers such as CNNs are also used as the main techniques for recognizing one of the most critical anomalies in insulator strings related to the absence of disc units, and are also utilized as a baseline for evaluating the performance of the SCNNs. In order to deal with the small number of training samples related to the anomaly class, we propose a solution similar to other state-of-the-art works (Abe et al., 2006) that consists of creating synthetic defects that are introduced into healthy examples, intending to increase the number of defective samples for training in a supervised fashion. These solutions are explained in detail in Section 3.4.1. Regarding the semi-supervised learning framework, we propose a solution based on Siamese Convolutional Autoencoders (SCAE) and semi-supervised learning classifiers. The Autoencoders are utilized for unsupervised feature extraction of the normal data, allowing to obtain encodings which are subsequently used for training semi-supervised classifiers such as the One-Class SVM (OC-SVM) (Schölkopf et al., 2001) classifier. The solutions proposed using the semi-supervised learning framework are explained in Section 3.4.2.

As stated previously, in this chapter we present the solutions developed for anomaly or fault detection within the use case of power transmission line inspection. More concretely, we address the problem of automating the fault detection process in elements of the power transmission lines, such as insulator strings. Building such an automatic system requires robust insulator detection and diagnosis algorithms capable of dealing with the enormous variability in the captured data. In this context, images captured from helicopters, UAVs, or other platforms used for power line inspection, usually have very complex and heterogeneous backgrounds. In addition, insulators can be made of different materials (e.g., glass, ceramic, composite, etc.) which drives to a huge variability of the appearance of the insulator in the image. Glass and ceramic insulators surfaces can have reflections due to sunshine, which greatly hinder the diagnosis of possible defects. Furthermore, depending on the platform used to capture the images, insulators can appear with very different points of view on the image and even present occlusions, which significantly complicate the tasks of inspection and diagnosis. 
Added to the above difficulties is the enormous variability of defects that can appear in the insulator strings, varying from burned, rusted, polluted, or cracked elements to the presence of bird excrements on their surface. The latter has proven to be an important cause in the appearance of defects in electrical power supply facilities, such as flashovers (Taklaja et al., 2013). Furthermore, some defects are rare and only appear under particular conditions. Thus, there is a significant difficulty in obtaining enough data for each type of defect in all its variants. Under these constraints, where only a small number of examples per defect type are available, and when only some types of defects are known in advance at the time of training, traditional classification methods have some limitations as highlighted in Chopra et al., 2005. In these situations, siamese neural networks have shown to be an effective solution, providing remarkable results in other fields of computer vision such as face verification (Chopra et al., 2005) and character recognition (Koch et al., 2015).

In order to precisely detect the defects and diagnose them under the aforementioned conditions, a versatile system with advanced capabilities is required. For this purpose, strategies based on traditional computer vision algorithms can provide appropriate results in structured images under controlled illumination and background conditions. However, as stated in Chang et al., 2018, most of these approaches are based on heuristics which require several assumptions and handcrafted thresholds (Zhai et al., 2018, Han et al., 2018), which need to be manually tuned and re-adjusted to properly operate under previously unseen conditions. In contrast, strategies based on machine learning techniques, when trained in meaningful datasets, can provide more flexible solutions, making the fault diagnosis process more robust to changes in illumination, background, type of insulator, etc. As a drawback, machine learning models and especially deep learning ones, usually require a large amount of data to be trained properly.

Owing to enormous variability of elements disposed along the power transmission line infrastructure and the considerable variety of defect types that can appear within each element, the development of a completely automatic system for fault diagnosis purposes, that is, without the human intervention in the inspection process, is still far from being a reality. This means that in production systems, the presence of specialized human operators in the last stages of the inspection process is inevitable. For this purpose, in this chapter, we do not focus specifically on the accurate location of defects in the image but instead, we focus on providing accurate predictions of the frames that contain defective insulator strings within a video sequence. As a result, only the frames where our system has automatically found the insulator string to be defective will be further reviewed by a specialized human inspector, resulting in shortened inspection times.

\subsection{Related Work}

Anomaly detection in images has been mostly explored in the field of medical image processing (Seeböck et al., 2016, Schlegl et al., 2017; Alaverdyan et al., 2018), surface inspection tasks (Silva and Lucena, 2018; Kim and Cho, 2018; Minhas and Zelek, 2019), and remote sensing image classification (Muñoz-Marí et al., 2008; Muñoz-Marí et al., 2010 ). 
Most of the works that one can encounter in the literature for anomaly detection make use of OC-SVM (Muñoz-Marí et al., 2008; Muñoz-Marí et al., 2010; Seeböck et al., 2016; Erfani et al., 2016; Nguyen and Vien, 2018, Alaverdyan et al., 2018), which in most of the cases is trained using the encodings extracted from an autoencoder (Seeböck et al., 2016, Nguyen and Vien, 2018, Alaverdyan et al., 2018). In Seeböck et al., 2016, two deep Convolutional Autoencoders (CAEs) are used in combination with Denoising Autoencoders (DAEs) in order to extract feature representations of healthy data, which are then utilized for training an OC-SVM with linear kernel with the purpose of detecting anomalies in Optical Coherence Tomography (OCT) images. Similarly, in Nguyen and Vien, 2018 the authors propose deep autoencoders and CAEs which are trained conjointly with an OC-SVM (i.e., sharing the same loss function), in which the RBF kernel is approximated with Random Fourier Features. Closely related to the proposed approach presented in Section 3.4.2, is the work described in Alaverdyan et al., 2018, where a siamese neural network composed of stacked convolutional autoencoders as sub-networks are used as feature extractors for training an OC-SVM with the aim of detecting anomalies in brain images. Alternative solutions for anomaly detection in images make use of generative models such as Deep Belief Networks (DBN) or GANs. In Erfani et al., 2016, an OCSVM is trained using the features learned by the DBN, which acts as an unsupervised feature extractor. In Schlegl et al., 2017, a deep convolutional GAN is used to learn a manifold of normal anatomical variability, with the objective of identifying anomalies in OCT images.

Regarding the field of fault diagnosis in the electrical power transmission lines elements, most of the state-of-the-art works are based on supervised learning approaches and are mainly focused on the identification of absent disc units within the insulator string (Zhai et al., 2017; Zhai et al., 2018; Gao et al., 2017; Ling et al., 2018; Tao et al., 2018). Moreover, most of these approaches based their diagnosis capabilities on the computation of the distance (in pixels) between adjacent disc units (Zhai et al., 2017; Zhai et al., 2018, Gao et al., 2017), making them prone to unpredictable results when more than one disc is missing in the insulator string. More recent approaches make use of deep learning techniques for diagnosing the absent disc defect (Ling et al., 2018; Tao et al., 2018). In Ling et al., 2018 the U-Net network is used to segment the pixels corresponding to the region of the insulator string where the disc is absent. In the work proposed in Tao et al., 2018, the detection of the defect is performed at ROI-level using a Region Proposal Network (RPN) (Ren et al., 2015). In this work, we leverage the accurate segmentation results obtained by the proposed Up-Net described in Section 2.4 to build a CNN for the identification of absent disc units regardless of the number of disc units missing in the insulator string.

Despite the problem of absent disc units is very relevant due to its implications on possible interruptions of the electric power transmission system, other types of defects, such as contamination or pollution in insulator discs may also produce severe damages to the infrastructure such as flashovers (Taklaja et al., 2013). Only a few works in the literature address the identification of multiple defects. In Jabid and Ahsan, 2018, five types of defects were addressed by using a Nearest-Neighbor algorithm on handcrafted Local Directional Pattern (LDP) descriptors with Chi-square similarity measure. In the 
work presented in Zhao et al., 2016, authors addressed the diagnosis of cracked and dirty insulator discs using a CNN for feature extraction in combination with an SVM with RBF kernel. However, the results presented in these works were only validated in a few number of images of defective components. In contrast, we conduct an extensive evaluation of the proposed fault diagnosis system using several video sequences captured during real aerial inspections, each of them composed of thousands of frames.

Previous state-of-the-art approaches for detecting multiple types of defects use traditional supervised learning techniques under the assumption that there is no limitation in the acquisition of training data relative to normal and abnormal training examples. Thus, supervised learning techniques are applied in their standard fashion. However, as stated in Section 3.1. most frequent systems in anomaly detection have to deal with the problem of imbalanced datasets owing to the limited data relative to anomaly training examples. Taking these constraints into account, and inspired by the concepts proposed in Chopra et al., 2005, in this chapter we adopt an SCNN architecture which is responsible for computing the similarity between adjacent disc units with the purpose of diagnosing several types of disc defects using a unique model. A similar concept about measuring the similarity between adjacent disc units was also presented in Oberweger et al., 2014, with the difference that in our approach the weights of the SCNN are learned for maximizing the similarity/dissimilarity between pairs of non-defective or defective discs respectively, whereas in Oberweger et al., 2014 these descriptors were fixed and handcrafted.

\subsection{System Description}

In this section, we describe the proposed system for automatic detection and diagnosis of electrical insulator strings. The main objective of the proposed system is to automate the process of fault diagnosis in the RGB images captured after the inspection flights that are conducted on a regular basis by electric companies. For this purpose, the proposed system is based on the combination of different deep learning-based components for insulator string segmentation and fault diagnosis (see Fig. 3.1). The input to the system consists of an RGB image which is subsequently processed by the proposed Fully Convolutional Network (Up-Net) which acts as the core of the insulator string segmentation component explained in Section 2.4. As a result, a 2-channel mask is obtained where the different elements in the insulator string (i.e., caps and discs) are classified at pixel-level. Using the resulting mask, we compute the orientation of the insulator string and rectify it for further processing. This stage is performed by computing the orientation angle of the most prominent contour in the image associated with the segmented insulator string. Once the orientation angle is calculated, a warping operation consisting of a 2D rotation is applied to the pixels of the original RGB and segmentation mask images in order to rotate the insulator string vertically. Some examples of the output provided by this component can be reviewed in Fig 3.1 .

The rectified mask is used by the absent discs diagnosis component (see Section 3.4.1) in order to predict if the current frame contains a defective insulator string in these terms (i.e., absent disc units). At the same time, the rectified mask and input image are used to extract the Region Of Interest (ROI) of every element in the insulator string using 
image processing algorithms based on contours extraction and its subsequent filtering by area to remove small false positives that might arise in the insulator segmentation stage. Using these ROIs, the cropped images corresponding to the different discs are combined in pairs and passed to the SCNN, which works as the core of the damaged disc diagnosis component (see Section 3.4.1).

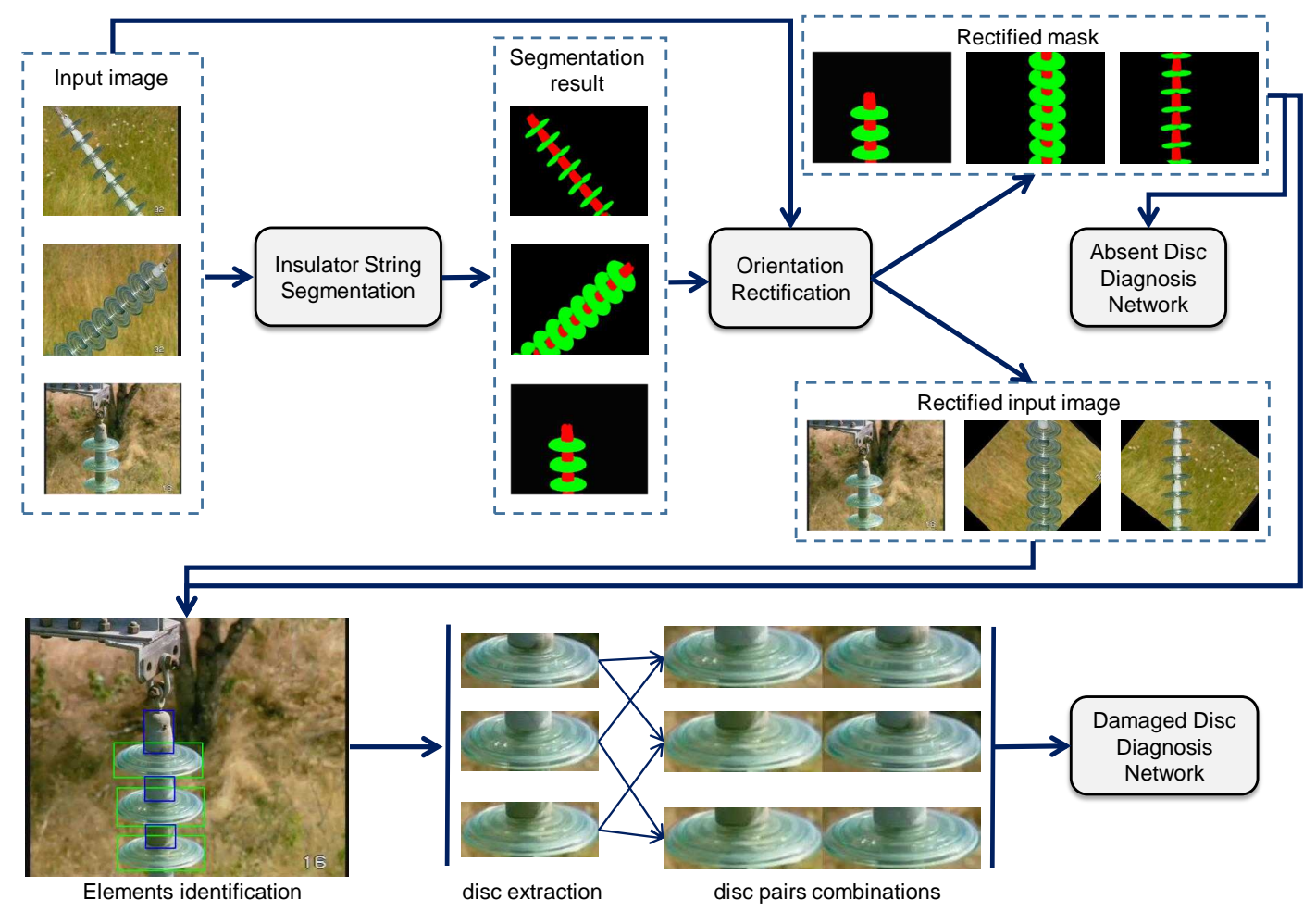

Figure 3.1: System description for the diagnosis of electrical insulator strings (best seen in color). The proposed system is mainly based on the combination of an insulator string segmentation component, a post-processing component for rectifying the orientation of the insulator string, and two components responsible for fault diagnosis.

\subsection{Learning-based Anomaly Detection}

\subsubsection{Supervised Anomaly Detection}

In this work, we divide the diagnosis of defects in the power line insulator strings into two separate problems. On the one hand, we leverage the output provided by the insulator string segmentation component described in Section 2.4 and use it as input to a CNN model trained for recognizing if there are disc units absent within the insulator string. On the other hand, we address the diagnosis of defects in the disc units of an insulator string by designing a siamese neural network which takes as input pairs of disc images extracted from the insulator string. 


\section{Diagnosis of absent disc units}

This component consists of a 10-layer CNN (see Fig. 3.2) which takes as input the segmentation mask (extended to 3 channels) provided by the insulator string segmentation component (see Section 2.4), and outputs a probability of the occurrence of the disc absence defect. Thus, the training procedure of this network has been conducted using only RGB masks such as the one shown as the input image in Fig. 3.2. This design provides important capabilities to our system:

- A large number of training samples can be synthetically generated by randomly removing disc units within the insulator string mask.

- A variable number of disc units can be removed from the mask, making the proposed network robust to a large number of possible defect configurations where several discs can be absent from the insulator string. This property makes this component more flexible and robust as compared to other state-of-the-art works trained directly on the original RGB images, where there is a limited amount of defect configurations.

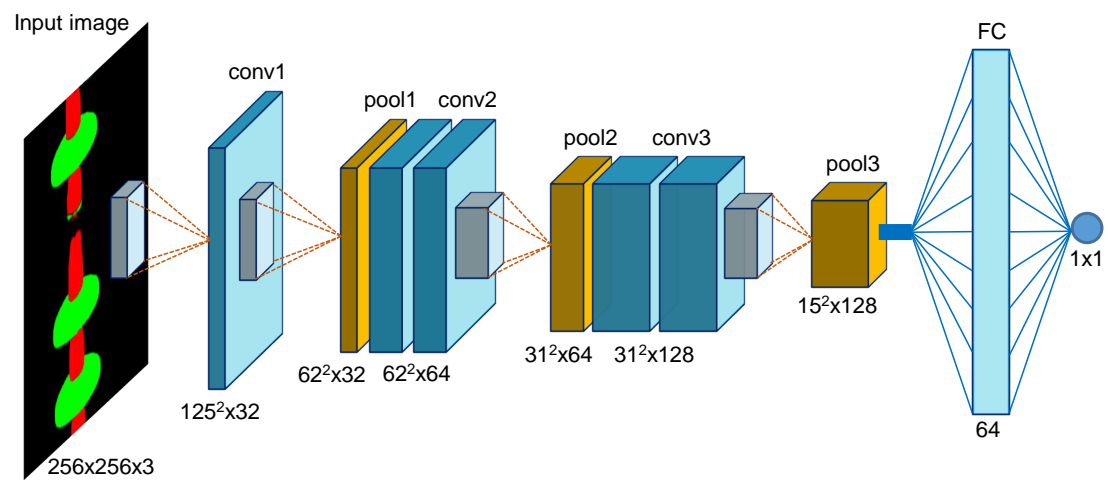

Figure 3.2: Proposed Convolutional Neural Network architecture (CNN3) for diagnosing the absence of disc units within an insulator string. The proposed CNN takes as input a segmented insulator string image and outputs the probability of discs absence. "FC" stands for fullyconnected.

The architecture of the proposed CNN for disc absence diagnosis (CNN3) is shown in Fig. 3.2. As shown in Fig. 3.2, the proposed CNN consists of 5 convolutional layers, 3 max-pooling layers, 1 fully-connected layer, and a single-unit output layer. The first layer in the architecture applies a $7 \times 7$ unpadded convolution with a stride of 2 and ReLU activation function, followed by a $3 \times 3$ max-pooling layer with a stride of 2 . The following layers alternate $7 \times 7$ convolutional layers (padded convolutions) with a stride of 1 and ReLU activation function, with $2 \times 2$ max-pooling layers with a stride of 2 . In each level of the architecture, we increase the number of feature channels by a factor of 2 until reaching 128 channels in the last convolutional and pooling layers. The resulting $15 \times 15 \times 128$ volume is flattened and introduced to the fully-connected part of the CNN, which contains 64 hidden units, featuring ReLU non-linearity as the activation function, connected to a single-unit output layer. The final output unit consists of a sigmoid unit 
which allows for providing predictions in the range $[0,1]$, where 1 indicates a fault in the insulator string, and 0 reveals that the insulator string is not defective.

Several CNN architectures have been studied, conducting a detailed analysis regarding the number and size of the convolutional layers and the size of their convolution kernels, with the intuition that larger receptive fields can be beneficial for detecting the absence of disc units within the insulator string. For this purpose, three main CNN architectures with varying kernel sizes have been evaluated, whose architecture is depicted in Table 3.1. As shown in Table 3.1. CNN1 is composed of 3 convolutional and 3 pooling layers. CNN2 architecture consists of a 10-layer CNN, in which we add one more convolutional layer in the second and third convolutional blocks (conv2 and conv3). Another representative characteristic of CNN2 is that we increase the stride of the first convolutional layer in the second convolutional block (conv2) with the purpose of reducing the number of parameters in the network. Finally, CNN3 has a similar architecture as compared to CNN2, where the stride of the first convolutional layer in the second convolutional block is restored to 1 , and we double the number of kernels in the third convolutional block (conv3). All the pooling layers are identical in the three $\mathrm{CNN}$ architectures and consist of a $3 \times 3$ maxpooling operation with a stride of 2 in the first pooling layer, and a $2 \times 2$ max-pooling operation with a stride of 2 in the second and third pooling layers. The fully-connected network at the end of each CNN is identical for the three architectures and consists of one hidden layer composed of 64 units followed by a single-unit output layer.

Table 3.1: CNN architectures designed for diagnosing the absence of disc insulator units. $k$ represents the kernel size, and $s$ is the stride. The number before the @ represents the number of kernels in the corresponding convolutional layer. "FC" refers to the fully-connected part of the CNN.

\begin{tabular}{cccc}
\hline Layer & CNN1 & CNN2 & CNN3 \\
\hline conv1 & $32 @ k \times k \times 3, s=2$ & $32 @ k \times k \times 3, s=2$ & $32 @ k \times k \times 3, s=2$ \\
pool1 & $3 \times 3, s=2$ & $3 \times 3, s=2$ & $3 \times 3, s=2$ \\
conv2 & $64 @ k \times k \times 32, s=1$ & $64 @ k \times k \times 32, s=2$ & $64 @ k \times k \times 32, s=1$ \\
pool2 & $2 \times 2, s=2$ & $64 @ k \times k \times 64, s=1$ & $64 @ k \times k \times 64, s=1$ \\
conv3 & $128 @ k \times k \times 64, s=1$ & $64 @ k \times k \times 64, s=1$ & $128 @ k \times k \times 64, s=1$ \\
pool3 & $2 \times 2, s=2$ & $64 @ k \times k \times 64, s=1$ & $128 @ k \times k \times 128, s=1$ \\
FC & $64 \times 1$ & $2 \times 2, s=2$ & $2 \times 2, s=2$ \\
\hline
\end{tabular}

The comparative analysis regarding the performance of the three CNN architectures presented in Table 3.1 is shown in Section 3.5.2.

\section{Diagnosis of damaged disc units}

In this section, we describe the strategy designed for diagnosing different types of defects in the disc units of the insulator string (e.g., disc polluted, rusted, burned, etc.) 
using a supervised learning framework. For convenience, we will name any type of disc unit defect as "damaged" from now on in the document. Insulator strings can appear under a wide range of conditions within the image (e.g., different color, texture, point of view, orientation, lighting conditions, etc.). Moreover, the great majority of insulators disposed along the power transmission lines are ceramic or made of glass, which usually causes the presence of reflections in the discs (see input image in Fig. 2.2). This constraint can significantly complicate the diagnostic process of the insulator string elements due to visual similarities with respect to certain types of disc surface pollution. In order to build a robust diagnosis system able to tackle the aforementioned constraints, in this work we propose a novel strategy based on an SCNN architecture (see Fig. 3.3). As shown in Fig. 3.3, the proposed SCNN consists of two twin CNNs with shared weights which are joined after the fully-connected layers by an energy function, which computes a distance metric between the hidden states of the twin CNNs.

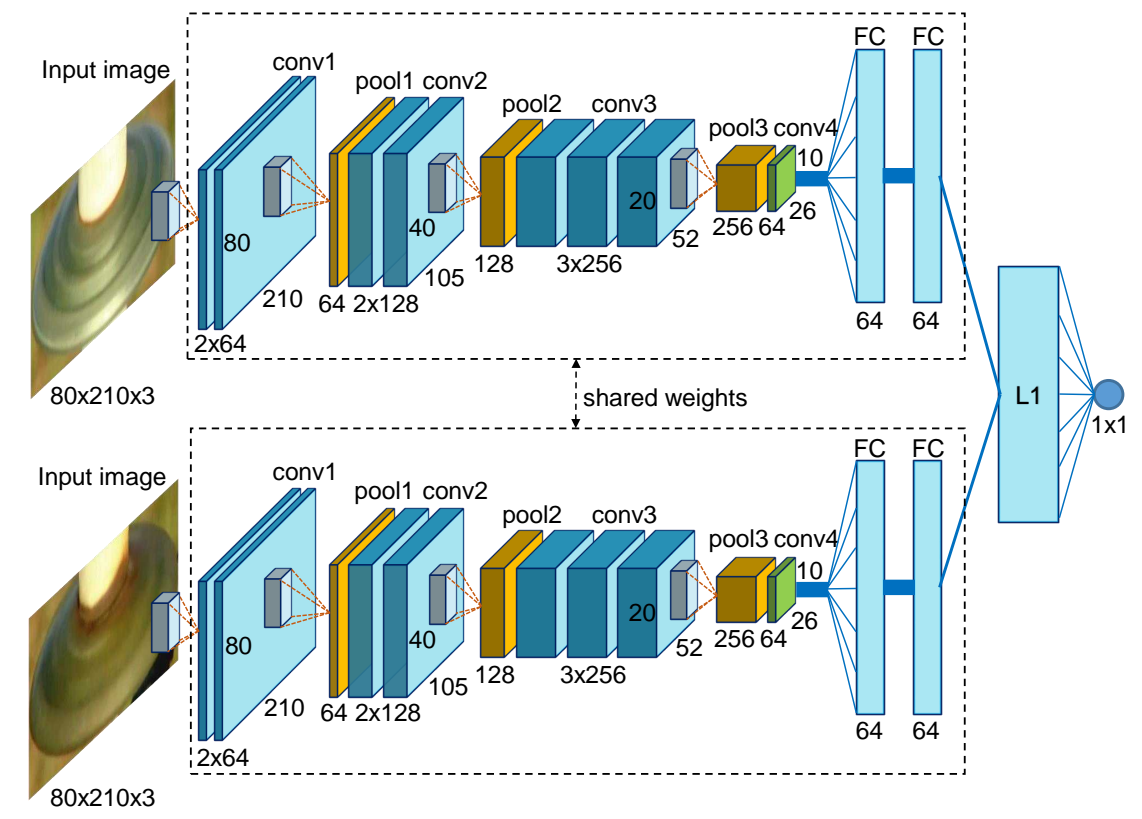

Figure 3.3: Proposed Siamese Convolutional Neural Network architecture (SCNN-c4-L1) for diagnosing damaged disc units. The SCNN takes as input a pair of disc images within the insulator string and outputs the probability of dissimilarity between the input images. "FC" stands for fully-connected.

In this work, we have evaluated different SCNN architectures and energy functions which are summarized in Table 3.2. The best performing SCNN (SCNN-c4-L1) is the one shown in Fig. 3.3, where each twin CNN follows a VGG16 architecture until the third convolutional block (conv3). After this, a max-pooling layer with kernel size and stride of 2 is followed by a $1 \times 1$ convolutional layer with Batch Normalization (Ioffe and Szegedy, 2015), which plays an important role in reducing the number of channels before the fully-connected network. The resulting volume is flattened and introduced to the fully-connected part of each twin CNN, consisting of two hidden layers of 64 units each, using ReLU activation function. Dropout with $50 \%$ probability is introduced between 
Table 3.2: CNN architectures inside each SCNN configuration, designed for diagnosing damaged disc units. $s$ represents the stride, and the number before the @ depicts the number of kernels in the corresponding convolutional layer. "FC" refers to the fully-connected part.

\begin{tabular}{cccc}
\hline Layer & SCNN-L1 $/ \chi^{2}$ & SCNN-c3-L1 $/ \chi^{2}$ & SCNN-c4-L1 $/ \chi^{2}$ \\
\hline conv1 & $32 @ 7 \times 7 \times 3, s=1$ & $64 @ 3 \times 3 \times 3, s=1$ & $64 @ 3 \times 3 \times 3, s=1$ \\
pool1 & $3 \times 3, s=2$ & $64 @ 3 \times 3 \times 64, s=1$ & $64 @ 3 \times 3 \times 64, s=1$ \\
& $64 @ 5 \times 5 \times 32, s=1$ & $128 @ 3 \times 3 \times 64, s=1$ & $128 @ 3 \times 3 \times 64, s=1$ \\
conv2 & $64 @ 5 \times 5 \times 64, s=1$ & $128 @ 3 \times 3 \times 128, s=1$ & $128 @ 3 \times 3 \times 128, s=1$ \\
pool2 & $2 \times 2, s=2$ & $2 \times 2, s=2$ & $2 \times 2, s=2$ \\
& $64 @ 5 \times 5 \times 64, s=1$ & $256 @ 3 \times 3 \times 128, s=1$ & $256 @ 3 \times 3 \times 128, s=1$ \\
conv3 & $64 @ 5 \times 5 \times 64, s=1$ & $256 @ 3 \times 3 \times 256, s=1$ & $256 @ 3 \times 3 \times 256, s=1$ \\
pool3 & $2 \times 2, s=2$ & $256 @ 3 \times 3 \times 256, s=1$ & $256 @ 3 \times 3 \times 256, s=1$ \\
conv4 & & $2 \times 2, s=2$ & $2 \times 2, s=2$ \\
FC & $64 \times 2$ & $64 \times 2$ & $64 @ 1 \times 1 \times 256, s=1$ \\
\hline
\end{tabular}

the two hidden layers. The two hidden states from the twin CNNs (denoted by $\mathbf{h}_{1}$ and $\mathbf{h}_{2}$ ) are then introduced to the L1 layer followed by a single logistic output unit. Similar to the work proposed in Taigman et al., 2014 and Koch et al., 2015, this final block of the SCNN computes a weighted L1 distance between the hidden states of the form $d\left(\mathbf{h}_{1}, \mathbf{h}_{2}\right)=\sigma\left(\sum_{i} \boldsymbol{\omega}_{i}\left|\mathbf{h}_{1}^{(i)}-\mathbf{h}_{2}^{(i)}\right|\right)$, where $\sigma$ is the sigmoid activation function, and $\boldsymbol{\omega}_{i}$ are the weights learned in the output layer. The output of the SCNN computed by the sigmoid output unit is within the range $[0,1]$, where 0 encodes the condition where the two input images are the "same", which means no defect in the discs, and 1 encodes the condition where the two input images are "different", thus, indicating the presence of a defect in one or both discs.

Within the SCNN framework, we experiment with different variants of the main architecture presented in Fig. 3.3. The main analysis is based on the evaluation of different distance layers. In this work, we evaluate the performance of the SCNNs using the L1 and the Chi-square distances. The latter, once connected to the final output layer of the SCNN, computes the similarity between hidden states using the expression: $d\left(\mathbf{h}_{1}, \mathbf{h}_{2}\right)=\sigma\left(\sum_{i} \boldsymbol{\omega}_{i}\left(\mathbf{h}_{1}^{(i)}-\mathbf{h}_{2}^{(i)}\right)^{2} /\left(\mathbf{h}_{1}^{(i)}+\mathbf{h}_{2}^{(i)}+\epsilon\right)\right)$, where $\sigma$ is the sigmoid activation function, $\boldsymbol{\omega}_{i}$ are the weights learned in the output layer, and $\epsilon=10^{-6}$ is introduced to obtain numeric stability. Within the SCNN framework, three main neural network variants have been designed, depending on the architecture of the twin CNNs within the SCNN (see Table 3.2. The first SCNN architecture, named SCNN-L1 $/ \chi^{2}$, has fewer parameters as compared to other SCNN variants and consists of 3 convolutional blocks (conv1 to conv3 in Fig. 3.3) of $1 \times 32,2 \times 64$, and $2 \times 64$ respectively. The second SCNN architecture studied, named SCNN-c3-L1 $/ \chi^{2}$, follows the VGG16 architecture until the third convolutional block (conv3), consisting of $2 \times 64,2 \times 128$, and $3 \times 256$ convolutional blocks. The final SCNN architecture analyzed, named SCNN-c4-L1 $/ \chi^{2}$, introduces a fourth $1 \times 1$ 
convolutional block as explained previously in this section. All the CNNs within the different SCNN architectures finish with a fully-connected part composed of 2 hidden layers with 64 units each. Finally, we analyze the effect of transfer learning in the SCNN-c3$\mathrm{L} 1 / \chi^{2}$ and the SCNN-c4-L1 $/ \chi^{2}$ network variants, which are evaluated with and without pre-training using the VGG16 network previously trained on ImageNet.

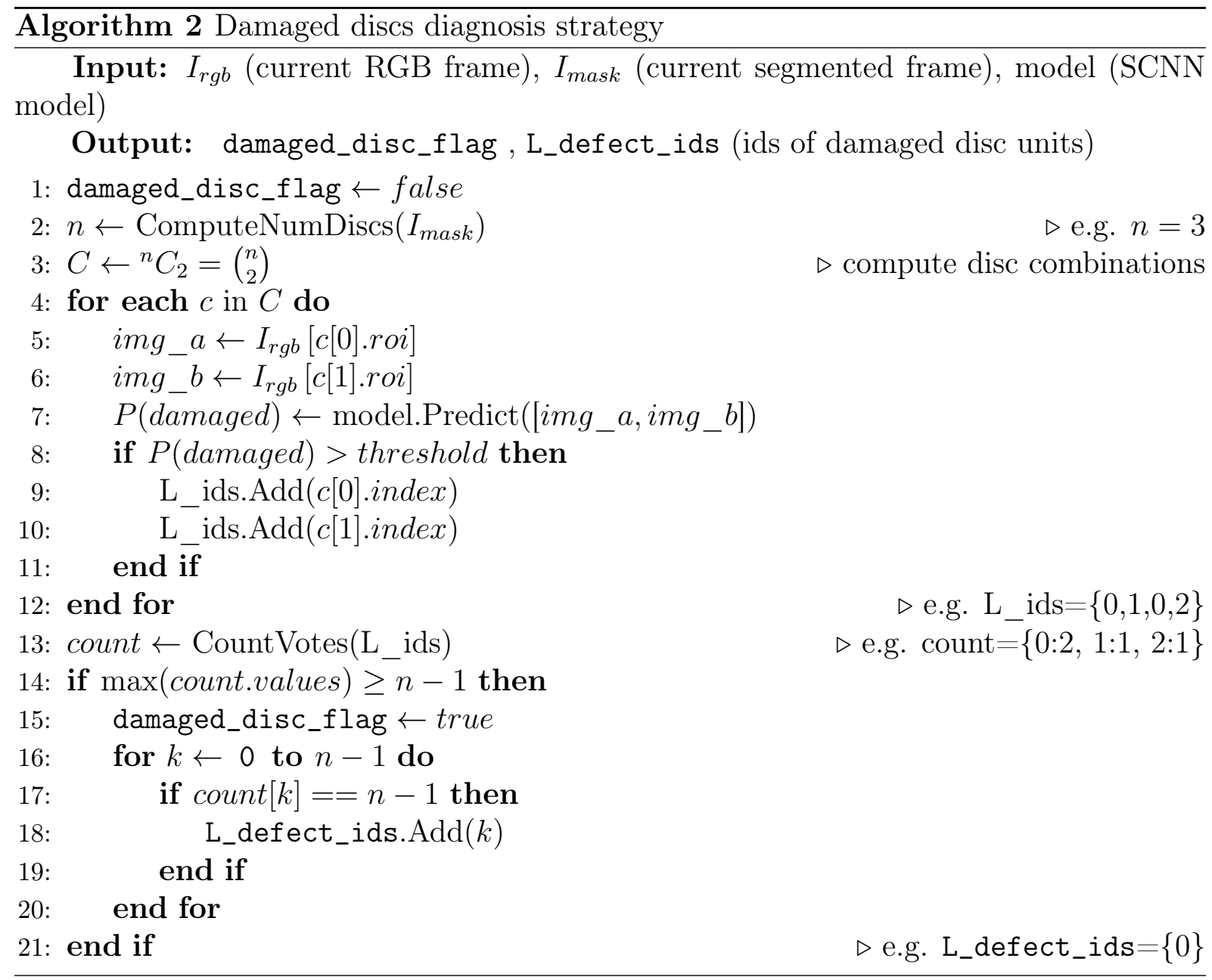

In order to identify damaged disc units and locate them within the insulator string, our strategy uses Algorithm 2, which is based on computing all the combinations between pairs of discs within the insulator string in the current frame using ${ }^{n} C_{2}=n ! /(2 !(n-2) !)$, where $n$ is the number of detected discs in the current frame. Each of these combinations of cropped images is introduced to the SCNN model for classifying them as "damaged" or not. From these combinations, we extract a list of the corresponding disc pairs that have been classified as "damaged" by the proposed SCNN and compute the most frequent elements in the list. If the number of occurrences of these elements is greater or equal to $n-1$, it means that all remaining discs within the insulator string have voted for the specific disc as being "damaged", and thus the defective disc is located. It should be noted that the proposed strategy needs at least two disc units in order to start diagnosing the insulator string, and at least three disc units in order to precisely locate the defective 
disc in the image. In the former case, when only two disc units are visible at the current frame, we mark the corresponding pair of discs as "damaged", in case there is a defect.

It should be noted that despite the proposed strategy can increase the computational cost of the whole system since all the disc pairs combinations are introduced to the SCNN, it provides an additional filtering stage which allows for the removal of possible false positives.

\subsubsection{Semi-Supervised Anomaly Detection}

In this section, we describe in detail the proposed approach for diagnosing damaged disc units obtained by using a semi-supervised learning framework. The proposed framework is shown in Fig. 3.4. and consists of a two-stage training process. In the first stage, a Convolutional Autoencoder (CAE) is trained for modeling the probability distribution of non-damaged disc images. Subsequently, the learned encoder network from the CAE is integrated into a siamese architecture for extracting the encodings used in the second training stage. The latter stage is used for training a One-Class SVM using only non-damaged disc images.

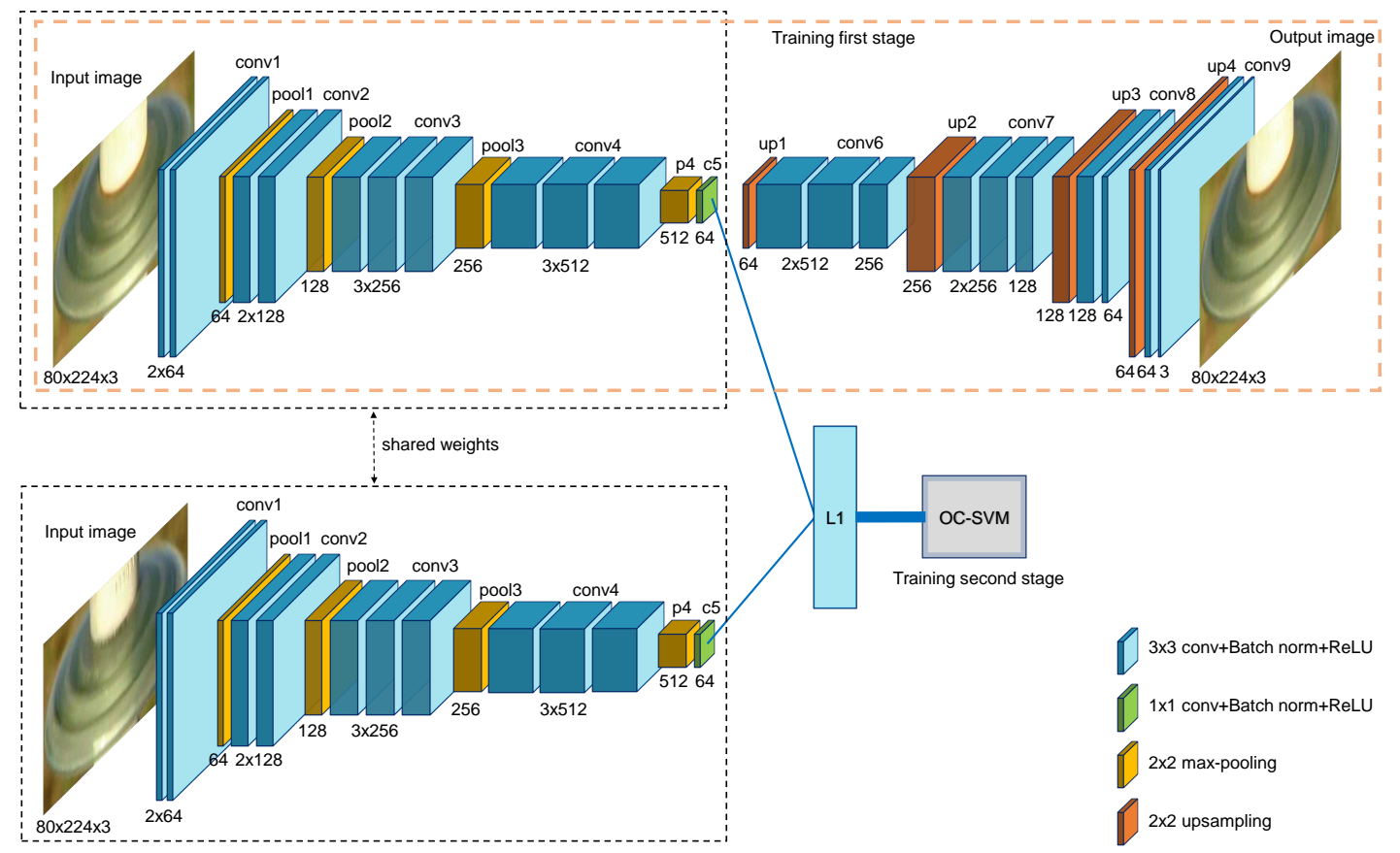

Figure 3.4: Proposed Siamese Convolutional Autoencoder architecture (SCAE-c5L1_OC_SVM) for diagnosing damaged disc units. The SCAE has been obtained by conducting a two-stage training. First, a convolutional autoencoder is trained on healthy disc examples. Second, the weights of the encoder network of the CAE are transferred to a Siamese architecture which is connected to an L1 layer followed by an OC-SVM. Training the OC-SVM on non-damaged disc examples constitutes the second training stage.

Regarding the CAE proposed in the first training stage, we have analyzed two different architectures. The architecture shown in Fig. 3.4 is the one which provides the best 
diagnosis results using the semi-supervised learning framework as can be reviewed in Section 3.5.3. This architecture consists of a reduced version of a SegNet encoder-decoder network, in which the encoder path follows the VGG16 architecture until the fourth convolutional block (conv4), that is, alternating convolutional layers of $3 \times 3$ convolution kernels with a stride of 1 followed by $2 \times 2$ max-pooling layers with a stride of 2 . In all the convolutional layers, we used Batch Normalization and ReLU activation function. As in the VGG16 architecture, the number of channels is increased by a factor of 2 until reaching 512 channels in the fourth convolutional block. The final block in the encoder network consists of a $1 \times 1$ convolutional layer (see $c 5$ in Fig. 3.4), and is designed for reducing the dimensionality of the final encodings used for training the OC-SVM. The decoder network has a similar architecture as the encoder one, alternating $3 \times 3$ convolutional layers with $2 \times 2$ upsampling layers using nearest-neighbor interpolation. The proposed CAE network is trained as described in Section 3.5 .3 for minimizing the Mean Squared Error (MSE) between the output predicted by the network and the input image.

Once the CAE has been trained, we extract the encoder network from the CAE and duplicate it to build a Siamese architecture as shown in Fig. 3.4. As in the case of the SCNN presented in Section 3.4.1, we connect the twin encoder networks to an energy function (L1 in this case) which is used to compute the final encodings for training the OCSVM. The objective of the proposed siamese architecture trained in the semi-supervised fashion is the same as stated in Section 3.4.1 for the SCNN, and is to provide robustness to the variability of the inputs to the network.

The model obtained following the two-stage training described above is referred to as SCAE-L1_OC_SVM since it involves a first stage for modeling the probability distribution of the inputs using a CAE and a second stage using a Siamese architecture integrating an L1 layer to compute the final encodings used by the OC-SVM in the semisupervised learning framework. Using the previously described learning framework, two variants of the proposed SCAE-L1_OC_SVM have been conceived. As shown in Table 3.3, we first design a lightweight SCAE (SCAE-c4-L1_OC_SVM), which follows the VGG16 architecture until the third convolutional block. After this, a fourth convolutional layer is applied using 16 kernels of $1 \times 1 \times 256$, which allows obtaining encodings of length $10 \times 28 \times 16=4480$. The second variant (SCAE-c5-L1_OC_SVM) has been previously described and allows obtaining encodings of the same length $5 \times 14 \times 64=4480$.

\subsection{Experiments and Results}

In this section, we present the experiments conducted and the results obtained in the evaluation of the fault diagnosis methods presented in this chapter. First, the experimental setup is described in Section 3.5.1. Subsequently, the experiments and results obtained in the the evaluation of the absent disc diagnosis component and the damaged disc diagnosis component are illustrated in Section 3.5.2 and Section 3.5.3, respectively. Finally, Section 3.5.4 describes the diagnosis results obtained by the complete system. A video of the reported results is available in: https://vimeo.com/album/5782445. 
Table 3.3: CNN architectures designed for each SCAE configuration, used for diagnosing damaged disc units. $s$ represents the stride, and the number before the @ depicts the number of kernels in the corresponding convolutional layer.

\begin{tabular}{ccc}
\hline Layer & SCAE-c4-L1_OC_SVM & SCAE-c5-L1_OC_SVM \\
\hline \multirow{2}{*}{ conv1 } & $64 @ 3 \times 3 \times 3, s=1$ & $64 @ 3 \times 3 \times 3, s=1$ \\
pool1 & $64 @ 3 \times 3 \times 64, s=1$ & $64 @ 3 \times 3 \times 64, s=1$ \\
& $2 \times 2, s=2$ & $2 \times 2, s=2$ \\
conv2 & $128 @ 3 \times 3 \times 64, s=1$ & $128 @ 3 \times 3 \times 64, s=1$ \\
pool2 & $28 \times 3 \times 3 \times 128, s=1$ & $128 @ 3 \times 3 \times 128, s=1$ \\
& $256 @ 3 \times 3 \times 128, s=1$ & $256 @ 3 \times 3 \times 128, s=1$ \\
conv3 & $256 @ 3 \times 3 \times 256, s=1$ & $256 @ 3 \times 3 \times 256, s=1$ \\
& $256 @ 3 \times 3 \times 256, s=1$ & $256 @ 3 \times 3 \times 256, s=1$ \\
pool3 & $2 \times 2, s=2$ & $2 \times 2, s=2$ \\
& & $512 @ 3 \times 3 \times 256, s=1$ \\
conv4 & $16 @ 1 \times 1 \times 256, s=1$ & $512 @ 3 \times 3 \times 512, s=1$ \\
& & $512 @ 3 \times 3 \times 512, s=1$ \\
pool4 & & $2 \times 2, s=2$ \\
conv5 & & $64 @ 1 \times 1 \times 512, s=1$ \\
\hline
\end{tabular}

\subsubsection{Experimental Setup}

All the networks described in Section 3.4 have been implemented in Python and trained using the Keras deep learning framework. The one-class SVM has been trained using the LIBSVM library (Chang and Lin, 2011). All the experiments have been conducted on an Nvidia GeForce GTX 1080Ti GPU under Ubuntu 16.04 LTS, using OpenCV 4.0.0 for image processing tasks.

\subsubsection{Diagnosis of absent disc units}

In this work, we address the problem of diagnosing the absence of disc insulator units within an insulator string as a classification problem, where the objective is to identify the occurrence of this fault at frame-level. For this purpose, the network in charge of diagnosing this defect takes as input the output mask generated by the insulator string segmentation component and outputs the probability of fault occurrence.

\section{Dataset}

Owing to the relatively small amount of defective images in our dataset, we have left the real images containing defective samples only for evaluation purposes and created a synthetic dataset for training and validating the component responsible for diagnosing the absence of disc insulator units. This dataset is composed of 1200 negative images (non-defective) and 1200 positive images (i.e., with one or more absent disc units) of 
$256 \times 256$ pixels (see Fig. 3.5 for an example). Inside this dataset, the negative samples have been obtained by doing inference over some of the semantic segmentation networks presented in Table 2.3 for the RGB training images used for semantic segmentation. We use several networks at different epochs of the training process for obtaining a heterogeneous dataset of negative samples. In addition, $10 \%$ of these images have been manually altered by removing some pixels of the disc and cap classes in order to simulate errors in the semantic segmentation stage (see the last image in Fig. 3.5a). Using the negative samples, we generate the dataset of positive samples, where $60 \%$ of the 1200 images have been automatically generated using computer vision techniques by alternatively removing some of the disc units within an insulator string. In addition, random noise is added to some of these images. The remaining $40 \%$ of the positive samples have been manually generated by removing the pixels belonging to some of the caps and disc units within the insulator string.
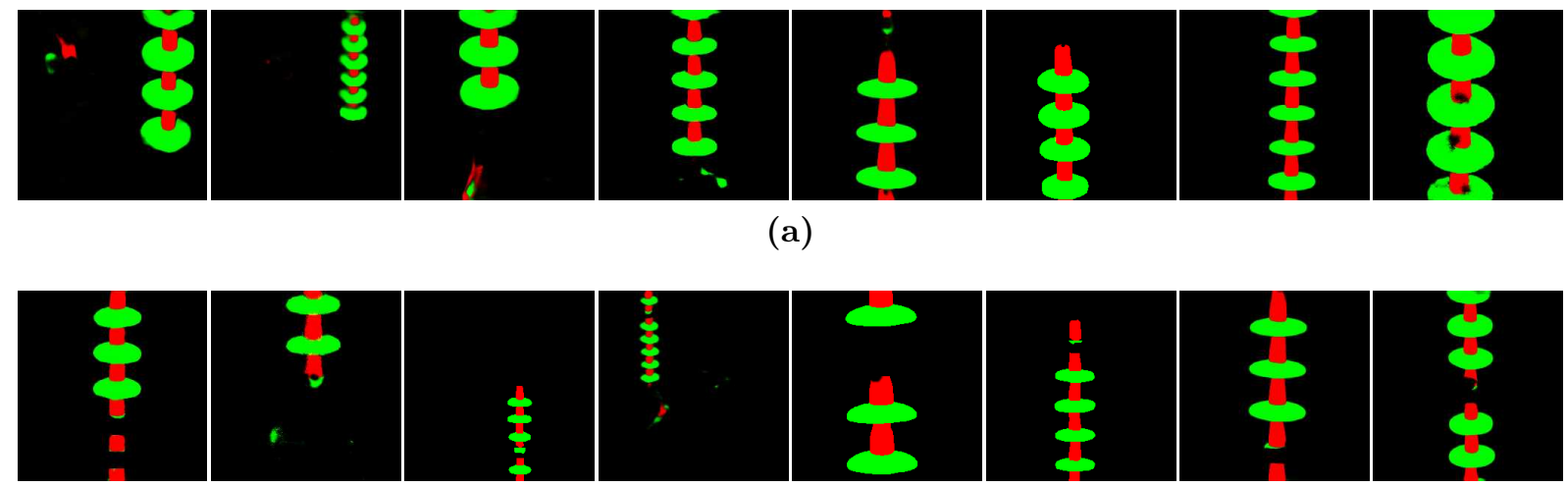

(b)

Figure 3.5: Examples of images used for training the component responsible for diagnosing the absence of disc insulator units. Images containing false positives and false negatives are included in the training set to provide robustness to possible errors that might appear in the insulator segmentation stage. (a) Negative samples (non-defective images). (b) Positive samples (defective images) synthetically generated to reproduce different defect configurations relative to the absence of disc units.

On the other hand, the evaluation of the different CNNs designed for diagnosing the absence of disc insulator units is conducted on a test set composed of 400 images, where the samples are equally distributed between the negative and positive classes (i.e., 200 images for each class).

\section{Training methodology}

In this work, we consider the problem of diagnosing the absence of disc insulator units as a binary classification problem, in which the current frame analyzed can be defective, which means that one or several disc units are absent from the insulator string, or correct, which implies that the insulator string is in good condition. As a binary classification problem, we use a binary cross-entropy loss function (see Eq. 3.1), which is minimized during a training process of 60 epochs using the Adam optimizer (see Section A.1.3 of 
Appendix A for a detailed explanation) with a base learning rate of $2^{-4}$, and the rest of hyperparameters as described in the original work (Kingma and Ba, 2014). The minibatch size $(\mathrm{M})$ utilized in each update of the optimization process is 128 images.

$$
\mathcal{L}(\mathbf{x} ; \mathbf{w})=-\frac{1}{M} \sum_{i=1}^{M}\left[y\left(\mathbf{x}^{(i)}\right) \log \hat{y}\left(\mathbf{x}^{(i)} ; \mathbf{w}\right)+\left(1-y\left(\mathbf{x}^{(i)}\right)\right) \log \left(1-\hat{y}\left(\mathbf{x}^{(i)} ; \mathbf{w}\right)\right)\right]
$$

where $y \in\{0,1\}$ is the ground truth label, being 0 whenever the input mask is not defective or 1 otherwise, $\hat{y}$ is the output predicted by the CNN given the input $\mathbf{x}^{(i)}$, and w represents the trainable parameters of the network.

In each experiment, we perform a randomly stratified split of the training dataset (i.e., 2400 negative and positive samples) into training and validation sets, where the percentage of samples in each set is $80 \%$ and $20 \%$, respectively.

\section{Evaluation metrics}

For the sake of obtaining an appropriate comparison of the CNN architectures considered, we use standard metrics widely applied in the research community for evaluating binary classifiers. These metrics are summarized here for a better understanding of the results:

- Accuracy: $(t p+t n) /(t p+t n+f p+f n)$

- Precision: $t p /(t p+f p)$

- Recall: $t p /(t p+f n)$

- $F_{1}$ score: $2 t p /(2 t p+f p+f n)$

- Area under the Receiver Operating Characteristic (ROC) curve (ROC-AUC): this curve depicts the True Positive Rate (TPR) versus the False Positive Rate (FPR), showing how the correctly classified positive examples (TPR) vary with respect to the incorrectly classified negative examples (FPR).

where $t p, t n, f p$, and $f n$ stand for true positive, true negative, false positive, and false negative, respectively.

All the metrics utilized for the evaluation of the different models have been computed using scikit-learn (Pedregosa et al., 2011).

\section{Results in the diagnosis of absent discs}

The results obtained after the evaluation of the different CNN architectures considered are depicted in Table 3.4, which shows the mean and standard deviation after conducting 5 trainings for each of the $\mathrm{CNN}$ configurations. According to these results, the network CNN3 with a kernel size of $7 \times 7$ outperforms the other CNN configurations, having an average ROC-AUC of $99.89 \%$. The results presented in Table 3.4 also reveal the 
considerable effect of the number of convolutional layers and the size of the convolution kernels in the performance of the different CNN architectures. The former is evidenced when moving from CNN1 to CNN3, in which the $F_{1}$ score is improved by $4.4 \%, 1.9 \%$, and $2.5 \%$ using kernel sizes of $3 \times 3,5 \times 5$, and $7 \times 7$, respectively. The effect of the size of the convolution kernels is noted when increasing the size from $3 \times 3$ to $7 \times 7$ which leads to an increment in the $F_{1}$ score of $5.7 \%, 3.2 \%$, and $3.8 \%$ for the CNN1, CNN2, and CNN3 architectures respectively. This effect is also evidenced when analyzing the ROC-AUC, which experiences an increment of $1.9 \%, 0.6 \%$, and $1.2 \%$ for the CNN1, CNN2, and CNN3 respectively. These results are consistent with the intuition that larger receptive fields can benefit the diagnosis of this type of defect.

Table 3.4: Mean and standard deviation obtained by averaging the results of 5 different models (5 trainings) for each CNN configuration while diagnosing the absence of disc insulator units on a test set of 400 images. The number of parameters of the network is expressed in millions. Best results are indicated in bold.

\begin{tabular}{cccccccc}
\hline CNN arch. & kernel size & Accuracy (\%) & Precision (\%) & Recall (\%) & $F_{1}$ score $(\%)$ & ROC-AUC $(\%)$ & \#param (M) \\
\hline \multirow{3}{*}{ CNN1 } & $3 \times 3$ & $90.75 \pm 0.91$ & $91.29 \pm 1.03$ & $90.13 \pm 2.56$ & $90.68 \pm 1.03$ & $97.07 \pm 0.36$ & 1.94 \\
& $5 \times 5$ & $94.63 \pm 0.32$ & $93.76 \pm 0.56$ & $95.75 \pm 0.65$ & $94.74 \pm 0.24$ & $98.59 \pm 0.19$ & 2.10 \\
& $7 \times 7$ & $96.38 \pm 0.14$ & $96.05 \pm 1.01$ & $96.75 \pm 0.96$ & $96.39 \pm 0.13$ & $99.01 \pm 0.26$ & 2.35 \\
\hline \multirow{3}{*}{ CNN2 } & $3 \times 3$ & $94.44 \pm 0.85$ & $92.09 \pm 1.22$ & $97.38 \pm 0.75$ & $94.66 \pm 0.85$ & $98.72 \pm 0.20$ & 0.39 \\
& $5 \times 5$ & $96.72 \pm 0.70$ & $95.58 \pm 1.45$ & $98.06 \pm 0.78$ & $96.80 \pm 0.71$ & $99.40 \pm 0.26$ & 0.56 \\
& $7 \times 7$ & $97.85 \pm 0.45$ & $97.44 \pm 1.02$ & $98.30 \pm 0.84$ & $97.86 \pm 0.44$ & $99.36 \pm 0.07$ & 0.91 \\
\hline \multirow{2}{*}{ CNN3 } & $3 \times 3$ & $95.06 \pm 0.38$ & $95.12 \pm 0.26$ & $95.00 \pm 0.58$ & $95.06 \pm 0.38$ & $98.65 \pm 0.05$ & 2.12 \\
& $5 \times 5$ & $96.84 \pm 0.35$ & $96.54 \pm 1.01$ & $97.19 \pm 0.59$ & $96.86 \pm 0.33$ & $99.15 \pm 0.25$ & 2.61 \\
\hline
\end{tabular}

In order to provide better insight into each CNN architecture studied in this section, in Fig. 3.6 we show the ROC curves generated by the best models selected from the 5 training experiments conducted on each CNN configuration. The results presented in Fig. 3.6 provide additional information about the capabilities of each CNN architecture, showing the high AUC obtained for the CNN2 and CNN3 architectures with $5 \times 5$ and $7 \times 7$ kernel sizes. Taking into account the results presented in Table 3.4 and Fig. 3.6, CNN3_7x7 is selected as the network utilized in the final system for diagnosing the absence of disc insulator units.

Some prediction results generated by the CNN3_7x7 model on a subset of representative images from the test set are shown in Fig. 3.7. In this figure, we also show the real RGB images (see Fig. 3.7a) from which the masks are computed using the Up-Net_vgg16 model (see Fig. 3.7b). Furthermore, Fig. 3.7b provides a complementary validation of the performance obtained by the insulator string segmentation component (Up-Net_vgg16).

\subsubsection{Diagnosis of damaged disc units}

In this section, we describe the experiments that have been conducted in order to select the most appropriate network for the task of diagnosing the presence of damaged discs within the insulator string. As explained in Section 3.4.1, we address the problem of diagnosing this type of defect by designing a siamese architecture, which receives as 


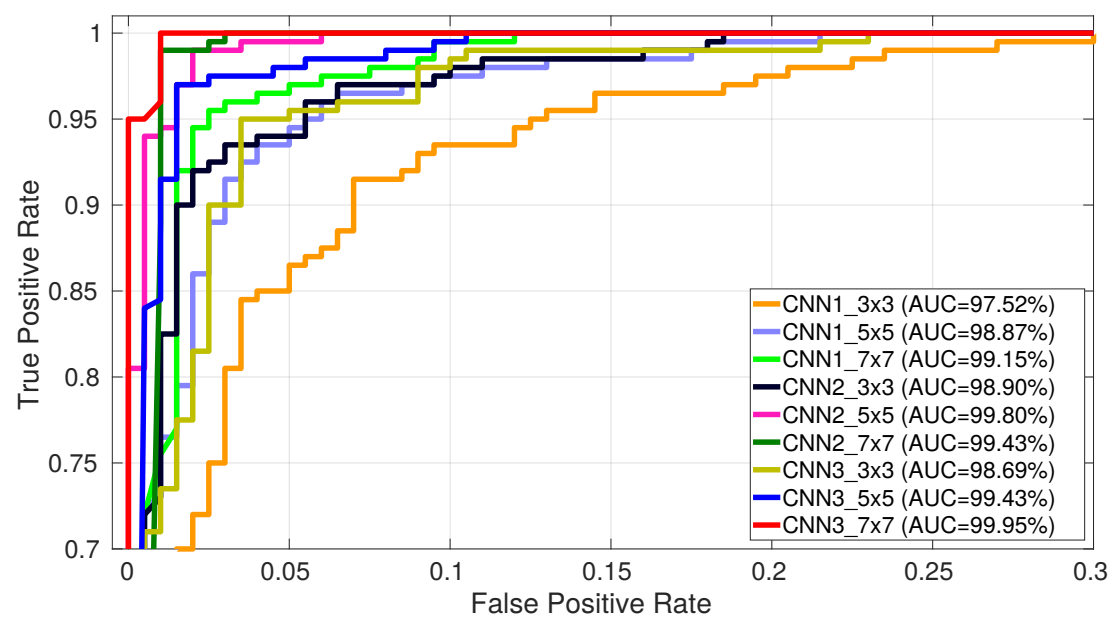

Figure 3.6: ROC curves generated by the best model for each CNN configuration tested for the disc absence defect diagnosis (best seen in color).
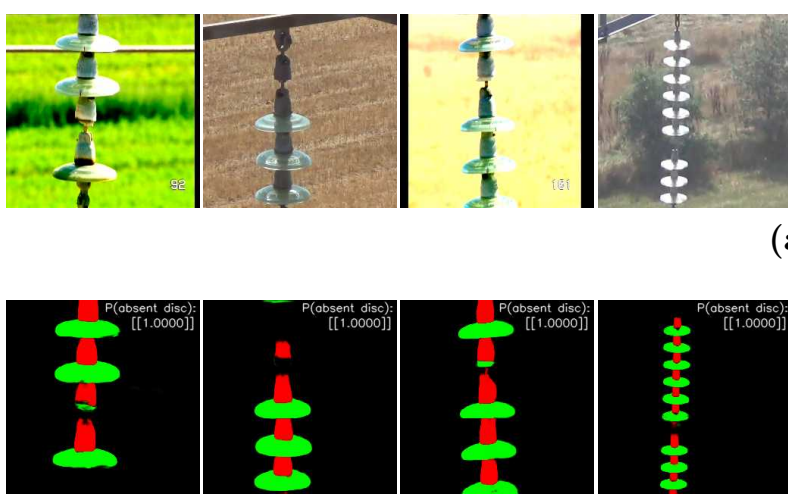

(b)

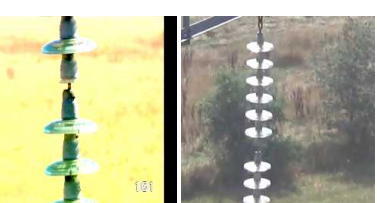

(a)

b)
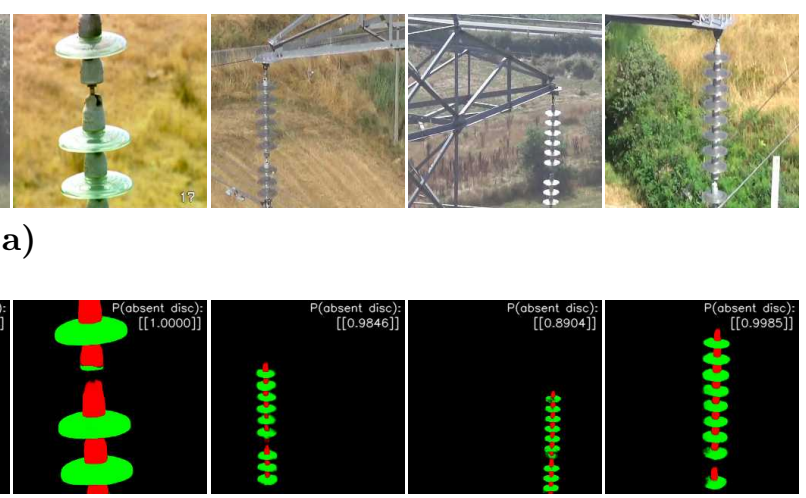

Figure 3.7: Examples of images from the test set used for evaluating the component responsible for diagnosing the absence of disc units. (a) Original RGB images from which the masks for evaluation purposes are extracted. (b) Masks generated by the insulator string segmentation component (Up-Net_vgg16) and probabilities predicted by the selected model for diagnosing the absence of disc units (CNN3_7x7). As shown in the two right-most images in Fig. 3.7b, the proposed CNN3_7x7 is able to correctly handle false positives and false negatives that might appear in the insulator segmentation stage.

input a pair of disc images and outputs a probability which encodes the dissimilarity between the input images, where 0 represents that the two input images are the "same" (non-defective) and 1 when they are "different" (defective).

\section{Dataset}

In order to build the dataset for diagnosing damaged discs, we leverage from the annotations performed for training the insulator string segmentation networks (see Section 2.5.3). Thus, from the 160 images annotated for semantic segmentation purposes, we extract all the discs within the insulator strings of these images, yielding 579 and 
18 non-damaged and damaged discs, respectively. After that, we generate all possible combinations of disc pairs within an insulator string for the corresponding image. This process allows obtaining a total of 802 images of negative (non-damaged) pairs and 95 positive (damaged) pairs (see Fig. 3.8a and 3.8b).
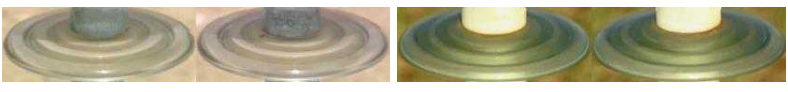

(a)
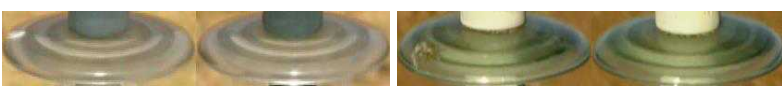

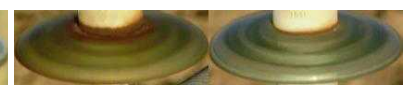

(b)

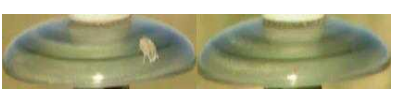

(b)
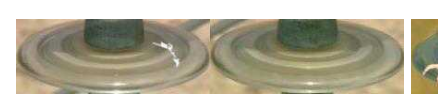
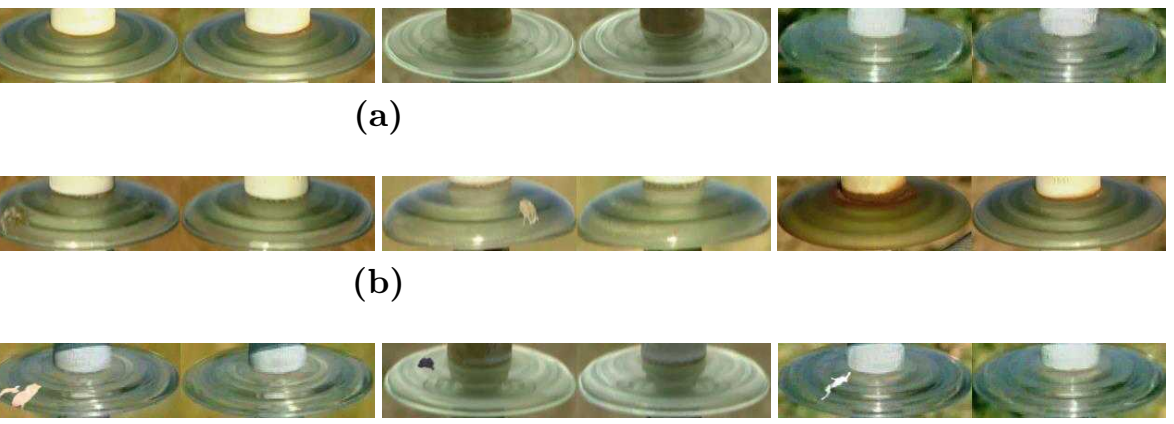

(c)

Figure 3.8: Examples of images used for training the proposed SCNN model. (a) Real examples of non-damaged pairs. (b) Real examples of damaged pairs. (c) Examples of damaged pairs which contain synthetic defects. Images of disc units showing a small degree of pollution due to bird excrements and with rust on their surface are used for training the proposed SCNN.

In order to deal with this highly imbalanced dataset, several strategies have been applied and evaluated:

- Synthetic samples generation: we manually generate synthetic defects on the disc surface by mimicking the appearance of real defective discs. For this purpose, 125 synthetic damaged disc images were created by placing synthetic defects on top of healthy insulator discs. After this, the 125 images generated were combined with non-defective disc images obtaining a total of 762 images of defective pairs (see Fig. $3.8 \mathrm{c}$ ), which are merged with the 95 real defective pairs in order to obtain a total of 857 defective pairs for training purposes.

- Training using only real or only synthetic data: the second strategy studied in this section consists of training the SCNNs taking into account only real or only synthetic data. In the first case, the training is performed using the dataset composed of 802 non-damaged pairs and 95 damaged pairs. This strategy implies the modification of the loss function used for training the SCNNs for classification (i.e., binary crossentropy loss) in order to tackle the problems derived from a highly imbalanced dataset. This new loss function is explained in Section 3.5.3. In the second case, the training is performed using the dataset composed of 802 non-damaged pairs and 762 damaged pairs.

On the other hand, in order to evaluate all the networks considered for diagnosing damaged discs, a test set of 193 images provided by an electric company has been used, which is composed of 133 non-damaged and 60 damaged discs. These images are combined in pairs as in the training set, yielding 245 image pairs. As in the case of the training set, this test set is also imbalanced, being composed of 162 images of non-damaged disc pairs and 83 damaged pairs, containing only real defective and non-defective images. 


\section{Baseline methods}

Additionally, in order to compare the performance of the proposed SCNN framework to traditional classification methods such as CNNs, we evaluate the best performing SCNN model (SCNN-c4-L1_vgg16) against its analogous CNN (CNN-c4_vgg16). The latter is built by extracting one of the twin CNNs from the SCNN-c4-L1_vgg16 and connecting it directly to the output unit (i.e., omitting the L1 layer), yielding the CNN-c4_vgg16.

\section{Training methodology}

Regarding the supervised learning framework, the SCNN training procedure is almost identical to the one described previously in Section 3.5.2 for the CNNs used for diagnosing the absence of disc insulator units. We also model the diagnosis of damaged discs as a binary classification problem, where the pair of images introduced to the siamese network $\left(\mathbf{x}_{1}\right.$ and $\left.\mathbf{x}_{2}\right)$ can be classified as damaged or non-damaged. Thus, we use a binary cross-entropy loss function, which is modified according to Eq. 3.2 for training using an imbalanced dataset. In all cases, we minimize the corresponding loss using the Adam optimizer during a training process of 60 epochs, using a minibatch size (M) of 128 images, and considering a base learning rate of $10^{-4}$.

$$
\begin{array}{r}
\mathcal{L}\left(\mathbf{x}_{1}, \mathbf{x}_{2} ; \mathbf{w}\right)=-\frac{1}{M} \sum_{i=1}^{M}\left[y\left(\mathbf{x}_{1}^{(i)}, \mathbf{x}_{2}^{(i)}\right) \log \hat{y}\left(\mathbf{x}_{1}^{(i)}, \mathbf{x}_{2}^{(i)} ; \mathbf{w}\right)\right. \\
\left.+\alpha\left(1-y\left(\mathbf{x}_{1}^{(i)}, \mathbf{x}_{2}^{(i)}\right)\right) \log \left(1-\hat{y}\left(\mathbf{x}_{1}^{(i)}, \mathbf{x}_{2}^{(i)} ; \mathbf{w}\right)\right)\right]
\end{array}
$$

where $y \in\{0,1\}$ represents the ground truth label, which is 0 whenever $\mathbf{x}_{1}$ and $\mathbf{x}_{2}$ are the "same" (non-damaged) or 1 otherwise, $\hat{y}$ is the output prediction of the SCNN given the inputs $\mathbf{x}_{1}^{(i)}$ and $\mathbf{x}_{2}^{(i)}, \mathbf{w}$ represents the trainable parameters of the network, and $\alpha=1$ for all the network variants except when training the SCNN-c4-L1_vgg16 model using only real damaged disc defects, where $\alpha=0.002$ has been empirically found.

The CNN-c4_vgg16 used in the comparative analysis has been trained using the 579 and 143 (18 real, plus 125 synthetic) images of non-damaged and damaged discs, respectively. The set of damaged discs is augmented by a horizontal flip procedure, doubling the number of defective images. Regarding the training methodology, the CNN-c4_vgg16 is trained using a binary cross-entropy loss, which is minimized using the same optimization method with the same hyperparameters as in the training process of the SCNN.

With respect to the semi-supervised learning framework, the two-stage training is conducted as follows. First, the CAE is trained using the 579 non-damaged disc images where the objective of the training process is modeled as a regression problem. Thus, we use an MSE loss function (see Eq. 3.3), where the objective is to minimize the pixel-wise difference between the output generated by the CAE and the input to the network.

$$
\mathcal{L}(\mathbf{x} ; \mathbf{w})=\frac{1}{M} \sum_{i=1}^{M}\left(\hat{\mathbf{y}}\left(\mathbf{x}^{(i)} ; \mathbf{w}\right)-\mathbf{x}^{(i)}\right)^{2}
$$

where $\mathbf{x}^{(i)}$ depicts the input image to the CAE and $\hat{\mathbf{y}}$ is the output given by the CAE. 
Once the CAE is trained, we build the SCAE as described in Section 3.4.2 and use the learned weights of the encoder network to extract the encodings that are utilized for training the OC-SVM. The training procedure of the OC-SVM for detecting anomalies consists of finding a hyperplane in the feature space that best separates the normal examples from the origin (Schölkopf et al., 2001; Manevitz and Yousef, 2001). We summarize here the main quadratic programming objective function used for training the OC-SVM, and refer the reader to the previous references for a deeper understanding.

$$
\begin{array}{ll}
\min _{\boldsymbol{\omega}, \boldsymbol{\xi}, \rho} & \frac{1}{2} \boldsymbol{\omega}^{T} \boldsymbol{\omega}-\rho+\frac{1}{\nu l} \sum_{i=1}^{l} \xi_{i} \\
\text { s.t. } & \boldsymbol{\omega}^{T} \Phi\left(\mathbf{x}_{i}\right) \geq \rho-\xi_{i}, \quad \xi_{i} \geq 0
\end{array}
$$

where $\nu$ is a regularization parameter that governs the fraction of training examples that can be classified as anomalies, $\xi_{i}$ are the slack variables, and $\Phi(\cdot)$ is a non-linear mapping function which allows to map the input data to a higher dimensional space where the data can be separable by the hyperplane defined by $\boldsymbol{\omega}$ and $\rho$. In the dual formulation of the SVM one can define a kernel of the form $K\left(x_{i}, x_{j}\right)=\phi\left(x_{i}\right)^{T} \phi\left(x_{j}\right)$, where $\left(x_{i}, x_{j}\right)$ are points in the input feature space. In this work, we have selected the Radial Basis Function (RBF) kernel as the non-linear mapping function. The RBF kernel is computed using the following expression: $K\left(x_{i}, x_{j}\right)=\exp \left(-\gamma\left\|x_{i}-x_{j}\right\|^{2}\right)$, where the parameter $\gamma$ models the width of the Gaussian kernel.

Taking into account the formulation presented in Eq. 3.4, the training process of the OC-SVM has been performed by conducting a grid search procedure over the parameters $\nu$ and $\gamma$. The results obtained in this training process are shown in Fig. 3.9 .

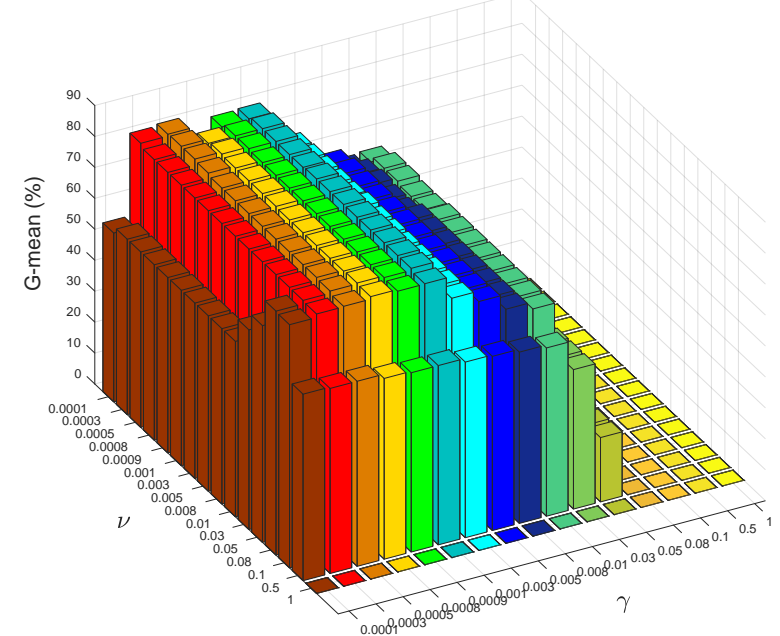

(a)

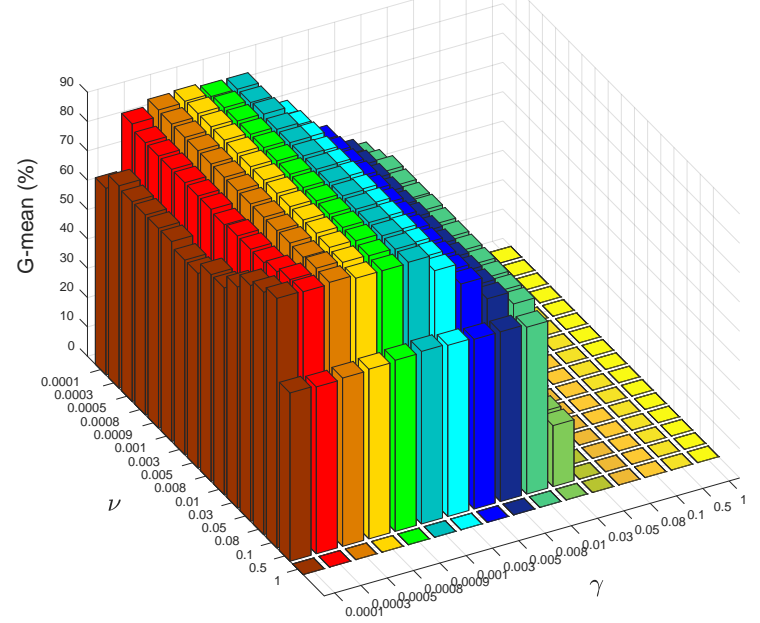

(b)

Figure 3.9: Parameter selection for the One-Class SVM model. (a) Parameter selection for the SCAE-c4-L1_OC_SVM, obtaining $\nu_{o p t}=3^{-4}$ and $\gamma_{o p t}=5^{-4}$. (b) Parameter selection for the SCAE-c5-L1_OC_SVM, obtaining $\nu_{o p t}=3^{-3}$ and $\gamma_{o p t}=8^{-4}$. 
Using the optimal values of the parameters $\nu$ and $\gamma$ for each of the considered SCAE architectures, a final training experiment is conducted on the image set used for training the SCNN model, obtaining a G-mean score of $82.09 \%$ for the SCAE-c4-L1_OC_SVM and $86.00 \%$ for the SCAE-c5-L1_OC_SVM. Further results for the final test set can be reviewed in Table 3.6.

\section{Evaluation metrics}

As we deal with a slightly imbalanced dataset for evaluation purposes (test set), in which the number of negative samples doubles the number of the positive ones, in this section we consider several metrics, computed using scikit-learn, that are usually utilized in the state of the art for imbalanced datasets (Nguyen et al., 2009):

- True Negative Rate (TNR): also called specificity is computed by: $t n /(t n+f p)$

- True Positive Rate (TPR): also called sensitivity or recall is given by: $t p /(t p+f n)$

- Geometric mean (G-mean) (Kubat et al., 1997): $\sqrt{T P R \times T N R}$

- Area under the ROC and Precision-Recall curves: In the specific case of this section, we also consider the Precision-Recall (PR) curve which analyzes the precision versus the recall (see Section 3.5.2). The PR curve can provide additional information in the case of imbalanced datasets as the precision is affected by the class imbalance owing to the computation of the false positives.

where $t p, t n, f p$, and $f n$ stand for true positive, true negative, false positive, and false negative, respectively.

\section{Results in the diagnosis of damaged discs}

The results obtained during the evaluation of the different SCNN models on the test set composed of 245 images are illustrated in Table 3.5. The results presented in Table 3.5 show the mean and standard deviation after conducting 10 experiments on each SCNN configuration and thresholding at 0.5 the output of the sigmoid activation function of the final layer for computing the TNR, TPR and G-mean metrics. The ROC-AUC and PRAUC are computed in the standard way by considering several classification thresholds. As can be seen in Table 3.5, both distance layers (i.e., L1 and Chi-square) provide high performance for diagnosing damaged disc units, being the performance of the networks using the L1 layer greater in most of the configurations analyzed. This can be clearly observed when analyzing the configurations SCNN-L1 $/ \chi^{2}, \mathrm{SCNN}-\mathrm{S} 3-\mathrm{L} 1 / \chi^{2}, \mathrm{SCNN}-\mathrm{C} 4-\mathrm{L} 1 / \chi^{2}$, and SCNN-c4-L1 $/ \chi^{2}$ _vgg 16 , where the relative improvement considering the ROC-AUC and PR-AUC scores respectively is $1.5 \%$ and $1.0 \%$ for the SCNN-L1 $/ \chi^{2}, 1.8 \%$ and $1.7 \%$ for the SCNN-c3-L1 $/ \chi^{2}, 0.8 \%$ and $1.1 \%$ for the SCNN-c4-L1 $/ \chi^{2}$, and $1.7 \%$ and $1.9 \%$ for the SCNN-c4-L1 $/ \chi^{2} \_$vgg16. Another interesting result derived from the results presented in Table 3.5, is the considerable influence of transfer learning. In all the architectures analyzed, the usage of pre-trained weights from the VGG16 model produced an important 
increase of the TPR. This effect can be observed in Table 3.5 when analyzing the results of the configurations SCNN-c3-L1 and SCNN-c4-L1, where the usage of their pre-trained counterparts produced an increment of the TPR of $3.6 \%$ and $1.7 \%$, respectively. This effect is also observed in the case of the SCNN-c3- $\chi^{2}$ and SCNN-c4- $\chi^{2}$ variants, where the increment in the TPR when using a pre-trained model is $6.2 \%$ and $1.3 \%$, respectively. Finally, it should be remarked the effect of adding the $1 \times 1$ convolutional layer (c4 configurations). According to the results presented in Table 3.5, this new architecture has beneficial effects when considering the L1 variant. The transition from SCNN-c3L1 to SCNN-c4-L1 produces an increment of $1.5 \%, 2.4 \%$, and $2.7 \%$ when analyzing the G-mean, ROC-AUC, and PR-AUC respectively. Similarly, The transition from SCNNc3-L1_vgg16 to SCNN-c4-L1_vgg16 causes an increment in G-mean, ROC-AUC, and PR-A $\bar{U}$ C of $2.7 \%, 4.2 \%$, and $7.7 \%$ respectively.

Table 3.5: Mean and standard deviation obtained by averaging the results of 10 different models (10 trainings) for each SCNN configuration while diagnosing damaged disc units on a test set composed of 245 images. Networks whose name ends in "vgg16" are pre-trained with the weights of the VGG16 network trained on ImageNet. The number of parameters of the networks is expressed in millions. Best results are indicated in bold.

\begin{tabular}{|c|c|c|c|c|c|c|}
\hline SCNN conf. & TNR (\%) & $\operatorname{TPR}(\%)$ & G-mean $(\%)$ & ROC-AUC (\%) & PR-AUC (\%) & $\#$ param $(\mathrm{M})$ \\
\hline SCNN-L1 & $86.67 \pm 4.95$ & $92.77 \pm 2.72$ & $89.61 \pm 2.41$ & $93.58 \pm 1.05$ & $92.53 \pm 1.22$ & 1.29 \\
\hline SCNN- $\chi^{2}$ & $87.53 \pm 4.20$ & $91.93 \pm 3.01$ & $89.64 \pm 1.44$ & $92.08 \pm 1.85$ & $91.50 \pm 2.11$ & 1.29 \\
\hline SCNN-c3-L1 & $88.64 \pm 4.10$ & $89.52 \pm 4.33$ & $89.02 \pm 2.69$ & $91.48 \pm 3.57$ & $91.27 \pm 3.06$ & 6.00 \\
\hline SCNN-c3- $\chi^{2}$ & $89.32 \pm 5.13$ & $87.47 \pm 2.74$ & $88.32 \pm 2.03$ & $89.71 \pm 2.32$ & $89.62 \pm 2.53$ & 6.00 \\
\hline SCNN-c3-L1_vgg16 & $86.23 \pm 4.87$ & $93.14 \pm 3.17$ & $89.54 \pm 1.67$ & $92.41 \pm 1.24$ & $88.64 \pm 1.53$ & 6.00 \\
\hline SCNN-c3- $\chi^{2}+\operatorname{vgg} 16$ & $81.98 \pm 2.17$ & $93.62 \pm 2.34$ & $87.59 \pm 1.64$ & $92.50 \pm 2.22$ & $90.57 \pm 2.65$ & 6.00 \\
\hline SCNN-c4-L1 & $88.83 \pm 5.75$ & $92.41 \pm 1.61$ & $90.55 \pm 2.80$ & $93.92 \pm 1.24$ & $93.98 \pm 1.28$ & 2.82 \\
\hline SCNN-c4- $\chi^{2}$ & $82.29 \pm 5.35$ & $92.17 \pm 2.97$ & $87.00 \pm 2.04$ & $93.11 \pm 1.80$ & $92.88 \pm 2.22$ & 2.82 \\
\hline SCNN-c4-L1 vgg16 & $90.49 \pm 4.09$ & $94.10 \pm 1.55$ & $92.25 \pm 1.93$ & $96.59 \pm 1.60$ & $96.36 \pm 1.11$ & 2.82 \\
\hline SCNN-c4- $\chi^{2}+\operatorname{vgg} 16$ & $84.32 \pm 3.26$ & $93.50 \pm 2.14$ & $88.76 \pm 1.30$ & $94.89 \pm 0.83$ & $94.45 \pm 1.00$ & 2.82 \\
\hline SCNN-c4-L1 $\operatorname{vgg} 16 \quad$ SYN & $83.33 \pm 4.83$ & $89.88 \pm 3.69$ & $86.47 \pm 2.03$ & $92.37 \pm 2.68$ & $89.89 \pm 3.54$ & 2.82 \\
\hline SCNN-c4-L1_vgg16_REAL & $87.41 \pm 5.41$ & $60.12 \pm 5.66$ & $72.36 \pm 3.30$ & $71.90 \pm 3.99$ & $73.67 \pm 4.30$ & 2.82 \\
\hline
\end{tabular}

The two final rows of Table 3.5 show the results obtained when training the SCNNc4-L1_vgg16 model using only synthetic or only real defects. Interestingly, training the SCNN-c4-L1_vgg16 model using only synthetic defects provides a G-mean score of $86.47 \%$ which is very close to the performance of other variants of the model trained on the complete dataset (i.e., real plus synthetic defects). On the other hand, training the SCNN-c4-L1_vgg16 model using only real defects allows obtaining a decent performance (G-mean score of $72.36 \%$ ) taking into account that only 18 damaged discs images (95 image pairs) containing real defects were available for training purposes.

Fig. 3.10 shows the ROC and PR curves generated by the best models among the ten trainings conducted on each SCNN configuration. In this case, the best model is selected by taking the one with the highest ROC-AUC score. According to the results presented in Table 3.5 and Fig. 3.10, the SCNN-c4-L1_vgg16 architecture exhibits the best performance in the diagnosis of damaged discs, and is further evaluated in Table 3.6 against its CNN counterpart and the SCAE architectures. In order to properly compare the siamese architectures with the CNN model, since they take different types of inputs 
(i.e., the CNN takes as input a single image, whereas the siamese architectures take as input a pair of images), for each image evaluated on the CNN we extract the corresponding pairs in which this image appears from the SCNN's test set. Then, the probabilities generated by the SCNN are averaged using the corresponding pairs and compared to the probability predicted by the $\mathrm{CNN}$ on the single image.

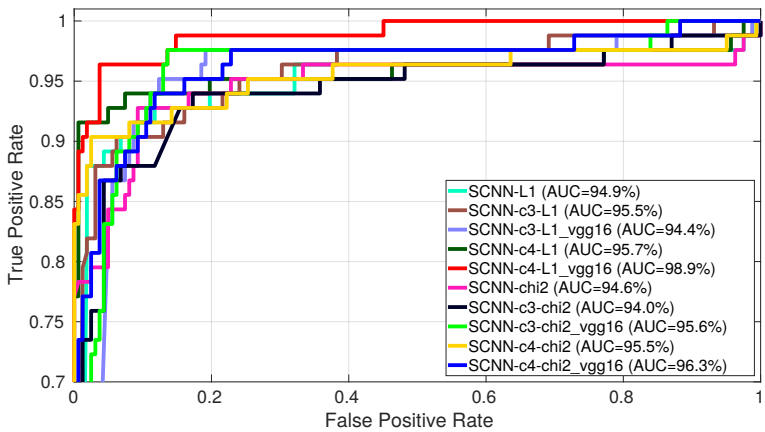

(a)

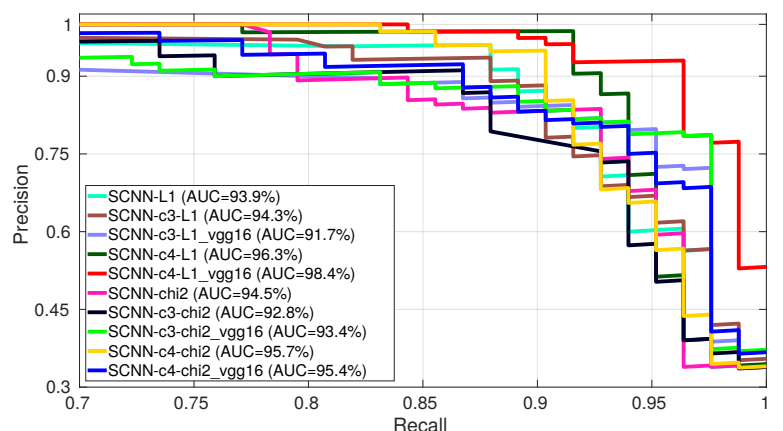

(b)

Figure 3.10: ROC and Precision-Recall curves generated by the best model for each SCNN configuration tested for the diagnosis of damaged disc units (best seen in color). (a) ROC curve depicted using the best model out of 10 for each SCNN configuration. (b) Precision-Recall curve generated by the selected model from Fig. 3.10a. Axes in both figures have been adjusted for visualization purposes.

Table 3.6: Comparison between the supervised and semi-supervised learning frameworks for diagnosing damaged discs, using a test set of 193 disc images. Best results are indicated in bold.

\begin{tabular}{ccccccc}
\hline Model arch. & TNR (\%) & TPR $(\%)$ & G-mean $(\%)$ & ROC-AUC (\%) & PR-AUC (\%) & \#param $(\mathrm{M})$ \\
\hline CNN-c4_vgg16 & $86.69 \pm 2.38$ & $86.83 \pm 2.77$ & $86.74 \pm 1.73$ & $91.84 \pm 0.83$ & $89.28 \pm 2.16$ & 2.82 \\
SCNN-c4-L1_vgg16 & $86.56 \pm 3.93$ & $\mathbf{9 0 . 4 2} \pm \mathbf{3 . 1 8}$ & $88.42 \pm 1.64$ & $\mathbf{9 3 . 6 7} \pm \mathbf{2 . 0 3}$ & $\mathbf{9 2 . 7 5} \pm \mathbf{1 . 7 0}$ & 2.82 \\
SCAE-c4-L1_OC_SVM & $91.73 \pm 0.00$ & $78.33 \pm 0.00$ & $84.77 \pm 0.00$ & $85.03 \pm 0.00$ & $83.05 \pm 0.00$ & 2.93 \\
SCAE-c5-L1_OC_SVM & $\mathbf{9 5 . 4 9} \pm \mathbf{0 . 0 0}$ & $83.33 \pm 0.00$ & $\mathbf{8 9 . 2 0} \pm \mathbf{0 . 0 0}$ & $89.41 \pm 0.00$ & $88.90 \pm 0.00$ & 13.26 \\
\hline
\end{tabular}

The result of this comparison is summarized in Table 3.6, which shows how the SCNNc4-L1_vgg16 outperforms the CNN-c4_vgg16 by $1.8 \%$ and $3.5 \%$ in ROC-AUC and PRAUC, respectively. Furthermore, the SCNN-c4-L1_vgg16 exhibits an improvement of $3.6 \%$ when analyzing the TPR. Regarding the SCAE architectures, despite being trained only on non-damaged disc examples, they provide very high performance, highlighting the results of the SCAE-c5-L1_OC_SVM, which is the model that provides the highest TNR and G-mean scores. With respect to AUC metrics, which are the most representative for selecting the appropriate model, the proposed SCAE-c5-L1_OC_SVM obtains a very high score with a ROC-AUC of $89.4 \%$ and a PR-AUC of $88.9 \%$, again noting that the SCAE architectures have only been trained on healthy disc images.

Taking into account the results presented in Table 3.5 and 3.6, the SCNN-c4-L1_vgg16 architecture is selected as the final model for diagnosing damaged disc units in the final system. Fig. 3.11 shows the predictions generated by the selected SCNN-c4-L1_vgg16 in 
some representative examples extracted from the test set. As compared to the training set, the test set contains a more heterogeneous set of defective images in terms of the type of material and the type of defect. As shown in Fig. 3.11b, the test set encompasses images of disc units made of different material such as glass or ceramic, which can be burned, polluted (with a small to severe degree of pollution), or present rust on their surface. Furthermore, within each defect type, there exists considerable variability in the appearance of the defect on the surface of the disc, and even some discs can show more than one type of defect. The high performance of the proposed SCNN-c4-L1_vgg16 for the diagnosis of the aforementioned types of defects reveals the good generalization capabilities of this model, which are confirmed in the results presented in Table 3.7 for several video sequences related to inspections of high voltage power transmission lines.
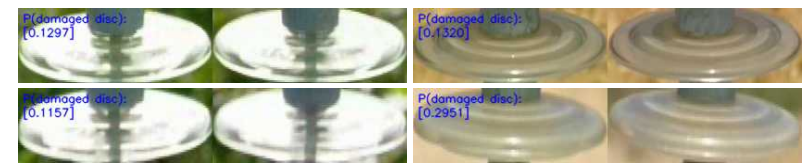

(a)
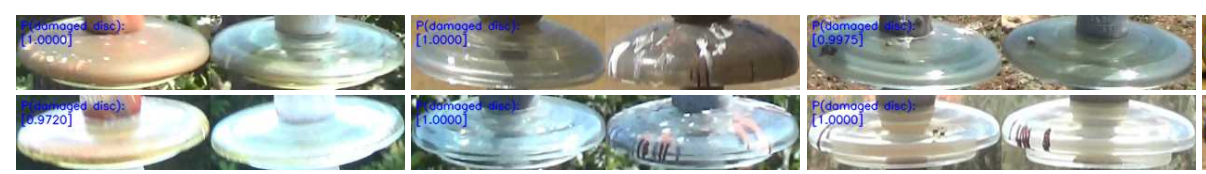

(b)

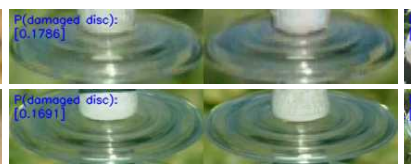

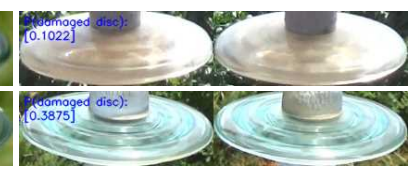

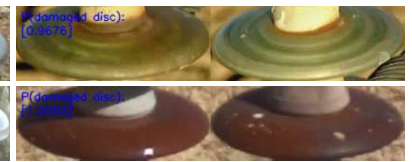

Figure 3.11: Examples of disc images captured in different inspections of high voltage power lines, extracted from the test set used for evaluating the SCNN models. (a) Non-damaged examples and probabilities predicted by the SCNN-c4-L1_vgg16. (b) Damaged examples and probabilities predicted by the SCNN-c4-L1_vgg16. Defects of varying nature such as discs burned, polluted (with different degrees of pollution), and rusted are efficiently detected by the proposed SCNN.

\subsubsection{System evaluation results}

The objective of this section is to perform a thorough evaluation of the complete system (see Section 3.3) proposed in this work. To this aim, we integrate all the previously evaluated components and evaluate them on several video sequences captured during real aerial inspections of overhead power line infrastructure. Our proposed system has been evaluated in 5 video sequences corresponding to inspections of high voltage power lines (see Table 3.7), comprising a total of 5806 frames of $720 \times 576$ pixels, which have not been used in the training process of any of the components of the system. Sequences 1 to 4 contain high voltage insulator strings with different types of defects, sequence 1 being the most representative as it comprises defects of varying nature such as discs with bird excrements, with rust, etc. As stated previously in this chapter, we include all these types of defects within the "damaged" category for convenience. Sequence 5 contains four high voltage insulator strings, which do not present any defect. However, this sequence is of particular interest since two insulator strings are in a vertical position, and the 
other two are tilted, thus allowing the validation of the proposed system to changes in the orientation of the insulator string. All the sequences have been manually annotated, where each frame has been classified as defective or not by a human inspector.

Table 3.7: Results obtained in the evaluation of the proposed diagnosis system using five video sequences of real aerial inspections. The processing time represents the total time taken by the proposed system for processing each video sequence. The average diagnosis time considers each diagnosis component independently.

\begin{tabular}{|c|c|c|c|c|c|c|c|}
\hline Sequence & \#frames & $\begin{array}{c}\# \text { insulator } \\
\text { strings }\end{array}$ & $\begin{array}{c}\text { processing } \\
\text { time }(\mathrm{s})\end{array}$ & $\begin{array}{c}\text { diagnosis } \\
\text { component }\end{array}$ & $\begin{array}{l}\text { \#diagnosed } \\
\text { frames }\end{array}$ & $\operatorname{accuracy}(\%)$ & $\begin{array}{c}\text { avg. diagnosis } \\
\text { time (ms/frame) }\end{array}$ \\
\hline \multirow{2}{*}{ Seq1 } & \multirow{2}{*}{1700} & \multirow{2}{*}{3} & \multirow{2}{*}{50.57} & damaged disc & 565 & 96.64 & 15.93 \\
\hline & & & & absent disc & 732 & 99.45 & 2.87 \\
\hline \multirow{2}{*}{ Seq2 } & \multirow{2}{*}{1376} & \multirow{2}{*}{3} & \multirow{2}{*}{41.90} & damaged disc & 386 & 93.26 & 19.09 \\
\hline & & & & absent disc & 537 & 99.81 & 2.88 \\
\hline \multirow{2}{*}{ Seq3 } & \multirow{2}{*}{1213} & \multirow{2}{*}{3} & \multirow{2}{*}{38.16} & damaged disc & 400 & 98.75 & 18.13 \\
\hline & & & & absent disc & 537 & 100 & 2.85 \\
\hline \multirow{2}{*}{ Seq4 } & \multirow{2}{*}{487} & \multirow{2}{*}{2} & \multirow{2}{*}{20.04} & damaged disc & 279 & 88.89 & 23.17 \\
\hline & & & & absent disc & 348 & 100 & 2.87 \\
\hline \multirow{2}{*}{ Seq5 } & \multirow{2}{*}{1030} & \multirow{2}{*}{4} & \multirow{2}{*}{31.95} & damaged disc & 205 & 100 & 32.88 \\
\hline & & & & absent disc & 205 & 97.56 & 2.88 \\
\hline
\end{tabular}

Table 3.8: Results of the disc absent fault diagnosis on the simulated sequences shown in Fig. 3.13. The processing time represents the total time taken by the proposed system for processing each video sequence, disabling the damaged disc diagnosis component.

\begin{tabular}{cccccccc}
\hline Sequence & \#frames & $\begin{array}{c}\text { \#insulator } \\
\text { strings }\end{array}$ & $\begin{array}{c}\text { processing } \\
\text { time (s) }\end{array}$ & $\begin{array}{c}\text { diagnosis } \\
\text { component }\end{array}$ & $\begin{array}{c}\text { \#diagnosed } \\
\text { frames }\end{array}$ & accuracy(\%) & $\begin{array}{c}\text { avg. diagnosis } \\
\text { time (ms/frame) }\end{array}$ \\
\hline Seq1 & 1700 & 3 & 44.58 & absent disc & 804 & 99.25 & 2.81 \\
Seq2 & 1213 & 3 & 33.42 & absent disc & 600 & 98.33 & 2.83 \\
\hline
\end{tabular}

Regarding the absent disc defect, none of the five test sequences present any defect of this type. Thus, in order to further evaluate the disc absent diagnosis component, two additional sequences have been generated (see Table 3.8) where we simulate the absence of disc insulator units in each insulator string by alternatively removing disc units in the mask generated by the insulator string segmentation component. In order to automate the process for removing disc units within the masks, a computer program has been implemented, which takes as input a correct segmentation image, and uses image processing algorithms (contour extraction, contour approximation, and drawing functions) to alternatively remove disc units. The disc or discs to be removed for a given image are indicated to the program by manually clicking on the designated disc.

The results obtained for each diagnosis component on the video sequences of real inspections are shown in Table 3.7. Similarly, the results obtained for the disc absent diagnosis component on the simulated sequences are shown in Table 3.8. In both tables, the accuracy represents the performance of each diagnosis component for classifying each 
frame as defective or not, taking as ground truth the labels annotated by a human inspector. As can be seen in Table 3.7, the accuracy of the system is above $88.9 \%$ and $97.6 \%$ for the damaged-disc and absent-disc diagnosis components respectively in all the sequences.

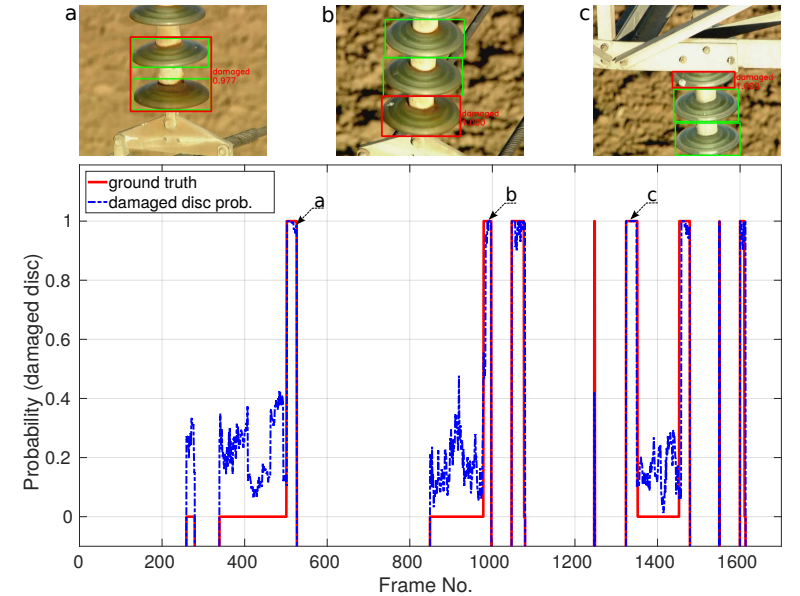

(a)

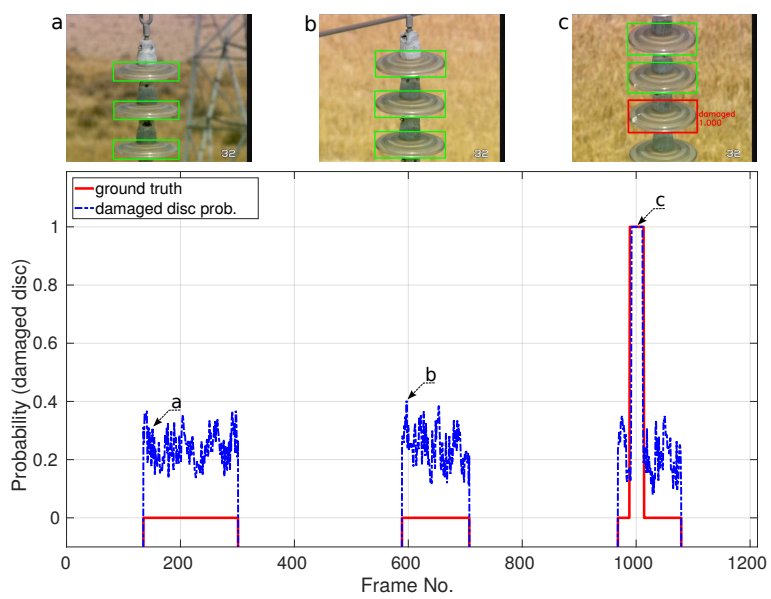

(c)

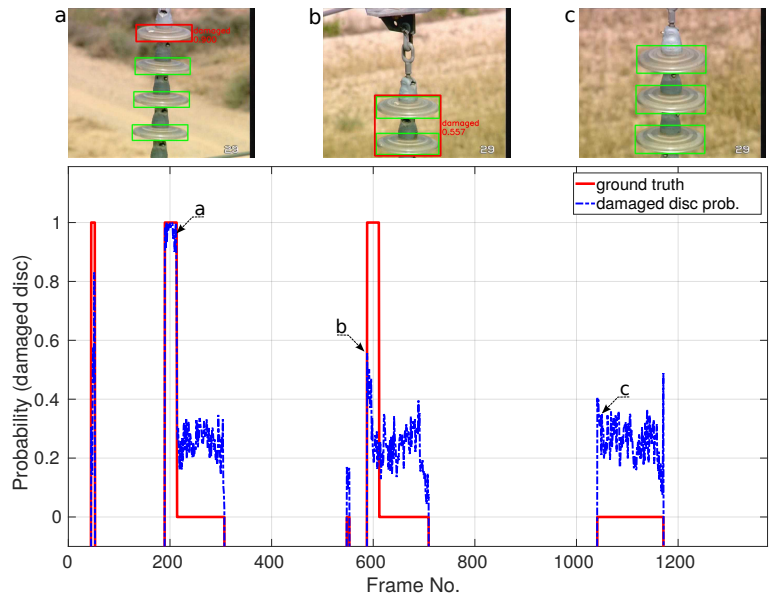

(b)

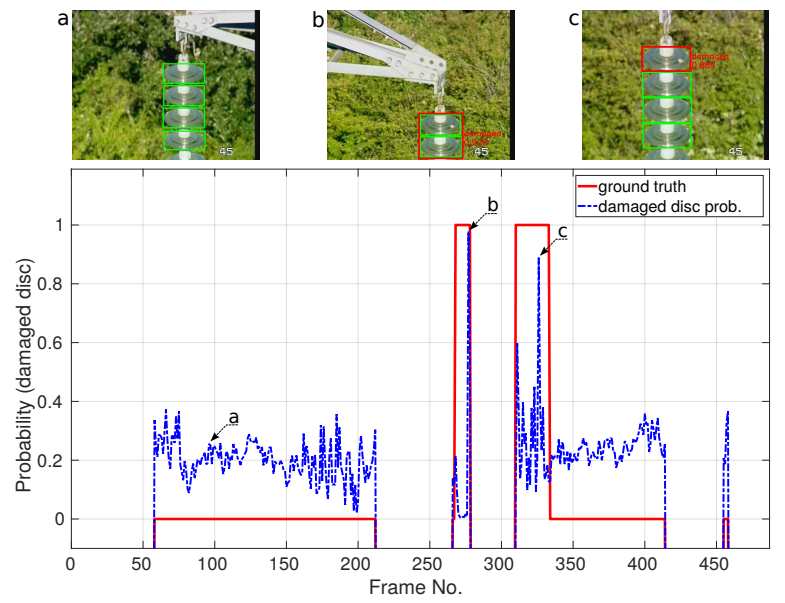

(d)

Figure 3.12: Results obtained by the damaged disc diagnosis component in four test video sequences corresponding to real aerial inspections of high voltage power transmission lines (best seen in color). (a) to (d) correspond to sequences 1 to 4 in Table 3.7. Taking into account the output provided by the insulator string segmentation component, some of the frames are automatically discarded (not diagnosed) by our system when the insulator string does not contain the sufficient number of discs.

In order to properly understand the results presented in Table 3.7, in Fig. 3.12 we show the average probability generated by the damaged disc diagnosis component on each frame of the sequences. This probability is calculated by averaging the probabilities of all the disc pairs combinations when the criteria for considering a disc damaged are not satisfied, and averaging the probabilities of the positive disc pairs (those combinations with a probability given by the SCNN-c4-L1_vgg16 model greater than a threshold) when 
the defect criteria are satisfied. As can be noticed in Fig. 3.12a and 3.12c, sequences 1 and 3 contain prominent defects which are correctly identified by our proposed system with a high probability in almost every frame in which they appear. This leads to a very low false negative rate which is translated into the high accuracy obtained for sequences 1 and 3 (96.6\% and $98.8 \%$ respectively).

On the other hand, Fig. $3.12 \mathrm{~b}$ and $3.12 \mathrm{~d}$ show the average probabilities given by the damaged disc diagnosis component on sequences 2 and 4, respectively. As can be seen in these figures, sequences 2 and 4 present small defects mainly due to bird excrements which are correctly detected by our system. The second insulator in sequence 2 (frames 548 to 709 in Fig. 3.12b has a very small defect annotated by the human inspector which is detected by our system with a probability greater than 0.5 in only two frames of the sequence. Similarly, the second insulator in sequence 4 (frames 266 to 458 in Fig. $3.12 \mathrm{~d}$ ) has a small defect which in addition is blurred due to the camera movement. Despite this fact, our system is capable of detecting this defect with a probability greater than 0.5 in four frames of the sequence. The fact of detecting the defect in only a few frames increases the false negative rate in detriment of the accuracy, which is $93.3 \%$ and $88.9 \%$ for sequences 2 and 4, respectively. Despite obtaining few detections in some sequences, due to the aforementioned constraints, we want to remark that the overall performance of the damaged disc diagnosis component, when considering the number of detected defects, is greater than the accuracy presented in Table 3.7 since only one frame is enough to consider one defect as detected.

Another important aspect to take into account when analyzing the results presented in Table 3.7 and Fig. 3.12 are the criteria adopted for considering the current frame as defective in terms of damaged discs. The criteria followed by the experiments presented in this section are explained in Algorithm 2 and consider a frame as defective if there is unanimity of all the disc image pairs in the current frame. As an example, if we consider the first insulator string in sequence 2 (frames 45 to 307 in Fig. 3.12b), frame 208 (see frame $a$ in Fig. 3.12b) is marked as defective, and the defect on the first disc is located in the image, since three out of the four disc units are voting for having a defect when combined with the first disc of the insulator string.

Regarding the evaluation of the absent disc diagnosis component on the simulated sequences shown in Table 3.8, in Fig. 3.13 we illustrate the probabilities generated by the absent disc diagnosis component on each frame of the sequences. The results presented in Fig. 3.13 show the low false positive and false negative rates of the proposed system. As shown in Fig. 3.13a, different scenarios for the disc absent defect have been simulated, where we alternately remove one or more disc units. In the latter case (see frame $b$ in Fig. 3.13a where several discs are missing in the insulator string, the proposed system exhibits robust diagnosis capabilities, which lead to a classification accuracy of $99.3 \%$ for sequence 1. Similarly, Fig. 3.13b depicts the probabilities generated by the absent disc diagnosis component in sequence 2. As shown in Fig. 3.13b, the system is able to correctly detect the absence of disc insulator units even when the segmented insulator string occupies a small area in the image (see frame $c$ in Fig. 3.13b), leading to an accuracy of $98.3 \%$. 


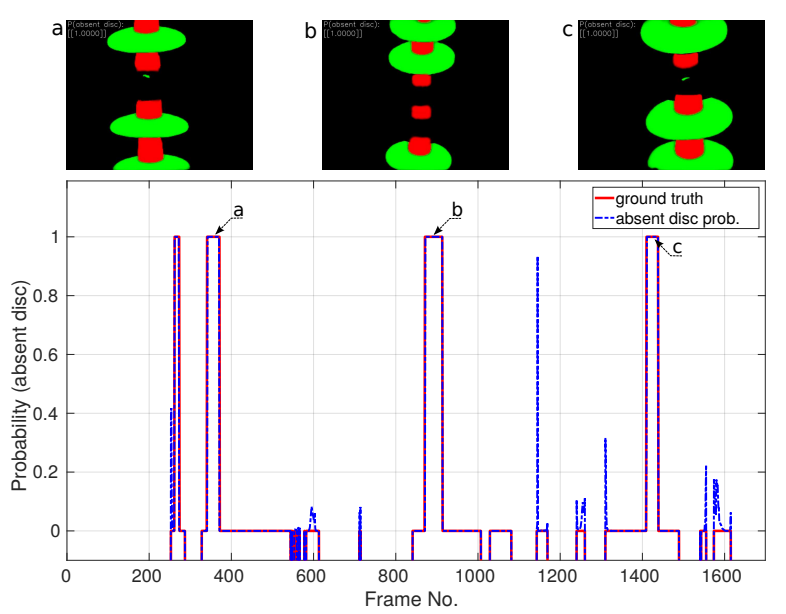

(a)

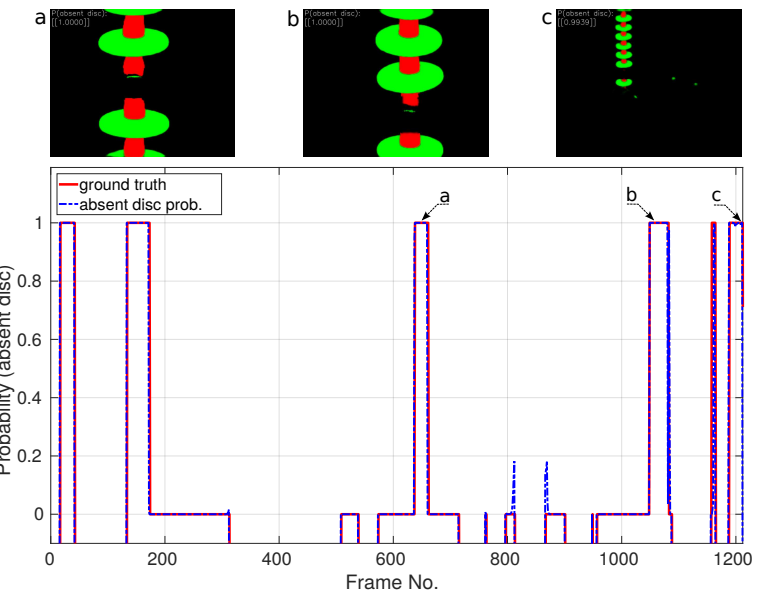

(b)

Figure 3.13: Results obtained by the absent disc diagnosis component in two simulated sequences (best seen in color). (a), (b) correspond to sequences 1 and 2 in Table 3.8, respectively. The absence of disc units is simulated by alternatively removing discs from the mask generated by the insulator string segmentation component. Taking into account this mask, our system automatically discards some of the frames when they do not contain an insulator string for being diagnosed.

\subsection{Discussion}

The results presented throughout this chapter show the outstanding results provided by supervised learning techniques when having enough training examples for the anomalous class. To this aim, synthetic defect generation has demonstrated to be an effective solution. All the proposed CNN models for diagnosing the absence of disc insulator units have been trained using only synthetic masks simulating the absence of discs in the segmentation masks generated by the insulator string segmentation component. The results presented in Table 3.4, reveal the importance of three main factors: the number of convolutional layers, the size of the convolution kernels, and the down-sampling factor. Regarding the number of convolutional layers, the effect of adding one more convolutional layer in the second and third convolutional blocks, that is, when considering the transition from CNN1 to CNN3, provides an improvement in ROC-AUC of $1.6 \%, 0.6 \%$, and $0.9 \%$ using kernels of size $3 \times 3,5 \times 5$, and $7 \times 7$, respectively. Increasing the size of the convolution kernels, that is, considering the transition from $3 \times 3$ to $7 \times 7$ produces an increment in ROC-AUC of $1.9 \%, 0.6 \%$, and $1.2 \%$ for CNN1, CNN2, and CNN3, respectively. Finally, the effect of the down-sampling factor when conducting a forward pass to the different CNNs is of special interest. More concretely, the proposed CNN2 has an additional down-sampling stage as compared to CNN1 and CNN3, due to the stride of 2 applied in the first convolutional layer of the second convolutional block. In total, CNN2 applies a $\times 32$ down-sampling factor which allows reducing the number of parameters of the network by a large margin. Despite having much less number of parameters, for example when considering CNN2_5x5 which has 5 times less number of parameters (i.e., 0.56 
M) as compared to its CNN3_5x5 counterpart (i.e., 2.61 M), or CNN2_7x7 which has 3.7 times less number of parameters (i.e., $0.91 \mathrm{M}$ ) as compared to CNN3_7x7 (i.e., $3.35 \mathrm{M}$ ), the performance of the CNN2 model is very similar to the final selected CNN3. This fact can be produced owing to the type of inputs used in the proposed strategy. The input to the CNN model for diagnosing the absence of disc units consists of a segmentation mask, and thus down-sampling operations can be more beneficial than training these models using RGB images. Based on the previous analysis, CNN2 is considered as an alternative candidate in systems with severe computational constraints.

Regarding the diagnosis of damaged disc units, the results presented in Table 3.5 again reveal the beneficial effect of using synthetic samples for training within the supervised learning framework. This fact can be observed when analyzing the results presented in the last two rows of Table 3.5. Training the SCNN-c4-L1_vgg16 model using only synthetic defects provides an increment of $20.5 \%$ and $16.2 \%$ in ROC-AUC and PR-AUC, respectively, as compared to training the same model using only real defects. Training the SCNN-c4-L1_vgg16 using both synthetic and real defects allows obtaining a ROC-AUC and PR-AUC of $96.6 \%$ and $96.4 \%$ which drives to an increment of $4.2 \%$ and $6.5 \%$ in ROCAUC and PR-AUC, respectively, as compared to the model trained using only synthetic defects. Another important factor related to the training process within the supervised learning framework when relatively few training examples of real defects are available, is the influence of transfer learning. As shown in Table 3.5 for the models SCNN-c4-L1/ $\chi^{2}$, the transition to their analogous pre-trained models produces an increment in ROC-AUC and PR-AUC of $2.7 \%$ and 2.4\%, respectively, for the SCNN-c4-L1, and $1.8 \%$ and $1.6 \%$, respectively, for the SCNN-c4- $\chi^{2}$. Transferring the weights from a VGG16 model trained on ImageNet has proven to be an appropriate initial condition for the training process of the SCNNs.

Two additional factors that condition the performance of the SCNN models for diagnosing damaged discs are the energy function used for extracting the encodings and the size of the SCNN. Regarding the energy function, the results presented in Table 3.5 demonstrate the beneficial effects of using an L1 distance layer as compared to the Chi-squared. In most of the network architectures presented in Table 3.5, the L1 layer outperforms its $\chi^{2}$ counterpart. This can be observed when analyzing the SCNN-c4-L1/ $\chi^{2}$ architecture, where the improvement in ROC-AUC and PR-AUC when using the L1 is $1.7 \%$ and $1.9 \%$, respectively. Regarding the size of the SCNN, it should be noted the beneficial effect of adding a fourth convolutional block consisting of a $1 \times 1$ convolution. The SCNNc4-L1_vgg16 is benefited by its fourth convolutional block (conv4), which implements a $1 \times 1$ convolutional layer as a similar concept of the "Network in Network" proposed in Lin et al., 2013, with a double purpose of shrinking the number of channels before reaching the fully-connected part, providing at the same time another complex level of abstraction to the SCNN. This improvement in performance when adding the fourth convolutional block can be observed when analyzing the transition from the SCNN-c3-L1/ $\chi^{2}$ to the SCNN-c4-L1 $/ \chi^{2}$. The transition from SCNN-c3-L1 to the SCNN-c4-L1 model drives to an improvement in ROC-AUC and PR-AUC of $4.2 \%$ and $7.7 \%$, respectively. The transition from SCNN-c3- $\chi^{2}$ to the SCNN-c4- $\chi^{2}$ model produces an increment in ROC-AUC and PR-AUC of $2.4 \%$ and $3.9 \%$, respectively. Shrinking the number of channels produced 
when adding the $1 \times 1$ convolution has also an important effect in reducing the number of parameters of the network, where the model SCNN-c4-L1/ $\chi^{2}$ has half the number of parameters than the SCNN-c3-L1/ $\chi^{2}$.

Regarding the semi-supervised learning framework, it should be remarked the high performance obtained by the proposed model based on the combination of an SCAE and OC-SVM, taking into account that these models have only been trained using normal examples. The semi-supervised learning framework presented in this chapter has demonstrated outstanding capabilities in diagnosing damaged discs, providing a ROC-AUC and PR-AUC of $89.4 \%$ and $88.9 \%$, respectively, in the test set used in Table 3.6. In this regard, the proposed SCAE-c5-L1_OC_SVM provides competitive results as compared to the best diagnosis model (SCNN-c4-L1_vgg16), having only a difference of $4.3 \%$ and $3.9 \%$ in ROC-AUC and PR-AUC, respectively. Thus, the proposed SCAE_OC_SVM represents an appropriate solution when no training data related to the anomalous class are available. The good performance exhibited by the SCAE-c5-L1_OC_SVM, is benefited by the use of a siamese architecture in combination with an OC-SVM. Using only the CAE-c5 network as a discriminative model, what implies the usage of the reconstruction error of the autoencoder as a classification threshold, allows obtaining a ROC-AUC and PR-AUC of $71.1 \%$ and $66.3 \%$, respectively.

Finally, the results presented in Tables 3.7 and 3.8 reveal the effective combination of the different learning-based components to build a complete system for the automatic detection and diagnosis of electrical insulator strings. The results presented in Table 3.7 . show that the performance of the proposed diagnosis system is always above $89 \%$ and $97 \%$ when considering the damaged-disc and absent-disc diagnosis components, respectively. It should be noted, that this performance is obtained at frame-level, that is, frames correctly classified as containing a defective element or not. Overall, the accuracy of the proposed diagnosis system when considering the number of defects that are contained in the video sequences is $100 \%$, meaning that the system correctly identifies all the defects. Thus, the proposed system has demonstrated to be an efficient solution to reduce the workload of human inspectors, who in current production systems need to review frame by frame the collected inspection videos.

\subsection{Conclusions}

In this chapter, a complete system for automatic diagnosis of anomalies in electrical insulator strings using RGB images has been proposed. Anomalies in these elements are mainly due to the absence of disc units and the presence of damaged discs. The latter category presents a considerable variability regarding the type of defect (e.g., disc polluted, burned, rusted, etc.) and the variants that can appear per type of defect (e.g., varying degree of pollution and rust on the surface of the disc).

The diagnosis of absent disc units has been addressed in this work by using a supervised learning framework consisting of a 10-layer CNN which is trained using only synthetic masks. This strategy has demonstrated to provide several advantages which are summarized next: 
- The proposed CNN takes as input the entire segmented image, so no additional algorithms for the location of the insulator string in the image are required. This simplifies the implementation while maintaining the modularity of the system.

- Since the proposed CNN takes segmentation masks as input, it can be trained using synthetic masks without the need to collect more training data corresponding to real RGB images. This strategy makes the proposed approach extremely flexible to be adapted to new types of insulators and inspections that can be conducted in future works, since a large number of training examples simulating different defect configurations can be synthetically generated.

- Since the computational cost derived from the inference to the CNN is minimal (2.8 ms approximately on an Nvidia GeForce GTX 1080Ti GPU), the proposed approach can always be utilized in conjunction with other alternative solutions proposed in future works. Furthermore, the performance of the proposed CNN (greater than $98 \%$ in the test set, and $97 \%$ in all the test video sequences) demonstrate the generalization capabilities and the robustness of the proposed CNN.

The diagnosis of damaged disc units, that is, the diagnosis of anomalies that can appear on the surface of disc insulator units, has been addressed in this chapter by a novel strategy which integrates Siamese Convolutional Neural Networks responsible for modeling the similarity between adjacent disc units. Several variants of the siamese networks have been designed within a supervised and a semi-supervised learning frameworks providing outstanding diagnosis capabilities. The main advantages and findings using the proposed architectures are summarized next:

- The proposed siamese architecture in its supervised and semi-supervised variants has demonstrated outstanding capabilities for anomaly detection. The SCNNs and SCAEs presented in this chapter have shown to be very effective to handle the enormous variability concerning the types of defects when very few training data relative to the defects are available.

- Synthetic defect generation has demonstrated to be an effective solution to increase the training data. In the diagnosis of damaged discs, 125 images containing synthetic defects were added to the original dataset, leading to a considerable increment in the diagnosis performance. The generation of synthetic defects has been manually conducted in this work, and their automatic generation using generative models such as GANs will be considered for future works.

- The final proposed model for diagnosing damaged disc units, consisting of an SCNN architecture composed of two 13-layer twin CNNs joined by a weighted L1 layer, has shown to be benefited by different deep learning techniques. The results obtained in the evaluation of different SCNN architectures reveal the beneficial effect of integrating a $1 \times 1$ convolutional layer before the fully-connected part of each twin CNN of the SCNN, which helps in reducing the number of parameters of the network while providing an additional level of abstraction. In addition, the integration of an 
L1 distance layer has shown to provide better diagnosis capabilities as compared to other distance layers (e.g., Chi-squared). Added to this is the positive effect of performing transfer learning using the weights of the VGG16 model previously trained on the ImageNet dataset. The final selected model for diagnosing damaged discs (SCNN-c4-L1_vgg16) obtained diagnostic accuracies above $89 \%$ in all the evaluated sequences, reaching $100 \%$ in some cases.

- Finally, the usage of Siamese Convolutional Autoencoders in combination with OneClass SVM when no training data relative to the anomalous examples are available, has proven to be an effective solution, providing diagnosis performances above $89 \%$ in the test set composed of 193 disc images.

Overall, the usage of synthetic training examples for increasing the number of examples in the anomalous class, the use of transfer learning, the design of siamese architectures, and the combination of the latter with OC-SVM when no training data of anomalies are available, have proven to be very effective solutions for anomaly detection. 



\section{Chapter 4}

\section{Image-Based Visual Servoing}

\subsection{Introduction}

Visual servoing is a mature technique, which has been extensively studied in the literature over the years. Despite many efforts, the classic approach to map visual errors to actuator commands still has limitations in terms of convergence, stability, and gain design. In the field of aerial robotics, visual servoing has been used in a wide range of applications such as object following (Olivares Mendez et al., 2011; Mondragón et al., 2011; Pestana et al., 2013; Thomas et al., 2017; Li et al., 2017), object grasping and manipulation (Thomas et al., 2014; Lippiello et al., 2016), landing (Lee et al., 2012), and inspection tasks (Metni and Hamel, 2007; Sa et al., 2014, Araar and Aouf, 2014), among others. In many cases, technique success has been achieved by linearizing the states around an operating point.

In its two variations, traditional visual servoing strategies, i.e., Position-Based Visual Servoing (PBVS) and Image-Based Visual Servoing (IBVS) have achieved acceptable performance, however notable convergence and stability problems (Espiau, 1994; Chaumette, 1998) can appear if the task is performed in a zone away from the operating point (or linearization point). Some of these problems are related to the calculation of the image Jacobian, also called interaction matrix, which defines a crucial role in the implementation of the control law, but also in the choice of an appropriate gain that provides a good tradeoff between convergence and stability. Other important problems are those related to local minima solutions owing to unrealizable image motions. Moreover, the tuning process of the different parameters of the control model can become an onerous and hard procedure in most of the applications.

Inspired by the aforementioned limitations, in this chapter we present a novel IBVS controller, termed Image-Based Visual Servoing through Reinforcement Learning or RLIBVS (Sampedro et al., 2018b; Sampedro et al., 2018c) for short, which is based on a recent deep reinforcement learning algorithm named Deep Deterministic Policy Gradients (DDPG) (Lillicrap et al., 2015). Taking as input the errors in the image plane with respect to a predefined reference, the agent is successfully trained in order to generate the appropriate linear velocity commands for controlling the aerial robot. We explore different configurations of the proposed RL-IBVS for controlling the aerial robot in 2D (i.e., 
commanding the aerial robot using linear velocities in $x$ and $y$ ) and 3D (i.e., commanding the aerial robot using linear velocities in $x, y$, and $z$ ). In both cases, the proposed RL-IBVS controller acts as a high-level controller which sends velocity commands to the velocity controller integrated into the Aerostack architecture (Pestana et al., 2014).

The training process of the agent has been conducted using our own reinforcement learning framework, which integrates a deep reinforcement learning agent (e.g., DDPG) with an environment based on an aerial robotics simulator (i.e., the RotorS Gazebo proposed in Furrer et al., 2016). In order to infer velocity commands, the agent's state is composed of the errors in the image plane and their respective derivatives. Furthermore, the reward function is carefully designed in order to obtain a smooth behavior of the agent while performing object following missions. These missions have been conducted in simulated and real flight experiments and using different aerial robotic platforms, demonstrating the appropriate capabilities of the proposed RL-IBVS algorithm.

\subsection{Related Work}

The aim of this section is to provide a general overview of recent literature regarding the specific topic of visual servoing with special interest on those approaches which integrate learning-based techniques.

Recent literature on visual servoing and machine learning has focused on learning features and predictive models under the impression that traditional visual servoing has directed most efforts at designing stable and convergent controllers (assuming such features are available). In this chapter, we address a different concept, nowadays there is a suite of robust descriptors and detectors that can work under a wide range of conditions (e.g., complex background, lighting conditions, occlusions, etc.), therefore a problem still remains on designing controllers that are robust and stable in a large task space (not just about the linearization point).

The problem becomes amplified when we consider aerial platforms such as multirotors. One of the pioneering works on the use of reinforcement learning for aerial robots is Abbeel et al., 2007, where a reinforcement learning (optimal control) algorithm based on Differential Dynamic Programming (DDP) was used to find a controller that is optimized for specific aerial maneuvers (forward flip, sideways roll, tail-in funnel, and nose-in funnel). Recent efforts on deep learning and visual servoing include Lee et al., 2017 in which a fitted Q-iteration algorithm is used to learn the weights for the most relevant visual features in object-following missions, however ultimately their optimization function is solved efficiently by the linearization of the dynamics around an operating point. Convolutional Neural Networks (CNNs) have been popular in many areas of robotics, science, and engineering. For instance, in Bateux et al., 2017 authors address the visual servoing problem by training a $\mathrm{CNN}$ to estimate the relative pose between the current and reference image, then having this relative translation and rotation a classic visual servoing control law is used (Chaumette and Hutchinson, 2006b). Authors in Saxena et al., 2017 similarly approach the problem using CNNs as well, however additional experimentation is conducted on a quadrotor using a standard servoing control approach. As compared to previous CNN-based approaches, where the visual servoing algorithm in- 
tegrates the feature extraction stage, our proposed method takes as input the Region Of Interest (ROI) provided by an external object detector algorithm. That is, we decouple the feature extraction (or object detection) stage from the visual servoing task, making it more flexible for being adapted to different IBVS applications.

In terms of control and navigation applied to multirotor aerial robots, reinforcement learning has also been used to learn navigation routines for a quadrotor (Imanberdiyev et al., 2016), control and stabilization (Hwangbo et al., 2017), target tracking (Choi et al., 2017) and landing (Polvara et al., 2017; Rodriguez-Ramos et al., 2018; Rodriguez-Ramos et al., 2019). The closest work to the one presented here is Shi et al., 2016 in which two gains (translation and rotation) are learned to position a quadrotor relative to a target using fuzzy Q-learning. From previous literature, little attention has been placed on learning gains for robustness and stability. This is a standing issue in many decoupled IBVS approaches for aerial robotics.

\subsection{Image-Based Visual Servoing Controllers}

In this section, we describe the theoretical foundations and implementation details of the classic IBVS baselines and the proposed RL-IBVS controllers.

\subsubsection{Classic and Partitioned IBVS Controllers}

The use of IBVS approaches with aerial robots is a challenging task. Classic IBVS as proposed in the literature (Chaumette and Hutchinson, 2006b) establishes that: $\nu=$ $-\lambda \widehat{L_{s}^{+}} e$ will drive linear and angular velocity references $\nu$ to zero as the features $s$ move towards their desired locations $s^{*}$, being the error: $e=s-s^{*}$. This approach requires a minimum of four points, but due to the estimation of varying $3 \mathrm{D}$ parameters during the visual servo, the term $\widehat{L_{s}^{+}}$can be difficult to estimate or approximate. An alternative approach detailed in Malis, 2004, which has proven to provide better convergence capabilities, proposes the approximation of the interaction matrix using the expression $\widehat{L_{s}^{+}}=\frac{1}{2}\left(L_{s}+L_{s^{*}}\right)^{+}$where $L_{s^{*}}$ is the interaction matrix at the desired feature location. In this thesis, we use the approach described above and detailed in Chaumette and Hutchinson, 2006b as one of the baselines techniques (referred to as classic IBVS) to compare the performance of our proposed RL-IBVS controller. Furthermore, we enhance the classic IBVS by introducing an adaptive gain $\lambda(x)=a \exp (-b x)+c$, where $a=\lambda(0)-\lambda(\infty)$, $b=\dot{\lambda}(0) / a$ and $c=\lambda(\infty)$ are constant parameters and $x$ is the infinity norm of the task Jacobian to consider. This controller is named classic adaptive IBVS for convenience.

For aerial vehicles, a common way to deal with underactuated systems such as quadrotors is to decouple the visual servoing task (Corke and Hutchinson, 2001). A decoupled IBVS approach aims at separating translational and rotational degrees of freedom in the control task. A detailed overview of this technique is presented in Chaumette and Hutchinson, 2007. Inspired by the decoupled approaches, the partitioned IBVS emerges (Corke and Hutchinson, 2001; Chaumette and Hutchinson, 2007), which exhibits similar decoupling properties but using only features expressed in the image plane. The formulation of the partitioned IBVS is based on the decoupling of the motion related to the $z$ axis from 
the $x$ and $y$ axes motion. For this purpose, the interaction matrix is partitioned such that:

$$
\begin{aligned}
\dot{s} & =L_{s} \nu \\
& =L_{x y} \nu_{x y}+L_{z} \nu_{z} \\
& =\dot{s}_{x y}+\dot{s}_{z}
\end{aligned}
$$

where $L_{x y}=$ columns $\{1,2,4,5\}$ of $L_{s}$, and $L_{z}=$ columns $\{3,6\}$ of $L_{s}$. The linear and angular velocities of the camera defined by $\nu=(v, \omega)=\left(v_{x}, v_{y}, v_{z}, \omega_{x}, \omega_{y}, \omega_{z}\right) \in \Re^{6}$, are decoupled providing: $\nu_{x y}=\left(v_{x}, v_{y}, \omega_{x}, \omega_{y}\right)$, and $\nu_{z}=\left(v_{z}, \omega_{z}\right)$. The feature velocities in the image are denoted by $\dot{s}$, whose coordinates are defined by $s=(\hat{u}, \hat{v})$ with their desired values being $s^{*}=\left(\hat{u}^{*}, \hat{v}^{*}\right)$. Then, the control law $\left(\nu=-\lambda \widehat{L_{s}^{+}} e\right)$, with $e=s-s^{*}$ and $\lambda$ a positive gain, becomes:

$$
\nu_{x y}=-L_{x y}^{+}\left(\lambda e+L_{z} \nu_{z}\right)
$$

where $\nu_{z}$ can be computed by calculating separately rotation and translation obtaining linear and angular velocity commands that can be sent separately and simultaneously to the multirotor in order to achieve translation and rotation. In this way, rotation can be controlled by $\omega_{z}=\lambda_{\omega_{z}}\left(\psi_{t}^{*}-\psi_{t}\right)$ where $\psi_{t}$ and $\psi_{t}^{*}$ are the orientation of the target in the image and its desired value, respectively. Altitude can be controlled by $v_{z}=\lambda_{v_{z}} \ln \left(\sigma^{*} / \sigma\right)$ where $\sigma$ and $\sigma^{*}$ are the area and its desired value of the polygon enclosed by the set of features $s$. In this work, we use the partitioned IBVS approach as another baseline technique in order to evaluate the proposed RL-IBVS controller.

All the baseline techniques, that is, classic, classic adaptive, and partitioned IBVS, have been implemented using the Visual Servoing Platform (ViSP) library (Marchand et al., 2005).

\subsubsection{IBVS through Reinforcement Learning controller}

The proposed RL-IBVS is based on our own designed reinforcement learning framework (see Fig. 4.1), which integrates an agent (i.e., an aerial robot executing an RL algorithm) which can interact with an environment based on RotorS Gazebo simulator.

The design of our reinforcement learning framework focuses on the two core levels of abstraction in reinforcement learning problems: the agent and the environment, providing a flexible and versatile interface for the development of new agents or environments. In the proposed RL framework, the interaction between the agent and the environment has been implemented following the guidelines provided by the OpenAI Gym (Brockman et al., 2016) toolkit for reinforcement learning, using ROS for communication purposes.

In this section, we model the visual servoing task as a fully observable Markov Decision Process (MDP), where the final objective is to train the agent in order to find the policy $\pi$ that maximizes the accumulated discounted reward $R_{t}=\sum_{i=t}^{T} \gamma^{(i-t)} r\left(\boldsymbol{s}_{i}, \boldsymbol{a}_{i}\right)$, given the state $\boldsymbol{s}_{i}$, the action $\boldsymbol{a}_{i}$, and a discount factor $\gamma \in[0,1]$. The behavior of the agent is controlled by this policy which maps an observation to an action, and is usually evaluated by using the action-value function $Q^{\pi}\left(\boldsymbol{s}_{t}, \boldsymbol{a}_{t}\right)=\mathbb{E}_{\pi}\left[R_{t} \mid \boldsymbol{s}_{t}, \boldsymbol{a}_{t}\right]$, which computes the expected return taking action $\boldsymbol{a}_{t}$ at state $\boldsymbol{s}_{t}$ and then following policy $\pi$ thereafter. 


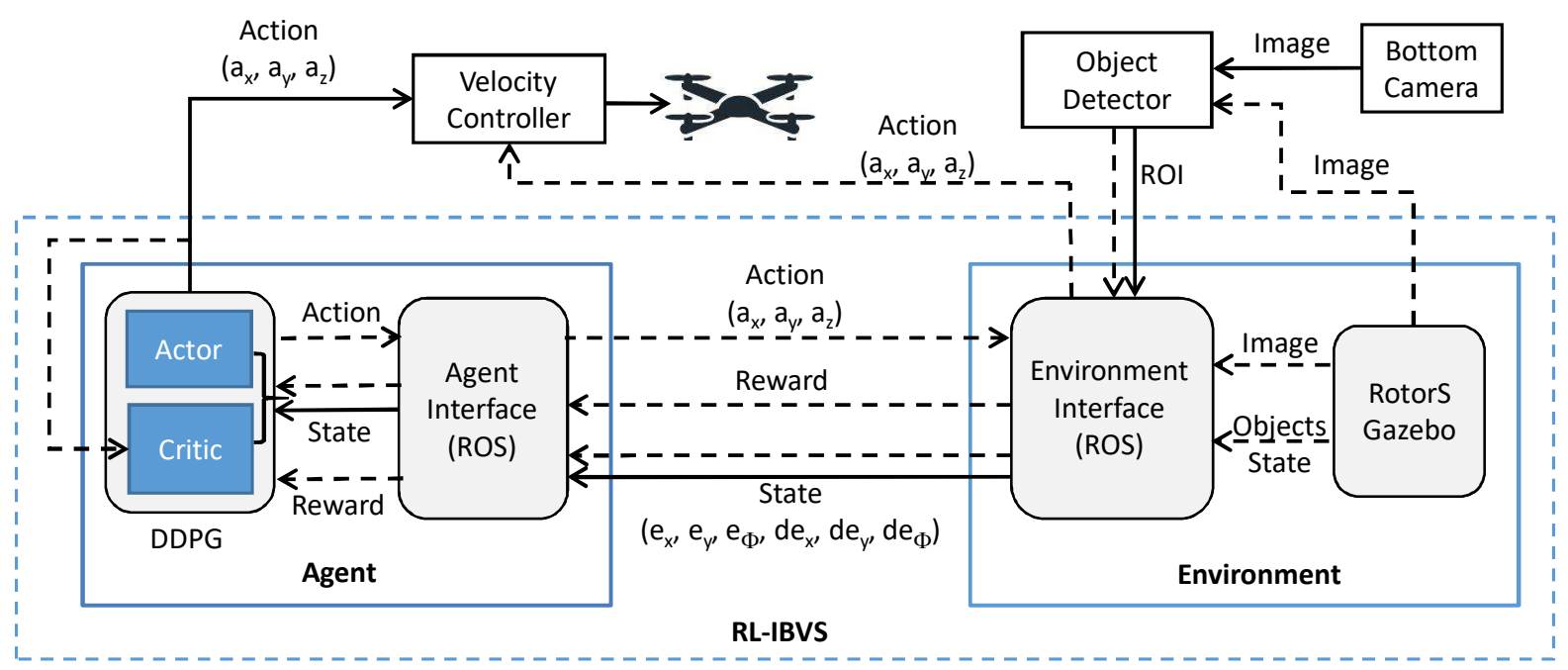

Figure 4.1: Architecture of the proposed RL-IBVS system. The enviroment receives as input an ROI in the image plane computed by an object detector. The agent, which implements the DDPG algorithm, receives an observation and a reward from the environment and computes the action (linear velocity) to be commanded to the velocity controller. The dotted lines represent interactions between the components in training mode, while the continuous lines depict the interactions in test mode.

In the proposed RL-IBVS architecture, the agent implements the DDPG algorithm, which is a model-free, off-policy algorithm based on an actor-critic architecture. In this architecture, the critic neural network acts as a nonlinear function approximator for estimating the action-value function, while the actor neural network is in charge of learning the policy that maps a state into a continuous action. We refer the reader to Section A.2 in Appendix $\mathrm{A}$ for a detailed explanation of the DDPG algorithm. At each time step $t$ the agent executes a continuous action $\boldsymbol{a}_{t}$ and receives an observation (next state $\boldsymbol{s}_{t+1}$ ) and a reward from the environment (see Fig. 4.1).

In the next sections, we describe the RL-IBVS variants that have been implemented for controlling the UAV in $2 \mathrm{D}$ and $3 \mathrm{D}$ control modes.

\section{D RL-IBVS controller}

This controller has been presented in Sampedro et al., 2018b for the interaction of the UAV with a moving target in search and rescue (SAR) missions.

In the specific case of the 2D RL-IBVS, the observation computed by the environment consists of a 4 -dimensional vector $\boldsymbol{s}_{t}=\left(e_{x}, e_{y}, d e_{x}, d e_{y}\right)$, where $e_{x}, e_{y}$ represent the normalized error in position, measured in pixels, of the center of the detected target ROI with respect to the defined reference in the image (see Fig. 4.2), and $d e_{x}, d e_{y}$ are the normalized derivatives of the error filtered using a low-pass filter. The normalization factor utilized includes the width and the height of the image for the $x$ and $y$ coordinates respectively. On the other hand, the action generated by the agent consists of a two dimensional vector $\boldsymbol{a}_{t}=\left(a_{x}, a_{y}\right)$, where $a_{x}, a_{y}$ represent the linear velocities in the $x$ and $y$ direction commanded to the Motion Controller (Pestana et al., 2014) in global coordinates, generated 
by the deterministic policy $\pi\left(s_{t}\right)$ computed by the actor network.

Regarding the neural networks that compose the DDPG, both the actor and critic networks consist of fully-connected neural networks with two hidden layers of 300 and 200 units, featuring ReLU non-linearity. At test time, the only network utilized is the actor network, whose input consists of a 4-dimensional vector representing the state of the detected target in the image, with the output layer being composed of two units with a tanh activation function that provide the 2-dimensional action to be commanded to the Motion Controller of the UAV, in velocity control mode. The output layer of the critic network consists of one unit with a linear activation function in order to estimate the Q-value function.

The reward at each time step (see Eq. 4.6) is computed by considering the evolution of a shaping function (Dorigo and Colombetti, 1998) (see Eq. 4.5) in two consecutive time steps. This procedure permits to alleviate the temporal credit assignment problem while accelerating the learning process of the agent as a localized advice is provided to the agent in each transition via the shaping function (Laud, 2004; Erez and Smart, 2008). The latter has been carefully designed in this work for achieving a smooth desirable behavior of the UAV while approaching the target. For this purpose, as can be seen in Eq. 4.5, the behavior of the agent is penalized when the errors in the image, their velocities, or the commanded actions are high.

$$
\begin{gathered}
\operatorname{shaping}_{t}=-\alpha \sqrt{e_{x}^{2}+e_{y}^{2}}-\beta \sqrt{d e_{x}^{2}+d e_{y}^{2}}-\epsilon \sqrt{a_{x}^{2}+a_{y}^{2}} \\
r_{t}=\operatorname{shaping}_{t}-\text { shaping }_{t-1}
\end{gathered}
$$

where $r_{t}$ is the reward computed at time step $t$, and $\alpha, \beta, \epsilon$ are the gains that control the penalization in the error in position, velocity, and action terms respectively. These gains have been empirically obtained, being $\alpha=100, \beta=10$, and $\epsilon=1$.

\section{D RL-IBVS controller}

This controller has been presented in Sampedro et al., 2018c as an extension of the 2D RL-IBVS with the purpose of controlling the UAV in 3D scenarios for object-following missions.

In the specific case of the 3D RL-IBVS, the state computed by the environment at each time step consists of a 6 -dimensional vector defined by $\boldsymbol{s}_{t}=\left\{e_{x}, e_{y}, e_{\Phi}, d e_{x}, d e_{y}, d e_{\Phi}\right\}$, where $e_{x}, e_{y}$ represent the normalized error of the center of the detected ROI with respect to the reference defined in the image plane as stated for the 2D RL-IBVS, and $e_{\Phi}$ is the normalized error in diameter of the circumscribed circle $\left(\Phi_{o}\right)$ to the detected ROI with respect to the reference diameter $\left(\Phi_{r e f}\right)$ (see Fig. 4.2). The variables $d e_{x}, d e_{y}, d e_{\Phi}$ are the normalized derivatives of the error, filtered using a low-pass filter. On the other hand, the action generated by the actor network in the 3D RL-IBVS is defined by a 3-dimensional vector $\boldsymbol{a}_{t}=\left(a_{x}, a_{y}, a_{z}\right)$, where $a_{x}, a_{y}$, and $a_{z}$ are the linear velocities in the $x, y$, and $z$ coordinates, respectively.

The actor and critic neural networks that compose the DDPG agent of the 3D RLIBVS consist of fully-connected neural networks with two hidden layers of 400 and 300 
units, using ReLU activation function. The output layer of the actor network is composed of 3 units with a tanh activation function in order to generate the actions commanded to the velocity controller. The output layer of the critic network consists of one unit with a linear activation function in order to estimate the Q-value function.

The shaping function in the case of the 3D RL-IBVS is designed by mimicking the shaping function implemented in the 2D RL-IBVS presented in Eq. 4.5, in which we add analogous terms for considering the effect of the errors in diameter in order to infer altitude commands. Taking into account the above consideration, the behavior of the 3D RL-IBVS is modeled by the shaping function depicted in Eq. 4.7, and the reward function presented in Eq. 4.6.

$$
\begin{array}{r}
\operatorname{shaping}_{t}=-\alpha \sqrt{e_{x}^{2}+e_{y}^{2}}-\beta \sqrt{d e_{x}^{2}+d e_{y}^{2}} \\
-\delta\left|e_{\Phi}\right|-\epsilon\left|d e_{\Phi}\right|-\zeta \sqrt{a_{x}^{2}+a_{y}^{2}+a_{z}^{2}}
\end{array}
$$

where $\alpha, \beta, \delta, \epsilon$ are positive gains that control the penalization in the error in position and diameter in the image plane together with their derivatives. $\zeta$ represents the penalization in the action term. These gains have been empirically obtained, being $\alpha=100$, $\beta=10, \delta=100, \epsilon=10$, and $\zeta=1$.
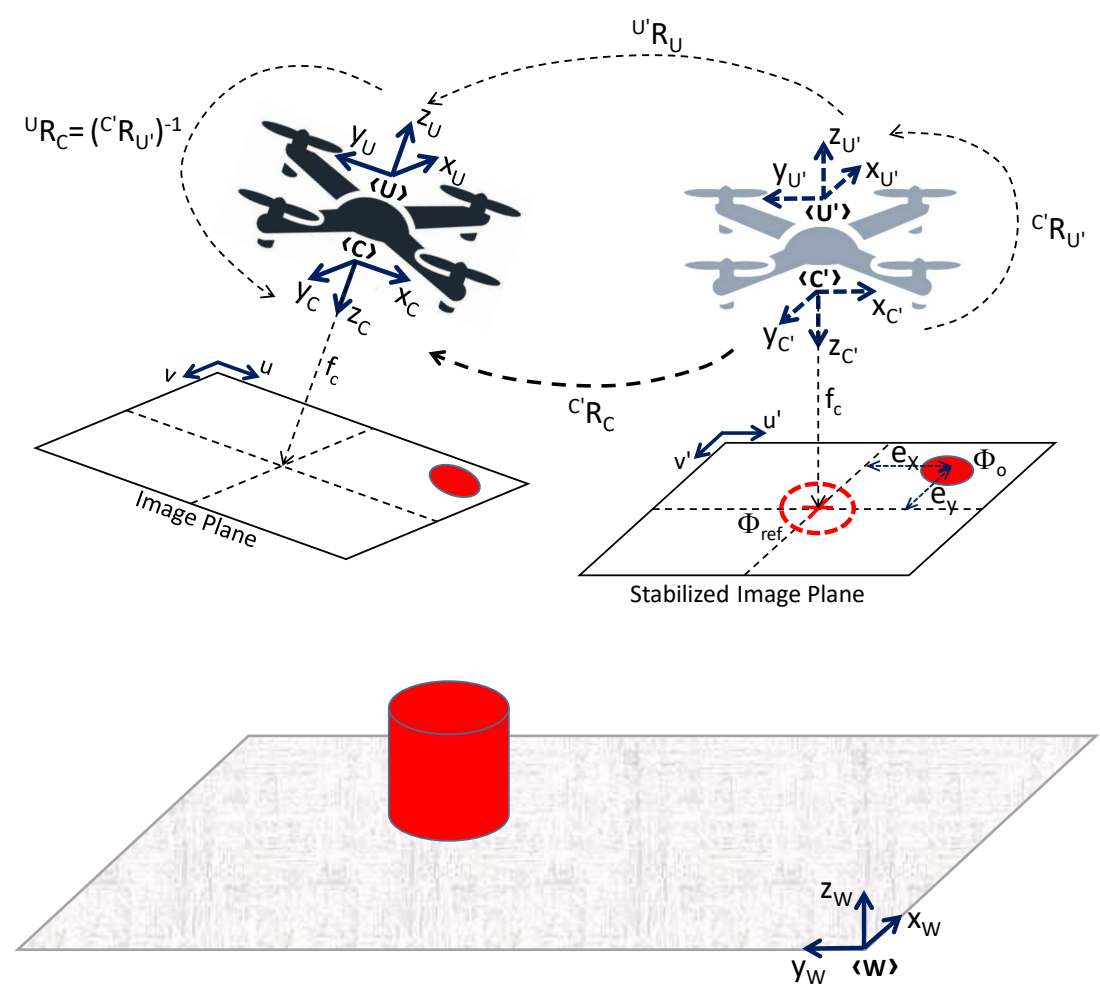

Figure 4.2: Notation and reference frames utilized in the RL-IBVS formulation. Where $W$, $C$ and $U$ stand for world, camera and aerial robot frames, respectively. The variables used for computing the state are also depicted in the stabilized image plane. 


\section{Image stabilization}

The errors in the image plane used to define the state, are computed with respect to a virtual stabilized image plane (see Fig. 4.2). In order to compute this virtual plane, we use the transformation in Eq. 4.8 derived from the pinhole camera model, and apply it to the current ROI taking into account the roll and pitch information provided by the Inertial Measurement Unit (IMU) of the aerial robot. This stabilization procedure has been found to be crucial in the learning process of the agent.

$$
\boldsymbol{p}^{\prime}=\underbrace{\boldsymbol{K} \cdot{ }^{C^{\prime}} \boldsymbol{R}_{U^{\prime}} \cdot{ }^{U^{\prime}} \boldsymbol{R}_{U} \cdot\left({ }^{C^{\prime}} \boldsymbol{R}_{U^{\prime}}\right)^{-1} \cdot \boldsymbol{K}^{-1}}_{\boldsymbol{H}} \boldsymbol{p}
$$

where:

$$
\begin{aligned}
& \boldsymbol{K}=\left[\begin{array}{ccc}
f_{x} & 0 & c_{x} \\
0 & f_{y} & c_{y} \\
0 & 0 & 1
\end{array}\right] \\
& C^{C^{\prime}} \boldsymbol{R}_{U^{\prime}}= \boldsymbol{R}_{z}(-\pi / 2) \boldsymbol{R}_{x}(\pi)=\left[\begin{array}{ccc}
\cos (\pi / 2) & -\sin (\pi / 2) & 0 \\
\sin (\pi / 2) & \cos (\pi / 2) & 0 \\
0 & 0 & 1
\end{array}\right]\left[\begin{array}{ccc}
1 & 0 & 0 \\
0 & \cos (\pi) & -\sin (\pi) \\
0 & \sin (\pi) & \cos (\pi)
\end{array}\right] \\
&{ }^{\prime} \boldsymbol{R}_{U}= \boldsymbol{R}_{z}(\psi) \boldsymbol{R}_{y}(\theta) \boldsymbol{R}_{x}(\phi) \\
&= {\left[\begin{array}{ccc}
\cos (\psi) & -\sin (\psi) & 0 \\
\sin (\psi) & \cos (\psi) & 0 \\
0 & 0 & 1
\end{array}\right]\left[\begin{array}{ccc}
\cos (\theta) & 0 & \sin (\theta) \\
0 & 1 & 0 \\
-\sin (\theta) & 0 & \cos (\theta)
\end{array}\right]\left[\begin{array}{ccc}
1 & 0 & 0 \\
0 & \cos (\phi) & -\sin (\phi) \\
0 & \sin (\phi) & \cos (\phi)
\end{array}\right] }
\end{aligned}
$$

In the above equations $\boldsymbol{p}$ represents a point in the current image plane, $\boldsymbol{p}^{\prime}$ is the point in the virtual stabilized image plane, both in homogeneous coordinates. $\boldsymbol{K}$ is the matrix containing the intrinsic camera parameters, that is, the focal length $\left(f_{x}, f_{y}\right)$ and the coordinates of the principal point $\left(c_{x}, c_{y}\right) .{ }^{C^{\prime}} \boldsymbol{R}_{U^{\prime}}$ is a $3 \times 3$ rotation matrix representing a fixed transformation from the camera (C) to the UAV frame of reference (U). ${ }^{U^{\prime}} \boldsymbol{R}_{U}$ is a $3 \times 3$ rotation matrix which defines the current orientation of the UAV (i.e., Euler angles yaw $(\psi)$, pitch $(\theta)$, and roll $(\phi))$.

Finally, the coordinates of any point in the current image plane can be expressed in the stabilized image plane using Eq. 4.9.

$$
\left[\begin{array}{l}
u^{\prime} \\
v^{\prime}
\end{array}\right]=\left[\begin{array}{l}
\frac{h_{11} u+h_{12} v+h_{13}}{h_{31} u+h_{32} v+h_{33}} \\
\frac{h_{21} u+h_{22} v+h_{23}}{h_{31} u+h_{32} v+h_{33}}
\end{array}\right]
$$

On the other hand, the reference in the image is computed by projecting the desired $3 \mathrm{D}$ points of the target object onto the image plane using the intrinsic camera parameters and considering a pinhole camera model. In this work, the desired 3D position of the robot with respect to the object is always considered centered with respect to the bottom camera frame $(\mathrm{C})$ and at the desired height above the object. 


\subsection{Experiments and Results}

In this section, we describe in detail the experiments that have been conducted for training and evaluating the proposed RL-IBVS algorithms. We first describe the experimentation that has been done for training successfully the proposed RL-IBVS controllers and then show the experiments performed to validate them. The 2D RL-IBVS has been integrated into a fully-autonomous system which will be further described in chapter 6 . A video demonstration showing the capabilities of the 2D RL-IBVS can be found in https://vimeo.com/235929544. Similarly, the results obtained for the 3D RL-IBVS can be reviewed in https://vimeo.com/258063350.

\subsubsection{Experimental setup}

In order to perform simulation and real flight experiments, the proposed 2D and 3D RL-IBVS algorithms have been integrated into the Aerostack framework (Sanchez-Lopez et al., 2016; Sanchez-Lopez et al., 2017a), which is built on top of the ROS middleware (Quigley et al., 2009), and provides the necessary additional software components (e.g., state estimator, velocity controller, etc.) in order to conduct autonomous flights. As mentioned previously, all the baseline IBVS methods, have been implemented using the Visual Servoing Platform (ViSP) library ${ }^{1}$

\section{Simulation experiments}

Different simulation scenarios have been created using the RotorS Gazebo simulator (see Fig. 4.3) in order to train and evaluate the proposed RL-IBVS variants, in which an AscTec Hummingbird quadrotor has to perform visual servoing maneuvers over a cylindrical object utilized as the target. The Hummingbird quadrotor is equipped with a fisheye-lens bottom camera with a horizontal field of view of $132^{\circ}$ and an image size of $640 \times 480$ pixels. For training the RL-IBVS agent, TensorFlow $2^{2}$ library on an Nvidia GeForce GTX 970 GPU is utilized under Ubuntu 16.04. Two Gazebo simulation scenarios have been created in order to test the RL-IBVS variants and compare them to the IBVS approaches presented in Section 4.3.1. For testing the capabilities of the 2D RL-IBVS we use a moving bucket which performs a rhomboidal trajectory in 2D. On the other hand, the 3D RL-IBVS is evaluated in a 3D object following configuration, using an AscTec Firefly as the leader which performs different $3 \mathrm{D}$ trajectories.

\section{Real flight experiments}

Several real flight experiments have also been conducted in order to test the capabilities of the different IBVS approaches on a real aerial robotic platform. For this purpose, two different aerial robotic platforms have been used. The 2D RL-IBVS has been tested onboard a custom designed UAV (see Fig. 4.4b), which is integrated by an Intel NUC6i5SYK featuring a $2.9 \mathrm{GHz}$ Intel Core i5-6260U CPU, and a standard RGB $180^{\circ}$

\footnotetext{
${ }^{1}$ https://visp.inria.fr/

${ }^{2}$ https://www.tensorflow.org/
} 


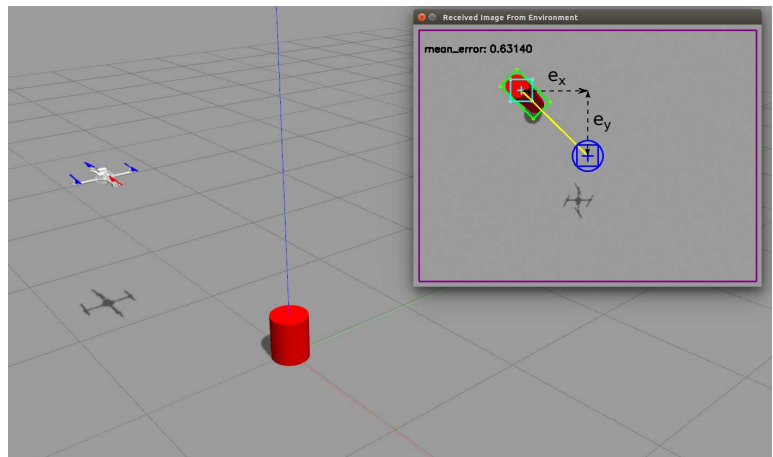

(a)

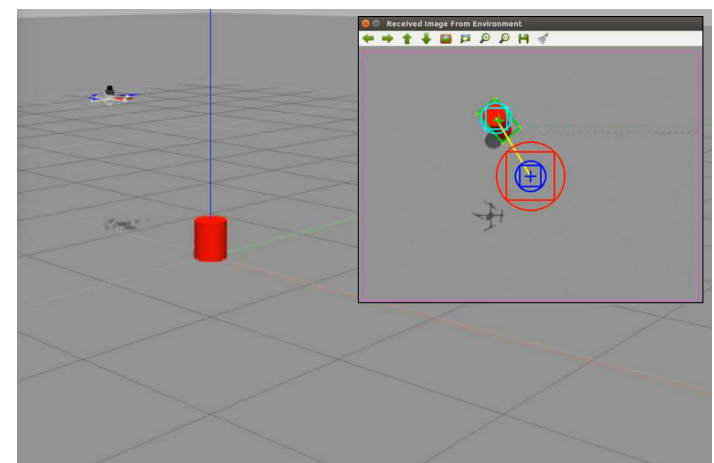

(b)

Figure 4.3: RotorS Gazebo simulation scenarios used for training the 2D (a) and 3D (b) RLIBVS controllers, and image captured from the bottom camera of the UAV (best seen in color). The desired reference is depicted in blue color, while the detection of the target at the current time step is shown in green color. The stabilized detection, considering the four corners of the square circumscribed to the circumference that forms the cylinder cover, is shown in cyan color. (a) Gazebo scenario used for training the proposed 2D RL-IBVS. (b) Gazebo scenario used for training the proposed 3D RL-IBVS. In this case, we also depict in red color the maximum diameter obtained at the lowest limit in altitude.

fisheye-lens bottom-looking camera with an image size of $640 \times 480$ pixels. The avionics of the UAV are managed by a Pixhawk autopilot.

Regarding the real flight experiments conducted to validate the proposed 3D RL-IBVS, we use a Parrot Bebop 2 which is equipped with a Parrot S.L.A.M. dunk (see Fig. 4.4a), utilized in this work only for obtaining velocities estimation, and a camera with an image size of $856 \times 480$ pixels. All the images captured onboard are sent via WiFi connection to the ground computer, featuring an Intel Core i7-6700HQ CPU. An OptiTrack motion capture system is used in two real flight experiments only for gathering ground truth data relative to the state of the robot and the target.

In order to obtain a reliable comparison of the different IBVS approaches, in all the experiments we limit the commanded actions to the range $[-0.5,0.5] \mathrm{m} / \mathrm{s}$ and introduce them to the same velocity controller (Pestana et al., 2014).

\subsubsection{Training methodology}

In this section, we provide a detailed description of the methodology designed for training the proposed RL-IBVS controllers. First, we highlight the common training procedure used in both 2D and 3D RL-IBVS, and then we remark the implementation details of each algorithm.

In order to train the agent to perform IBVS tasks, we use the RL-IBVS component (see Section 4.3.2 in training mode to command an AscTec Hummingbird quadrotor in the Gazebo environments presented in Fig. 4.3. In the RL-IBVS training mode, the environment is designed in an episodic RL setting, where the agent's experience is divided into a series of episodes, each one composed of several training steps. In each training 


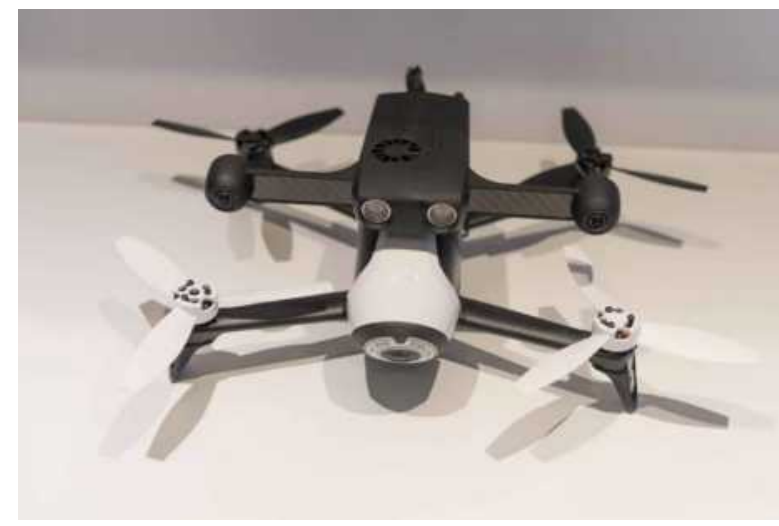

(a)

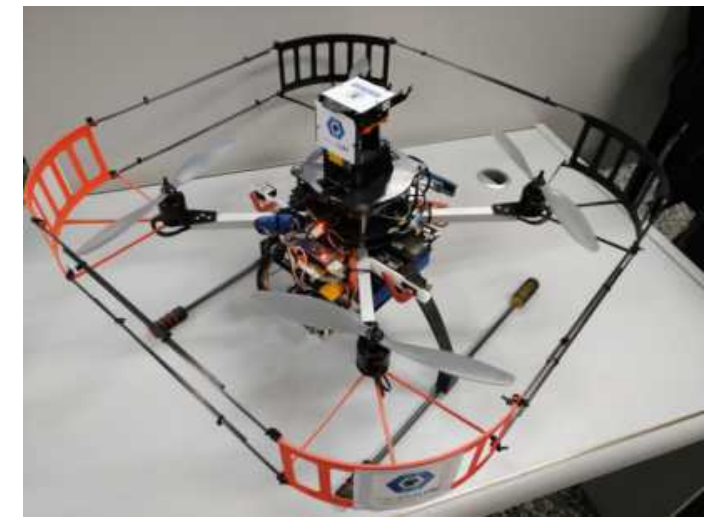

(b)

Figure 4.4: Aerial robotic platforms used for evaluating the IBVS algorithms in real flight scenarios. (a) Parrot Bebop 2 equipped with a Parrot S.L.A.M. dunk. (b) Custom-made aerial robot equipped with an Intel Core i5-6260U CPU and a fisheye-lens bottom-looking camera.

step, the agent, which runs at $20 \mathrm{~Hz}$, takes an action with added noise according to an Ornstein-Uhlenbeck distribution and receives an observation and a reward from the environment (see Fig. 4.1). The current observation is fed into the actor network, which generates a continuous action (linear velocity command) in the range $[-0.5,0.5] \mathrm{m} / \mathrm{s}$.

In order to stabilize and accelerate the training process, the variables from the state are measured taking into account the difference between the coordinates of the ROI obtained by the projection onto the stabilized image plane of the known $3 \mathrm{D}$ points of the target (see the cyan rectangle in Fig. $4.3 \mathrm{a}$ and $4.3 \mathrm{~b}$ ) with respect to the reference defined in the image plane (see the blue rectangle in Fig. $4.3 \mathrm{a}$ and $4.3 \mathrm{~b}$ ). This process is equivalent to the image stabilization technique explained in Section 4.3.2, which is used in test mode. During experimentation, we have found that this procedure is critical for allowing the convergence of the training process, as it removes large displacements of the detection of the target in the image plane due to sudden roll and pitch movements of the UAV.

During the training process of the actor and critic neural networks of both $2 \mathrm{D}$ and 3D RL-IBVS, Adam optimizer has been utilized with a base learning rate of $10^{-4}$ for the actor and $10^{-3}$ for the critic, and a minibatch size of 64 .

\section{D RL-IBVS}

At the beginning of each episode, the UAV is placed at a random position in a $2 \mathrm{~m}$ $\times 2 \mathrm{~m}$ area of the scenario at a constant altitude of $1.2 \mathrm{~m}$. The episode is considered finished when the agent reaches a terminal state. This situation can occur when the maximum number of steps per episode (400 steps) is reached or when the target is out of the boundaries defined in the image captured by the bottom camera of the UAV (see the magenta rectangle in Fig. 4.3a). When this situation occurs, the agent is penalized with a negative reward of -100 and a new episode is started. In the rest of situations, the reward is computed using Eq. 4.6, which depends on Eq. 4.5 .

Using the Adam optimization algorithm with the configuration described above and 
the actor and critic neural networks being composed of two hidden layers of 300 and 200 units, the training process of the actor and critic neural networks took approximately 7.5 hours. The results obtained during the training process are illustrated in Fig. 4.5. In this figure, it is important to notice the different time instants in which the reward and the action-value function stabilize. The former reaches a stable value around zero from episode 200, while the action-value function continues increasing until episode 1250. Based on these results, the learned weights adopted for the neural networks of the DDPG (actor and critic) are the ones obtained at episode 1400, whose results have been additionally confirmed in a visual manner, and can be reviewed in the video demonstration.

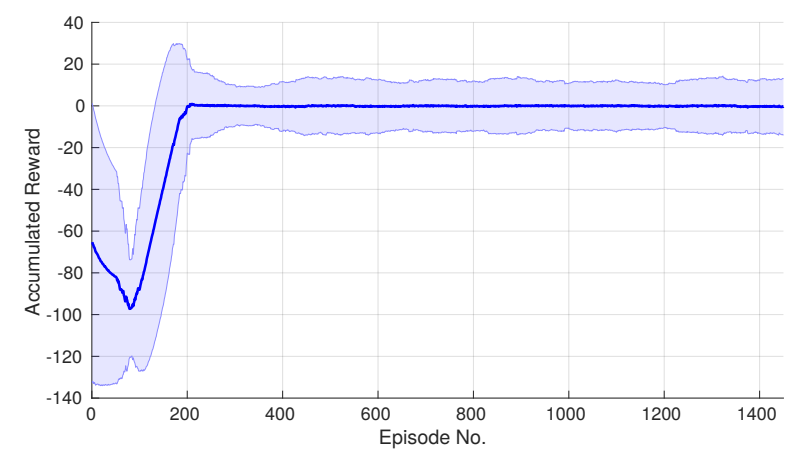

(a)

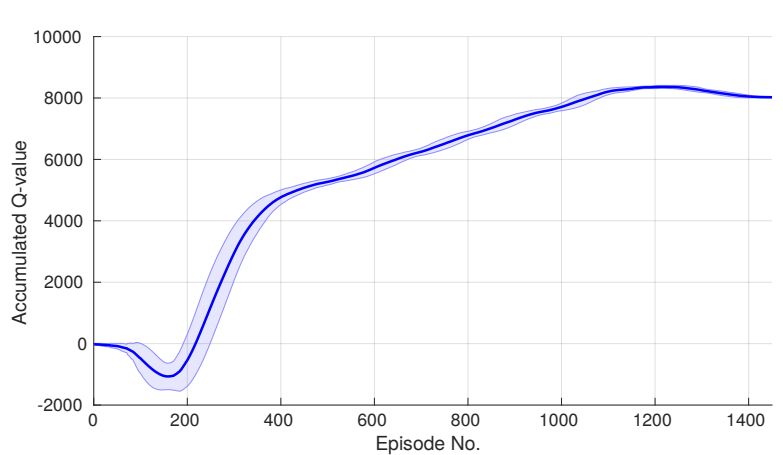

(b)

Figure 4.5: Training curves obtained during the training process of the 2D RL-IBVS agent. Each curve is computed taking into account a moving average of 100 episodes. (a) Each point depicts the accumulated reward at the specific episode. (b) Each point in the curve represents the accumulated action-value Q predicted by the critic network at the specific episode.

\section{D RL-IBVS}

In the training process of the 3D RL-IBVS, we increase the maximum number of steps per episode to 4000. In each episode, the agent is placed at a random position within an area of $2 \mathrm{~m} \times 2 \mathrm{~m} \times 1.7 \mathrm{~m}$ while the target object is static always at the origin. In each time step, the agent executes an action with added noise according to an OrnsteinUhlenbeck distribution with the final objective of being completely centered on the target object at the desired altitude with respect to it. A terminal state for finishing the current training episode of the 3D RL-IBVS can occur due to three main events: the target object is out of the image boundaries, the aerial robot is out of the altitude limits $(<0.7 \mathrm{~m}$ or $>1.7 \mathrm{~m}$ ), or the maximum number of steps per episode is reached. When any of the first two events occurs, the agent is rewarded negatively with -100. In the rest of the time steps, the agent is rewarded according to Eq. 4.6, which is computed using Eq. 4.7.

Using the Adam optimizer with the configuration described above and the actor and critic neural networks being composed of two hidden layers of 400 and 300 units, the training process of the 3D RL-IBVS agent took approximately 17 hours, learning a stable behavior around episode 1400, which can be reviewed in the training curves presented in Fig. 4.6. In this figure, we only show the behavior of the agent since episode 600 for clarity. 
From episode 0 to 850 , the agent is continuously giving very high positive and negative commands in altitude reaching a terminal state in few simulation steps. This fact produces the Q-value curve to have values around zero until episode 850. As shown in Fig. 4.6b the Q-value presents an extremely similar tendency as in the case of the 2D RL-IBVS, increasing until episode 1200 when the maximum value is reached, and stabilizing around episode 1350. The accumulated reward curve shown in Fig. 4.6a exhibits a more noisy response which is related to the terminal states that the UAV can reach. As compared to the training methodology of the 2D RL-IBVS, the training process of the 3D RLIBVS incorporates a terminal state related to the minimum and maximum altitude that the UAV can reach. These limits are easily reachable owing to the noise added to the commanded actions together with the small available area in height $(1 \mathrm{~m})$. Despite these hard constraints, the reward function shows an incremental tendency reaching the highest values after episode 1400. Based on the analysis of the accumulated reward and Q-value functions, together with a visual confirmation of the recorded experiments, the learned weights selected for the neural networks of the DDPG (actor and critic) are the ones obtained at episode 1400.

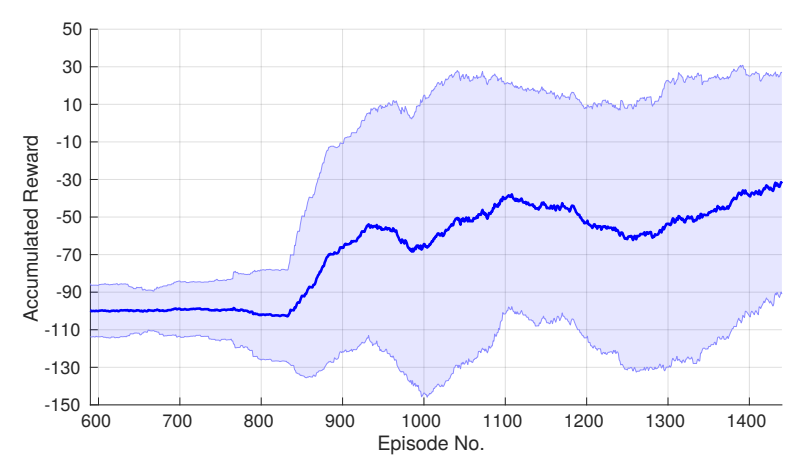

(a)

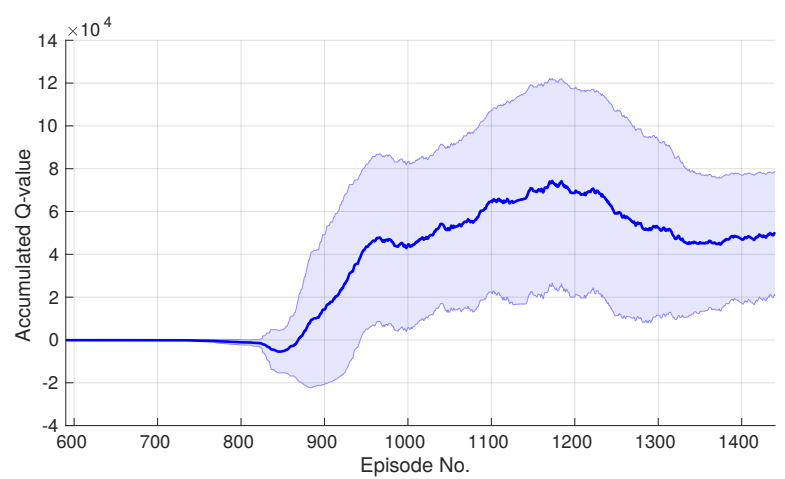

(b)

Figure 4.6: Training curves obtained during the training process of the 3D RL-IBVS agent. Each curve is computed taking into account a moving average of 100 episodes. (a) Each point depicts the accumulated reward at the specific episode. (b) Each point in the curve represents the accumulated action-value Q predicted by the critic network at the specific episode.

\subsubsection{Simulation Experiments}

This section presents the experiments that have been conducted under controlled simulation conditions using Gazebo in order to evaluate the proposed RL-IBVS variants and compared them to the IBVS baselines described in Section 4.3.1. The 2D RL-IBVS was the first algorithm developed and was evaluated using the classic IBVS described in Section 4.3.1. Afterwards, we enlarged the capabilities of the 2D RL-IBVS in order to build the 3D RL-IBVS, which has been evaluated using a wide range of baseline algorithms (i.e., the classic, classic adaptive, and partitioned IBVS). Since the 3D RL-IBVS integrates the 2D RL-IBVS, we consider that the evaluation of the 3D RL-IBVS is representative for 
analyzing the behavior of both RL-IBVS controllers.

\section{D RL-IBVS}

In this section, the experiments conducted in order to evaluate the proposed 2D RLIBVS algorithm using the trained agent are described in detail. For the sake of comparison, the IBVS controller proposed in Malis, 2004; Chaumette and Hutchinson, 2006a (referred to as classic IBVS) has been implemented using the ViSP library, and is utilized in this section as the baseline for evaluating the 2D RL-IBVS. The classic IBVS is based on the well-known visual servo control law which establishes that $\nu=-\lambda \widehat{L_{s}^{+}} e$, where $\lambda=0.75$ has been empirically found to perform appropriately.

In order to obtain a complete evaluation of the classic IBVS and the 2D RL-IBVS, a simulation experiment using RotorS Gazebo with an AscTec Hummingbird quadrotor has been designed with the aim of following a moving target. In this experiment, the target (i.e., a moving cylinder) performs a $2 \mathrm{D}$ rhomboidal trajectory (see Fig. $4.7 \mathrm{~d}$ and 4.8d), moving at a constant speed of $0.5 \mathrm{~m} / \mathrm{s}$ along the edges of the rhomboid and stopping during several simulation steps in its corners. Using this setup, a complete validation of the IBVS approaches can be conducted since it involves the interaction with a static and a moving target in the same experiment.

The results obtained after the execution of the proposed experiment are shown in Fig 4.7 and 4.8 for the classic IBVS and the RL-IBVS, respectively. As can be seen in Fig. 4.7a and 4.8a, both controllers generate a smooth response in the error signal, with a minimal oscillation in both the $x$ and $y$ directions. Regarding the actions generated by both IBVS controllers (see Fig. $4.7 \mathrm{c}$ and $4.8 \mathrm{c}$ ), it can be observed that the actions commanded by the classic IBVS have more noise than the ones commanded by the proposed 2D RL-IBVS. Despite this fact, the classic IBVS is able to maintain a stable trajectory while following the target with a very small deviation with respect to its trajectory (see Fig. $4.7 \mathrm{~d}$ ). The 2D trajectory generated by the 2D RL-IBVS is also stable (see Fig. 4.8d) with a slight deviation with respect to the trajectory of the target when it moves from point 2 to 3 . This can be caused owing to the higher error in the $x$ direction when the target executes the transition from point 2 to 3 .

Table 4.1 summarizes the results obtained for both IBVS approaches in the case of the error signal. The metrics presented in this table are the mean and standard deviation of the error signal, the steady-state error $(e(s s))$, and the percentage overshoot $(\mathrm{PO})$, which we consider relevant for the performance of an IBVS controller. The last two metrics (PO and $e(s s)$ ) are measured by averaging the response of the controllers to the 4 steps produced when the UAV approaches the moving target stopped at each of the four corners of the rhomboid (see steps 2-3-4-1 in Fig. 4.7a and 4.8a). In Table 4.1 it can be noticed the appropriate behavior of both controllers, highlighting the very high precision of the 2D RL-IBVS controller with an average error of less than one pixel and a steady-state error of less than 0.5 pixels in both $x$ and $y$ directions. Furthermore, the standard deviation of the error signal in the case of the RL-IBVS is about 6 pixels less than the classic IBVS, revealing the faster response of the former while following the target. The stable behavior of both controllers is evidenced when analyzing the PO. In this regard, the PO of both 


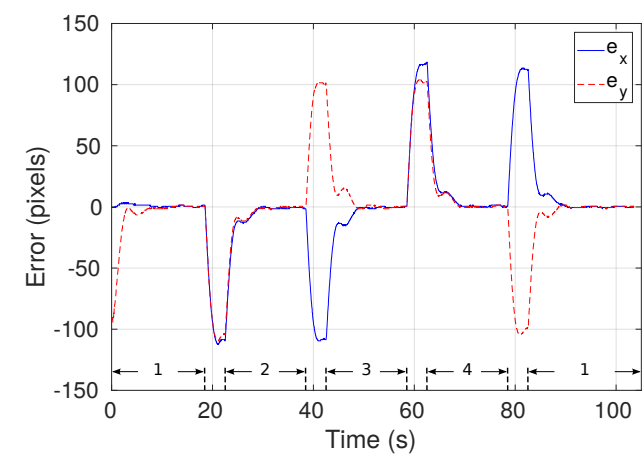

(a)

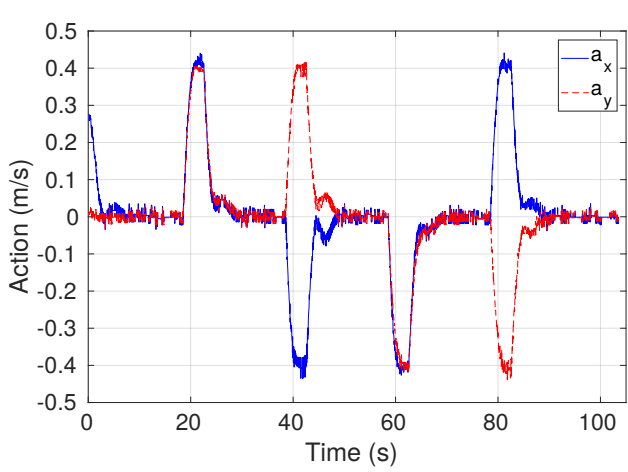

(c)

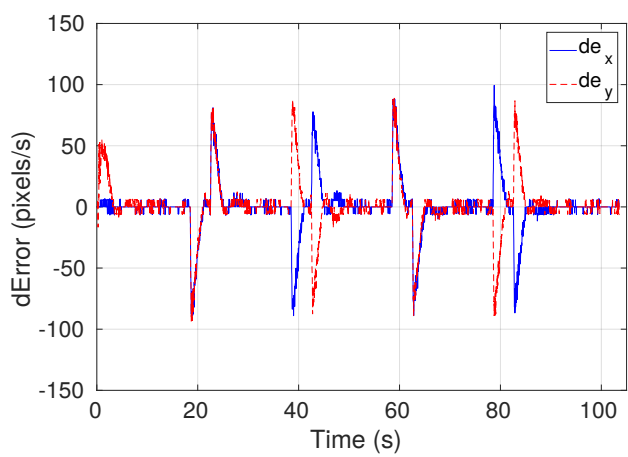

(b)

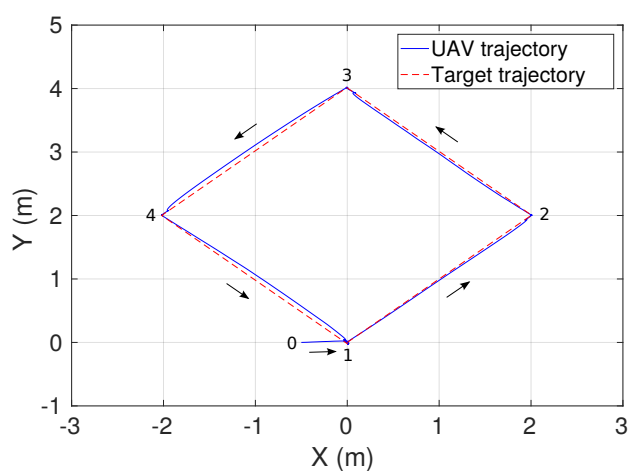

(d)

Figure 4.7: Simulation results obtained for the classic IBVS $(\lambda=0.75)$ approach while following a moving target executing a 2D rhomboidal trajectory at a maximum speed of $0.5 \mathrm{~m} / \mathrm{s}$. The target stops for a few time steps on each corner of the rhomboid (1 to 4). (a) Error in the image with respect to the reference. (b) Derivatives of the error in the image with respect to the reference. (c) Actions generated by the classic IBVS controller referred in the world frame of reference. (d) Trajectories followed by the UAV and the target.

controllers is on average less than $2.05 \%$ in both $x$ and $y$ directions.

It should be noted that the results obtained for the classic IBVS approach are the result of a long tuning process in which the corresponding gains of this controller were adjusted by a trial and error process. In contrast, the results presented in Fig. 4.8 for the 2D RL-IBVS have been obtained by directly using the trained actor network without any further tuning.

Table 4.1: Average errors measured in pixels and percentage overshoot obtained for the simulation flight experiment of Figures 4.7 and 4.8 .

\begin{tabular}{ccccccc}
\hline IBVS alg. & $e_{x}$ & $e_{y}$ & $e_{x}(s s)$ & $e_{y}(s s)$ & $P O\left(e_{x}\right)$ & $P O\left(e_{y}\right)$ \\
\hline Classic & $0.5 \pm 41.3$ & $-1.6 \pm 39.3$ & 0.28 & 0.74 & $0.67 \%$ & $1.49 \%$ \\
$2 D$ RL-IBVS & $-0.4 \pm 34.6$ & $-0.9 \pm 34.2$ & 0.25 & 0.11 & $2.03 \%$ & $0.68 \%$ \\
\hline
\end{tabular}




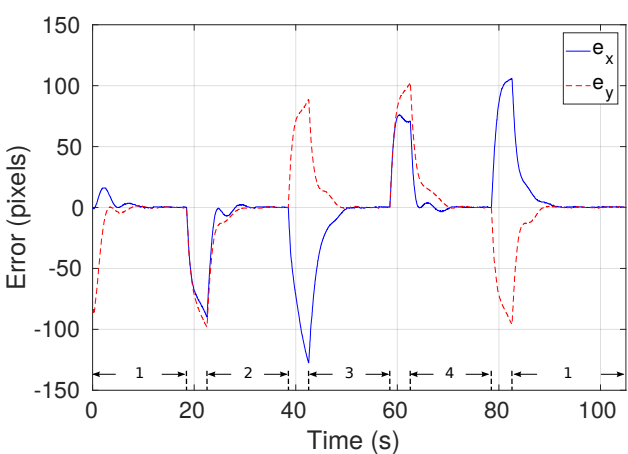

(a)

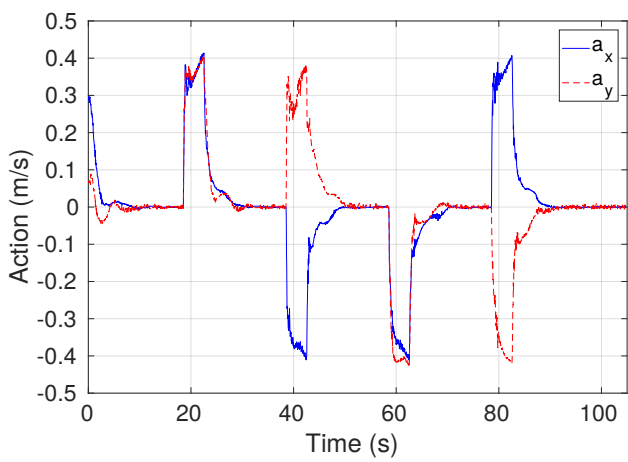

(c)

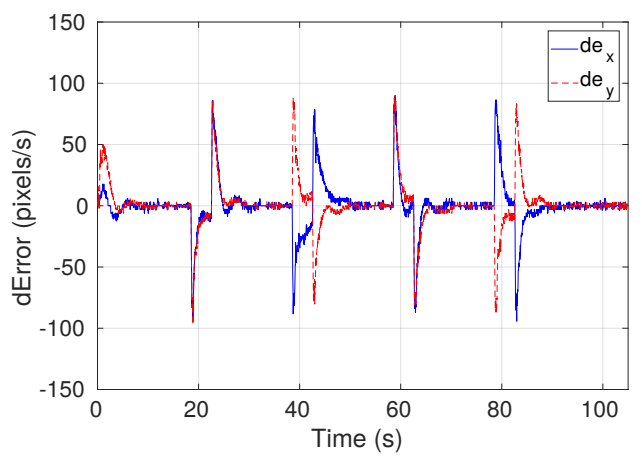

(b)

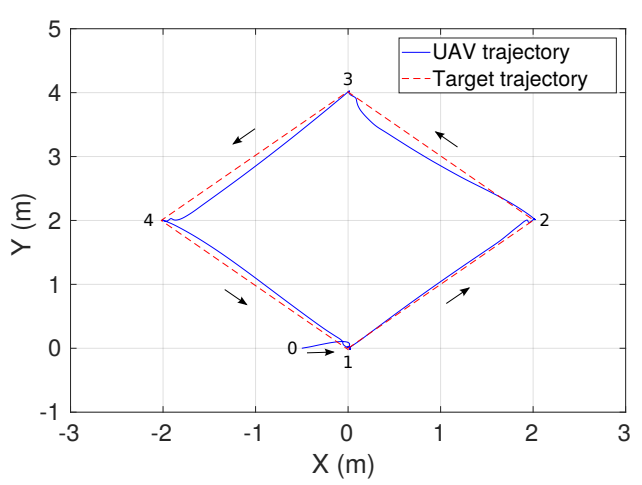

(d)

Figure 4.8: Simulation results obtained by the proposed 2D RL-IBVS controller while following a moving target executing a 2D rhomboidal trajectory at a maximum speed of $0.5 \mathrm{~m} / \mathrm{s}$. The target stops for a few time steps on each corner of the rhomboid (1 to 4). (a) Error in the image with respect to the reference. (b) Derivatives of the error in the image with respect to the reference. (c) Actions generated by the 2D RL-IBVS controller referred in the world frame of reference. (d) Trajectories followed by the UAV and the target.

\section{D RL-IBVS}

The aim of this section is to validate the proposed 3D RL-IBVS controller and compare it to the IBVS approaches presented in Section 4.3.1. For this purpose, a leader-follower experiment using two aerial robots has been designed using the RotorS Gazebo simulator (we refer the reader to the video demonstration for a better understanding). In the proposed experiment, an AscTec Firefly quadrotor is used as the leader and an AscTec Hummingbird acts as the follower. The leader performs a rhomboidal trajectory (top view) with an ascending or descending movement depending on the side of the rhomboid (see Figures 4.9c, 4.9f, 4.9i, and 4.91) at $v=0.33 \mathrm{~m} / \mathrm{s}$, and hovers during several simulation steps in its corners in order to evaluate the stabilization of each IBVS controller. The results obtained during the execution of the simulation experiment are shown in Fig. 4.9 and summarized in Table 4.2. As in the case of the 2D RL-IBVS, Table 4.2 shows the mean and standard deviation of the error signal, the steady-state error $(e(s s))$, and the percentage overshoot (PO). The last two metrics are obtained by averaging the responses 
to the 4 steps generated when the leader hovers at each of the corners of the rhomboid.

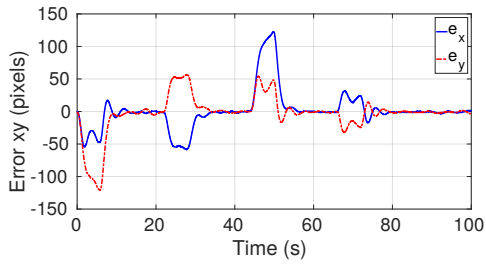

(a)

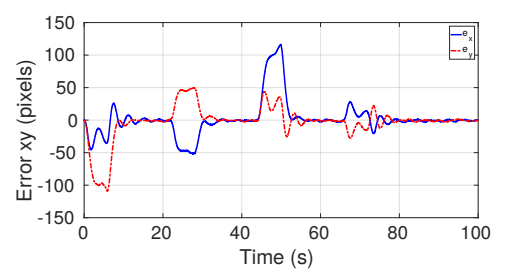

(d)

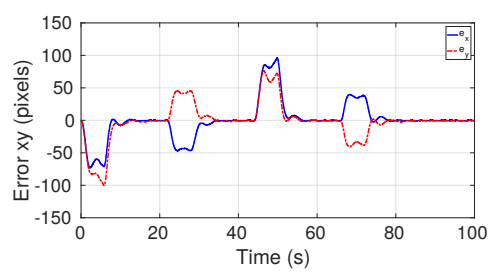

(g)

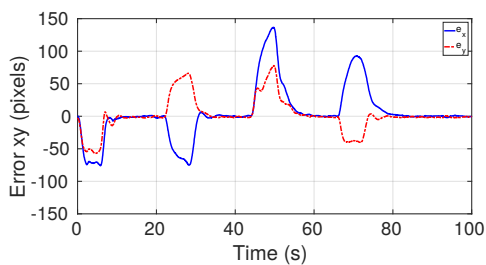

(j)

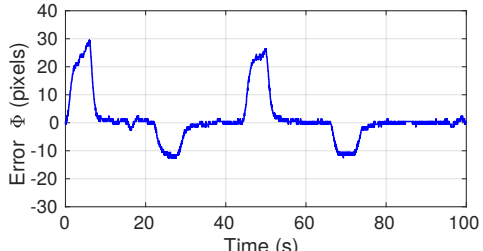

(b)

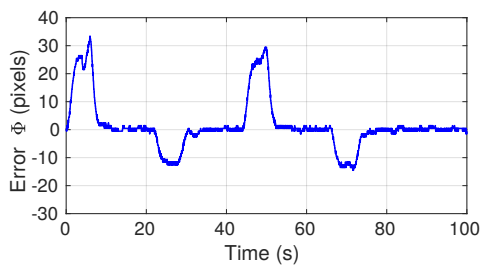

(e)

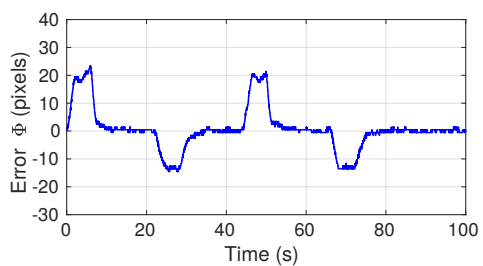

(h)

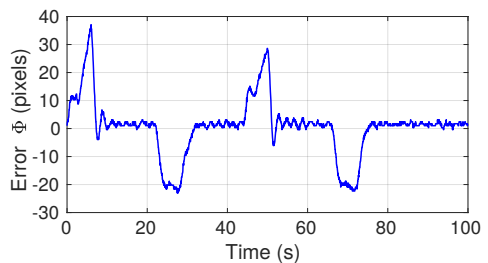

$(\mathrm{k})$

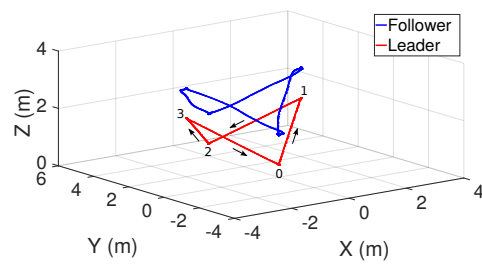

(c)

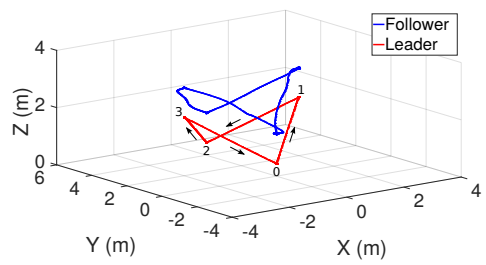

(f)

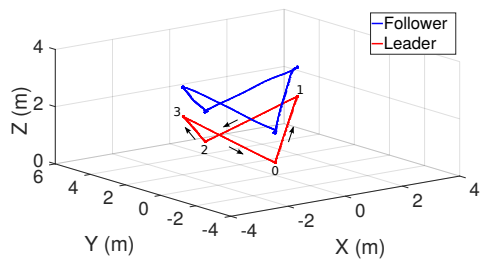

(i)

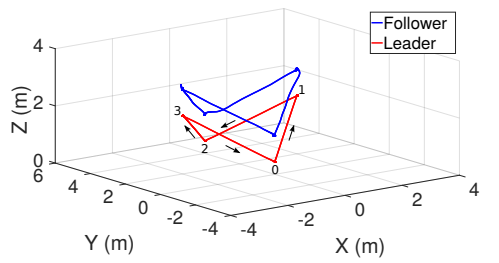

(1)

Figure 4.9: Simulation flight experiment for evaluating the proposed 3D RL-IBVS in a leaderfollower configuration, where the leader executes a rhomboidal trajectory (top view) hovering during some simulation steps at each corner. (a), (b), (c) Results obtained by the classic IBVS controller $(\lambda=1.05)$. (d), (e), (f) Results achieved using the classic adaptive IBVS $(a=1.67, b=$ $0.17, c=1.67)$. (g), (h), (i) Results achieved using the partitioned IBVS $\left(\lambda=\lambda_{v_{z}}=0.8\right) .(\mathrm{j})$, (k), (l) Results obtained with the proposed 3D RL-IBVS controller.

As can be seen in Fig. 4.9 and Table 4.2 , the partitioned IBVS is the controller which exhibits the most precise and smooth behavior, obtaining an average steady-state error of less than 0.5 pixels in all the error variables (i.e., $x, y$, and $\Phi$ ) and a PO less than $6.5 \%$ in all the cases. The proposed 3D RL-IBVS exhibits a similar accurate and smooth behavior in the $x$ and $y$ directions (see Fig. 4.9j) with average steady-state errors less than 1.5 pixels and PO less than $7.3 \%$. The response of the error signal in diameter is slightly more oscillatory than the baselines, what does not impede to obtain an accurate trajectory when following the target as shown in Fig. 4.91. The classic IBVS controllers 
Table 4.2: Average errors measured in pixels and percentage overshoot obtained for the simulation flight experiment of Fig. 4.9 .

\begin{tabular}{cccccccccc}
\hline IBVS alg. & $e_{x}$ & $e_{y}$ & $e_{\Phi}$ & $e_{x}(s s)$ & $e_{y}(s s)$ & $e_{\Phi}(s s)$ & $P O\left(e_{x}\right)$ & $P O\left(e_{y}\right)$ & $P O(\Phi)$ \\
\hline Classic & $0.7 \pm 29.1$ & $-2.5 \pm 29.2$ & $1.2 \pm 8.2$ & 0.91 & 1.54 & 0.47 & $28.0 \%$ & $25.31 \%$ & $11.14 \%$ \\
Classic_adap. & $1.6 \pm 27.1$ & $-2.7 \pm 26.4$ & $1.5 \pm 9.0$ & 0.77 & 0.73 & 0.48 & $56.51 \%$ & $56.90 \%$ & $8.05 \%$ \\
Partitioned & $0.2 \pm 28.0$ & $-1.6 \pm 28.4$ & $0.6 \pm 7.6$ & 0.42 & 0.44 & 0.47 & $3.39 \%$ & $4.33 \%$ & $6.40 \%$ \\
$3 D$ RL-IBVS & $5.4 \pm 40.2$ & $2.0 \pm 25.1$ & $0.7 \pm 9.8$ & 0.40 & 1.40 & 1.27 & $3.21 \%$ & $7.29 \%$ & $15.21 \%$ \\
\hline
\end{tabular}

are the ones that obtain the most oscillatory behavior, highlighting the response of the classic adaptive IBVS with PO of $56 \%$ in both $x$ and $y$ directions, which is related to the hard tuning process of this controller since it involves the tuning of 3 parameters (i.e., $a$, $b, c)$.

A final simulation experiment has been conducted in order to further validate in a qualitative manner the behavior of the IBVS controllers in an object-following mission where the target is commanded to perform a sinusoidal 3D trajectory (see Fig. 4.10c, 4.10f, 4.10i and 4.101). The results obtained after the execution of the experiment are shown in Fig. 4.10, which illustrates the values in each of the three error variables, and the trajectory generated by the target (i.e., the Firefly multirotor which acts as the leader) and the follower UAV commanded by the IBVS controllers. As can be observed in Fig. 4.10, all the IBVS controllers analyzed obtain a desirable behavior while following the target, highlighting the small error obtained in diameter for the partitioned IBVS and the proposed 3D RL-IBVS (see Fig. 4.10h and 4.10k), both less than 10 pixels during the whole mission. Regarding the response of the error signal in the $x$ and $y$ directions (see Fig. 4.10a, 4.10d, $4.10 \mathrm{~g}$, and $4.10 \mathrm{j}$ ), it can be observed that the classic and partitioned IBVS controllers are the ones which exhibit lower variances in the error signals. However, it should be noted that the gains of these controllers had to be readjusted to obtain a desired behavior. In contrast, the proposed 3D RL-IBVS exhibited a desirable behavior without the need to further modify the control actions generated by the actor network.

\subsubsection{Real-flight Experiments}

In this section, we describe the experiments that have been conducted to evaluate the proposed 2D and 3D RL-IBVS in different real-flight object-following missions (see Fig. 4.11). In Fig. 4.11a we show the experiment which simulates a search and rescue mission on a moving target in order to evaluate the 2D RL-IBVS. The scenario of this mission is limited to the $4 \mathrm{~m} \times 3 \mathrm{~m} \times 3 \mathrm{~m}$ volume corresponding to the OptiTrack motion capture system visible area. The same scenario is used in the object-following mission reproduced in the first experiment for evaluating the 3D RL-IBVS (see Fig. 4.11b), where the target is carried by a human operator which performs different movements according to a predefined 3D path. Finally, the capabilities of the proposed 3D RL-IBVS are further validated in a similar object-following mission using a remotely controlled car which executes an oval trajectory in a larger area of $8 \mathrm{~m} \times 8 \mathrm{~m}$ (see Fig. 4.11c). 


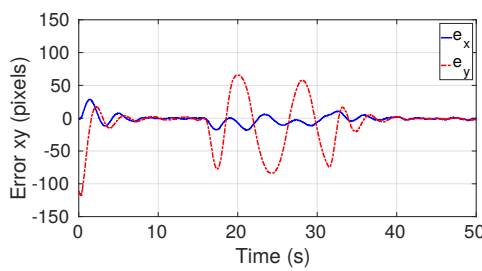

(a)

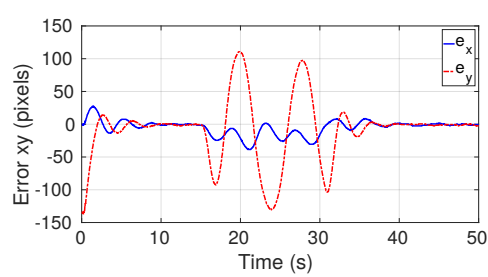

(d)

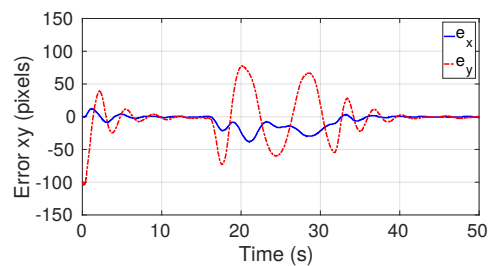

(g)

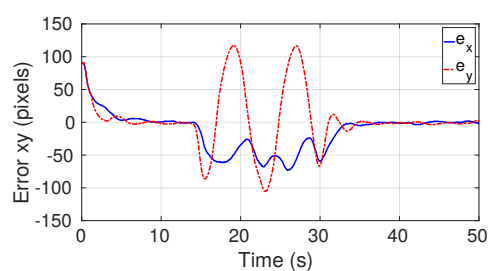

(j)

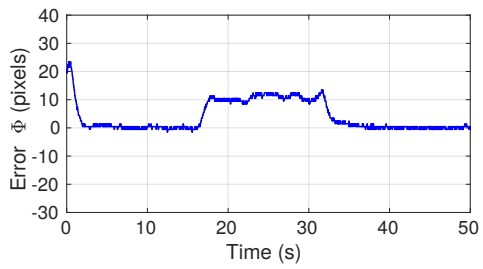

(b)

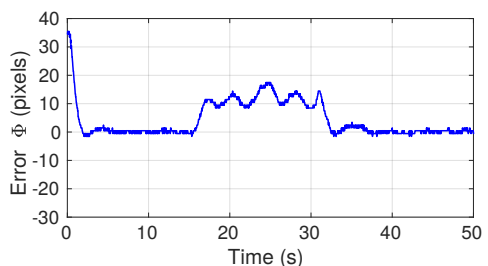

(e)

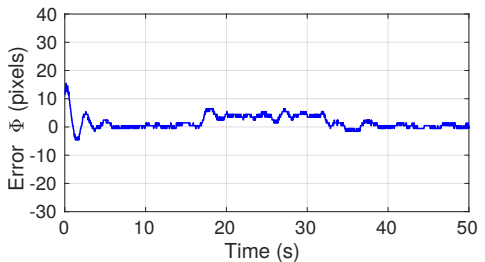

(h)

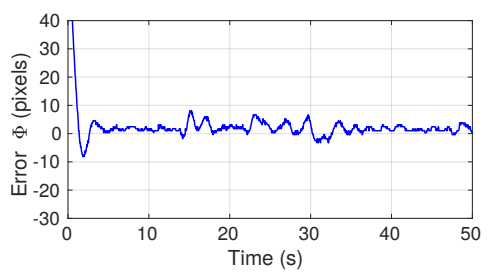

(k)

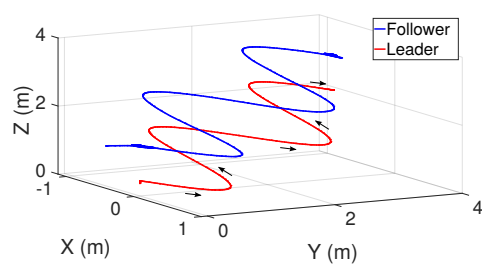

(c)

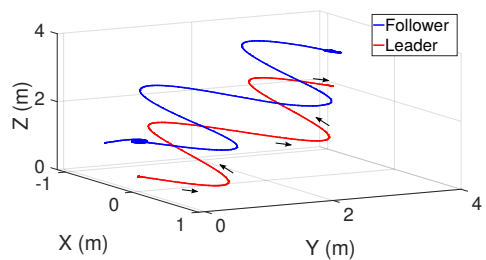

(f)

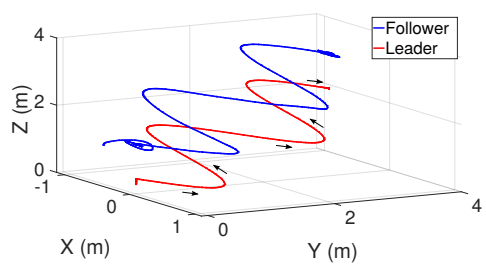

(i)

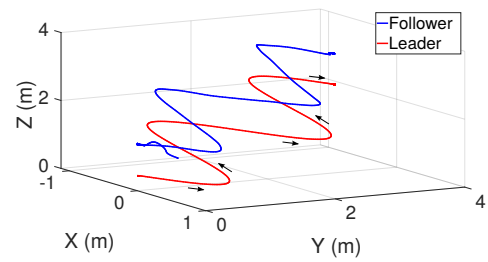

(l)

Figure 4.10: Simulation flight experiment for evaluating the proposed 3D RL-IBVS in a leaderfollower configuration, where the leader executes a sinusoidal trajectory. (a), (b), (c) Results obtained by the classic IBVS controller $(\lambda=1.8)$. (d), (e), (f) Results achieved using the classic adaptive IBVS $(a=1.67, b=0.17, c=1.67) .(\mathrm{g}),(\mathrm{h})$, (i) Results achieved using the partitioned IBVS $\left(\lambda=\lambda_{v_{z}}=1.8\right)$. (j), (k), (l) Results obtained with the proposed 3D RL-IBVS.

\section{D RL-IBVS}

The validation of the proposed 2D RL-IBVS in real flight conditions is motivated by its operation in search and rescue missions. A fully-autonomous system for the development of search and rescue missions will be presented in Chapter 6. In this section, we validate the proposed 2D RL-IBVS controller as the component responsible for the rescue operation of a moving target, which can execute random movements in any direction. To this aim, we integrate the 2D RL-IBVS controller into the aerial robot shown in Fig. 4.4b, which is additionally equipped with two small hooks to carry different objects (e.g., a medical kit) which need to be delivered to the target. Furthermore, in order to provide a realistic 


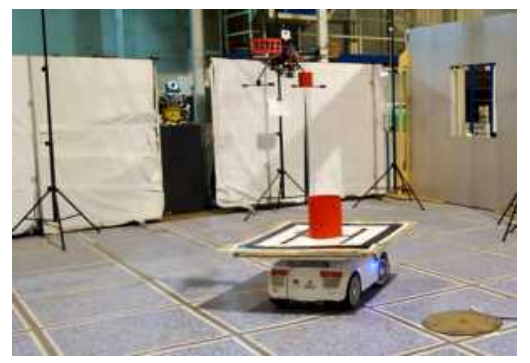

(a)

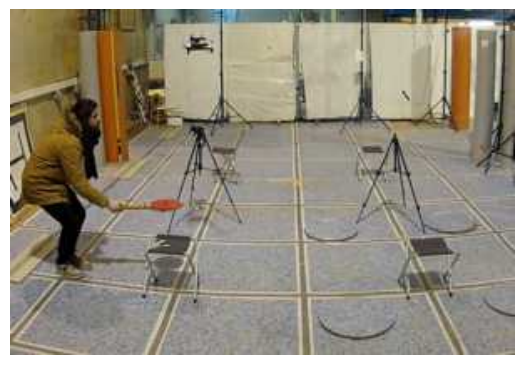

(b)

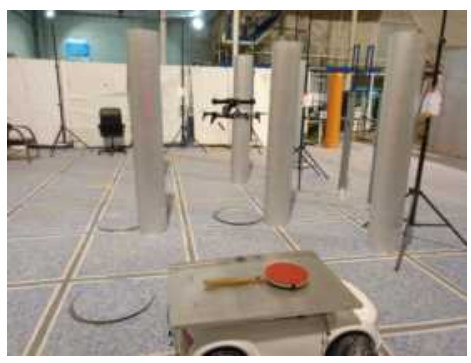

(c)

Figure 4.11: Scenarios created for real-flight experiments. (a) Scenario designed for evaluating the 2D RL-IBVS while performing a rescue operation on a moving target. (b), (c) Scenarios designed for evaluating the 3D RL-IBVS in two object-following missions.

SAR scenario, the 2D RL-IBVS is integrated into the Aerostack architecture, and more concretely, it communicates with the mission planner component (described in Chapter 6). Once the error in the image with respect to the target is below a predefined threshold, the 2D RL-IBVS is in charge of sending the appropriate signal of target locked to the mission planner, which will trigger the release command.

This search and rescue experiment integrates a remotely controlled moving target which describes a random trajectory commanded by a human operator. The goal of the UAV is to autonomously track the moving target until a secure position is reached for performing the release maneuver of a preloaded item for rescue purposes. The results obtained during the execution of the experiment are presented in Fig. 4.12, which shows how the error in the image and the control actions tend to zero at the end of the path of the moving target, where it starts decreasing the speed until it is stopped. In this time instant (see time instant $41.6 \mathrm{~s}$ in Fig. 4.12a), the error in pixels of the detected object in the image decreases below a predefined threshold and the target locked action for releasing the item is sent to the mission planner, which triggers its execution. In Fig. 4.12c , the decremental tendency of the control actions can be observed as soon as the UAV is approaching the target. Around time instant $41.6 \mathrm{~s}$, the release operation is commanded. The release of a $100 \mathrm{~g}$ item causes a perturbation on the dynamics of the UAV, successfully handled by the 2D RL-IBVS. Besides, it should be noted that despite the turbulences generated while flying very close to the target (empty bucket), the UAV is able to maintain a stable flight, being the control actions below $0.04 \mathrm{~m} / \mathrm{s}$ when the UAV is exactly above the target. Finally, the 3D trajectories followed by the UAV and the moving target are depicted in Fig. $4.12 \mathrm{~d}$, which shows the stable tracking trajectory followed by the UAV on top of the moving target. This trajectory is only perturbed in height when the release operation is executed. This fact can be observed around point $x: 0.84 \mathrm{~m}, y:-0.82 \mathrm{~m}$, when the UAV moves slightly up.

\section{D RL-IBVS}

Two main real flight experiments have been designed in order to evaluate the capabilities of the 3D RL-IBVS for commanding a real aerial robotic platform (see Fig. 4.11b 


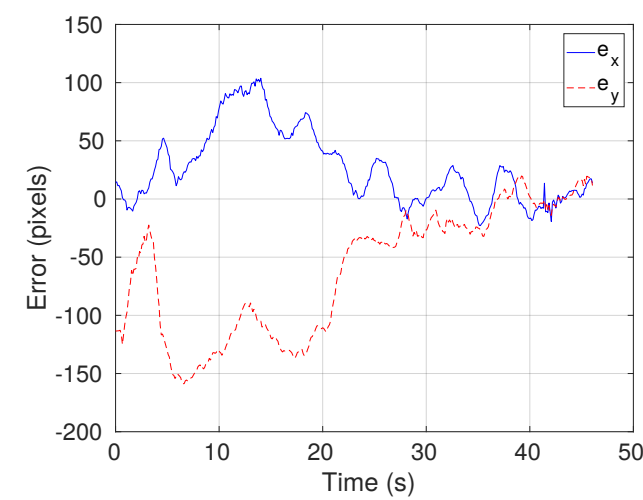

(a)

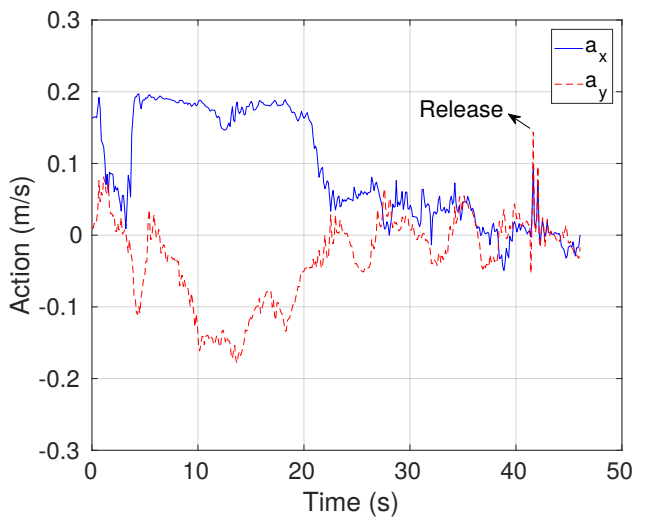

(c)

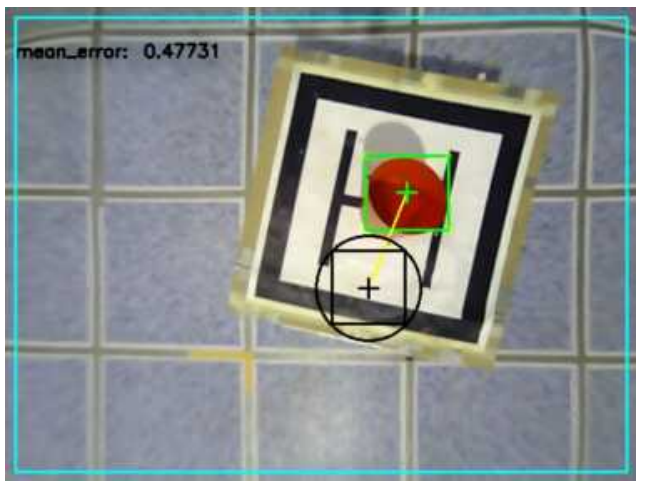

(e)

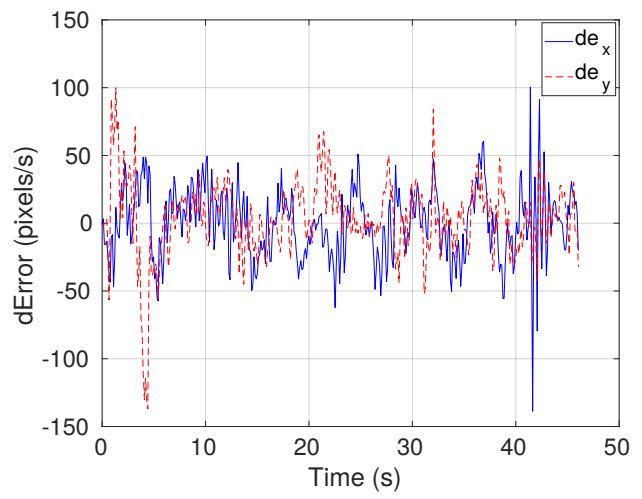

(b)

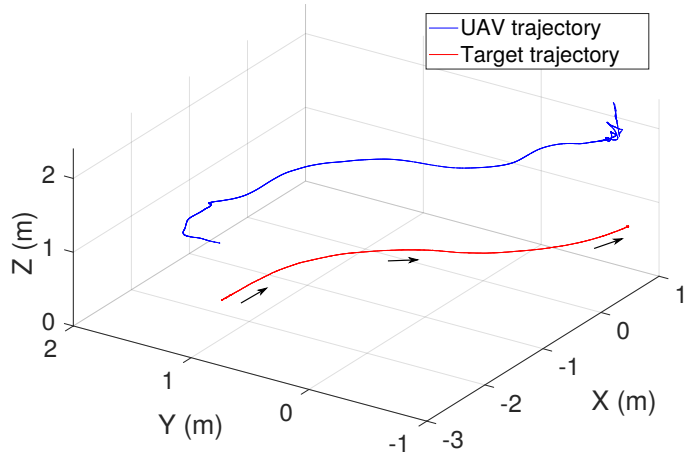

(d)

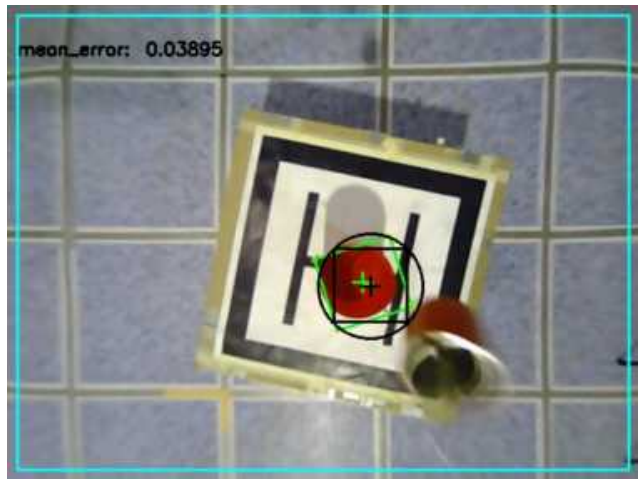

(f)

Figure 4.12: Real flight experiment for validating the proposed 2D RL-IBVS while performing a rescue mission on a moving target. (a) Error in the image w.r.t the reference. (b) Derivatives of the error in the image w.r.t the reference. (c) Action commands generated by the 2D RL-IBVS (in world frame of reference). (d) 3D paths followed by the UAV and the moving target. (e), (f) Errors computed by the 2D RL-IBVS component in two different instants of the mission. (f) When the target is locked, a release operation is commanded. An OptiTrack Mocap system is used for recording the positions of the UAV and the moving target. 
and 4.11c). In these experiments, we aim at reproducing the leader-follower configuration designed in the simulation experiments.

In the first experiment, the quadrotor leader has been substituted by a human operator carrying a target marker in order to obtain a smoother trajectory of the target for improving the comparative analysis. We have considered this configuration as the most suitable due to the high turbulences experienced when flying two small quadrotors one on top of the other. The human operator performs a continuous rectangular trajectory (top view) in a structured $4 \mathrm{~m} \times 3 \mathrm{~m}$ indoor scenario with an ascending and descending movement in each long side of a $3 \mathrm{~m} \times 2 \mathrm{~m}$ rectangle (see Fig. $4.13 \mathrm{c}, 4.13 \mathrm{f}$, and $4.13 \mathrm{i}$ ). An OptiTrack motion capture system is used to gather the information relative to the trajectories of the target and the UAV while performing the experiment. The results obtained after the execution of the experiment shown in Fig. 4.13 and summarized in Table 4.3 reveal the smooth behavior achieved by the proposed 3D RL-IBVS as compared to the baseline IBVS controllers. The proposed 3D RL-IBVS is able to maintain low errors in the $x$ and $y$ directions during the whole experiment as can be reviewed in Table 4.3 , where the variances in $x$ and $y$ are much smaller than those of the classic and partitioned IBVS. The signal related to the error in altitude (see Fig. 4.13h shows a more oscillatory response of the proposed 3D RL-IBVS, which can be caused by more aggressive movements of the target commanded by the human operator. Despite this fact, the 3D trajectory generated by the 3D RL-IBVS is very similar to the one generated by the target as shown in Fig. 4.13i, where it can be observed that the 3D RL-IBVS is able to maintain the altitude above the target during the whole mission.

Overall, the results presented in Fig. 4.13 demonstrate the appropriate capabilities of the proposed 3D RL-IBVS for being adapted to perform real flight experiments.

Table 4.3: Mean and standard deviation errors in pixels obtained for the real flight experiment of Fig. 4.13 .

\begin{tabular}{cccc}
\hline IBVS approach & $e_{x}$ & $e_{y}$ & $e_{\Phi}$ \\
\hline Classic & $19.9 \pm 94.7$ & $15.8 \pm 72.9$ & $-0.3 \pm 7.3$ \\
Partitioned & $-2.2 \pm 66.6$ & $12.8 \pm 58.1$ & $-1.8 \pm 5.7$ \\
$R L$ & $-10.9 \pm 52.5$ & $2.5 \pm 23.0$ & $-5.2 \pm 13.2$ \\
\hline
\end{tabular}

The second real flight experiment has been conceived as a visual servoing experiment using a remotely controlled car as the object to follow which performs an oval trajectory in an indoor scenario of $8 \mathrm{~m} \times 8 \mathrm{~m}$ (see Fig. 4.14). The aim of this experiment is to test the capabilities of the proposed 3D RL-IBVS while performing visual servoing in a larger area for following a moving car which executes sudden changes in its trajectory. The moving car is remotely controlled by a human operator who performs changes in the forward direction of the car when it moves along the $y$ axis (see Fig. 4.14a). Furthermore, several pillars are placed in the scenario in order to complicate the movement of the aerial robot. Despite the target executes sudden movements along its trajectory, the UAV commanded by the proposed 3D RL-IBVS is able to maintain a stable trajectory 


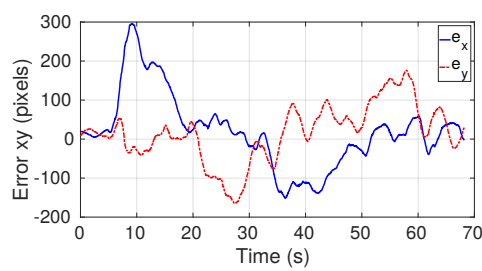

(a)

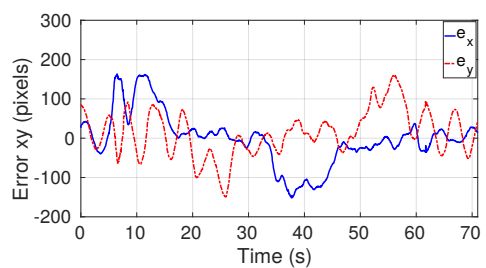

(d)

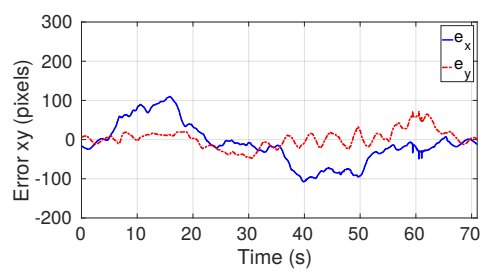

(g)

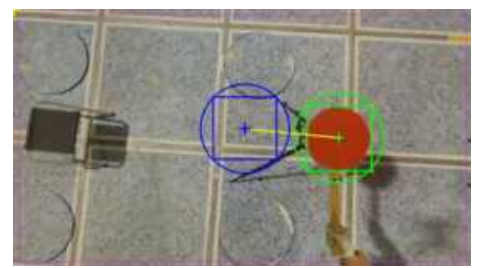

(j)

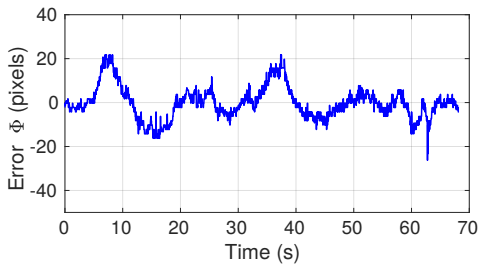

(b)

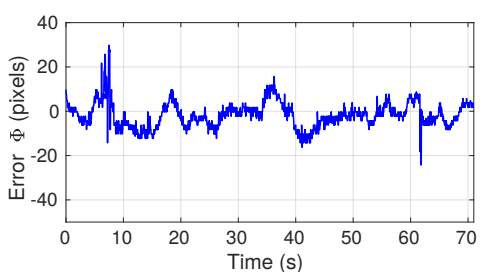

(e)

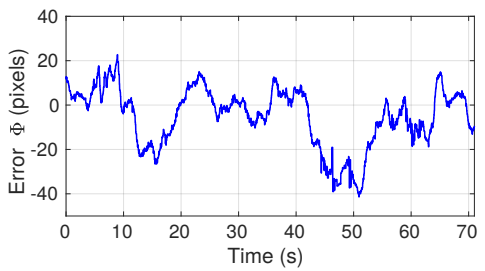

(h)

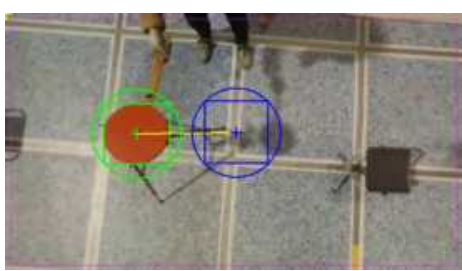

(k)

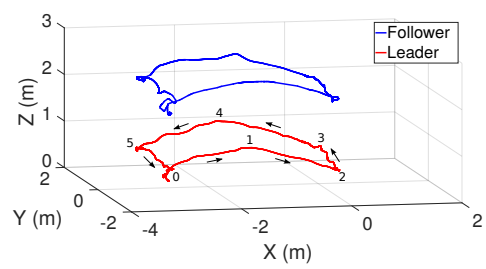

(c)

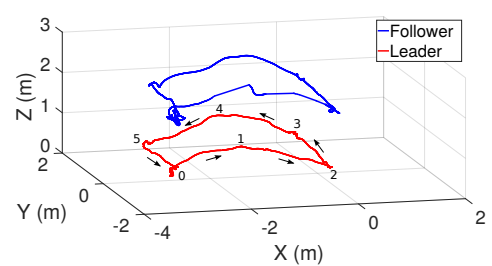

(f)

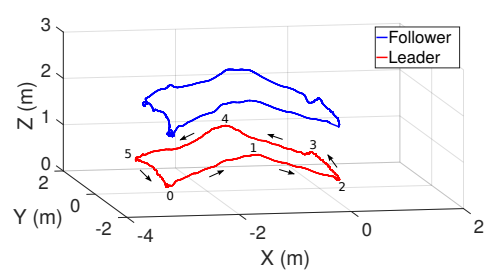

(i)

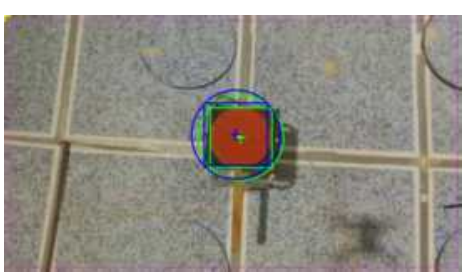

(1)

Figure 4.13: Real flight experiment for validating the proposed 3D RL-IBVS in a leader-follower configuration. The leader is characterized by a human carrying a target object. The follower consists of a Parrot Bebop 2 quadrotor. (a), (b), (c) Results obtained with the classic adaptive IBVS controller. (d), (e), (f) Results achieved using the partitioned IBVS. (g), (h), (i) Results obtained by the proposed 3D RL-IBVS controller. (j), (k), (l) Target detection and reference for the 3D RL-IBVS in different points of the trajectory (points 1, 4, and 0 or final point for images in $(\mathrm{j}),(\mathrm{k})$, and (l), respectively).

even in these critical points (see area around point A and B in Fig. 4.14a). Furthermore, as can be seen in Fig. 4.14b the metallic surface of the cover part of the car generates reflections which cause miss-detections of the target at certain instants during the objectfollowing mission. These situations do not seem to affect the performance of the proposed 3D RL-IBVS controller as soon as the detection is recovered without a big delay. 


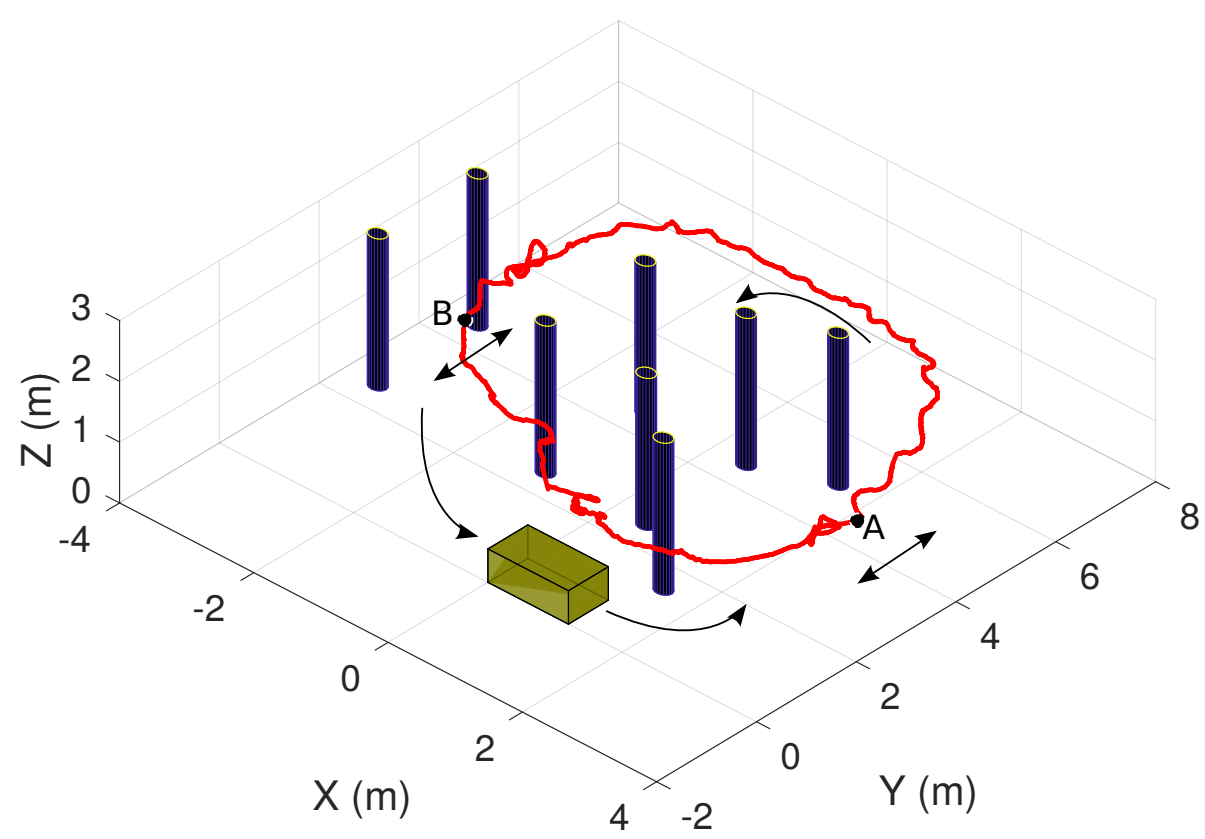

(a)

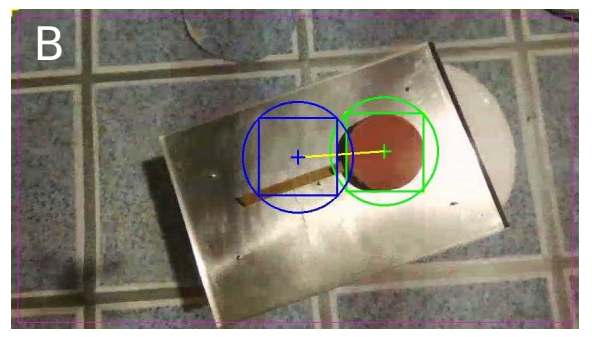

(b)

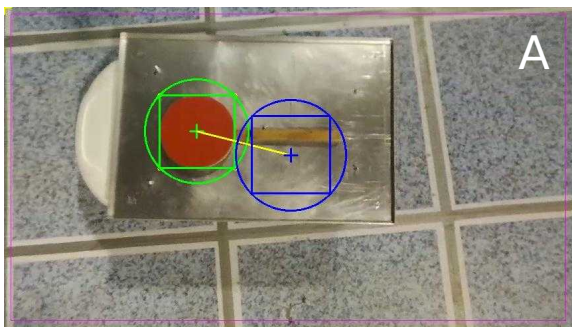

(c)

Figure 4.14: Real flight experiment for validating the proposed 3D RL-IBVS using a remotely controlled car as the object to follow. (a) Trajectory generated by the aerial robot (red color) while following the car (green cuboid). The arrows depict the movement of the car during the experiment. (b), (c) Detections of the target and visual servoing references in two instants (A and $\mathrm{B})$ of the trajectory.

\subsection{Discussion}

The results presented throughout this chapter for simulation and real-flight experiments demonstrate the appropriate capabilities of the proposed 2D and 3D Image-Based Visual Servoing Controllers through Reinforcement Learning (RL-IBVS).

Regarding the training process of the proposed RL-IBVS algorithms, the results presented in Fig. 4.5 and 4.6 reveal that the agent is able to learn appropriate IBVS policies, which is evidenced by the incremental tendency of the accumulated reward and the accumulated Q-value functions. The latter exhibit a very similar evolution in both $2 \mathrm{D}$ and $3 \mathrm{D}$ RL-IBVS (see Fig. 4.5b and 4.6b), where the accumulated Q-value predicted by the critic neural network shows and incremental tendency with two positive slopes until episode 1200 and then stabilizes around episode 1350. As can be seen in Fig. 4.6b, the accumu- 
lated Q-value corresponding to the 3D RL-IBVS reaches a stable value of $5 \times 10^{4}$, which is an order of magnitude higher than the stable Q-value achieved by the 2D RL-IBVS $\left(8 \times 10^{3}\right)$. This difference is due to the number of maximum steps per episode defined in each controller. The maximum number of steps defined in the case of the 2D RL-IBVS is inherited by other problems in RL where it is normal to select a few hundred steps per episode. Regarding the 3D RL-IBVS, in this case the number of maximum steps per episode was selected experimentally. We observed that incrementing this number allowed to learn more successful policies. However, having 4000 steps per episode greatly slows down the agent training process, which in the case of the 3D RL-IBVS took approximately 17 hours (on an Nvidia GeForce GTX 970 GPU) for learning a stable IBVS policy around episode 1350. The incremental tendency in the training process of the RL-IBVS agents is also noticed when analyzing the accumulated reward functions shown in Fig. 4.5a and 4.6a, The evolution of the accumulated reward function of the 2D RL-IBVS highlights that the agent is able to learn extremely fast, stabilizing its value around 0 after episode 200 . In contrast, the evolution of the reward function in the case of the 3D RL-IBVS reveals that incorporating an additional degree of freedom in altitude complicates the learning process. Furthermore, the terminal states designed for reseting the episode of the 3D RL-IBVS agent produce the high variance observed in the accumulated reward shown in Fig. 4.6a. Since the terminal state in altitude restricts the available displacement in height to $1 \mathrm{~m}$, the RL-IBVS agent can experience negative rewards of -100 in consecutive time steps when flying below $0.7 \mathrm{~m}$ or above $1.7 \mathrm{~m}$ (which is easy to reach due to the noise added to the commanded actions during training). Despite these restrictive conditions, the incremental tendency of the reward function of the 3D RL-IBVS continues even after episode 1400, which leads to thinking that its stabilization around 0 could occur later in the training process of the agent.

Overall, the results presented during the training process of both RL-IBVS agents validate the proposed strategy based on the difference between shaping functions in two consecutive time steps. These results also highlight the favorable properties of the shaping functions designed in this chapter and shown in Eq. 4.5 for the 2D RL-IBVS and Eq. 4.7 for the 3D RL-IBVS. Eq. 4.5 is based on an L2 energy function built around the errors in the $x$ and $y$ directions in the image. This is a well-defined function for minimization purposes which has shown to be effective for training the 2D RL-IBVS. The shaping function designed for training the 3D RL-IBVS incorporates two additional terms based on an L1 distance between the reference and detected diameter in the image. The combination of both energy functions works well as shown in Fig. 4.6, however, further analyses will be considered for studying alternative shaping functions in order to accelerate the convergence of the training process.

Once the RL-IBVS agents have been trained, the actor network of the 2D RL-IBVS consisting of a 4-layer fully-connected network composed of 4-300-200-2 neurons, and the actor network of the 3D RL-IBVS consisting of a 4-layer fully-connected network with 6400-300-3 neurons, are used for evaluating the RL-IBVS agents in test mode. To this aim, several simulation experiments have been designed in an object-following configuration. In the experiments presented in Fig. 4.7 and 4.8, the target consisting of a moving bucket executes a rhomboidal trajectory, stopping during several simulation steps in each of the 
corners of the rhomboid in order to measure the stabilization capabilities of the IBVS controllers. The results presented in Fig. 4.7 and 4.8 reveal the stable response of the classic IBVS and the 2D RL-IBVS controllers, highlighting the steady-state error below 0.5 pixels and PO below $2.05 \%$ obtained by the proposed 2D RL-IBVS. These results show the smooth behavior provided by the 2D RL-IBVS, which is further evidenced in Fig. 4.8c for the commanded actions (i.e., linear velocities in the $x$ and $y$ directions). As compared to the actions generated by the classic IBVS, with an average moving standard deviation of $8.3 \times 10^{-3}$ and $7.5 \times 10^{-3}$ considering the $x$ and $y$ directions (see Fig. $4.7 \mathrm{c}$ ), the proposed 2D RL-IBVS generates less noisy velocity commands with an average moving standard deviation of $2.3 \times 10^{-3}$ and $3.5 \times 10^{-3}$ considering the $x$ and $y$ directions, respectively. The latter metrics have been computed using a moving window of 5 samples for measuring the moving standard deviation in each of the step signals and averaging the results for the four steps.

Regarding the evaluation of the 3D RL-IBVS, a more complete set of simulation experiments involving object-following missions is presented in Fig. 4.9 and 4.10 for evaluating the performance of the proposed 3D RL-IBVS by comparing its behavior to several standard IBVS techniques. Similar to the performance of the 2D RL-IBVS, the results presented in Fig. 4.9 and summarized in Table 4.2 reveal the smooth and stable response of the 3D RL-IBVS. The proposed controller is able to achieve a steady-state error below 1.5 pixels in all the error variables considered (i.e., the errors in $x$ and $y$, and the error in diameter measured in the image plane) and a PO below $15.3 \%$ in all the variables, highlighting the PO of $3.2 \%$ in the $x$ axis. In this experiment, the most stable behavior is exhibited by the partitioned IBVS with steady-state errors less than 0.5 pixels and PO below $6.5 \%$ in all the error variables. However, it should be noted that these results are obtained after a time-consuming tuning procedure of the gains for the partitioned IBVS. In contrast, the results of the proposed 3D RL-IBVS are obtained by directly using the trained actor network, without any further modification. Only a small constant bias in the $x$ direction is removed from the error signal.

Further experiments for evaluating the performance of the proposed 3D RL-IBVS have been presented in Fig. 4.10 where the leader UAV is commanded to follow a 3D sinusoidal trajectory with a progressively increment in altitude. The results presented in Fig. 4.10 confirm the outstanding visual servoing capabilities of the proposed 3D RL-IBVS in object-following missions. In Fig. 4.10k, it can be observed that the 3D RL-IBVS is able to maintain a stable flight in altitude while following the target with errors in diameter less than 10 pixels during the whole mission. The partitioned IBVS behaves similarly in altitude with an error in diameter less than 10 pixels, and exhibits a more reactive behavior in the $x$ and $y$ directions with errors between $[-38,4]$ pixels in the $x$ axis, and between $[-74,75]$ pixels in the $y$ axis. Again, it should be noted that the gains of the classic and partitioned IBVS controllers were re-adjusted to obtain the best performance in the current experiment. In contrast, the proposed 3D RL-IBVS was not modified while switching between experiments what reveals the good capabilities learned by the 3D RL-IBVS, allowing its operation in a wide range of conditions.

Regarding the real flight experiments, different aerial robotic platforms and types of missions were considered for evaluating the performance of the proposed $2 \mathrm{D}$ and $3 \mathrm{D}$ RL- 
IBVS controllers. The usage of the 2D RL-IBVS was motivated for solving the rescue operations of moving targets in SAR missions. To this purpose, the 2D RL-IBVS was integrated into our custom-designed UAV. The validation of the 2D RL-IBVS in such conditions is shown in Fig. 4.12, where it can be observed the stable action commands generated by the proposed 2D RL-IBVS (see Fig. 4.12c), which are gradually decreasing as the UAV approaches the objective, and stabilizing between $[-0.04,0.04] \mathrm{m} / \mathrm{s}$ when the UAV is exactly above the target. These stable action commands allow obtaining a stable 3D path while following the target as shown in Fig. 4.12d. This path is only altered in height at the end of the mission where the UAV releases the $100 \mathrm{~g}$ item for completing the rescue operation, causing a perturbation in the dynamics of the UAV, which moves slightly up. Despite this perturbation (see instant 41.6s in Fig. 4.12a, 4.12b, 4.12c and 4.12d), which generates very high derivatives in the image error as shown in Fig. $4.12 \mathrm{~b}$, the proposed 2D RL-IBVS is able to efficiently handle this situation by commanding the corresponding actions (see instant 41.6s in Fig. 4.12c) in order to stabilize the UAV.

Similar object-following missions were designed for evaluating the proposed 3D RLIBVS. In this case, we used a commercial aerial robot platform (Parrot Bebop 2) equipped with a Parrot S.L.A.M. dunk for state estimation purposes. In this case, the proposed 3D RL-IBVS was running offboard and sending the control commands via WiFi to the aerial robot. Using this configuration, two real flight experiments were designed for following a human operator carrying a target consisting of a red marker, and a remotely controlled car carrying a red marker on top. The comparison of the 3D RL-IBVS with respect to classic and partitioned IBVS approaches shown in Fig. 4.13 provides important conclusions since the hard tuning process of both standard IBVS controllers can be an important constraint, which makes the proposed RL-IBVS an efficient alternative to the standard IBVS solutions. The behavior of the 3D RL-IBVS while following the human operator can be observed in Fig 4.13g, 4.13h, and 4.13i. Despite the 3D RL-IBVS exhibits a more stable behavior as compared to the standard IBVS methods, which can be derived from the analysis of the metrics presented in Table 4.3 and the comparison of Fig. 4.13a, 4.13d, and $4.13 \mathrm{~g}$, it should be noted that this comparison in real-flight conditions is difficult to obtain since the reproducibility of the experiment in the exact terms for each trial is difficult to guarantee as the target is carried by a human operator. The human operator has reference points to try to increase the reproducibility of the experiment for each IBVS test, however, inherent errors can appear while moving the target. In addition, it should be emphasized the tuning process of the standard IBVS techniques, which is made by trial and error in real-flight conditions and cannot guarantee that the configuration reached after the tuning process corresponds to the optimal behavior for the specific controller. However, this is a standard procedure in the engineering community, and thus we consider the results obtained throughout the comparison of the different IBVS controllers in realflight conditions as valid, which can reveal important intuitions of the behavior of each IBVS controller and can contribute to the body of knowledge.

The results obtained in real flight experiments for both 2D and 3D RL-IBVS reveal the good simulation-to-real transition capabilities of the proposed RL-IBVS agents, which were completely trained in simulation using the dynamics of the AscTec Hummingbird quadrotor available in the RotorS Gazebo simulator, and tested in real flights using a 
Parrot Bebop 2 and a custom-designed quadrotor. These good transition capabilities are benefited by the appropriate dynamics of the UAVs available in the RotorS Gazebo simulator, and are of crucial interest in the field of aerial robotics, where directly training a reinforcement learning algorithm in a real scenario is very complicated and to the authors' knowledge has not been yet addressed in the literature.

\subsection{Conclusions}

In this chapter, a versatile deep reinforcement learning agent has been efficiently trained for learning 2D and 3D image-based visual servoing policies for object-following missions conducted in simulation and real-flight scenarios.

In order to train and evaluate the proposed RL-IBVS controllers, a reinforcement learning framework has been developed. The proposed RL framework integrates a deep RL agent with an environment built on top of the RotorS Gazebo simulator. In this chapter, the IBVS agent based on the Deep Deterministic Policy Gradients (DDPG) algorithm, has been analyzed and adapted for performing IBVS tasks in 2D and 3D spaces. The 4-layer fully-connected actor and critic neural networks that compose the DDPG while combined with the RL methodology proposed in our RL-IBVS approach have shown to be an effective technique for learning stable IBVS policies.

The most important findings after the experiments conducted and the results obtained throughout this chapter are summarized next:

- The proposed RL framework based on the coordination of a deep RL agent running in Python, and an environment based on the RotorS Gazebo simulator running in $\mathrm{C}++$, and using ROS for communication between both agent and environment, has demonstrated to be an effective framework for learning IBVS controllers. The proposed RL-IBVS controllers act as high-level controllers used for generating linear velocity commands to a standard velocity controller integrated into our Aerostack architecture. Future works aligned with the design of mid-level controllers for commanding the UAV directly in attitude (i.e., roll, pitch, and yaw commands) will be considered for future research directions.

- The proposed reward function, based on the difference between shaping functions in two consecutive time steps, has demonstrated to be an effective strategy for training RL-IBVS agents. In the case of the 2D RL-IBVS, the shaping function consists of an energy function in an L2 fashion based on the errors in the $x$ and $y$ axes with respect to a predefined reference in the image plane. Similarly, the shaping function in the case of the 3D RL-IBVS integrates the shaping of the 2D RL-IBVS and incorporates additional L1 terms to measure the error in diameter (measured in pixels) for inferring altitude commands. Moreover, the stabilization of the image in order to remove the effects of high roll and pitch commands has shown to be a crucial technique in order to train and test the agent. Training the agent using directly the not stabilized detection of the target in the image plane, led to learning unsuccessful policies. 
- The proposed IBVS strategy based on the information of the detected ROI in the image, provides a versatile solution allowing to decouple the object detection stage from the IBVS stage. In this way, the proposed RL-IBVS agents can be adapted to a wide range of applications, where different object detectors can be used.

- Finally, the results obtained in simulation and real-flight experiments, comparing the performance of the proposed RL-IBVS controllers to standard IBVS methods, reveal the outstanding capabilities of partitioned IBVS approaches. Decoupling the velocities generated by the IBVS controller in the $x$ and $y$ directions from the ones generated in the $z$ axis, has provided stable behaviors in all the experiments conducted in this chapter. Similar results were achieved by the proposed RL-IBVS variants, which have shown to provide smooth and stable IBVS policies in simulation and real-flight experiments. It should be noted the almost direct transition of the proposed RL-IBVS controllers to perform real-flight experiments. This easy transition makes the proposed system an alternative solution to classical IBVS controllers, which in general need a hard tuning process of their gains for being adapted to operate in different scenarios. This tuning procedure is even more critical when transitioning from simulation to real-flight scenarios. 



\section{Chapter 5}

\section{Reactive Navigation}

\subsection{Introduction}

Autonomous multirotor navigation through unknown, cluttered indoor scenarios is a complex task which traditionally involves an efficient combination of different components in a traditional robotic architecture such as perception, state estimation, and path planning. The complexity of this task increases when the robotic platform is required to navigate in scenarios containing dynamic obstacles. Navigating in dynamic scenarios requires computationally efficient algorithms capable of adapting the path in real-time when a new obstacle appears on the way of the robot. Traditional motion planning algorithms exhibit some limitations in terms of reactive behavior owing to the computational effort required to re-plan a navigation path.

Moreover, another key aspect in path planning is the representation of the map (e.g., grid-based, polygon-based, etc). Grid-based methods can suffer from imprecise obstacle representations or big memory demands especially when the scenario is substantially large. On the other hand, geometric representations of the map can be more efficient in terms of memory consumption while suffering from a high computational cost when representing complex shaped obstacles. In addition, several traditional path planning algorithms may require a fine-tuning stage of their parameters in order to adapt the algorithm to a previously unseen scenario.

Taking into account the aforementioned constraints, in this chapter we introduce an efficient reactive navigation algorithm based on deep reinforcement learning techniques, which has demonstrated outstanding reactive navigation capabilities in scenarios with static and dynamic obstacles. In this direction, the main contribution of this chapter is the development and experimental validation of a mapless reactive navigation algorithm applied to multirotor aerial robots. For this purpose, we use the RL framework presented in Chapter 4 and analyze the integration of different model-free deep reinforcement learning algorithms. More concretely, we study the Deep Deterministic Policy Gradients (DDPG) (Lillicrap et al., 2015) and the Normalized Advantage Fuctions (NAF) (Gu et al., 2016). Using our RL framework, these deep RL algorithms are successfully trained in simulation and directly transferred to a real aerial robotic platform. One of the key properties of the proposed system is the agent's state definition which integrates the relative distance to a 
predefined goal together with the linear velocities of the aerial robot in the $x$ and $y$ directions, and the laser scan data organized in circular sectors. This representation provides the aerial robot with the ability to deal with obstacles of different shape and size. The second main contribution of this work is related to the training process of the agent. To this aim, we adopt an Artificial Potential Field (APF) formulation in order to design the reward function, which has proven to reduce by a large margin the training process of the agent as compared to similar state-of-the-art approaches. Moreover, the computational cost of the algorithm is reduced to a forward pass through a fully-connected network, which leads to fast avoidance maneuvers. The experiments conducted in cluttered and dynamic scenarios demonstrate the appropriate navigation capabilities of the proposed approach, especially in the presence of obstacles that exhibit sudden movements.

\subsection{Related Work}

Autonomous robot navigation in environments with unexpected obstacles is a key challenge in the development of intelligent mobile systems (Yang et al., 2018, Kumar and Michael, 2017). In most cases, specific goals within the working area should be reached, so it is often not enough for the robot to wonder around while avoiding possible collisions. This problem has been traditionally addressed by deliberative approaches integrating localization, global planning and local planning or reactive control (Siegwart and Nourbakhsh, 2004). In this chapter, we will focus on the latter and refer the reader to LaValle, 2006 for a global planning literature review.

The simplest methods for local motion planning are based on the Bug Algorithm (Lumelsky and Stepanov, 1987; McGuire et al., 2019). Other classic techniques are related to artificial potential field concepts, applying the idea that the goal generates attractive forces while the obstacles create repulsive ones for the robot (Koren and Borenstein, 1991). Elastic band methods (Quinlan and Khatib, 1993) consider that the path provided by a global planner is subject to deformations when objects are encountered along the way. Bubbles are used to simplify the implementation. Alternatively, in narrow cluttered environments the Nearness Diagram representation (Minguez and Montano, 2004) for different divide-and-conquer strategies has provided very good results. Recent works have achieved faster and less oscillatory motion for ground robots (Mujahed et al., 2016).

In aerial robotics, some of the latest approaches create local maps and find free-space corridors online (Liu et al., 2016a; Chen et al., 2016). Dynamically feasible trajectories are generated by applying innovative constraints and methods. In the case of platforms with limited resources, computation time plays a major role. Hence, building complex models of the environment for reactive control may be unaffordable. In a novel contribution, Lopez and How, 2017 presented how using raw point clouds as representation and selecting collision free motion primitives in an intelligent manner resulted in impressive aggressive flights of quadrotor vehicles.

Learning abilities for efficient navigation, which is the main topic of this chapter, is showing great potential too. Not only does this open promising options for end-to-end efficient navigation but also reduces the need for complex parameter tuning. One of the first ideas was about learning to imitate reactive human control of Micro Aerial Vehicles 
(MAVs) from visual features, based on heading commands provided by an expert pilot (Ross et al., 2013). An alternative solution was proposed in Gandhi et al., 2017, where the authors argued that requiring expert data for learning is a significant limitation which hinders generalization, and contributed a dataset of real world crashes for vision-based selfsupervised Deep Learning (DL) of heading control. Real world crashes are undoubtedly useful as negative examples to learn from, but sometimes it is not acceptable to damage the robot in the training phase.

Deep Reinforcement Learning (DRL) has been successfully employed to solve difficult navigation problems by means of trial and error experiences. Going beyond heading commands, in Xie et al., 2017, the authors present a DRL strategy to explore visionbased obstacle avoidance policies. The D3QN architecture was trained in simulation to infer discretized linear and angular velocities from raw images. In Zhu et al., 2017 reactive navigation is pursued towards a visual goal, achieving fast convergence and good generalization results by incorporating target information to the state.

Extending successor-feature-based DRL, Zhang et al., 2017 solve navigation problems in simple maze-like environments as a sequence of related reinforcement learning tasks with four discrete commands as possible outputs. In Mirowski et al., 2016, the authors deal with challenging auxiliary functions such as depth prediction and loop closure detection. In Kahn et al., 2017, the authors proposed a method to combine model-free and modelbased DRL for sample-efficient visual self-supervised learning of continuous actions in the real world. Uncertainty was considered so as to improve safety.

Regarding the use of laser range sensors for learning navigation strategies, Pfeiffer et al., 2017 developed an end-to-end DL approach for goal-based local navigation of mobile robots. The proposed CNN model was trained with data from a global path planner, and good reactions to sudden changes were shown. The authors highlight that for fully operational navigation in complex environments their motion planner should be integrated with a global planner providing intermediate targets. Raw laser data was also utilized in Pérez-Higueras et al., 2018 for training an FCN in order to learn feasible paths to the goal using demonstrations from an RRT* planner as ground truth labels. The output of the FCN was combined with an RRT* planner to enhance the collision-free path. For this purpose, The FCN output was used as a cost function to connect the tree nodes and to guide the sampling process of the RRT* planner.

The work by Tai et al., 2017a is the most closely related to the work presented in this chapter. By means of Asynchronous DDPG, DRL is applied to learn continuous control actions for a non-holonomic differential drive robot from 10 laser measurements and the relative position of the goal. The reward function does not take into account the distance to obstacles, and mostly considers the progress towards the goal, penalizing the behavior only when a collision is produced. As for the evaluation, the approach is trained in simulation and tested both in static simulated and real environments. Comparisons are carried out against ROS Move Base and 10-dimensional Move Base, which employ both a global and a local planner. In contrast, the approach presented in this chapter introduces a potential field based method to guide and improve the learning process and provides enhanced robustness against outliers and flawed measurements, which is especially important in the case of aerial platforms that present roll and pitch. 


\subsection{Target-driven Reactive Navigation through RL}

In this chapter, we extend the analysis of the work in Sampedro et al., 2018a by integrating a new Deep RL agent into our proposed framework. Furthermore, we evaluate the learning-based reactive navigation system by comparing its performance to a baseline path planner.

In this section, we provide a detailed description of the proposed learning-based reactive navigation approach. The proposed method uses the RL framework presented in Chapter 4, which is adapted here for navigation purposes. The modularity of the proposed RL framework (covered in Chapter 4 and 5 ) allows the implementation of new RL agents and environments without the need to re-design the framework. Thus, in this chapter we adapt the DDPG agent presented in Chapter 4 for reactive navigation purposes. Besides, we integrate and analyze the performance of a new deep RL agent (i.e., NAF). Finally, we design a new RL environment which allows the formulation of the reactive navigation problem using the RL paradigm, and provides the communication interface with the RotorS Gazebo simulator.

\section{Reactive navigation architecture}

In this work, we model the problem of reactive navigation by means of a fullyobservable Markov Decision Process (MDP) in which at each time step $t$, the agent being at state $\boldsymbol{s}_{t}$ executes an action $\boldsymbol{a}_{t}$ which causes its transition to a next state $\boldsymbol{s}_{t+1}$ and receives a reward $r_{t}$ from the environment. The aim of the agent is to learn the appropriate reactive navigation policy in order to maximize the expected return $R_{t}=\sum_{i=t}^{T} \gamma^{(i-t)} r\left(\boldsymbol{s}_{i}, \boldsymbol{a}_{i}\right)$ given the reward function $r\left(\boldsymbol{s}_{i}, \boldsymbol{a}_{i}\right)$ and a discount factor $\gamma \in[0,1]$. In order to evaluate the reactive navigation policy, the action-value function $Q^{\pi}\left(\boldsymbol{s}_{t}, \boldsymbol{a}_{t}\right)=\mathbb{E}_{\pi}\left[R_{t} \mid \boldsymbol{s}_{t}, \boldsymbol{a}_{t}\right]$ is utilized, which computes the expected return starting from state $\boldsymbol{s}_{t}$, taking action $\boldsymbol{a}_{t}$, and then following policy $\pi$ afterwards.

Using the previous formulation, the proposed architecture for reactive navigation purposes (see Fig. 5.1) has been designed in order to integrate a reinforcement learning agent (e.g., DDPG, NAF) with an aerial robotics simulator (e.g., RotorS Gazebo), using the Robot Operating System (ROS) (Quigley et al., 2009) for communication between the different components.

As can be seen in Fig. 5.1, the environment receives the raw laser range findings coming from a 2D laser range sensor, and the position of the aerial robot and the goal. Based on the previous information, the environment is in charge of computing at each time step the 12-dimensional or 14-dimensional state (observation) defined by $\boldsymbol{s}_{t}=\left\{e_{x}, e_{y}, v_{x}, v_{y}, s_{1}, s_{2}, \ldots, s_{n}\right\}$, and the reward to be sent to the agent. The first four components of the state consist of the normalized errors in position ( $x$ and $y$ ) computed as the relative distance between the position of the aerial robot with respect to the desired goal, and the $x$ and $y$ linear velocities of the aerial robot. In training mode, the linear velocities of the aerial robot are directly taken from the RotorS Gazebo simulator, while in testing mode (e.g., real flights) they are computed by differentiating the position of the robot. The rest $n$ components $\left(s_{1}\right.$ to $\left.s_{n}\right)$ of the state are based on laser data, and are calculated using the following sequence of computations: 


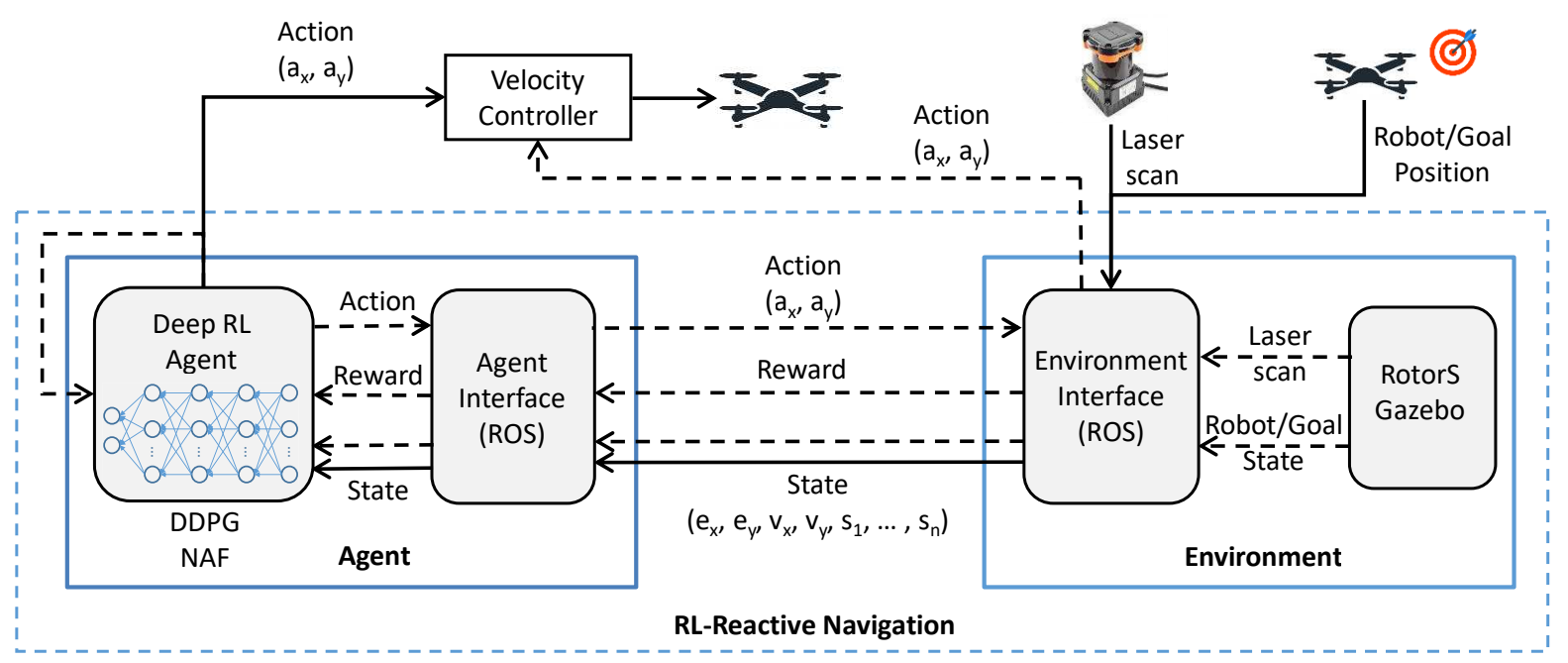

Figure 5.1: Architecture of the proposed RL-Reactive Navigation system. The environment receives as input raw laser range findings, and the aerial robot position from a state estimator module. The agent which implements a deep RL algorithm, receives an observation and a reward from the environment and computes the action (linear velocity in the $x$ and $y$ directions) to be commanded to a velocity controller. The dotted lines represent interactions between the components in training mode (simulation), while the continuous lines depict the interactions in test mode (e.g., real flights).

1. Preprocessing: This step consists of saturating the laser range findings up to a maximum virtual range in order to reduce the influence of the roll and pitch angles of the robot (see Fig. 5.2b). In this chapter, we have considered a maximum virtual range $\left(l_{\max }\right)$ of $2 \mathrm{~m}$, which reduces the field of view of the robot to an area of $4 \mathrm{~m}$ $\times 4 \mathrm{~m}$. This configuration has proven to be crucial in the learning process of the agent, and can provide additional insight of the behavior of the proposed algorithm in platforms that contain sensors with more limited capabilities (e.g., laser range sensor with smaller detectable range).

2. State term based on laser information: Based on the preprocessed laser range findings from the previous step, we divide the whole laser scan into $n$ evenly-spaced circular sectors (see blue lines in Fig. $5.2 \mathrm{~b}$ and 5.2c), where $n$ can take the value of 8 or 10 depending on the configuration analyzed. After that, the average of the normalized laser range findings within each sector is computed (see black lines in Fig. 5.2c) and used as the remaining components in the state $\left(s_{1}\right.$ to $\left.s_{n}\right)$. This method provides a more stable representation of the state, reducing its vulnerability to noisy isolated ranges or possible failures in the sensor. More concretely, every component $s_{i}$ is computed using: $s_{i}=1 / m \sum_{i=1}^{m}\left(l r_{i}-r_{u}\right) /\left(l_{\max }-r_{u}\right)$, where $m$ is the number of laser ranges within each circular sector, $l r_{i}$ corresponds to the laser range $i, l_{\text {max }}$ is maximum virtual range, and $r_{u}$ is the radius of the aerial robot. The latter is a configurable parameter which can be used to adapt the proposed RL-Reactive Navigation approach for its operation in different aerial robotic platforms. 


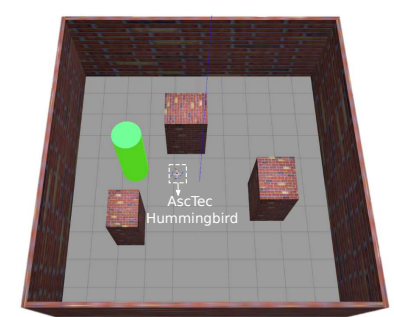

(a)

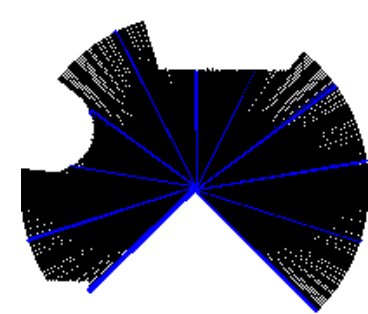

(b)

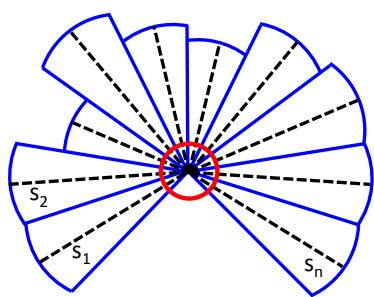

(c)

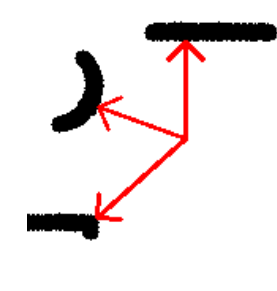

(d)

Figure 5.2: State and repulsive field computation steps (best seen in color). (a) Example of a Gazebo test scenario for illustrating the process. (b) Saturated laser ranges up to a max virtual range. (c) Circular sectors (blue color) and average of the laser ranges within each sector (black lines). The red circle depicts the radius of the robot $\left(r_{u}\right)$. (d) Virtual image generated in order to identify near obstacles, quantify them, and assign them to the corresponding circular sector.

Regarding the agent's side of the proposed RL architecture, in this chapter we have analyzed the performance of two deep reinforcement learning algorithms, the DDPG and NAF. The DDPG algorithm with Batch Normalization is used in order to learn the appropriate policy for generating linear velocity commands to the velocity controller proposed in Pestana et al., 2014. In this work, the actor and critic neural networks of the DDPG consist of fully-connected neural networks with 3 hidden layers of 300 or 400 units each (depending on the configuration). The activation function implemented in each hidden unit is the Rectified Linear Unit (ReLU). The output layer of the actor network is composed of two units with a tanh activation function in order to provide a continuous linear velocity command within the range $[-1,1] \mathrm{m} / \mathrm{s}$, whereas the output layer of the critic network is composed of a single linear unit used to predict the action-value function. Similarly, the NAF algorithm integrates a single fully-connected neural network (not taking into account the target network used in the computation of the loss function), referred to as Q-network (for convenience), which is composed of 3 hidden layers of 300 or 400 units each, depending on the configuration analyzed. In the specific case of the NAF, this neural network predicts the policy from which the actions are extracted, and at the same time provides an estimation of the value function used for the computation of the Q-function. We refer the reader to the corresponding literature and the Appendix A for a more in-depth overview in the formulation of both DDPG and NAF algorithms.

\section{Reward function design}

In order to achieve the two main objectives of our navigation system, that is, reaching a predefined goal while providing an obstacle avoidance behavior, the reward function has been designed taking into account an APF formulation. Thus, the reward function is mainly influenced by two terms which define the attractive and repulsive potential fields.

In order to calculate the attractive potential field, the system relies on a state estimation algorithm which can provide the position of the robot in the world frame of reference. Based on this information and using Eq. 5.1, the attractive potential field is computed based on the Euclidean distance between the position of the aerial robot $\left(\mathbf{t}_{r}\right)$ and the goal 
$\left(\mathbf{t}_{g}\right)$ at the current time step:

$$
U_{a t t}=\alpha \rho_{\text {goal }}\left(\mathbf{t}_{r}\right)
$$

where $\rho_{\text {goal }}\left(\mathbf{t}_{r}\right)=\left\|\mathbf{t}_{r}-\mathbf{t}_{g}\right\|_{2}$ and $\alpha$ is a positive gain and has been empirically obtained to be 100.

Regarding the calculation of the repulsive potential field, we want that each obstacle contributes with only one component to the repulsive field term (see Eq. 5.2). To this aim, we first identify the obstacles in the surroundings of the aerial robot up to the maximum virtual range defined previously. For this, an artificial image is generated (see Fig. 5.2d) by projecting the laser ranges onto an image of predefined resolution $(200 \times 200$ pixels $)$, where the robot is always in the image center. Using this virtual image, we use computer vision techniques in order to find contours in the image which will represent obstacles in the surroundings of the robot. Once the obstacles (contours) have been identified, the distance from the center of the image (aerial robot) to the closest point in each contour is calculated. The vectors formed by the minimum distance to each obstacle in the image (see red arrows in Fig. 5.2d) represent the laser range findings, within the corresponding circular sector, used to compute the repulsive potential field:

$$
U_{\text {rep }}=\beta \sum_{i=1}^{N}\left(\frac{1}{k+l_{i}}-\frac{1}{k+l_{\max }}\right)
$$

where $\beta$ is a positive gain computed using Eq. 5.3, $N$ is the number of detected obstacles at the current time step, $k$ is a constant used to limit the repulsive field and has been empirically set to $0.04, l_{i}$ is the minimum laser range towards the obstacle $i$ within the corresponding circular sector, and $l_{\max }$ is the maximum virtual range value. The last term in the equation $1 /\left(k+l_{\max }\right)$ is used to soften the repulsive field in the instant when new obstacles appear in the field of view of the robot. It should be noted that the term $1 /\left(k+l_{\max }\right)$ allows the definition of an alternative formulation of $U_{\text {rep }}$ in which the summation can be defined up to $n$, that is, the number of circular sectors. In the latter case, the computations involved in the identification of the surrounding obstacles can be omitted since only the circular sectors containing obstacles will contribute to the computation of $U_{\text {rep }}$.

$$
\beta= \begin{cases}\delta & \text { if } \rho_{\text {goal }}\left(\mathbf{t}_{r}\right)>d_{\text {infl }} \\ \frac{\delta}{\exp \left[4\left(d_{\text {infl }}-\rho_{\text {goal }}\right)\right]} & \text { if } \rho_{\text {goal }}\left(\mathbf{t}_{r}\right) \leq d_{\text {infl }}\end{cases}
$$

where $\delta$ is a positive gain and has been empirically found being $\delta=2$, and $d_{\text {infl }}=$ $0.75 l_{\max }$ is the distance of influence from which the repulsive field starts decreasing. This term is used for reducing the influence of the repulsive field when the aerial robot is close to the goal.

Once the attractive and repulsive potential fields are calculated, we compute the reward term using Eq. 5.5) by considering first a shaping function (see Eq. 5.4), which provides information to the agent about its instantaneous progress while speeding up the learning process (Dorigo and Colombetti, 1998). The reward term is finally computed taking into account the evolution of the shaping function in two consecutive time steps:

$$
\text { shaping }_{t}=-U_{\text {att }}-U_{\text {rep }}
$$




$$
r_{t}=\text { shaping }_{t}-\text { shaping }_{t-1}
$$

where $r_{t}$ is the reward obtained at time step $t$, and $U_{\text {att }}$ and $U_{\text {rep }}$ stand for the attractive and repulsive potential fields, respectively. A more compact expression combining Eq. 5.4 and 5.5 can be defined by considering the evolution of the potential field in two consecutive time steps as shown in Eq. 5.6.

$$
r_{t}=\left(U_{a t t_{t-1}}+U_{\text {rep }_{t-1}}\right)-\left(U_{a t t_{t}}+U_{\text {rep }_{t}}\right)
$$

\subsection{Experiments and Results}

In this section, we first describe the experimental setup in Section 5.4.1. Subsequently, the methodology designed for training the proposed RL-Reactive Navigation system is explained in Section 5.4.2. Finally, Sections 5.4.3 and 5.4.4 present the experiments and results obtained in different simulation and real flight scenarios designed for validating the proposed learning-based reactive navigation algorithm. A video of the reported experiments and results is available in: https://vimeo.com/259398134.

\subsubsection{Experimental setup}

In order to train and test the proposed RL-Reactive Navigation system in simulation and real flight scenarios, it has been integrated into the Aerostack framework using ROS as the communication middleware. As explained in previous chapters, Aerostack is an opensource framework for aerial robotics and is used in this chapter to provide additional software components (e.g., state estimator, velocity controller, etc.) which are necessary for the operation of the proposed reactive navigation algorithm and for the development of autonomous missions. In all the experiments, the frequency of the agent has been set to $20 \mathrm{~Hz}$.

Simulation experiments have been conducted using the RotorS Gazebo simulator, in which an AscTec Hummingbird quadrotor has been used as the aerial robotic platform. For training purposes, ChainerRL1 library has been utilized, which is built on top of the Python-based deep learning framework Chainer ${ }^{2}$. Using these libraries, all the models have been trained on an Nvidia GeForce GTX 970 GPU, and tested on an Intel Core i7-6700HQ CPU.

Real flight experiments have been performed in order to validate the capabilities of the RL-Reactive Navigation system in several indoor scenarios with static and dynamic obstacles. The aerial robotic platform utilized for these experiments is the DJI Matrice 100 quadrotor, which is equipped with a DJI Manifold (ARM-architecture) computer and a Hokuyo laser rangefinder UTM-30LX (see Fig. 5.10a). In all the experiments the only information that the robot receives at each time step is the laser scan measurements, limited to the maximum virtual range of $2 \mathrm{~m}$, and the current position of the aerial robot

\footnotetext{
${ }^{1}$ https://github.com/chainer/chainerrl

${ }^{2}$ https://github.com/chainer/chainer
} 
provided by a state estimator. Then, the velocities of the aerial robot in the $x$ and $y$ coordinates are computed by differentiating the position of the former.

\subsubsection{Training methodology}

The simulation scenario used for training the agent is shown in Fig. 5.3 and consists of a $10 \mathrm{~m} \times 10 \mathrm{~m}$ square area with 3 main obstacles inside. The simplistic configuration of the proposed scenario has been purposely designed in order to evaluate the generalization capabilities of the agent in further test experiments.

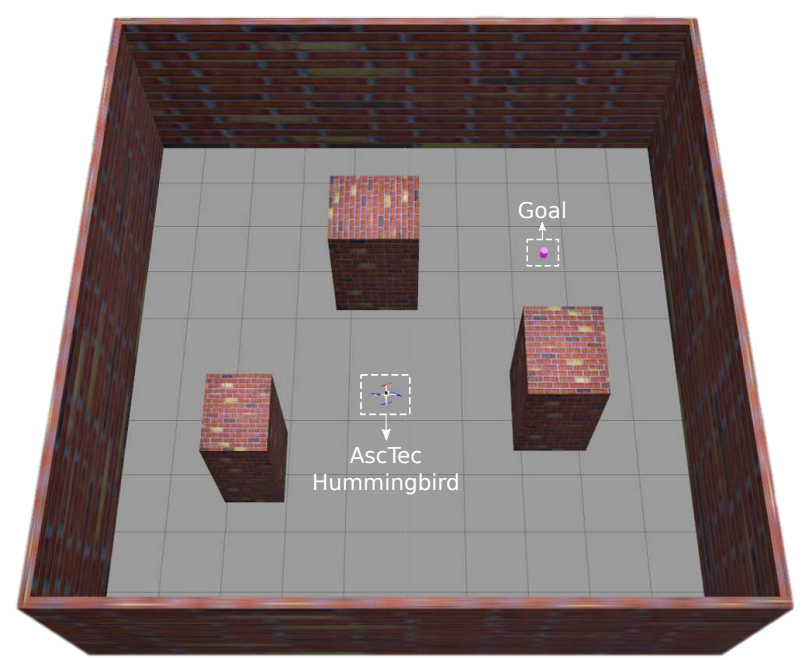

Figure 5.3: Gazebo simulation scenario designed for training the RL-Reactive Navigation system. The pink cylinder represents the goal.

In order to train the aerial robotic agent, the simulation has been configured in an episodic setting, where each episode is composed of a sequence of steps. At the beginning of each episode, the aerial robot and the goal are placed in a random position within the scenario, where the altitude of the aerial robot always remains constant at $1.2 \mathrm{~m}$.

At each time step during the training process, the agent executes an action with added noise according to an Ornstein-Uhlenbeck distribution and receives a reward given by $\mathrm{Eq} 5.5$. This process is repeated until the maximum number of steps (400 steps/episode) is reached or when the aerial robot reaches a terminal state. The latter occurs when the aerial robot collides with an obstacle or when it reaches the goal. In the first situation, a negative reward of -100 is given to the agent, while in the second situation, which represents the main objective, a positive reward of +10 is provided.

During the training process of the DDPG and NAF agents, Adam optimizer (Kingma and $\mathrm{Ba}, 2014$ ) has been utilized with a base learning rate of $10^{-4}$ and a minibatch size of 64 . The rest of the hyperparameters are the same as those presented in the DDPG and NAF original works. In order to select the most appropriate agent, in this work we perform a thorough comparison between three different configurations for each of the deep RL agents considered (i.e., DDPG and NAF) varying the number of neurons in each hidden layer (actor and critic neural networks in the case of the DDPG, Q-network in 
the case of the NAF) and the number of circular sectors that make up the part of the state corresponding to laser measurements. The first configuration studied, referred to as Agent1, has been trained with the actor and critic neural networks, and the Q-network in case of the NAF, being composed of 3 hidden layers of 400 units each. The state considered in Agent1 is a 12-dimensional state with 8 circular sectors as the laser term of the state. The second configuration, referred to as Agent2, has been trained using the same neural networks configuration as in Agent1 and a state composed of 10 laser circular sectors. The final configuration considered, referred to as Agent3, has been conceived as a configuration for analyzing the influence of the number of hidden units in the performance of the agent. Thus, Agent3 has been trained with an actor and critic neural networks, and the Q-network in case of the NAF, composed of 300 hidden units in each hidden layer. In this case, the state has the same configuration as Agent2. Table 5.1 summarizes the three agent's configurations analyzed.

Table 5.1: Configurations analyzed for the DDPG (actor and critic) and NAF (Q-network) agents.

\begin{tabular}{ccccc}
\hline Agent & actor & critic & Q-network & state dim. \\
\hline 1 & $3 \times 400$ & $3 \times 400$ & $3 \times 400$ & 12 \\
\hline 2 & $3 \times 400$ & $3 \times 400$ & $3 \times 400$ & 14 \\
\hline 3 & $3 \times 300$ & $3 \times 300$ & $3 \times 300$ & 14 \\
\hline
\end{tabular}

The training curves obtained after the training experiments for each DDPG and NAF configurations are illustrated in Fig. 5.4, which shows the accumulated reward and actionvalue (Q) per episode. As can be noticed in Fig. 5.4. all the DDPG and NAF configurations analyzed experience an incremental tendency and tend to converge to a similar value which is close to 0 in the accumulated reward (see Fig. 5.4a) and close to $2.5 \times 10^{3}$ in the Qvalue (see Fig. 5.4b). Another important result derived from the analysis of Fig. 5.4 is the training speed of each deep RL algorithm. According to the results presented in Fig. 5.4. the DDPG configurations seem to learn faster than the NAF counterparts. This can be noticed, for example, when analyzing the results of the DDPG agents 1 and 3, which in episode 500 can reach an accumulated Q-value of $2.2 \times 10^{3}$, which practically doubles the result obtained for agents 1 and 3 in the case of the NAF, which achieve an accumulated Q-value of $1 \times 10^{3}$ and $1.3 \times 10^{3}$, respectively.

\subsubsection{Simulation Experiments}

The main objective of the simulation experiments presented in this section is to select the most appropriate agent configuration for the proposed RL-Reactive Navigation system while measuring its generalization capabilities. For this purpose, a benchmark of three Gazebo-based scenarios has been created with different levels of complexity (see Fig.5.5). The first two scenarios (see Fig. 5.5a and 5.5b contain static obstacles of different shapes and sizes, while the last one is made up of dynamic obstacles (see Fig. 5.5c). The last 


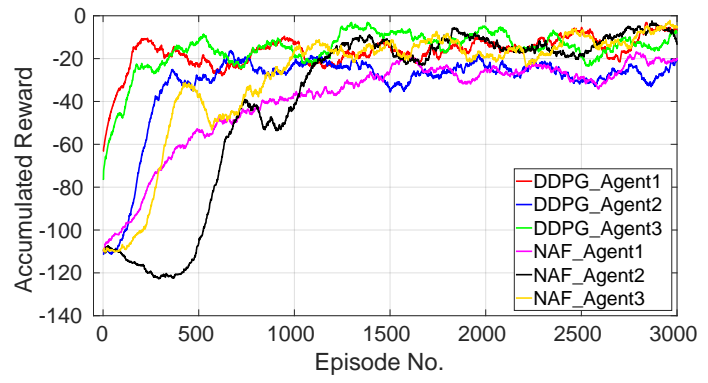

(a)

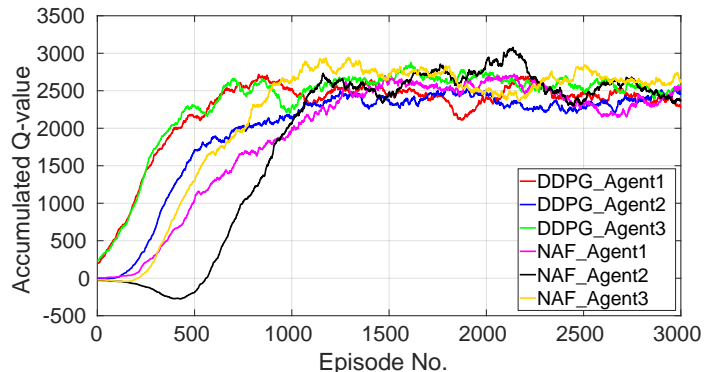

(b)

Figure 5.4: Training curves (best seen in color). (a) Accumulated reward per episode. (b) Accumulated action-value function $(\mathrm{Q})$ per episode. Each curve is depicted considering a moving average of 200 episodes.

designed scenario is of special interest owing to the different type of movement that the obstacles can exhibit (one cylindrical obstacle moves according to a sinusoidal trajectory, while a quadrangular prism obstacle performs sudden movements in front of the aerial robot). It should be noted that in all the simulation scenarios the sizes of the obstacles are smaller than the ones used for training purposes. Furthermore, we introduce static and dynamic cylindrical obstacles which have not been used during the training process. The previous design for the test scenarios is aimed at evaluating the generalization capabilities of the different agents since the states that the agent can reach, especially considering the part of the state based on the laser ranges, are very different from those that it has seen during the training process.

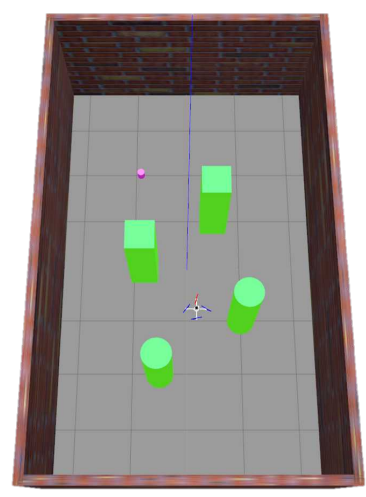

(a)

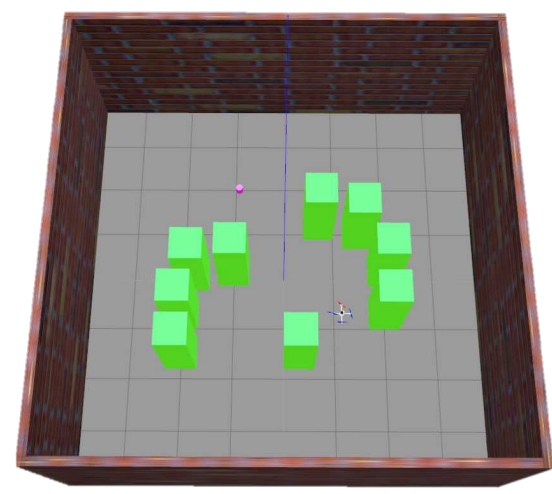

(b)

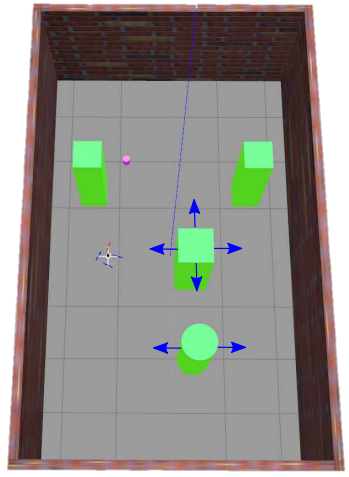

(c)

Figure 5.5: Gazebo simulation scenarios created for evaluating the performance of the proposed RL reactive navigation algorithms. (a), (b) Scenarios containing static obstacles (S1, and S2). (c) Scenario containing two static and two dynamic obstacles (S3). The cylinder obstacle moves according to a sinusoidal trajectory in the $x$ axis, while the moving obstacle in the middle of the scenario performs sudden movements in front of the aerial robot. 
In order to obtain a complete evaluation of the different agents presented in Table 5.1. we compare their performance to a baseline path planner algorithm based on a Rapidlyexploring Random Tree-star (RRT*) which works in combination with a Model Predictive Control (MPC). For a detailed description of the baseline algorithm we refer the reader to Lu et al., 2019. It should be noted that in this case, we use the baseline RRT* as a local planner, which takes the same inputs as the proposed RL-Reactive Navigation system, that is, the laser scan saturated to a maximum virtual range of $2 \mathrm{~m}$. For each reactive navigation algorithm, we perform 100 runs on each of the three simulation scenarios. In each run, the aerial robot is initialized at the bottom part of the scenario while the goal is located at the upper part, at $5 \mathrm{~m}$ distance in the $y$ axis. Both of them are randomly placed within a section of $2 \mathrm{~m}$ in the $x$ axis. In all the runs, the velocity of the aerial robot is limited within the range $[-0.6,0.6] \mathrm{m} / \mathrm{s}$.

The results obtained after the execution of the simulation experiments are shown in Fig. 5.6, 5.7, and 5.8, for the DDPG, NAF, and RRT*, respectively, and summarized in Table 5.2. In this table, four main metrics are shown: $P F$ stands for the performance or percentage of accuracy, which measures the number of tests in which the agent reached the goal successfully. We consider the goal reached when the distance between the agent and the goal is less than $0.3 \mathrm{~m}$. On the other hand, the test is considered failed when the agent collides with any of the obstacles. This situation occurs when any of the laser ranges provides a measurement less than $0.3 \mathrm{~m}$. The variable $P L$ represents the average path length measured in meters, $T G$ is the average time to reach the goal, and finally, $M D$ provides a measurement of the mean minimum distance to the obstacles during the corresponding test. The last three metrics have been computed considering only successful runs, that is, the runs in which the UAV reached the goal.

The results presented in Table 5.2 reveal the good reactive navigation policies learned by the RL agents, highlighting the performance of the DDPG_2 (i.e., the DDPG Agent2) and the NAF_3 (i.e., the NAF Agent3), especially in the third simulation scenario containing dynamic obstacles. Furthermore, most of the configurations involving deep RL agents outperform the RRT* baseline in terms of performance. Besides, when analyzing the time to reach the goal (TG), the results presented in Table 5.2 show that in general the RRT* planner consumes more time to reach the goal, which is more evident in the results obtained for the third scenario where the RRT* obtained a TG of $30.69 \pm 10.32$, which doubles the time required by the DDPG_2 and NAF_3 for accomplishing the same mission.

Regarding the comparison between the RL agents, the results depicted in Table 5.2 show that, in general, the configurations based on the NAF agents outperform those encompassing the DDPG in terms of performance. This can be observed when comparing the performance obtained by Agents 1 and 3. The configuration NAF_1 obtains an average performance in all the scenarios of $87.7 \%$, while the DDPG_1 achieves an average performance of $79.7 \%$. Similarly, the average performance of NAF_3 in the three simulation scenarios is $91 \%$, which outperforms the $79.3 \%$ obtained by DDPG_3. Despite this fact, the best performing configuration is the one obtained by DDPG_2 with an average performance across all the simulation scenarios of 93\%. Agent DDPG_2 has been able to learn a complex reactive navigation policy, which is evidenced in Fig. 5.6h, 5.6i, and 5.6j 


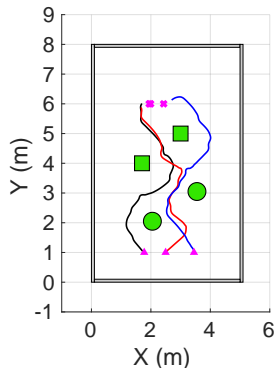

(a)

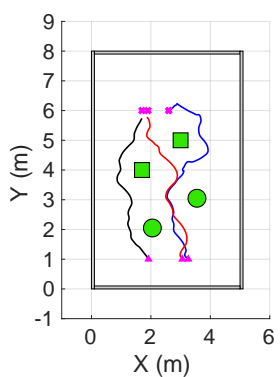

(f)

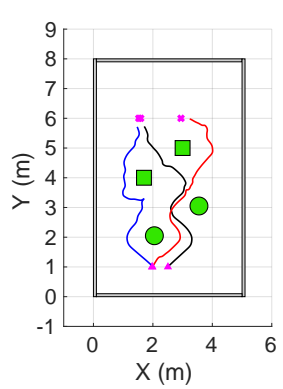

(k)

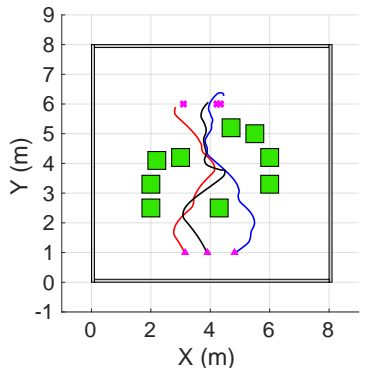

(b)

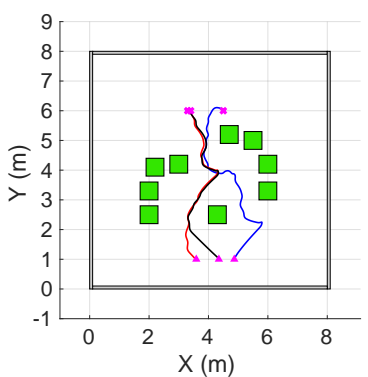

(g)

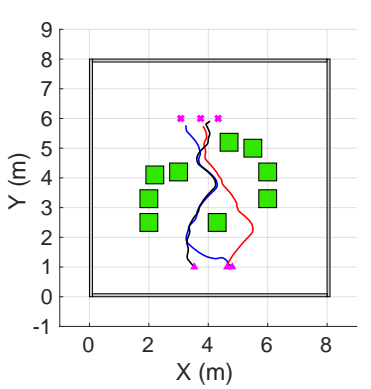

(l)

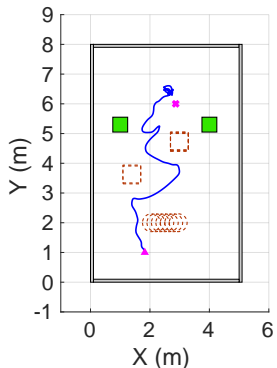

(c)

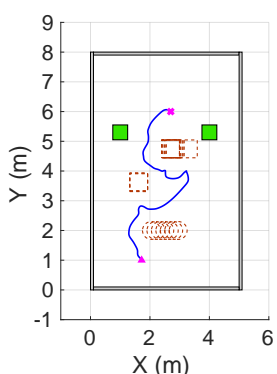

(h)

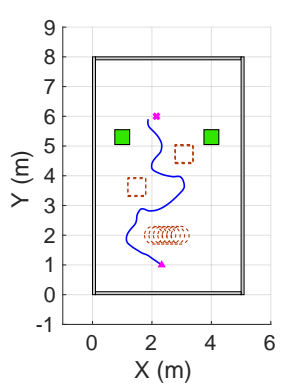

(m)

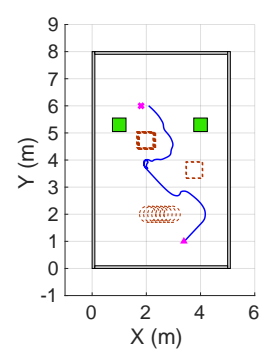

(d)

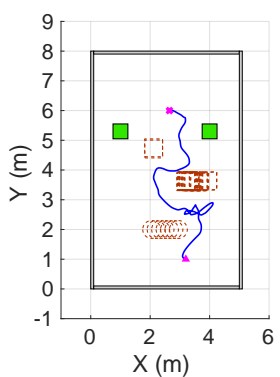

(i)

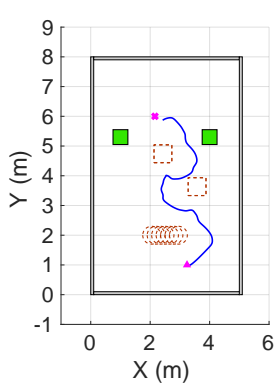

(n)

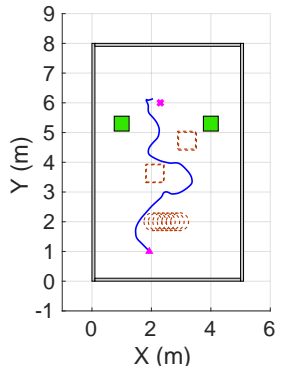

(e)

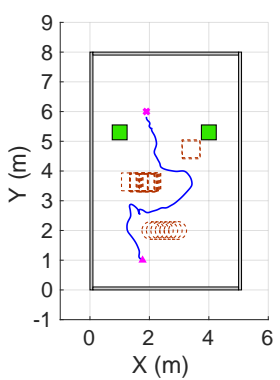

(j)

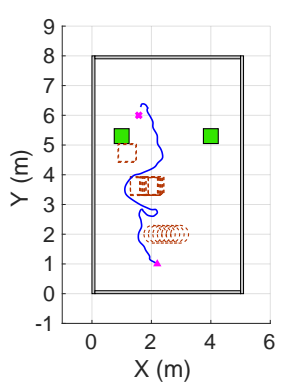

(o)

Figure 5.6: Trajectories generated by the proposed DDPG agents in the simulation flight experiments. (a) to (e) Trajectories generated by Agent1. (f) to (j) Trajectories generated by Agent2. (k) to (o) Trajectories generated by Agent3. The last three figures for each agent contain the trajectories generated in the third scenario involving moving obstacles. The dotted line shapes represent moving obstacles, while shapes with solid lines indicate static obstacles. The pink triangle represents the takeoff point of the agent and the pink cross indicates the location of the goal.

where the DDPG_2 shows safe navigation behaviors that consist of hovering in front of the dynamic obstacle when it appears suddenly in front of the aerial robot, retreating a little to avoid the collision and resuming navigation towards the goal. We refer the reader to the available video, showing the results obtained during the simulation experiments, for better visualization of the navigation policy learned by the DDPG_2 agent.

Despite NAF configurations seem to perform better than the ones based on the DDPG, it should be noted that the navigation behavior of the NAF agents is, in general, more oscillatory than the one learned by the DDPG counterparts. This fact can be observed 


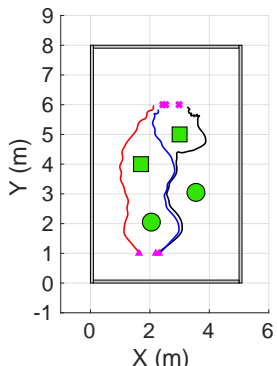

(a)

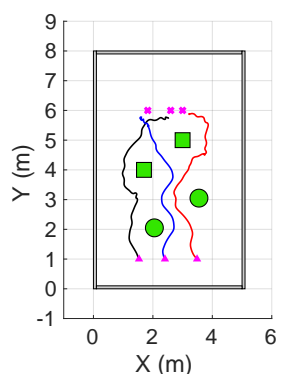

(f)

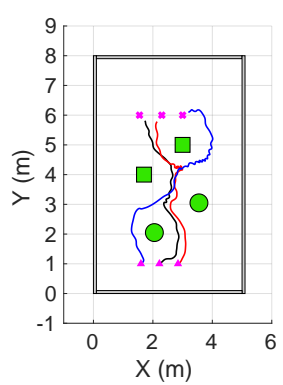

(k)

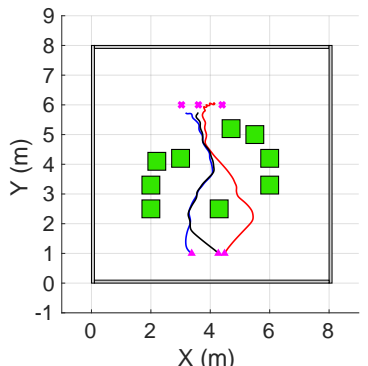

(b)

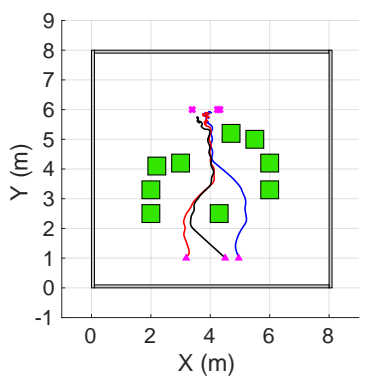

(g)

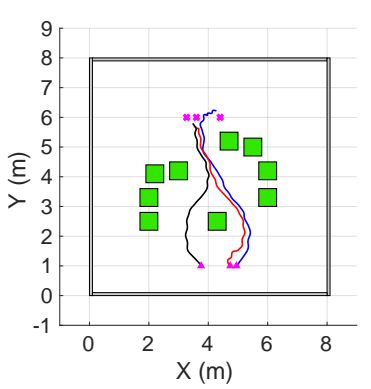

(l)

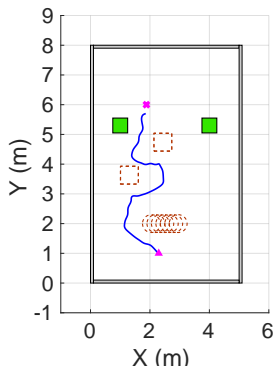

(c)

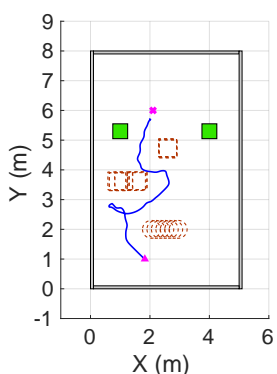

(h)

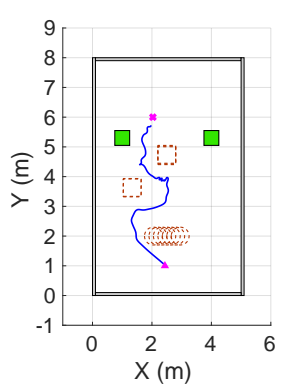

(m)

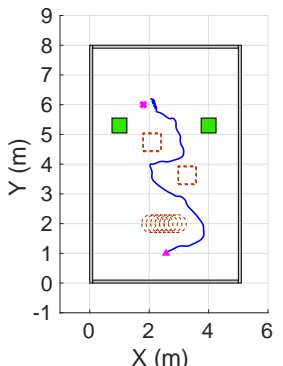

(d)

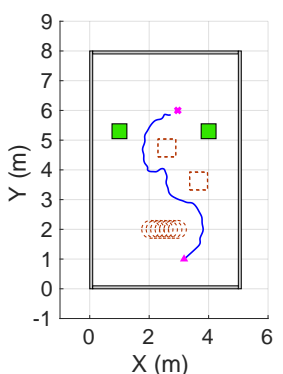

(i)

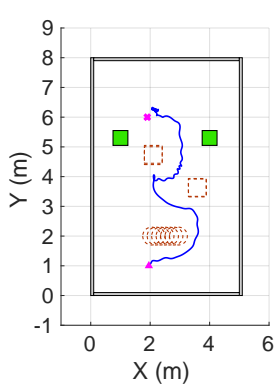

(n)

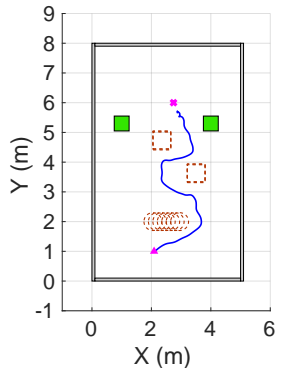

(e)

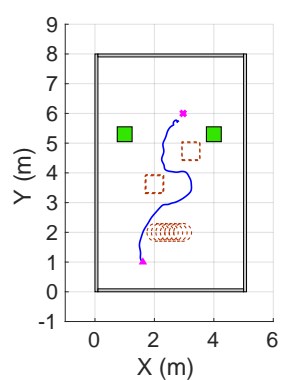

(j)

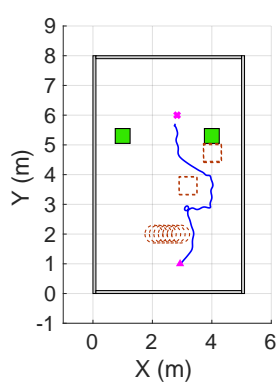

(o)

Figure 5.7: Trajectories generated by the proposed NAF agents in the simulation flight experiments. (a) to (e) Trajectories generated by Agent1. (f) to (j) Trajectories generated by Agent2. (k) to (o) Trajectories generated by Agent3. The last three figures for each agent contain the trajectories generated in scenario 3 involving moving obstacles. The dotted line shapes represent moving obstacles, while shapes with solid lines indicate static obstacles. The pink triangle represents the takeoff point of the agent and the pink cross indicates the location of the goal.

when analyzing the trajectories generated by the DDPG and NAF agents in Fig. 5.6 and 5.7. As shown Fig. 5.7f, 5.7g, 5.7k, and 5.71, the NAF agent starts oscillating at some points of its trajectory. The blue trajectory in Fig. $5.7 \mathrm{k}$ shows an oscillating movement when the UAV tries to avoid the last obstacle towards the goal. Similarly, the blue trajectory generated in Fig. 5.71 shows an oscillating behavior in the area of the goal. These oscillations can be caused by the APF formulation while defining the reward function of the agent and will be further analyzed in future works. 


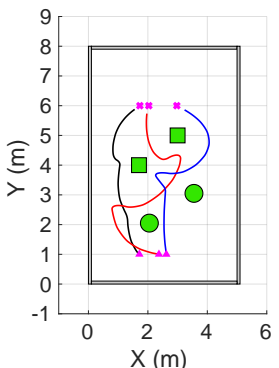

(a)

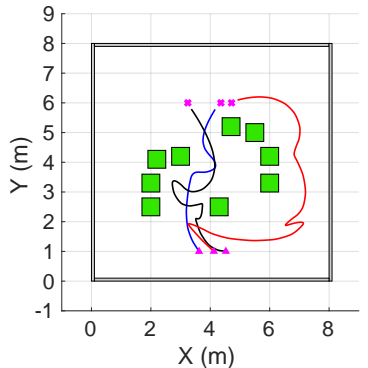

(b)

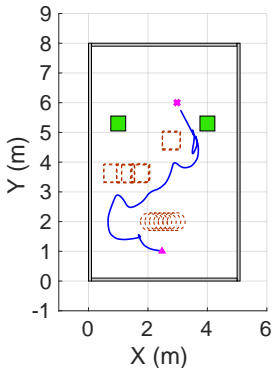

(c)

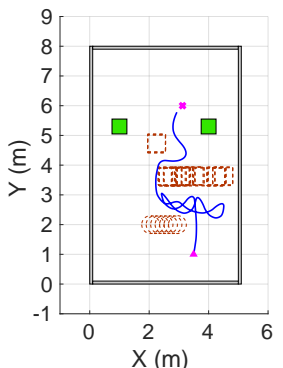

(d)

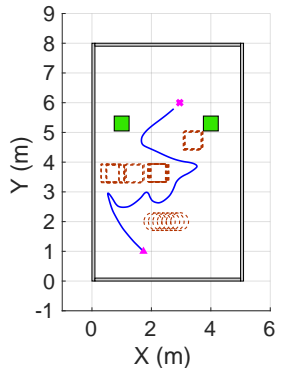

(e)

Figure 5.8: Trajectories generated by the baseline (RRT* + MPC) in the simulation flight experiments. Figures (c) to (e) contain the trajectories generated in the third scenario involving moving obstacles. The dotted line shapes represent moving obstacles, while shapes with solid lines indicate static obstacles. The pink triangle represents the takeoff point of the agent and the pink cross indicates the location of the goal.

\subsubsection{Real-flight Experiments}

In order to further validate the capabilities of the selected DDPG_2 agent in real flight conditions, three experiments of increasing difficulty have been considered. The first experiment has been designed to evaluate the long-term navigation capabilities of the proposed RL-Reactive Navigation system. For this purpose, the objective of the aerial robot is to navigate through an a priori unknown indoor scenario in order to reach a sequence of goals which are defined before takeoff. The scenario consists of an $11 \mathrm{~m} \times 10$ $\mathrm{m}$ area and is composed of static obstacles of varying shape (cylindrical and quadrangular) and size (see Fig. 5.9). Fig. 5.9b shows the trajectory generated by the aerial robot while reaching the sequence of goals.

The same scenario is used in the second experiment, which additionally introduces a dynamic obstacle that is handled by a human operator from within. In this experiment, the moving obstacle tries to block the trajectory of the aerial robot during some period of time in two sections of the scenario. The main objective of this experiment is to evaluate the long-term and reactive navigation capabilities of the proposed system as a whole. Fig. $5.9 \mathrm{c}$ depicts the trajectory generated by the aerial robot while reaching each of the commanded goals. In this figure, sections $A$ and $B$ of the trajectory are highlighted for further discussion, where the aerial robot suddenly encounters the moving obstacle and performs reactive maneuvers in order to avoid it.

Finally, the third experiment has been designed for testing the reactive behavior of the proposed approach in the presence of a fast-moving obstacle. For this purpose, a $4 \mathrm{~m} \times 3 \mathrm{~m}$ area is utilized in which an OptiTrack motion capture system is used for gathering the state of the aerial robot and the moving obstacle (see Fig. 5.10). For a better understanding of the last two experiments involving moving obstacles, we refer the reader to the video demonstration.

In order to accomplish the first two experiments, an additional functionality has been implemented in the RL-Reactive Navigation system, mimicking the functionality of a mission planner. To this aim, and in order to allow the navigation through a sequence of 
5.4. Experiments and Results

Table 5.2: Evaluation results obtained by the different agents in the simulation scenarios of Fig. 5.5. The number after the DDPG and NAF agents depicts the agent configuration.

\begin{tabular}{|c|c|c|c|c|c|}
\hline Scenario & Agent & $P F(\%)$ & $P L(\mathrm{~m})$ & $T G(\mathrm{~s})$ & $M D(\mathrm{~m})$ \\
\hline \multirow{7}{*}{ S1 } & DDPG_1 & 86 & $5.88 \pm 0.40$ & $11.57 \pm 0.85$ & $0.70 \pm 0.03$ \\
\hline & DDPG_2 & 98 & $5.87 \pm 0.24$ & $11.99 \pm 1.44$ & $0.71 \pm 0.06$ \\
\hline & DDPG_3 & 83 & $5.76 \pm 0.13$ & $11.59 \pm 0.78$ & $0.64 \pm 0.07$ \\
\hline & $\mathrm{NAF}_{-} 1$ & 87 & $5.81 \pm 0.12$ & $10.01 \pm 0.77$ & $0.71 \pm 0.05$ \\
\hline & $\mathrm{NAF} \_2_{2}$ & 92 & $6.13 \pm 0.40$ & $13.11 \pm 2.99$ & $0.74 \pm 0.08$ \\
\hline & $\mathrm{NAF} \_3$ & 86 & $6.18 \pm 0.53$ & $13.72 \pm 3.49$ & $0.66 \pm 0.06$ \\
\hline & $\mathrm{RRT}^{*}+\mathrm{MPC}$ & 86 & $5.95 \pm 0.50$ & $15.36 \pm 3.92$ & $0.65 \pm 0.11$ \\
\hline \multirow{7}{*}{$\mathrm{S} 2$} & DDPG_1 & 78 & $6.05 \pm 0.29$ & $11.31 \pm 0.83$ & $0.81 \pm 0.03$ \\
\hline & DDPG_2 & 100 & $5.99 \pm 0.21$ & $12.28 \pm 1.06$ & $0.75 \pm 0.03$ \\
\hline & DDPG_3 & 90 & $5.80 \pm 0.16$ & $10.61 \pm 0.65$ & $0.72 \pm 0.04$ \\
\hline & $\mathrm{NAF}_{-} 1$ & 100 & $5.92 \pm 0.25$ & $10.67 \pm 1.65$ & $0.77 \pm 0.03$ \\
\hline & $\mathrm{NAF} \_2$ & 100 & $6.19 \pm 0.44$ & $13.90 \pm 3.36$ & $0.70 \pm 0.05$ \\
\hline & $\mathrm{NAF}_{2} 3$ & 100 & $5.95 \pm 0.25$ & $10.40 \pm 1.04$ & $0.76 \pm 0.03$ \\
\hline & $\mathrm{RRT}^{*}+\mathrm{MPC}$ & 79 & $6.13 \pm 0.58$ & $18.03 \pm 4.92$ & $0.73 \pm 0.08$ \\
\hline \multirow{7}{*}{ S3 } & $\mathrm{DDPG}_{-} 1$ & 75 & $6.28 \pm 0.47$ & $14.10 \pm 2.02$ & $0.73 \pm 0.07$ \\
\hline & DDPG_2 & 81 & $6.88 \pm 0.81$ & $16.30 \pm 3.49$ & $0.73 \pm 0.06$ \\
\hline & DDPG_3 & 65 & $6.25 \pm 0.52$ & $13.78 \pm 3.15$ & $0.68 \pm 0.05$ \\
\hline & $\mathrm{NAF}_{-} 1$ & 76 & $6.06 \pm 0.28$ & $13.22 \pm 1.88$ & $0.67 \pm 0.07$ \\
\hline & $\mathrm{NAF} \_2$ & 61 & $6.17 \pm 0.37$ & $12.99 \pm 0.96$ & $0.64 \pm 0.06$ \\
\hline & $\mathrm{NAF}_{-} 3$ & 87 & $6.53 \pm 0.44$ & $15.71 \pm 2.81$ & $0.67 \pm 0.08$ \\
\hline & $\mathrm{RRT}^{*}+\mathrm{MPC}$ & 62 & $7.95 \pm 2.29$ & $30.69 \pm 10.32$ & $0.67 \pm 0.06$ \\
\hline
\end{tabular}

goals, we include a simple function inside the RL-Reactive Navigation system which is in charge of managing a list of goals that has been provided to the system before starting the mission. Furthermore, this function is also responsible for checking the distance to the goal in every instant of the mission in order to command the next goal once the current goal has been reached. In addition, in order to adapt the proposed RL-Reactive Navigation to real flight conditions, where the aerial robot utilized (DJI Matrice 100) is bigger than the one used during the training process (AscTec Hummingbird), we make use of the parameter $r_{u}$ defined in Section 5.3. This parameter allows considering the size of the robot and thus provides some flexibility for the adaptation of the proposed system for its operation in different robotic platforms. 


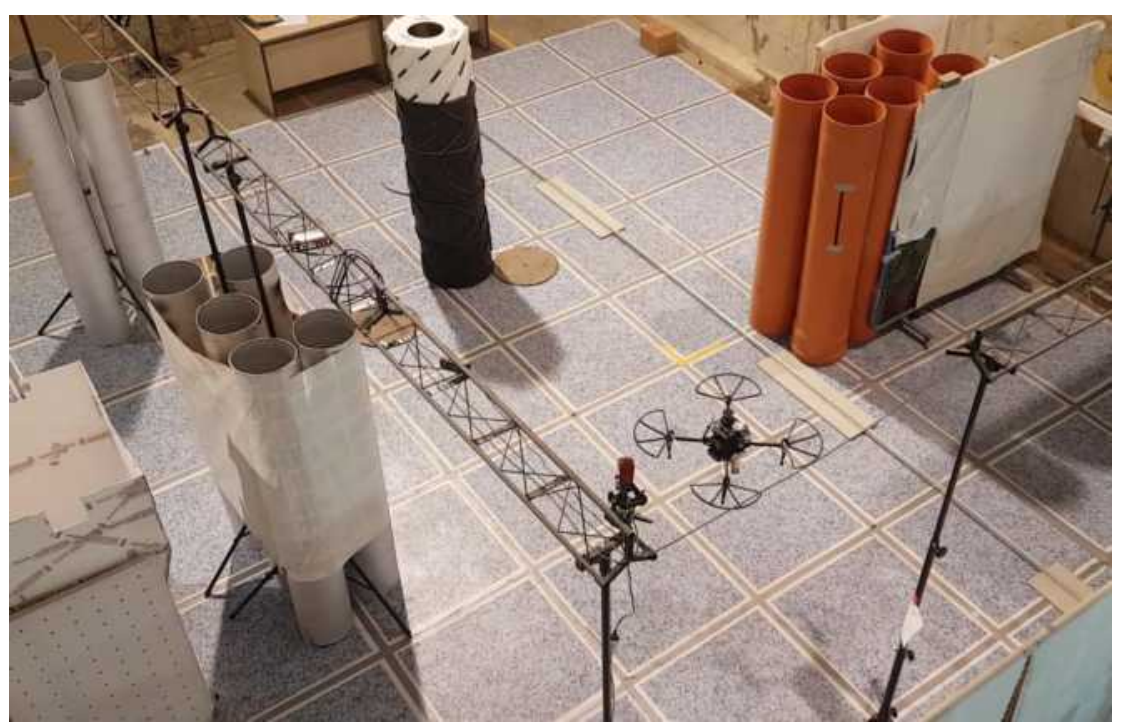

(a)

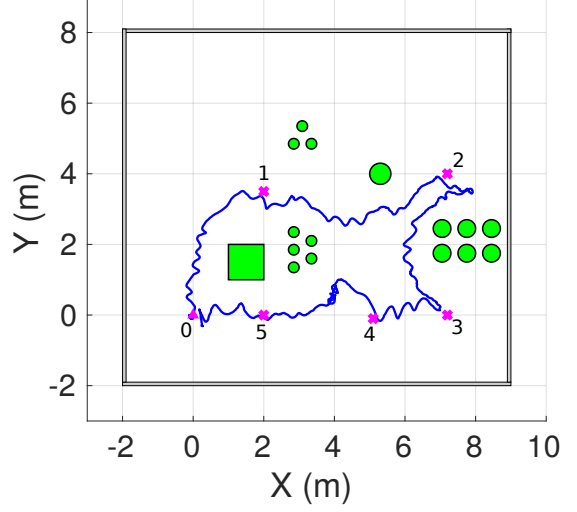

(b)

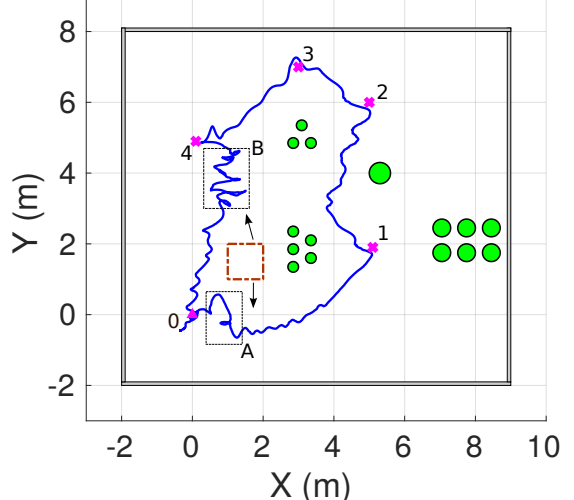

(c)

Figure 5.9: Real Flight experiments designed for evaluating the proposed RL-Reactive Navigation system in long missions with static and dynamic obstacles. (a) Indoor scenario created for the evaluation. (b) Trajectory generated by the aerial robot in the first real flight experiment (static obstacles). (c) Trajectory generated by the aerial robot in the second real flight experiment (static and dynamic obstacles).

The results presented Fig. $5.9 \mathrm{~b}$ and $5.9 \mathrm{c}$ show the appropriate reactive navigation behavior in the presence of static and dynamic obstacles of the proposed RL-Reactive Navigation system, which behaves similarly as in the simulation experiments. In both experiments, the UAV commanded in a fully-autonomous manner by the proposed RLReactive Navigation system, is able to reach all the predefined goals without any collision. A similar stable behavior in more difficult conditions can be seen in Fig. 5.10, where the velocity of the aerial robot exceeds in some cases $1 \mathrm{~m} / \mathrm{s}$. Despite the high velocity reached by the UAV and the sudden movements of the dynamic obstacle in front of the robot, the $\mathrm{UAV}$ is able to accomplish the mission, reaching the predefined goal while maintaining a safety distance to the moving obstacle. 


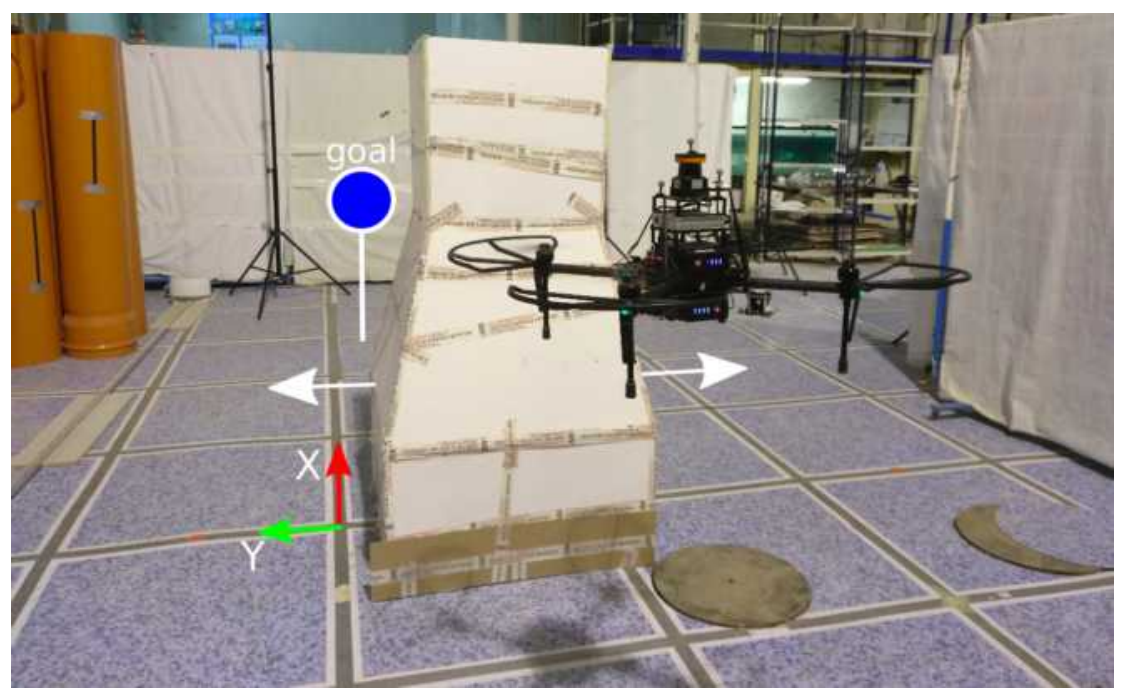

(a)

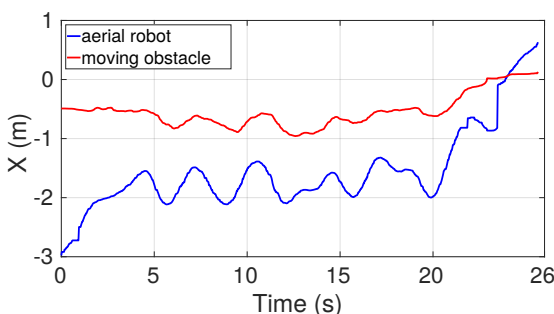

(b)

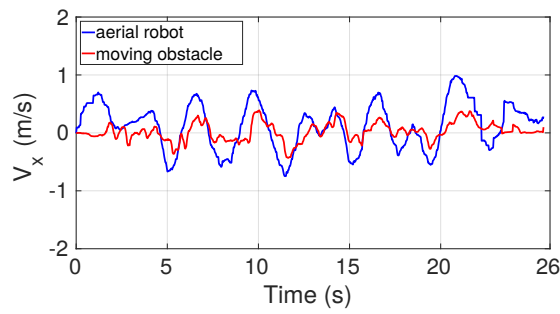

(d)

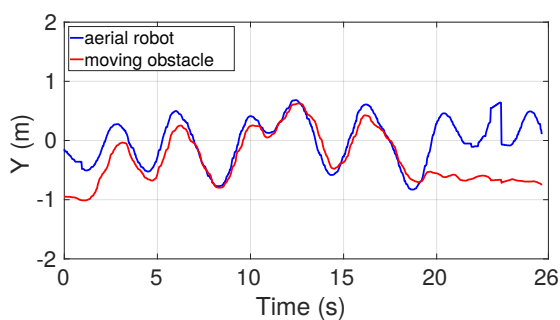

(c)

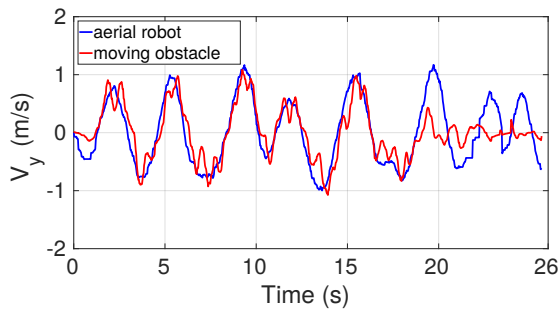

(e)

Figure 5.10: Real flight experiment designed for evaluating the proposed RL-Reactive Navigation system using a fast-moving obstacle (best seen in color). (a) Indoor scenario created for the evaluation. (b), (c) Positions of the aerial robot and the moving obstacle. (d), (e) Linear velocities reached by the aerial robot and the moving obstacle during the experiment.

\subsection{Discussion}

The results presented throughout this chapter for simulation and real-flight experiments demonstrate the appropriate reactive navigation capabilities of the proposed system based on deep reinforcement learning. The proposed system, referred to as RL-Reactive Navigation, integrates an RL environment based on the RotorS Gazebo simulator together with an RL agent which is based on a deep reinforcement learning algorithm. In this chapter, several deep RL algorithms (i.e., DDPG and NAF) have been trained and evaluated for reactive navigation purposes. 
The results obtained after the training process of the RL agents are shown in the training curves depicted in Fig. 5.4 which shows the accumulated reward and Q-value per episode. The incremental tendency and subsequent convergence of the training curves reveal that all the DDPG and NAF configurations are able to learn appropriate reactive navigation policies. Despite all the configurations analyzed tend to converge to a similar value which is close to 0 in the accumulated reward curve (see Fig. 5.4a) and close to $2.5 \times 10^{3}$ in the accumulated Q-value (see Fig. $5.4 \mathrm{~b}$ ), the DDPG configurations exhibit faster learning capabilities. This fact can be noticed around episode 300 in the accumulated reward curve, where the accumulated reward reached by agents 1 and 3 in the case of the DDPG has an approximated value of -20 which greatly differs from the accumulated reward of -70 reached by agents 1 and 3 in the case of NAF. A similar effect can be observed when analyzing the results around episode 500 in the Q-value curve, where the DDPG agents 1 and 3 obtain an accumulated Q-value of $2.2 \times 10^{3}$. In contrast, the same configurations (agents 1 and 3) studied for the NAF agent reach an accumulated Q-value of $1 \times 10^{3}$ and $1.3 \times 10^{3}$, respectively. This fast convergence is benefited by the formulation of the reward function shown in Eq. 5.6. Unlike similar approaches of the state-of-the-art (Tai et al., 2017b) in which the agent is rewarded negatively only in the moment of collision, the inclusion of the repulsive field term allows for providing feedback regarding the proximity of obstacles in each time step. This fact considerably reduces the training time of the agent. In the case of the DDPG configurations, the agent is able to learn an appropriate reactive navigation policy in 600 episodes, taking approximately 147k simulation steps (2 hours).

Regarding the simulation flight experiments, the results shown in Table 5.2, and the trajectories depicted throughout Fig. 5.6, and 5.7, reveal the outstanding reactive navigation policies learned by the deep RL agents in the three Gazebo simulation scenarios containing static and dynamic obstacles. The results shown in Table 5.2, reveal that, in general, the NAF configurations are able to learn safer reactive navigation policies which can be derived from the average performance across the three simulation scenarios of Table 5.2. In this regard, agents 1 and 3 in the case of the NAF obtain an average performance of $87.7 \%$ and $91 \%$, respectively. On the other hand, agents 1 and 3 in the case of the DDPG achieve an average performance of $79.7 \%$ and $79.3 \%$, respectively. However, the results presented in Table 5.2 regarding the time to reach the goal (TG), reveal the higher time required by some NAF configurations to accomplish the mission. As an example, considering the results obtained in the first scenario (S1), agents NAF_2 and NAF_3 obtain a TG of $13.11 \pm 2.99$ and $13.72 \pm 3.49$ seconds, respectively. In contrast, the TG obtained by their DDPG counterparts is 1-2 seconds faster. The higher TG obtained by the NAF configurations is mainly caused by a more oscillatory behavior exhibited at some points of their trajectory, which is further evidenced in Fig. $5.7 \mathrm{f}$. 5.7g, 5.7k, and 5.71, for Agents 2 and 3. As shown in the previous figures, some trajectories present oscillations at different points of the scenario, mainly produced when the agent faces the last obstacle towards the goal (see blue trajectory in Fig. $5.7 \mathrm{k}$ ) and when the agent is exactly in the goal area (see blue trajectory in Fig. 5.7l). This oscillation can be due to the APF formulation, however this effect is not evident in the case of the DDPG agents which generally present smooth trajectories (see Fig. 5.6), and thus will be considered as a future research 
topic. Finally, the best performing agent in terms of performance is the DDPG_2, which obtains an average performance of $93 \%$ across the three simulation scenarios. As shown in Fig. 5.6h, 5.6i, and 5.6j, this agent shows complex reactive navigation behaviors, which in some cases consist of a hard braking when an obstacle appears suddenly in front of the aerial robot. These learned maneuvers lead to a safer behavior which is translated into a higher performance, sacrificing the time to reach the goal. This can be observed in Table 5.2, where the DDPG_2 exhibits the highest TG as compared to the other DDPG variants (i.e., DDPG_1 and DDPG_3). The results presented in Table 5.2 also reveal that, Overall, the agents involving a 14-dimensional state (e.g., DDPG_2 and NAF_3) exhibit higher performances as compared to their 12-dimensional counterparts. Taking these results into account, and based on the average performance obtained by DDPG_2, this agent was selected for conducting real flight experiments.

As a conclusion from the results obtained in the simulation experiments, it should be noted that the proposed deep RL agents provide higher performances and faster reactive navigation policies as compared to the baseline algorithm analyzed in this chapter (RRT* in combination with an MPC trajectory controller). The latter obtains an average performance across the three simulation scenarios of $75.7 \%$, and a TG which in some scenarios (e.g., scenario 3) is almost 2-3 times slower than the one obtained by the deep RL agents. This fact reveals that deep RL algorithms for reactive navigation can be an appropriate alternative solution to classical state-of-the-art algorithms.

Regarding the real flight experiments, the actor network learned by the DDPG_2 (i.e., DDPG Agent 2) has been used for conducting the real flight experiments using a DJI Matrice 100 quadrotor. This network consists of a 5-layer fully-connected neural network of 14-400-400-400-2 neurons (considering the input an output layers) which is fed by the 14-dimensional state consisting of the normalized errors in position with respect to the goal, the $x$ and $y$ linear velocities of the aerial robot computed by differentiating its position, and 10 laser circular sectors, as explained in Section 5.3 . These last terms of the agent's state involving the laser circular sectors are computed by adjusting the parameter $r_{u}$, which takes into account an estimated value for the radius of the robot. The results presented in Fig. 5.9 reveal the outstanding long-term and reactive navigation capabilities in scenarios with static and dynamic obstacles. The reactiveness of the proposed system in real flight conditions is validated in the experiment presented in Fig. 5.9c, where a dynamic obstacle executes sudden movements in front of the aerial robot in sections $\mathrm{A}$ and B of its trajectory. Section B of the trajectory is of particular interest since the dynamic obstacle executes fast movements in the $x$ axis, trying to block the trajectory of the UAV towards the goal. Despite these difficulties, the UAV commanded in a fullyautonomous way by our proposed RL-Reactive Navigation system is able to successfully accomplish the mission, reaching all the commanded goals. A final experiment using a dynamic obstacle executing aggressive maneuvers (velocity greater than $1 \mathrm{~m} / \mathrm{s}$ in some instants of the experiment) in front of the aerial robot is shown in Fig. 5.10. The results presented in Fig. 5.10 reveal the fast reactive navigation capabilities exhibited by the proposed system. The fast response of the system is evidenced in Fig. 5.10c and 5.10e, where the curves showing the position and velocity in the $y$ axis of the aerial robot and the moving obstacle are almost coincident until instant $20 \mathrm{~s}$, where the moving obstacle stops. 
Furthermore, the safety behavior of the system can be observed in Fig. 5.10b, where the UAV always maintains a safe distance $(\approx 1.2 \mathrm{~m})$ from the obstacle until the latter stops and the UAV continues towards the goal.

Overall, it should be emphasized the versatility of the proposed system, which has been completely trained in simulation using a very simple Gazebo training environment employing a quadrotor (AscTec Hummingbird) with different dynamics as compared to the one utilized in real flights (DJI Matrice 100). The outstanding results obtained in simulation and real-flight test scenarios reveal that the proposed learning-based system for reactive navigation constitutes a good alternative to state-of-the-art approaches. The fact that the proposed system has only been trained in simulation opens an interesting research direction, which has special importance in the field of aerial robotics since these platforms cannot be trained in real-flight conditions (or at least to the author's knowledge it has not been yet achieved). Furthermore, the proposed approach is computationally efficient. The inference time to the actor network of the DDPG_2 is approximately $3 \mathrm{~ms}$ measured in an Intel Core i7-6700HQ CPU, and $8 \mathrm{~ms}$ measured in the Manifold computer of the DJI Matrice 100.

\subsection{Conclusions}

In this chapter, a novel 2D laser-based reactive navigation method based on deep reinforcement learning has been presented. The proposed RL-Reactive Navigation system integrates a deep RL agent with an environment based on the RotorS Gazebo simulator. In this chapter, two deep RL agents (i.e., DDPG and NAF) have been thoroughly analyzed and evaluated in different simulation scenarios containing static and dynamic obstacles. Furthermore, the best performing deep RL agent (a 5-layer DDPG) has been validated in several real flight experiments using a DJI Matrice 100.

The most important findings derived after the experimentation conducted in this chapter are summarized next:

- The proposed RL framework presented in Chapter 4 has been successfully adapted in this chapter for designing an RL-Reactive Navigation system able to learn complex reactive navigation policies, what reveals the versatility of the proposed framework for training reinforcement learning agents.

- In order to train the agent, an Artificial Potential Filed formulation has been adopted for defining the reward function. The latter is based on the evolution in consecutive time steps of a function which combines an attractive and repulsive potential fields. Incorporating a repulsive field, which provides information in each time step regarding the proximity of the UAV with respect to the obstacles, has demonstrated to accelerate the training process of the agent as compared to similar state-of-theart approaches. Using the previous formulation, and the RL methodology defined in the proposed RL-Reactive Navigation system, the DDPG and NAF agents are successfully trained in a Gazebo scenario containing static obstacles. 
- The results obtained during the evaluation of the proposed RL-Reactive Navigation system in three Gazebo-based simulation scenarios containing static and dynamic obstacles, reveal the outstanding generalization capabilities of the proposed system. As compared to the training scenario containing only static obstacles, the simulation scenarios for evaluation purposes contain static and dynamic obstacles of different shapes (i.e., quadrangular and cylindrical) and sizes. The best performing deep RL agents, that is, the $\mathrm{NAF}_{3} 3$, and the $\mathrm{DDPG}_{-} 2$, obtained an average performance across the three simulation scenarios of $91 \%$ and $93 \%$, respectively. The appropriate reactive navigation behavior shown by these agents is benefited by the definition of their state, which consists of a 14-dimensional state based on the error in position of the aerial robot with respect to the goal, the linear velocities of the aerial robot, and ten circular sectors resulting from processing the laser scan data provided by a Hokuyo UTM-30LX laser sensor.

- The proposed RL-Reactive Navigation system has been further validated in several real flight experiments in scenarios with static and dynamic obstacles. For this purpose, the proposed system has been integrated onboard the Manifold computer of a DJI Matrice 100 quadrotor. The final actor network extracted from the selected DDPG_2 is used as a high-level controller for commanding linear velocity commands to a standard velocity controller integrated into our Aerostack architecture. This network consists of a 5-layer fully-connected network of 14-400-400-400-2 neurons, which provides extremely fast reactive navigation commands $(\approx 8 \mathrm{~ms})$, as it involves a forward pass to the network.

Overall, this chapter has presented a versatile reactive navigation system which does not need prior information of the scenario where it has to operate. That is, no need for a prior map of the scenario is required, neither building a map while navigating. Furthermore, the laser scan data is saturated to a maximum virtual range of $2 \mathrm{~m}$ with a double purpose of mitigating the effect of high roll and pitch angles in the state of the agent, and at the same time validate the behavior of the proposed system on platforms that might contain sensors with more limited capabilities (e.g., laser range sensor with smaller detectable range). In order to provide advanced navigation capabilities, the incorporation of yaw information in the state of the agent, and the integration of the proposed reactive navigation system into a global planner will be considered for future works. 


\section{Chapter 6}

\section{Mission Planning}

\subsection{Introduction}

In this chapter, we present a versatile and flexible mission planning architecture for the development of high-level missions in a fully-autonomous way. The proposed mission planning system has been designed for managing a multi-robot system, and thus it can handle the coordination of a swarm of Unmanned Aerial Vehicles (UAVs) for the execution of the mission. In this direction, this chapter presents the final mission planning system, which is the result of an iterative design process over several years (Sampedro et al., 2016 Sampedro et al., 2017; Sampedro et al., 2018b). First, the proposed system was conceived to coordinate a swarm of UAVs for the navigation in indoor scenarios using vision, with the main goal of achieving complex missions (e.g., exploration, find a target, etc.) without human supervision. Subsequently, the capabilities of the proposed mission planning system were enlarged for managing more complex missions, such as Search and Rescue (SAR), which usually involve the interaction with the detected target.

This section highlights the main difficulties that a fully-autonomous system should address for conducting high-level missions in indoor scenarios. Subsequently, we present the main motivations that led to the development of the proposed mission planning system. For this, we focus first on the motivation for the development of a multi-robot architecture, and then we focus on providing the appropriate flexibility to the proposed system in order to handle very complex missions which require efficient coordination between several components in a robotic architecture.

In recent years, unmanned aerial vehicles have received increasing attention from the research community, especially multirotor aerial robots due to their high versatility and maneuverability in cluttered indoor environments. This maneuverability (Verbeke and Schutter, 2018) is crucial for the development of complex missions such as exploration of unknown areas, search and rescue, etc. However, UAV navigation in indoor and unstructured environments is a very challenging task due to the lack of Global Navigation Satellite System (GNSS) information, which imposes an important limitation for localization purposes. Furthermore, in most of the missions conducted in real scenarios, the layout of the area to be explored is unknown, that is, the position of the obstacles is not known in advance, nor the position of the target (e.g., in SAR missions). Thus, it 
is essential to design flexible systems that are able to re-adapt the current mission in order to handle different events that can occur during its execution. To these limitations is added the constraints related to the flight time of multirotor UAVs. Nowadays, due to the current energy supply technologies, the flight time of multirotor UAVs, especially those designed for operating in indoor environments, is limited to dozens of minutes. This endurance can be even lower when the UAV has to carry some payload, which is usually the case in SAR missions (e.g., a medical kit).

Successfully accomplish such complex missions, taking into account the constraints mentioned above, requires systems that can optimize the time needed to accomplish the mission. Missions involving inspection and surveillance, such as exploring areas with radioactivity risks (Martin et al., 2015), inspection of disaster areas (Adams and Friedland, 2011; Michael et al., 2012), power line inspection (Deng et al., 2014), bridges inspection (Phung et al., 2019; Jimenez-Cano et al., 2019), etc., require the use of a sophisticated and coordinated system for optimizing resources while completing the mission. Performing these tasks with only one UAV would require, in most of the cases, several attempts of inspection due to the limitations regarding the flight time endurance. In these scenarios, the use of a swarm of UAVs can lead to the more efficient management of the resources with the consequent savings for the companies. Besides, in certain types of missions where the time of accomplishment is a critical constraint (e.g., SAR missions), a swarm of UAVs can be very suitable to perform the required mission efficiently. However, a swarm of UAVs is a complex system where the different agents need to be efficiently coordinated. For this purpose, the research efforts need to focus on designing a scalable and flexible architecture, capable of managing all the components in such a complex system.

The efficient management of several components in a robotic architecture is a compelling requirement for the development of fully-autonomous systems, both in the management of a single agent and in the coordination of a multi-agent system. Furthermore, achieving fully-autonomous capabilities in such indoor environments requires the research on small-in-size aerial robotic platforms, which pose a significant challenge on the system design, due to their limited payload and computational capabilities. These limitations impose the necessity to develop efficient and lightweight algorithms to find a proper balance between mission performance and computational resources available onboard the aerial robot. This balance becomes especially critical when particularly complex tasks have to be performed by the aerial robot without human supervision since more complex decision-making algorithms are required. The aforementioned constraints are present in most of the SAR scenarios, where the aerial robot usually requires to perform high-level tasks such as exploration of unknown scenarios, navigation with collision avoidance, target detection and interaction, among others.

In this chapter, we address the aforementioned constraints with special attention on SAR missions. For this purpose, our preliminary works using swarms of UAVs (Sampedro et al., 2016) are focused on solving the task of search, where the principal objective is to explore an unknown indoor scenario to find a predefined target using a swarm of UAVs. Subsequently, our recent works (Sampedro et al., 2017; Sampedro et al., 2018b) provide a complete solution for SAR missions, incorporating the capabilities for addressing the rescue task. 


\subsection{Related Work}

In this section, we provide a detailed overview of state-of-the-art works that focus on the development of multi-robot architectures, and on the other hand, we analyze the works that focus on the accomplishment of search and rescue missions.

In recent years, and due to its relatively low cost and high flexibility, swarms of UAVs have been increasingly investigated, and utilized for different types of missions such as search and rescue (Waharte and Trigoni, 2010, Scherer et al., 2015, Cui et al., 2016, San Juan et al., 2018), automatic target detection and recognition (Parunak et al., 2003, Vincent and Rubin, 2004; Dasgupta, 2008), hunting a target employing multiple UAVs (McCune and Madey, 2013), and other high-level missions that require the coordination between several UAVs to perform the tasks in a faster and more efficient way. Several biologically-inspired approaches and strategies have been proposed for controlling the swarm behavior, such as strategies based on pheromones (Gaudiano et al., 2005; Dasgupta, 2008; Alfeo et al., 2018), or using evolutionary algorithms (Gaudiano et al., 2005; Lamont et al., 2007; Boškovic et al., 2009; Ramirez-Atencia et al., 2017).

The interaction between a swarm of UAVs can be seen as a multiagent system where several UAVs perform the tasks with communication and coordination amongst themselves. Thus, research efforts must aim towards the development of versatile and flexible architectures for optimal coordination of a swarm of robotic agents. A decisional architecture was proposed by Gancet et al., 2005, in which several deliberative components were designed for multi-UAV system applications. The authors addressed the difference between operational and decisional autonomy for designing a multi-UAV architecture. In the case of a decisional autonomy, a possible division in several levels was defined, where the higher levels correspond to higher decisional capabilities, such as delegating planning activities to the UAV, mission and task refinements, task re-allocation, etc. Simulation results showed that the proposed decisional architecture was able to deal with high-level decisional requirements.

The work in Gaudiano et al., 2005 used a Genetic Algorithm to evolve swarm control parameters, such as the transition probabilities of a UAV across different modes, the pheromone decay rate, and the pheromone attraction parameters of a UAV for search and suppression of enemy air defense missions. Similarly, in Dasgupta, 2008 the swarming mechanism for automatic target recognition was based on the communication mechanism of insects using pheromones as a positive reinforcement for finding the trail to the target. The results presented were validated only in simulation scenarios (AEDGE simulation platform). In the same line of scope, evolutionary algorithms were investigated in Lamont et al., 2007, where the authors proposed a mission planning system for swarm of UAVs. This system consisted of a combination of several components for mission planning, path planning using multi-objective evolutionary algorithms, a genetic vehicle routing algorithm for vehicle-to-target assignment, and a swarm behavior module that tries to maintain some tendencies for the agents of the swarm such as remain together, maintain safe distance from one another, etc. Again, the proposed approach was tested only in a simulation environment (SPEEDES simulation framework), showing limited dynamic re-planning capabilities and scalability. In Boškovic et al., 2009, the authors 
proposed a six-layered hierarchical architecture, where the problems of real-time mission re-planning, and dynamic agent-to-task assignment were addressed by combining mission planning using evolutionary algorithms, hybrid automata-based task execution and biologically-inspired emergent swarm behaviors.

These biologically-inspired strategies, such as genetic algorithms, present a feasible solution for autonomous mission planning due to their capacity of avoiding computational complexity, obtaining a solution very close to the optimal one. However, the convergence of this kind of algorithms can be very slow due to its unguided mutation, making them less suitable for real-time purposes.

The problem of dynamic mission planning was investigated in Wei et al., 2013. A centralized-distributed framework based on a central controller was proposed, being responsible for the mission assignment to each UAV in the swarm. In order to monitor the mission, the central controller periodically sends status inquiries to all UAVs. More recently, the authors in Liu et al., 2018 proposed a motif-based dynamic mission planning strategy where multidimensional dynamic list scheduling (MDLS) algorithm was utilized for generating planning schemes. Unlike the previous works, in this chapter we present a centralized, dynamically distributed and flexible mission planning architecture based on a Global Mission Planer (GMP) and an Agent Mission Planner (AMP). In the proposed architecture, the AMP inquires the GMP only when needed, e.g., the searched target in the mission is found and a new mission is assigned in response of the event.

Regarding the SAR paradigm, most of the works in the literature have focused on the task of exploration and target identification. Little attention has been put on the interaction with the target once it is detected. Early developments in high-level artificial intelligence applied to aerial robotics were introduced in Doherty and Rudol, 2007. In particular, UAV autonomous missions were implemented for the SAR of injured civilians, with robots being able to scan designated areas, trying to identify injured civilians and attempting to deliver medical and other supplies to identified victims in realistic urban scenarios. The authors in Waharte and Trigoni, 2010 proposed three strategies for search and rescue operations, which were studied and evaluated based on the time to find the target. In the greedy heuristics approach, each UAV moves to the neighboring cell based on the highest belief confidence. In the potential-based approach attractive and repulsive potentials were created for navigating through the obstacles present in the scenario, searching for the target. The Partially Observable Markov Decision Process (POMDP) was studied for creating different observation models and a set of actions for each set of UAVs. The results obtained were presented only in simulation cases of study. The work by Tomic et al., 2012 introduced a modular and extensible software and hardware framework designed for the autonomous execution of SAR missions using aerial robots, which was successfully tested on a quadrotor platform. The intelligent transition from indoors to outdoors was addressed by switching between visual and laser odometry. However, while using multiple sensors (four cameras and a laser scanner), the proposed system did not feature any collision avoidance capabilities.

More recent approaches for SAR operation, make use of deep learning, primarily applied to target identification purposes. In this direction, in the work by Bejiga et al., 2017, a sequence of images of avalanche debris captured by a UAV was processed with 
a pre-trained CNN model to extract discriminative features. A trained linear Support Vector Machine (SVM) was integrated at the output of the CNN to detect objects of interest. Moreover, they introduced a preprocessing method to increase the detection rate and a postprocessing method based on a Hidden Markov Model to improve the prediction performance of the classifier. Experimental results conducted on two different datasets at different levels of resolution showed that the detection performance increased when incrementing the resolution, at the cost of raising the computation time. In Lygouras et al., 2019, the authors used an hexacopter equipped with an Nvidia Jetson TX1 for real-time human detection using YOLOv3. Once the human is detected, the UAV is able to conduct the release of a life-ring to perform the rescue operation. However, only simulation results of the rescue operation were reported. In a similar direction to the previous work, in this chapter we present the work developed for performing rescue operations of a target. The rescue operations of a moving target were addressed in this thesis by using IBVS techniques, that are described in Chapter 4. In this chapter, we present the remaining experiments for SAR missions in unknown indoor scenarios.

\subsection{Mission Planning Architecture}

The proposed mission planning system is composed of a Global Mission Planner (GMP) and an Agent Mission Planner (AMP), which have been integrated into the Aerostack architecture (Sanchez-Lopez et al., 2016, Sanchez-Lopez et al., 2017a). As shown in Fig. 6.1, the GMP (see Section 6.3.1) is integrated into a metacomponent called Society Coordinator, which is in charge of the communication between the robotic agents and the human operator. The AMP (see Section 6.3.2) is integrated into the deliberative layer of each individual robotic agent, acting as an interface between the GMP and the rest of the components in the architecture.

\subsubsection{Global Mission Planner}

The Global Mission Planner (GMP) centralizes the global intelligence of the system in terms of mission planning. This component has been designed for dynamic mission generation and management for a single UAV (Sampedro et al., 2017; Sampedro et al., 2018b) or for a swarm of UAVs (Sampedro et al., 2016). The main functionalities of the GMP are described next:

- Mission generation: The main responsibility of the GMP is focused on the mission generation. For this purpose, its flexible design allows the specification of the mission in two operating modes: manual or automatic. In the manual operating mode, the mission is provided by a human operator who can define the default mission (task by task) to be performed by the UAV (or swarm of UAVs) in an XML-based language. In the automatic operating mode, the GMP interprets a high-level mission command provided by a human operator (e.g., explore, SAR, find target, etc.). Additional inputs in this mode comprise the dimensions of the area to be explored (referred to 


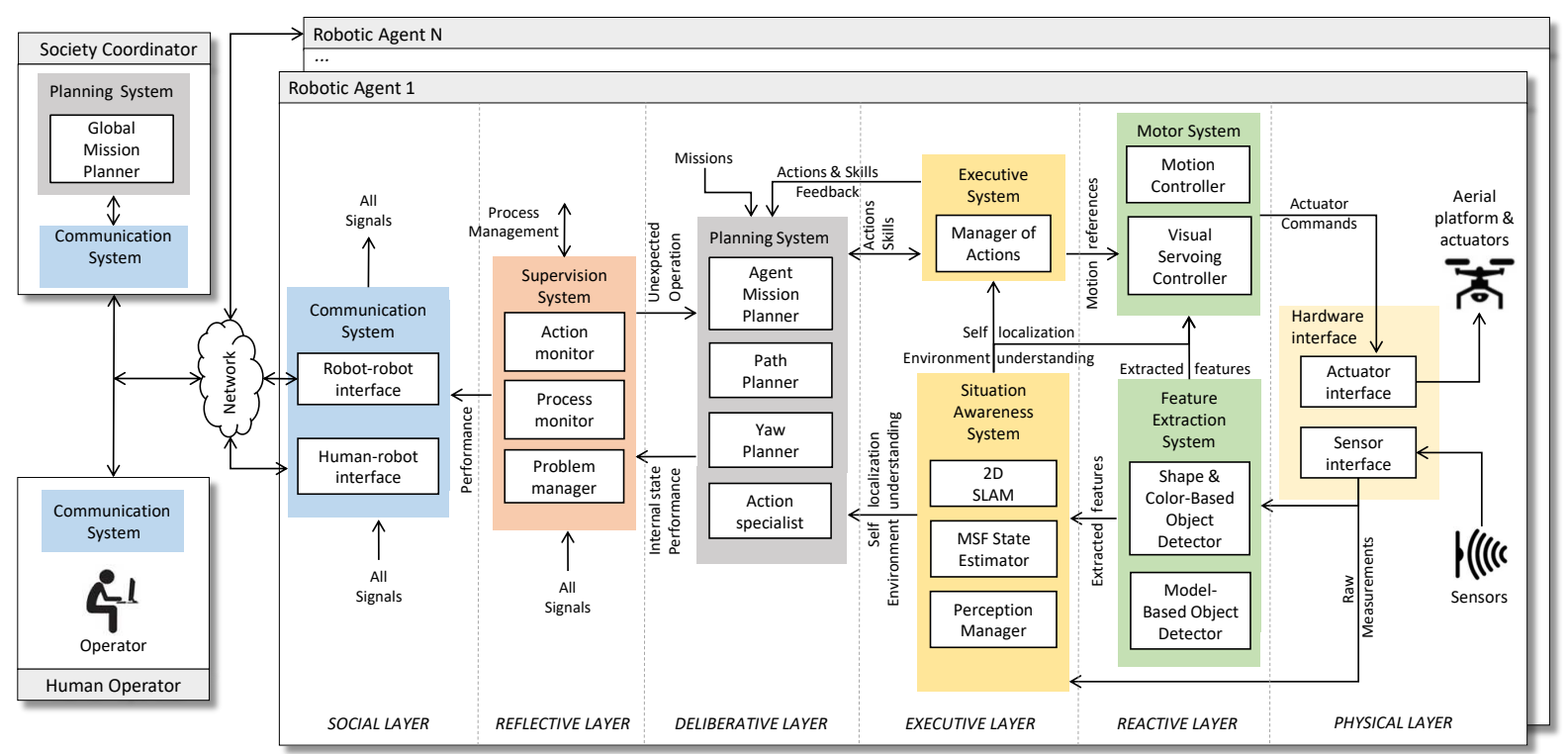

Figure 6.1: Aerostack architecture. The mission planning system presented in this chapter is integrated into the Aerostack architecture in order to perform fully-autonomous missions.

as Mission Zone) as well as the number of mission points. Subsequently, the GMP proceeds as follows in order to automatically generate the mission:

- Sample the Mission Zone and generate mission points: The objective of this step is to divide the area in which the mission has to be performed in several regions. For this purpose, a sampling algorithm based on $k$-means clustering has been utilized. The procedure consists of randomly distributing points through the Mission Zone and perform $k$-means clustering until convergence with the $K$ clusters specified in the configuration file of the mission.

This process is conducted in two steps, the assignment step in which each sample in the Mission Zone is assigned to the closest cluster (see Eq. 6.1), and an update step (see Eq. 6.2), in which each centroid of each cluster is recalculated based on the current members of the cluster. The algorithm converges when the assignments no longer change, satisfying Eq. 6.3 (objective).

$$
\begin{gathered}
\mathbf{C}_{i}^{(t)}=\left\{\mathbf{x}_{p}:\left\|\mathbf{x}_{p}-\boldsymbol{\mu}_{i}^{(t)}\right\|_{2}^{2} \leq\left\|\mathbf{x}_{p}-\boldsymbol{\mu}_{j}^{(t)}\right\|_{2}^{2} \quad \forall j, 1 \leq j \leq K\right\} \\
\boldsymbol{\mu}_{i}^{(t+1)}=\frac{1}{\left|\mathbf{C}_{i}^{(t)}\right|} \sum_{\mathbf{x}_{j} \in \mathbf{C}_{i}^{(t)}} \mathbf{x}_{j} \\
\underset{\mathbf{C}}{\arg \min } \sum_{i=1}^{K} \sum_{\mathbf{x}_{j} \in \mathbf{C}_{i}}\left\|\mathbf{x}_{j}-\boldsymbol{\mu}_{i}\right\|_{2}^{2}
\end{gathered}
$$


Where $\mathbf{C}_{i}^{(t)}$ denotes the set of points assigned to the $i_{t h}$ cluster at iteration $t$, $\mathbf{x}_{p}$ is a point belonging to the Mission Zone, $\boldsymbol{\mu}_{i}^{(t)}$ is the mean (centroid) of the $i_{t h}$ cluster at iteration $t$, and $K$ is the total number of clusters to be computed. Finally, the centroids of the clusters are used by the GMP to generate the corresponding mission points.

- Distribute mission points: This function takes as inputs the mission points calculated in the previous step as well as the take-off point of each UAV in the swarm and generates a list of mission points per UAV. For this purpose, the GMP executes the following sequence of computations:

1. Distribution of the mission points. Each mission point is assigned to the closest UAV in the Mission Zone, according to Eq. 6.4.

$$
\mathbf{U}_{i}=\left\{\mathbf{m}_{p}:\left\|\mathbf{m}_{p}-\mathbf{t}_{i}\right\|_{2}^{2} \leq\left\|\mathbf{m}_{p}-\mathbf{t}_{j}\right\|_{2}^{2} \quad \forall j, 1 \leq j \leq N\right\}
$$

Where $\mathbf{U}_{i}$ denotes a set of mission points assigned to the $i_{t h}$ agent, $\mathbf{m}_{p}$ is the mission point $p, \mathbf{t}_{i}$ is the take-off point of the $i_{t h}$ agent, and $N$ is the total number of UAV agents in the swarm.

2. Arrangement of mission points based on the progressive Euclidean distances along the path. The output points from step 1 (U) are first sorted based on their distances (already computed in step 1) to the corresponding take-off point and then re-sorted using Algorithm 3 for obtaining a feasible path:

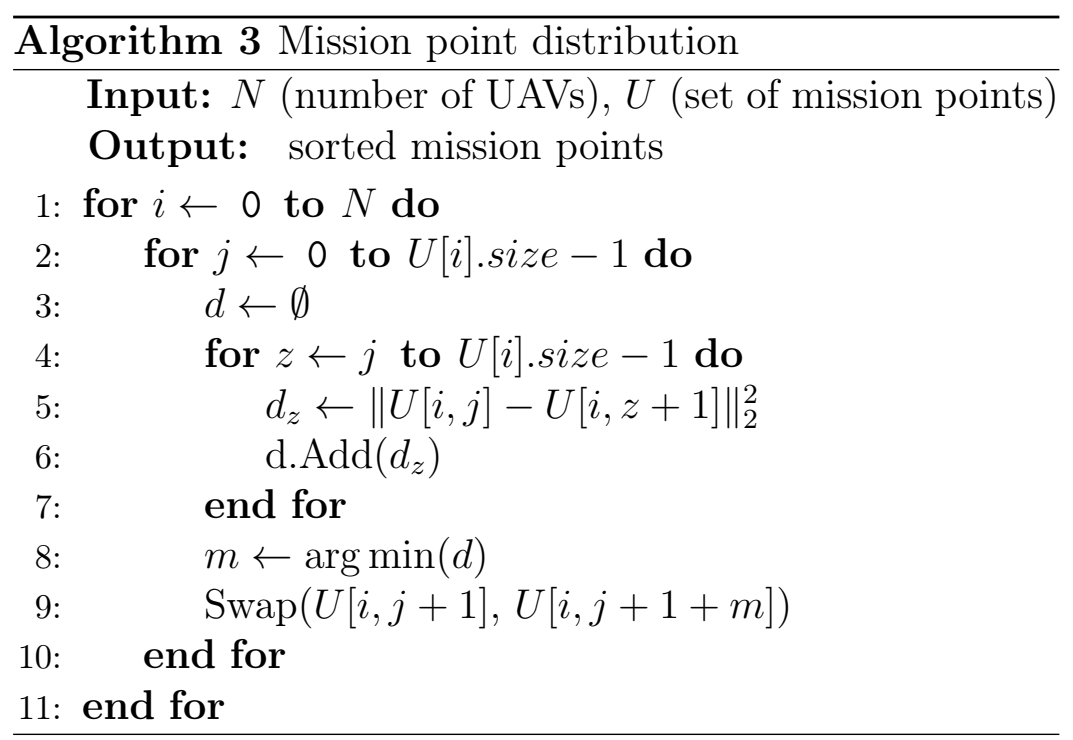

- Generate tasks: Considering the sorted list of mission points obtained in the previous step for each robotic agent, the GMP proceeds by creating the corresponding tasks around these mission points. That is, the mission points generated in the previous step, which are defined by their 3D coordinates in the Euclidean space, are converted into interpretable tasks for the AMP. In the proposed mission planning architecture, all the mission points are translated into tasks called Move To Point. 
Other basic (e.g., Take-off, Hover, Land) and more specific (e.g., TurnInYaw) tasks are pre-defined in the GMP, and are created by the GMP depending on the high-level mission specified by the human operator.

- Generate mission: This step involves the generation of a complete executable mission which can be interpreted and scheduled by the AMP. For this purpose, the GMP incorporates to the previously generated Move To Point tasks, the corresponding tasks for building a complete mission. Additional more specific tasks (e.g., Turn In Yaw tasks in exploration missions) are incorporated in this step depending on the high-level mission defined by the human operator. As an illustrative example, giving 2 tasks of MoveToPoint, a complete mission for a UAV agent in the swarm would be: \{Take-off, Hover, MoveToPoint1, MoveToPoint2, Land\}. Another example involving the same number of mission points for an exploration mission would be: \{Take-off, Hover, MoveToPoint1, $4 \times$ TurnInYaw, MoveToPoint2, 4× TurnInYaw, Land\}. Note that in this more specific mission for exploration purposes, the GMP integrates four Turn In Yaw tasks per mission point.

- Send mission: Once the missions are generated, the GMP sends them to the corresponding AMP of each agent in the swarm.

- Mission re-planning: One of the most important capabilities implemented inside the GMP is the dynamic mission re-planning. In this regard, several predefined behaviors are defined inside the GMP, which are selected depending on the high-level mission that is being currently executed by the system. Based on these predefined behaviors, the GMP is able to dynamically react to some events that can occur during the execution of the current mission (e.g., the target object has been found in a SAR mission, and the rescue operation is triggered).

To achieve dynamic mission re-planning capabilities, one important functionality implemented in the GMP is the possibility of concatenating missions. Based on this, the GMP has an active list of missions per agent (see Fig. 6.2). Once the last mission in the list is accomplished, the GMP is able to recover the previous mission in its corresponding state (i.e., current task). This functionality acquires an utmost importance in SAR missions, where the system has to react to the wide range of events that may occur during the execution of these types of missions. Once an event has been detected, the GMP generates a new mission based on the detected event, thus, saving the state of the previous mission that was being performed and commanding the new generated mission to the AMP.

As an example, if the high-level mission of find target has to be performed, and if one agent in the swarm finds the target, a signal of target found is sent from the AMP to the GMP, which stops the execution of the current mission and assigns a new one to each UAV in the swarm. In the experiments conducted in this chapter (see Section 6.6), and concretely for the find target mission, the re-planning of the mission consists of commanding the UAV that has detected the target to inspect it and sending the rest of UAVs in the swarm to the initial take-off point. Another 
representative example for the accomplishment of SAR missions (see Section 6.7), involves a similar procedure as in the previous example. In this case, once a target is detected, the GMP queues the current exploration mission in order to perform the rescue operation of the detected target. Subsequently, once the rescue mission is finished, the GMP recovers the previous exploration mission in its current state.

\subsubsection{Agent Mission Planner}

This component is located at the agent's level of the architecture (see Fig. 6.1) and is responsible for scheduling task by task the received mission. For this purpose, the AMP acts as an interface between the GMP and the rest of the components in the architecture. We describe next the main functionalities implemented into the AMP component.

- Task scheduling: This functionality involves communication with two main components of the architecture: the Manager of Actions, and the Path Planner. After receiving the complete mission from the GMP, the AMP interprets it and starts scheduling the tasks of the received mission. After interpreting the mission, the AMP starts performing the given tasks of the mission sequentially.

Particularly, once a task of MoveToPoint is scheduled by the AMP, a goal message is sent to the Path Planning system which generates the obstacle-free trajectory to reach the commanded mission point. Once the UAV reaches the current mission point, a goal reached message is sent by the Path Planning system to the AMP, which triggers the scheduling of the next task in the mission.

- Action handling: The AMP acts as a bridge between a high-level mission and the execution of the actions required for the accomplishment of such a mission. During the execution of a task, the AMP translates it into an action (e.g., takeoff, move to a point, etc.), and requests for this specific action to the Manager of Actions (see Fig. 6.2). The Manager of Actions in return translates the requested actions into specific commands for the motion controllers and activates the processes that are needed for the performance of the specific task.

This communication is of particular interest to handle object interaction tasks in the SAR missions presented in this chapter. To this aim, the AMP communicates with the Visual Servoing component, from which it can receive a target locked message when the UAV is located at the desired position with respect to the target in order to start the interaction process (i.e., the rescue operation in SAR missions). Once the AMP receives a target locked from the Visual Servoing component, it communicates with the Manager of Actions in order to send the appropriate command to the corresponding servomotor for releasing the carried object. This means that, when the object of interest (e.g., the container for releasing an object that is being carried by the UAV) is at the desired distance from the UAV, the AMP is the responsible for handling the release action. 


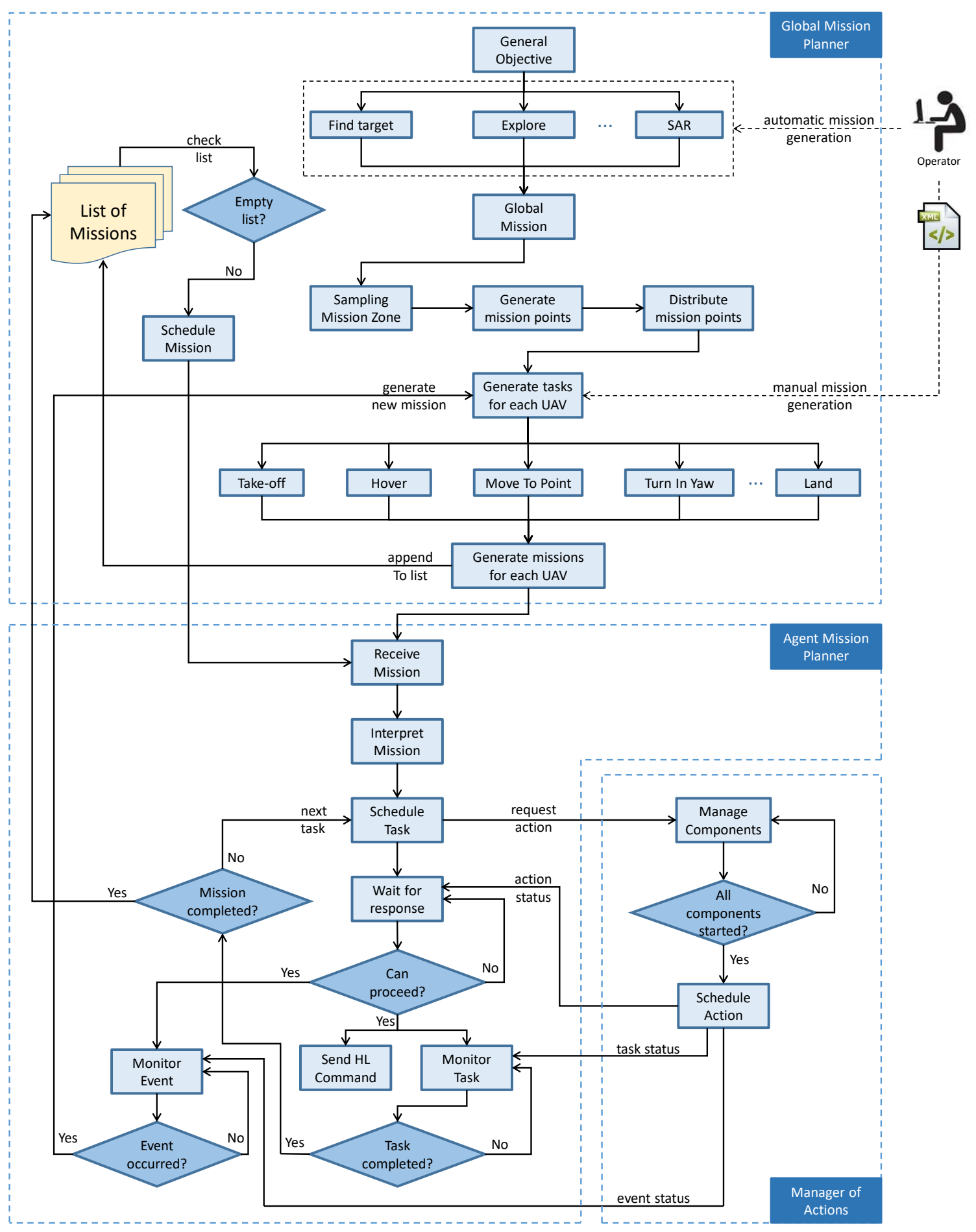

Figure 6.2: Flowchart of the mission planning system for autonomous missions execution.

- Dynamic mission point reallocation: Since the area to be explored is unknown $a$ priori, the mission points generated by the GMP can fall within an obstacle. In 
order to address this problem, the AMP is capable of generating safety points when the current mission point falls within an obstacle. This behavior is activated when the AMP receives a message of goal in obstacle from the Path Planning system. In order to generate a safety point, the AMP implements an iterative method in which random points lying on a safety circumference of predefined radius are generated. After several iterations of the algorithm, and if no safety point has been obtained in the current circumference, its radius is incremented and the iterative method continues. In this iterative procedure, the AMP communicates with the Path Planner in each iteration until an obstacle-free point is obtained.

\subsection{Additional Components}

This section provides a brief description of some additional components integrated into our Aerostack framework and managed by the proposed Mission Planning system in order to conduct fully autonomous missions. We refer the reader to Sanchez-Lopez et al., 2017a; Sampedro et al., 2017; Sampedro et al., 2018b for a detailed explanation of these additional components required to build a fully-autonomous system. In the following paragraphs, the described components are organized according to the different systems defined in the Aerostack architecture presented in Fig. 6.1.

\subsubsection{Planning System}

The GMP and AMP presented in Section 6.3 work in combination with additional motion planning components, which are described next:

\section{Path Planner}

In this chapter, two main path planner components have been used depending on the high-level mission to be executed.

- The path planner component used in the experiments involving swarms of UAVs (see Section 6.6) is based on the algorithm presented in Sanchez-Lopez et al., 2017b. This planner uses geometric primitives (i.e., rectangles and ellipses) to model the objects contained within the scenario to be explored. The sampling strategy used to create the collision-free path is based on a Probabilistic Roadmap (PRM) approach, which takes into account only the information relative to the boundaries of the scenario. Subsequently, an $\mathrm{A}^{*}$ graph-search method, guided by a potential field map as the cost function, is used to find the collision-free path in the graph created by the PRM.

- The path planner component used in the experiments involving SAR missions (see Section 6.7) is based on an existent ROS navigation package $\AA^{1}$, named Navigation Stack (Marder-Eppstein et al., 2010), which relies both on the use of a precise LIDAR

\footnotetext{
${ }^{1}$ http://wiki.ros.org/navigation
} 
sensor and a robust localization and mapping algorithm. This planning algorithm was initially designed for differential-drive and holonomic-wheels robots and was adapted in Sampedro et al., 2018b to be integrated into the Aerostack architecture, enabling its operation with multirotor UAVs. The Navigation Stack package is based on the combination of a global and a local planners. Since the local planner makes use of the kinematics of the robot (ground robot in the original ROS package) to generate velocity commands, in this work we only use the global planner ${ }^{2}$ which is responsible for providing an obstacle-free path using as input a 2D Costmap in which the cells are classified into occupied, free, and unknown. In order to generate the obstacle-free path, the global planner assumes that the robotic platform has a circular shape, and uses an $\mathrm{A}^{*}$ algorithm to plan the path in the Costmap with the obstacles enlarged to propagate the costs of occupied cells using a user-defined inflation radius. Finally, the path provided by the global planner is divided into a sequence of points and passed to our Motion Controller (Pestana et al., 2014) in position control mode.

\section{Yaw Planner}

The yaw planner is in charge of associating a yaw angle to each waypoint within a 2D path based on the direction towards the commanded mission point or considering the AMP directives. Taking into consideration normal mission conditions, a specific policy has been defined:

- Middle waypoints: Orientation is set to a constant value of $\{0, \pi / 2, \pi, 3 \pi / 2\}$, according to the direction of navigation at each time step.

- Last waypoint: Orientation is derived from the AMP directives extracted from the current task within the mission. This can be utilized for exploration purposes, in which different yaw angles can be specified at each mission point.

Following this policy, a UAV can maximize the area covered by both the LIDAR and the image sensor's field of view in order to plan throughout the optimum path and to avoid blind zones which can lead to a collision.

\subsubsection{Executive System}

The executive system is characterized by the Manager of Actions. This component of the Aerostack architecture implements a State Pattern (see Fig. 6.3), which is a behavioral software design pattern that allows the UAV to change its behavior when its internal state changes. Using the state pattern, the Manager of Actions acts as a Finite-State Machine (FSM) responsible for handling the current state of the UAV during the execution of a mission. Furthermore, the Manager of Actions is responsible for managing the components needed for the execution of the current task. For this purpose, the Manager of Actions has a list of components which are supplied by the AMP. This list includes basic components

\footnotetext{
${ }^{2}$ http://wiki.ros.org/navfn
} 
which are indispensable to execute the current task, and additional components which can be provided by the user to provide additional behaviors.

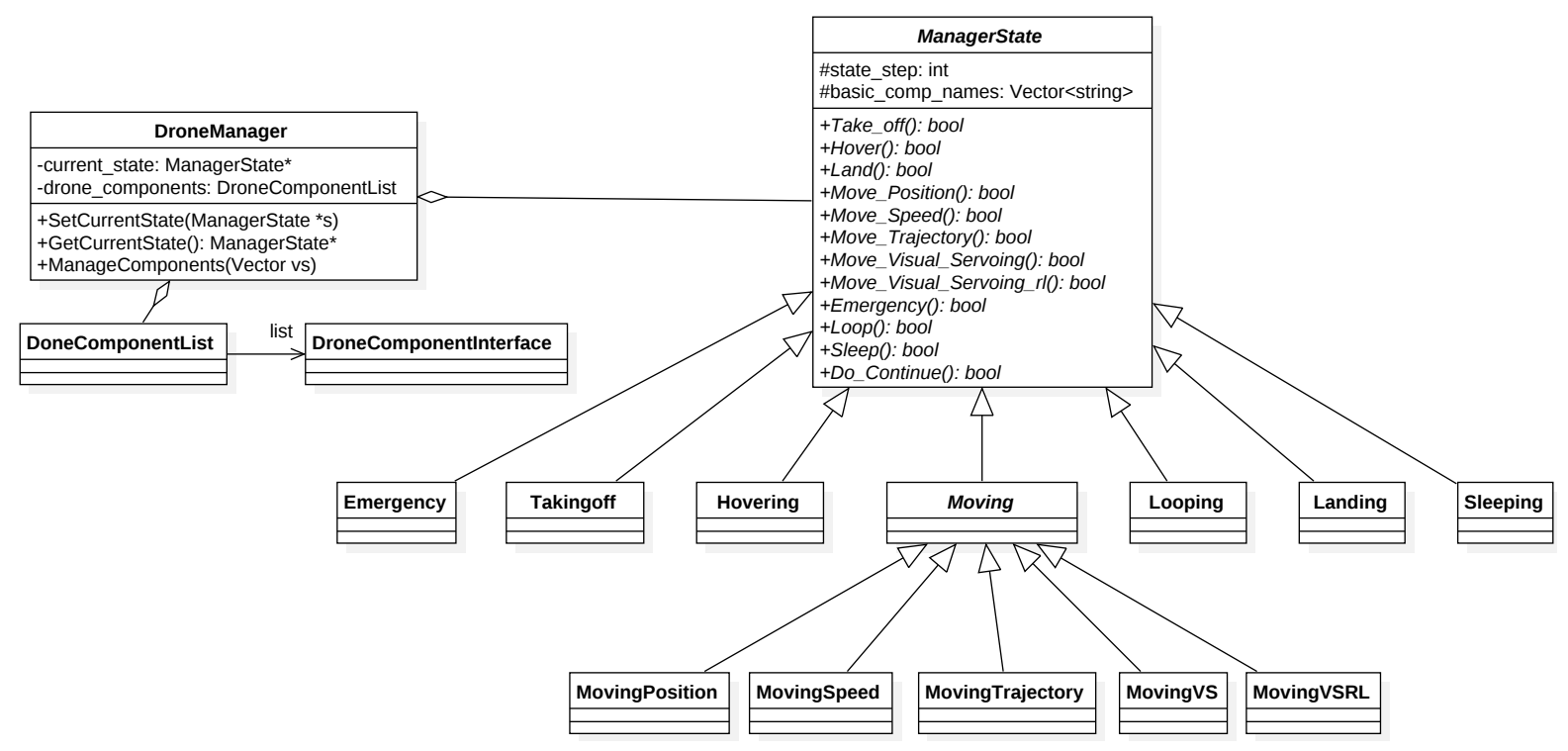

Figure 6.3: UML class diagram showing the State Pattern implemented in the Manager of Actions. Different states are handled by the Manager of Actions, which acts as a finite-state machine.

In order to execute the current task, the Manager of Actions communicates with the AMP, which provides to the former the action that needs to be executed for the accomplishment of the current task together with the list of components. Once the message is received, the Manager of Actions starts handling the activation of all the required components in the list. Once all the required components are started, the Manager of Actions sends the corresponding acknowledgement message to the AMP, which in turn proceeds by scheduling the corresponding high-level command (e.g., sending a goal to the Path Planner) and monitoring the execution of the current task.

\subsubsection{Situation Awareness System}

\section{Localization and Mapping}

As in the case of the Path Planner component, two main algorithms have been utilized for localization and mapping purposes depending on the high-level mission to be executed:

- The SLAM component used in the experiments involving swarms of UAVs is based on the solution presented in Sanchez-Lopez et al., 2015; Pestana et al., 2016. This component combines two main algorithms responsible for SLAM and environment reconstruction. The former SLAM algorithm implements an Extended Kalman Filter (EKF) which is used for fusing the state of the robot based on odometry information with the pose given by the ArUco visual markers located in the scenario. The pose of some visual markers (referred to as landmarks) is known in advance 
and directly used for state estimation purposes. Additional objects (e.g., obstacles) characterized also by a set of ArUco visual markers are used by a mapping stage with a double purpose of estimating the pose of the aerial robot and computing the pose of the obstacles in world coordinates. Finally, an algorithm used for environment reconstruction translates the map previously generated into a map of geometric primitives (i.e., rectangles and ellipses), which is interpreted by the PRM-based Path Planner algorithm for navigation purposes.

- The 2D SLAM component used in the SAR missions, uses the information provided by the Hokuyo laser sensor for localization and mapping purposes. This component is based on a state-of-the-art 2D SLAM algorithm Kohlbrecher et al., 2011, which has been extensively tested for ground robots in Urban Search and Rescue (USAR) missions, and can be also utilized in platforms that exhibit roll/pitch motion by transforming the laser scan into a local stabilized coordinate frame. Using the information coming from a 2D LIDAR sensor, in this algorithm a fast approximation of map gradients, and a multi-resolution grid map representation for mitigating local minima problems, are utilized in order to obtain a stable mapping and a robust scan matching. The latter is computed using a Gauss-Newton optimization process and can be further improved if a source of 3D pose is available and projected onto the $\mathrm{xy}$-plane in order to initialize the optimization process of the scan matcher. The $2 \mathrm{D}$ pose obtained from the scan matching includes the xy-position of the aerial robot and its yaw angle referred in the world frame of reference.

\section{Multi-sensor Fusion State Estimation}

The algorithms presented in this section are only used for the execution of the SAR missions presented in this chapter, and thus work in combination with the Navigation Stack algorithm presented in Section 6.4.1 and the 2D SLAM algorithm described in Section 6.4.3. The objective of the Multi-sensor Fusion State Estimation component is to provide a full 6 DOF pose and the respective velocities of the UAV. In this direction, our proposed architecture integrates two separate state estimation components which can be combined in order to provide a higher level situational awareness functionality or be used separately depending on the requirements of the mission.

- Flight Altitude State Estimator: The 2D SLAM algorithm explained in Section 6.4.3. provides a 2D map of the environment, enabling obstacle detection and avoidance in a 2D plane at the given flight altitude of the UAV. Sensors such as laser altimeters or similar range sensors can lead to errors in the flight altitude estimation when flying above ground obstacles since the measurements get referred to them instead of the ground surface. In order to accurately estimate the flight altitude of the UAV in the presence of several ground obstacles, we use an EKF-based algorithm which is able to estimate the flight altitude of the UAV as well as the elevation of the ground obstacles (more details can be found in Bavle et al., 2017). This is achieved by fusing the measurements coming from the IMU, the barometer, and the laser range altimeter sensor. 
The proposed state estimator considers a state vector $\boldsymbol{x} \in \mathbb{R}^{10}$ based on the combination of four main components: $\boldsymbol{x}_{\boldsymbol{R}}, \boldsymbol{x}_{\boldsymbol{G}}, \boldsymbol{x}_{\boldsymbol{I}}$, and $\boldsymbol{x}_{\boldsymbol{B}}$, which represent the state of the robot, ground object, IMU sensor, and barometer sensor, respectively. The corresponding state of the aerial robot is defined by $\boldsymbol{x}_{\boldsymbol{R}}=\left(\begin{array}{lllll}\boldsymbol{\Omega}_{x y}^{T} & \boldsymbol{\omega}_{x y}^{T} & t_{z_{R}} & v_{z_{R}} & a_{z_{R}}\end{array}\right)$, where $\boldsymbol{\Omega}_{x y}^{T}=(\phi, \theta)^{T}$ are the roll and pitch Euler angles, $\boldsymbol{\omega}_{x y}^{T}=\left(\omega_{x}, \omega_{y}\right)^{T}$ contains the $x$ and $y$ angular velocities of the aerial robot in the UAV frame, and $t_{z_{R}}, v_{z_{R}}$ and $a_{z_{R}}$ are the vertical coordinates of the position, velocity and acceleration of the aerial robot in the world frame of reference. Assuming that the robot changes its vertical acceleration and angular velocity slowly, a constant vertical acceleration and constant angular velocity process model is adopted. The ground object state is defined by $\boldsymbol{x}_{\boldsymbol{G}}=t_{z_{G}}$, where $t_{z_{G}}$ is the altitude of the ground object in the world frame. Obstacles are set so that they always have positive altitude with respect to the ground plane. Finally, the IMU and the barometer sensors contribute to the state with their corresponding biases, thus $\boldsymbol{x}_{\boldsymbol{I}}=b_{a_{z}}$ and $\boldsymbol{x}_{\boldsymbol{B}}=b_{b_{z}}$, where $b_{a_{z}}$ and $b_{b_{z}}$ are the biases in the vertical acceleration and flight altitude measurements respectively.

- Robot State Estimator: This component is able to combine the measurements from the 2D SLAM (see Section. 6.4.3) with the flight altitude estimator or the IMU sensor in order to provide complete pose and velocity estimates of the UAV in the world frame of reference. The Robot State Estimator (Moore and Stouch, 2016) is a standard ROS packag $\AA^{3}$ which implements an EKF-based estimator with state vector $\boldsymbol{x}_{\boldsymbol{R}} \in \mathbb{R}^{12}$, being $\boldsymbol{x}_{\boldsymbol{R}}=\left(\begin{array}{lll}\boldsymbol{\Omega}^{T} & \mathbf{t}^{T} & \mathbf{v}^{T}\end{array}\right)$, where $\boldsymbol{\Omega}^{T}=(\phi, \theta, \psi)^{T}$ are the roll, pitch, and yaw Euler angles, $\mathbf{t}^{T}=\left(t_{x}, t_{y}, t_{z}\right)^{T}$ represents the position of the robot, and $\mathbf{v}^{T}=\left(v_{x}, v_{y}, v_{z}, \omega_{x}, \omega_{y}, \omega_{z}\right)$ is the vector containing the linear and angular velocities of the aerial robot. It includes a non-linear measurement model able to fuse any robot pose or velocity measurements, provided by any number of sensors. In contrast to the Flight Altitude State Estimator, this EKF model does not incorporate in its state vector any biases present in the sensor measurements nor the altitude of the ground obstacles.

The proposed system presented in this chapter, integrates the previously described components for multi-sensor fusion state estimation in a versatile manner, so they can be employed in different configurations depending on the selected hardware and mission requirements at hand.

\section{Perception Manager}

The Perception Manager component is in charge of managing and centralizing the perception events that can occur during the execution of a mission (e.g., object detected, picked and released).

In order to obtain proper management of the perception events, this component integrates the information regarding the current situation of the states of the different objects which the UAV can interact with, together with the own internal state of the Perception

\footnotetext{
${ }^{3}$ http://wiki.ros.org/robot_localization
} 
Manager (e.g., Exploring, Going For Picking Item, Going For Releasing Item). An example of the initial configuration of such states for a SAR mission applied to the use case of IMAV 2016 is provided in Table 6.1. When a perception event is detected by the Perception Manager, (e.g., target detected) the current state of the objects as well as its internal state are evaluated and updated. Based on this evaluation, the Perception Manager can request a mission adaptation event to the GMP via the AMP. As an example, and taking the initial configuration presented in Table 6.1, if a bucket object is detected in the current time instant, and since both corresponding items are already picked up, the Perception Manager will generate a mission adaptation event consisting of Going For Releasing Item. Based on the received event, the GMP will generate a new mission for accomplishing the rescue operation which involves first commanding the UAV to the estimated position of the bucket, and the subsequent visual servoing task in order to release the item inside the bucket.

Table 6.1: Example of the initial state of the objects in a Search and Rescue mission, where $0 / 1$ represents a false/true statement

\begin{tabular}{|c|c|c|c|}
\hline Object State & Detected & Picked & Released \\
\hline Item A & 0 & 1 & 0 \\
\hline Bucket A & 0 & 0 & 0 \\
\hline Item B & 0 & 1 & 0 \\
\hline Bucket B & 0 & 0 & 0 \\
\hline
\end{tabular}

Furthermore, another important functionality implemented in the Perception Manager consists of mapping the objects of interest (e.g., targets) in a SAR mission. For obtaining an adequate mapping of the objects that are being detected during the execution of the mission, the Perception Manager provides a higher level of understanding over the detection events coming from the Model-Based Object Detector component (see Section 6.4.4). For this purpose, the Perception Manager is responsible for referring the relative pose of the detected objects in the frame of reference of the corresponding device (e.g., front camera) to the world frame of reference. This transformation is computed using Eq. 6.5. In addition, in order to provide a robust final pose of the detected objects, the Perception Manager has an internal buffer for storing the pose of the objects being detected over time. This buffer is utilized by a filter in which the poses away from the average pose are removed in an iterative process.

$$
{ }^{W} \boldsymbol{T}_{O}={ }^{W} \boldsymbol{T}_{U} \cdot{ }^{U} \boldsymbol{T}_{C} \cdot{ }^{C} \boldsymbol{T}_{O}
$$

where $\boldsymbol{T}$ stands for a $4 \times 4$ homogeneous matrix transformation. ${ }^{W} \boldsymbol{T}_{O}$ computes the global transformation from world to object frame of reference. ${ }^{W} \boldsymbol{T}_{U}$ is the transformation from world to UAV frame of reference, which is given by the Robot State Estimator component. ${ }^{U} \boldsymbol{T}_{C}$ represents a rigid transformation from UAV to the front camera reference frame, and ${ }^{C} \boldsymbol{T}_{O}$ provides the transformation from camera to object frame of reference, computed online using a Perspective- $n$-Point $(\mathrm{P} n \mathrm{P})$ algorithm (see Fig. 6.4).

\footnotetext{
${ }^{4}$ http://www.imavs.org/2016/
} 


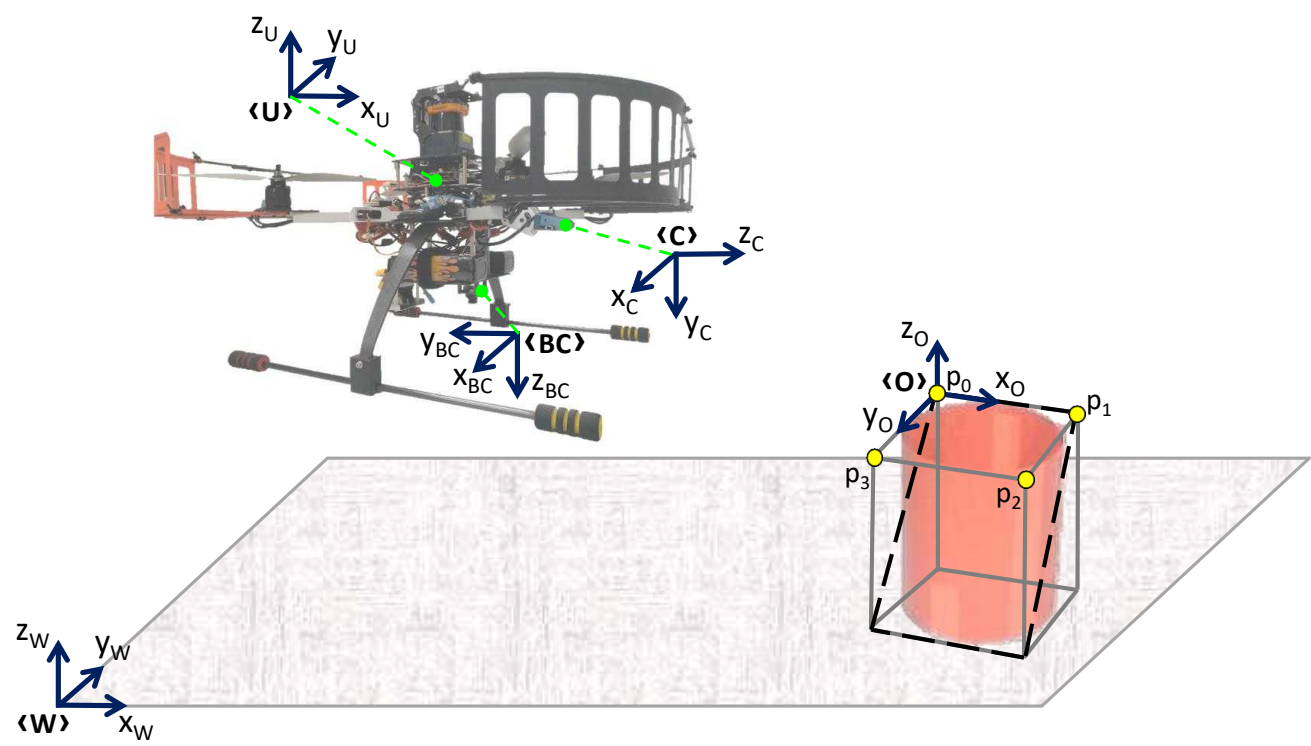

Figure 6.4: Frames of reference defined for the execution of autonomous SAR missions. the dotted line in the object depicts the virtual tilted plane used for pose estimation purposes. Points $\mathrm{p}_{0}$ to $\mathrm{p}_{3}$ are projected onto the image plane for IBVS purposes.

\subsubsection{Feature Extraction System}

\section{Shape and Color-Based Object Detector}

The design of this component takes into account the severe computational constraints related to the development of fully-autonomous missions, where a large number of processes should run in parallel inside the computer of the aerial robot. For this purpose, this component is based on lightweight computer vision algorithms, which use the prior information of the objects to detect, similar to the region proposal stage integrated into the helipad detection algorithm described in Section 2.3.

For addressing the aforementioned constraints, the functionalities implemented within this component are based on two main features: color and aspect ratio information. The former is based on heuristics extracted from the use case of IMAV 2016, in which the targets were defined as red and blue buckets or items. In order to segment the image based on color information, the HSV color space has been utilized, whose optimal ranges for each channel have been empirically derived. Aspect ratio information is mainly utilized for differentiating between identical objects in terms of 3D shape and color (e.g., a red bucket is defined as a cylinder of $0.25 \mathrm{~m}$ radius and $0.3 \mathrm{~m}$ height, while a red item consists of a cylinder of $0.1 \mathrm{~m}$ radius and $0.1 \mathrm{~m}$ height).

Using these functionalities, the main objective of the Shape and Color-Based Object Detector is to generate region proposals to the Model-Based Object Detector component and is also utilized as the main object detection algorithm when the image is captured using the bottom camera of the UAV. Furthermore, using the combination of aspect ratio and color information, this component provides an intra-class classification of the detected object (e.g., item $A$, bucket $A$, itemB, bucket $B$, where all these objects are cylinders and only 
differ in the color and aspect ratio as stated previously). Additionally, this component has been conceived for facilitating the rescue operation in SAR missions, which involve a prior pose estimation stage of the detected target. Since this component provides object proposals based on color information, the Region Of Interest (ROI) of the detected object in the image fits its contour accurately. This can benefit posterior pose estimation stages as compared to other object proposal algorithms based on predefined ROI sizes such as sliding window approaches, where the ROI can be slightly away from the object contour.

\section{Model-Based Object Detector}

Object detectors based on predefined knowledge of the object to be detected, such as color, shape, etc., can be very specific and prone to false positives. In order to provide a robust detection of the target, reducing its vulnerability to the environment conditions (e.g., lighting conditions), this component incorporates a supervised learning classifier for target/background classification.

The objective of this component is to detect the object of interest (target) within the image plane, by providing its corresponding ROI, and besides, it is responsible for the detection of the target in terms of its 3D location with respect to the frame of reference of the camera. For achieving the aforementioned capabilities, the Model-Based Object Detector mimics the design of the helipad detection algorithm described in Section 2.3. Thus, this component is based on four main modules: a region proposal module which uses the Shape and Color-Based Object Detector described previously, a feature extraction module using HOG features, a supervised learning classifier, and a pose estimation module. The main functionalities of these modules have been explained in Section 2.3. Additionally, in this component we also evaluate the performance of different CNN architectures in substitution of the second and third modules, since CNNs act as an unsupervised feature extractor in combination with a supervised learning classifier. The details of the Model-Based Object Detector and the results obtained in its evaluation are described in Appendix B.

\subsubsection{Motor System}

\section{Motion Controller}

The Motion Controller utilized in our system architecture (Pestana et al., 2014), is based on a cascaded control loop architecture where the external loops are based on a combination of PD controllers which act in position and the inner loops control the linear velocity of the UAV using a combination of PID controllers. This Motion Controller operates in combination with the attitude (i.e., roll, pitch, and yaw) and altitude control loops which are usually implemented inside the autopilot of the corresponding aerial robot.

Three main flight modes are available within the Motion Controller: trajectory, position or velocity, which can be switched during flight. In the experiments conducted for SAR missions, the proposed mission planning system is able to automatically switch from position to velocity control mode when the RL-IBVS controller is activated in order to perform the rescue operation for releasing the preloaded item. 
Some of the capabilities of the Motion Controller that make it suitable for our purposes include a native saturation of the linear velocity commands in all flight modes and the capability of tuning the control gains after performing system identification of a simple dynamic model. These functionalities allow a smooth navigation in cluttered environments, decreasing potential motion blur in the images captured onboard the UAV.

\section{IBVS Controller}

Two variants of IBVS controllers have been used during the SAR experiments presented in Section 6.7. Both controllers operate over the detection provided by the Shape and Color-Based Object Detector using the image provided by the UAV's bottom camera.

- Geometry-based IBVS controller (Sampedro et al., 2017): Given the current detection of the object and its desired location in the image plane, this controller uses the pinhole camera model to translate these errors in the image plane to errors in the position of the camera. Subsequently, the rigid transformation of the camera with respect to the UAV frame of reference (see Fig. 6.4 is used to compute the errors in the position of the UAV. These errors are used in conjunction with the UAV position estimations in world coordinates in order to obtain the references for the Motion Controller (in position control mode) in world coordinates.

Visual feedback is provided to the controller in the form of estimations of the location of the object of interest in the image plane. These estimations are computed by the Shape and Color-Based Object Detector component and delivered as ROIs. Given these ROIs, Eq. 6.6 considers a pinhole camera model to estimate position errors of the camera with respect to the object of interest. In this equation, $\Delta X, \Delta Y$ and $\Delta Z$ represent the position errors of the onboard bottom-looking camera with respect to the object of interest in meters, $\left(x_{r e f}, y_{r e f}\right)$ are the image coordinates of the desired positions of the center of the ROI, $(x, y)$ are the current ROI's $2 \mathrm{D}$ coordinates, $d_{r e f}$ is the desired hovering distance above the object of interest, $f x, f y$ are the camera's focal lengths in pixels, $O_{a}$ is the object area, measured in squared meters, and $A_{i m}$ is the area of the ROI (width $\times$ height).

$$
\left[\begin{array}{c}
\Delta X \\
\Delta Y \\
\Delta Z
\end{array}\right]=\left[\begin{array}{c}
\frac{\left(x_{r e f}-x\right) d_{r e f}}{f_{x}} \\
\frac{\left(y_{r e f}-y\right) d_{r e f}}{f_{y}} \\
d_{r e f}-\sqrt{\frac{f_{x} f_{y} O_{a}}{A_{i m}}}
\end{array}\right]
$$

The desired positions of the center of the ROI are obtained empirically. These positions correspond to the $2 \mathrm{D}$ coordinates of the ROI's center when the UAV is located at the desired position with respect to the object of interest.

As a safety measure, if estimated position errors are greater than $2 \mathrm{~m}$ in any direction no updates in the position reference are given to the Motion Controller. The same criterion is applied if the distance between the last computed position reference and the current one is greater than $2 \mathrm{~m}$ in any direction. 
Additionally, a buffer accumulating the last $n$ position errors has been implemented and can be used as an indicator of the positioning stability in order to automatically trigger specific actions involving the interaction with the object of interest (e.g., pick up, release).

- RL-IBVS controller (Sampedro et al., 2018b): This controller has been used in the last SAR experiment presented in Section 6.7, and is described in detail in Chapter 4 .

\subsection{Experiments and Results}

The following sections describe the experiments conducted in order to evaluate the capabilities of the proposed system and validate its integration into the Aerostack architecture. For this purpose, we first present in Section 6.6 the results obtained when using the proposed mission planning system in the coordination of a swarm of UAVs for the execution of high-level missions (i.e., find target and explore). Subsequently, Section 6.7 describes the results obtained in the application of the proposed mission planning system for building a fully-autonomous UAV in order to the execute SAR missions. Several videos of the reported experiments and results can be found in:

- Using swarms: https://youtu.be/2EHbb3y3U08

- Using a fully-autonomous UAV for SAR missions:

- https://youtu.be/y9Bj3deT-Kw

○ https://vimeo.com/235929544

\subsection{Experimental Results using Swarms of UAVs}

This section presents the simulation and real flight experiments that have been conducted in order to evaluate the proposed mission planning system using swarms of UAVs for performing different high-level missions (see Table 6.2). To this aim, we first describe the experimental setup used for the simulation and real flight experiments. Subsequently, we present the simulation experiments in Section 6.6.2, and the real flight experiments in Section 6.6.3.

Table 6.2: Experiment set designed for real and simulation flights using swarms of UAVs

\begin{tabular}{ccccc}
\hline Mission type & Map Size & Type of flight & \# UAVs & \# Obstacles \\
\hline Find Target & $9 \mathrm{~m} \times 10 \mathrm{~m}$ & Real \& Sim. & 2 & 6 \\
Find Target & $20 \mathrm{~m} \times 20 \mathrm{~m}$ & Simulation & 4 & 11 \\
Explore & $20 \mathrm{~m} \times 30 \mathrm{~m}$ & Simulation & 6 & 12 \\
\hline
\end{tabular}




\subsubsection{Experimental Setup}

In both simulation and real-flight experiments, several computers have been used for running the required components in the Aerostack architecture for each agent in the swarm. The software used in the proposed architecture is built in $\mathrm{C}++$, under the standard $\mathrm{C}++11$, using the Robot Operating System (ROS) (Quigley et al., 2009) as the communication middleware. Simulation flight experiments have been conducted using the simulator integrated into the Aerostack architecture, and using ROS Rviz for visualization purposes. Regarding the real flight experiments, a flying area of $9 \mathrm{~m} \times 10 \mathrm{~m}$ has been used. Using this scenario, several flights have been conducted using a swarm of two UAVs. The UAV platform selected for real flight experiments is the Parrot ArDrone 2.0, which provides a low-cost and robust platform for testing purposes. For localization and mapping, ArUco fiducial markers (Garrido-Jurado et al., 2014) have been utilized. These markers provide a robust and real-time pose estimation, and are mainly used in the proposed experiments for localization, obstacle avoidance, and for simulating the target to be detected during the find target missions.

\subsubsection{Simulation Experiments}

Using the experimental setup described in Section 6.6.1 for the simulation experiments, three simulation scenarios (see Fig. 6.5) have been designed, varying the number of agents in the swarm, the number and location of the obstacles in the scenario, and the dimensions of the latter. Two of these scenarios (see Fig. 6.5a and 6.5b have been conceived for testing the capabilities of the proposed mission planning system for conducting find target missions. The scenario presented in Fig. 6.5c simulates a $20 \mathrm{~m} \times 30 \mathrm{~m}$ area where a swarm of $6 \mathrm{UAVs}$ is required to explore the scenario in a coordinated way.

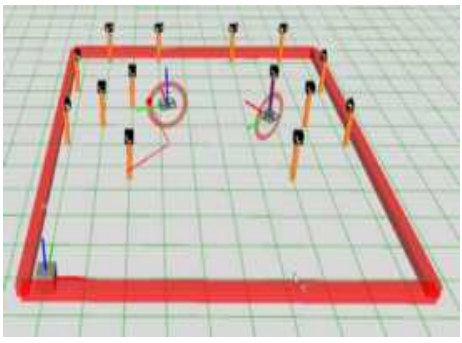

(a)

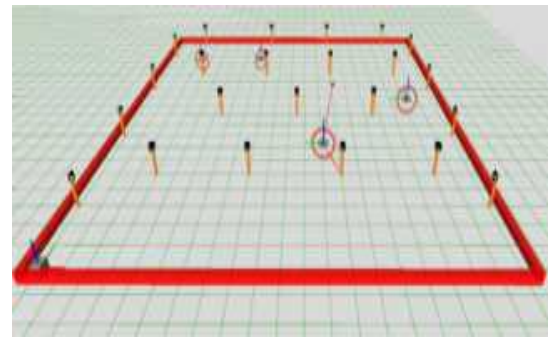

(b)

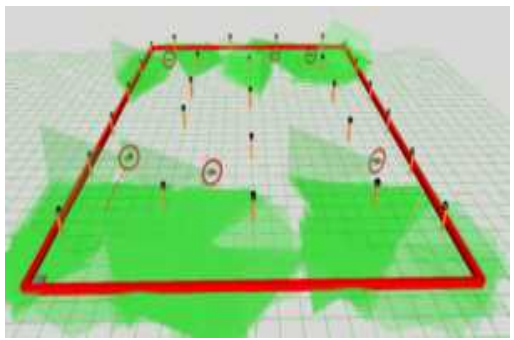

(c)

Figure 6.5: Simulation scenarios designed using ROS Rviz for evaluating the proposed mission planning system while managing swarms of UAVs. (a) Simulation scenario for the find target mission in a $9 \mathrm{~m} \times 10 \mathrm{~m}$ map, using 2 UAVs. (b) Simulation scenario for the find target mission in a $20 \mathrm{~m} \times 20 \mathrm{~m}$ map, using 4 UAVs. (c) Simulation scenario for the explore mission in a 20 $\mathrm{m} \times 30 \mathrm{~m}$ map, using 6 UAVs. Green color simulates the explored area that is being covered by the swarm.

In order to obtain a better understanding of the proposed experiments, in the following paragraphs we describe the main characteristics of the find target and explore missions: 
- Find target: In this mission, the inputs to the GMP are the dimensions of the area to be explored, the number of mission points, and the high-level command specifying the type of mission to be conducted. The objective of the mission is to explore the unknown area (i.e., the position of the obstacles are not known) in order to find a target represented by an ArUco visual marker, whose location is also unknown. Once any agent in the swarm detects the target, its corresponding AMP sends the event of target found to the GMP, which re-plans the mission in real-time according to the following procedure:

- The agent who has found the target performs a flip maneuver to highlight the event for the human operator. Subsequently, the agent is commanded to inspect the target. Finally, once the inspection has been completed, the agent is sent to its initial take-off point, performing a landing maneuver when the initial point is reached.

- The remaining agents in the swarm are sent to their respective initial take-off points, performing a landing maneuver when the initial point is reached.

The mission of find target is finished when the target is found and all the agents in the swarm have returned to their respective initial take-off points, or when the object is not found and all the mission points have been reached.

- Explore: In this type of mission, the inputs to the GMP are the same as in the find target mission, with the difference that in this case the GMP receives a high-level command of explore. The objective of this mission is to explore the designated area in a coordinated way. In the simulation experiments conducted in this section, we simulate the Field Of View (FOV) of the UAV by considering the FOV of the camera mounted onboard the Parrot ArDrone 2.0.

In both find target and explore missions, the behavior conducted by each agent during the execution of the main tasks is as follows:

- Move To Point. The agents move in position control mode to the commanded 3D point that is established in the corresponding task.

- Turn In Yaw at different angular steps to complete a $360^{\circ}$ turn, while maintaining the current 3D position. This behavior is performed for exploring the surroundings of the mission point reached by the UAV.

The results obtained after the execution of the simulation experiments are shown in Fig. 6.6. In this figure, Fig 6.6a, 6.6b, and 6.6c show examples of the Voronoi regions created after sampling the Mission Zone, using the number of mission points specified in the corresponding mission. The centroids of the Voronoi regions (see "X" white marks) after the sampling stage, are used by the GMP as mission points in the corresponding mission.

The results obtained in the execution of the find target mission are shown in Fig. 6.6d and Fig. 6.6e. In these figures, it can be observed how the mission points are distributed 


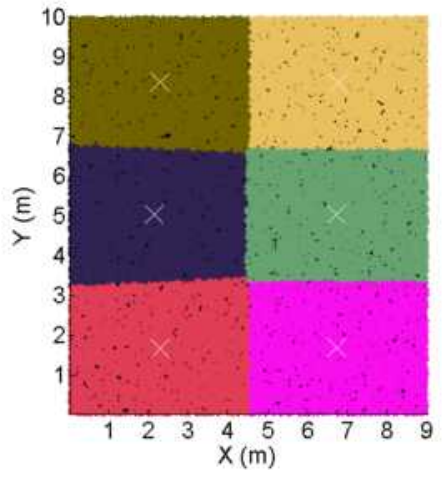

(a)

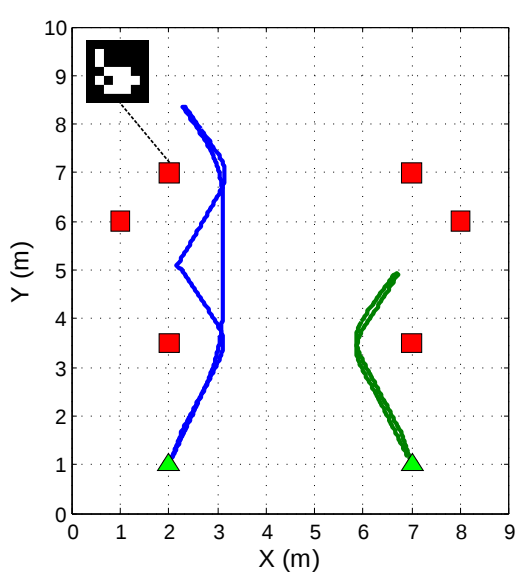

(d)

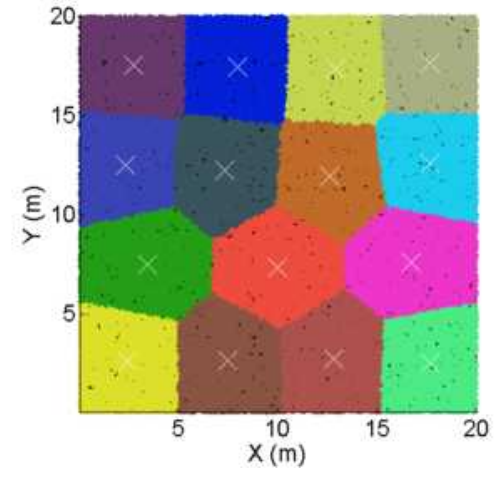

(b)

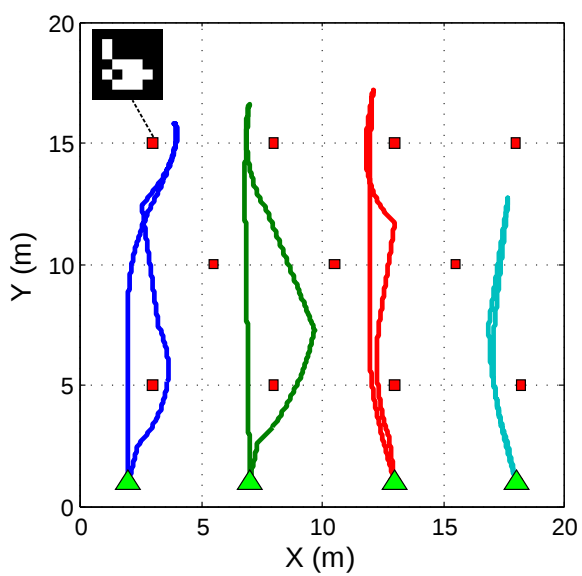

(e)

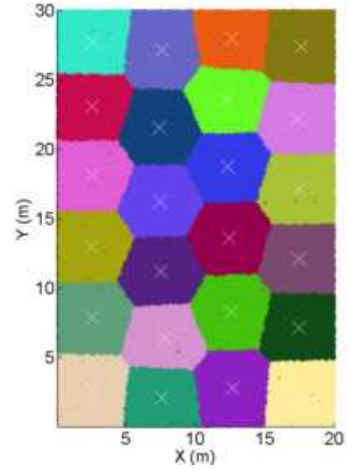

(c)

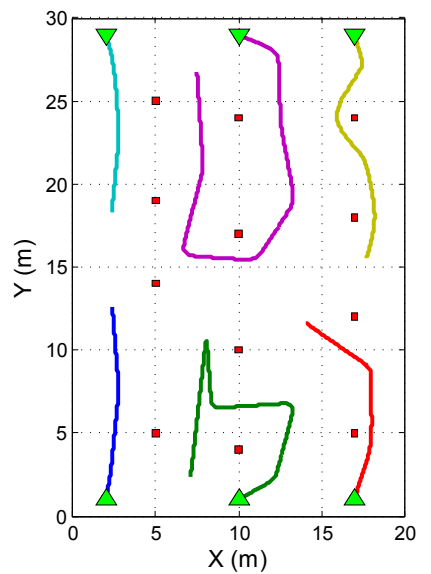

(f)

Figure 6.6: Simulation results obtained in the different experiments conducted using swarms of UAVs. (a), (b), (c) Voronoi regions generated by sampling the Mission Zone. "X" white marks correspond to the centroids of the clusters that will be used as mission points. (d), (e), (f) Trajectories generated by the different agents in the swarm while completing their respective missions. Green triangles depict the take-off point of the different UAVs in the swarm. Red squares represent the obstacles. Targets are simulated using ArUco visual markers.

through the agents in the swarm, whose trajectories are planned for visiting each of the commanded mission points, avoiding the obstacles, and searching for the target. In both experiments, the target was found by the UAV agent with blue trajectory. This event triggered the inspection stage conducted by the UAV who found the target, and the maneuver for returning to the take-off point conducted by the remaining agents in the swarm. The trajectories generated by the UAVs in Fig. 6.6d are of particular interest since it can be clearly observed the re-planned mission performed by the GMP. The mission of the agent with blue trajectory is started approximately $2 \mathrm{~s}$ before the mission of the agent with the green trajectory. This produced that the agent with blue trajectory encountered the target at first place, and thus, the second agent is commanded to the initial take-off point in an early stage of its mission. Similar behavior can be noticed in Fig. 6.6e while analyzing the trajectories generated by the first and fourth UAVs in the swarm (see blue 
and cyan trajectories in Fig. 6.6e).

The results obtained after the execution of the explore mission are shown in Fig. 6.6f. As in the find target mission, in Fig. 6.6f it can be observed the distribution of the mission points performed by the GMP in order to optimize the resources of the swarm. Once the mission points are distributed through the agents, the AMP schedules the Move To Point tasks (among the other tasks assigned in the mission) in order to explore all the required points. As shown in Fig. 6.6f the UAVs whose take-off point is located at coordinate $x: 10 \mathrm{~m}$, are assigned most of the mission points (5-6 points per agent), while the UAVs with take-off points located at the lateral parts of the scenario are assigned 3-4 points to explore.

\subsubsection{Real-flight Experiments}

In this section, we present the real flight experiment analogous to the simulation flight presented in Fig. 6.5a and Fig. 6.6d. Using the experimental setup described in Section 6.6.1, we build a real flight scenario of $9 \mathrm{~m} \times 10 \mathrm{~m}$ (see Fig. 6.7a), where the obstacles and target are represented by ArUco visual markers. As in the case of the analogous simulation experiment, this experiment simulates a find target mission, where the objective is to find the target located at coordinates $(x: 0 \mathrm{~m}, y: 8 \mathrm{~m}, z: 1.3 \mathrm{~m})$.

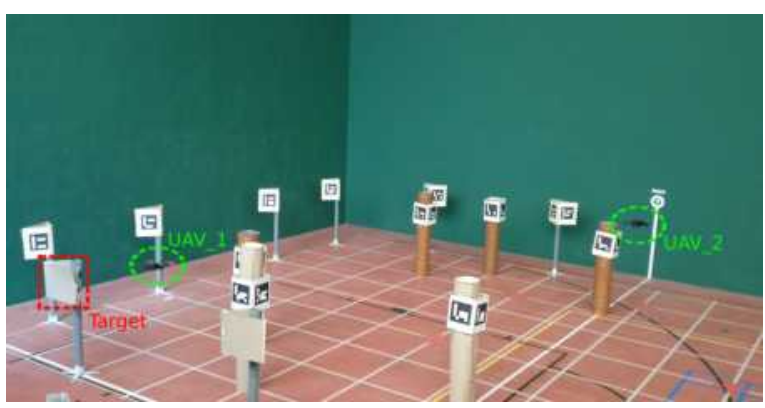

(a)

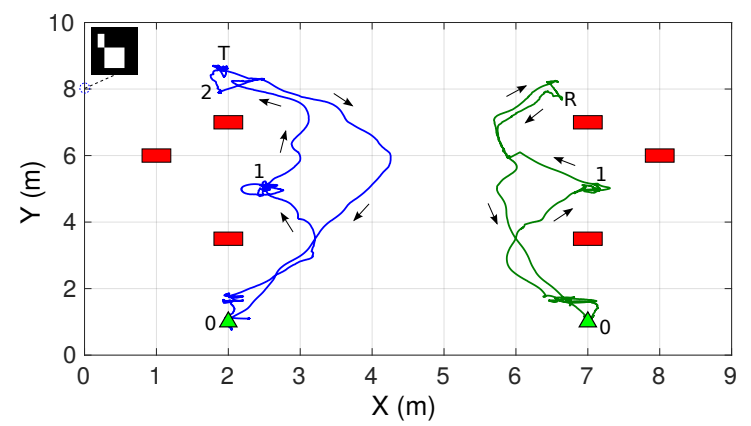

(b)

Figure 6.7: Real flight experiment using two UAVs for a find target mission in a $9 \mathrm{~m} \times 10 \mathrm{~m}$ map. (a) Real flight scenario. (b) Trajectories generated by the two UAVs during the mission. Green triangle depicts the take-off point. Red objects represent the obstacles.

The trajectories generated by the UAVs during the execution of the mission are shown in Fig. 6.7b, which in general reveal the good reproducibility of the experiment in realflight conditions. In this figure, it is highlighted the point "T" where the agent with blue trajectory starts inspecting the target, once it is detected. At this point, the trajectory of the UAV has some displacements in the $x$ and $y$ directions since the UAV performs a flip maneuver once the target is detected to highlight the event to the human operator. On the other hand, the point " $\mathrm{R}$ " in the trajectory of the second agent is highlighted since at this point the agent receives the new mission of returning to the initial take-off point. Overall, it can be observed a similar behavior as obtained in the analogous simulation experiment, validating the proposed mission planning system. 


\subsection{Experimental Results in SAR missions}

This section presents the simulation and real flight experiments that have been conducted in order to evaluate the proposed mission planning system while performing SAR missions. More concretely, in this section we again validate the re-planning capabilities showed in Section 6.6, and evaluate the new re-planning capabilities integrated into the system in order to address the rescue operation in SAR missions (i.e., interacting with the target by means of releasing a preloaded item, following the use case of IMAV 2016). For this purpose, we first describe in Section 6.7.1 the experimental setup used for the simulation and real flight experiments. Subsequently, we present the simulation experiments and results in Section 6.7.2, and the real flight experiments and results in Section 6.7.3.

\subsubsection{Experimental Setup}

In this section, the proposed experimental setup for simulation and real flights is described. All the developed software components have been implemented within the Aerostack architecture using $\mathrm{C}++$, under the standard $\mathrm{C}++11$, and using ROS as the communication middleware between the different components. Ubuntu 14.04 LTS operating system has been utilized for running the different processes in both simulation and real flights.

\section{Simulation Flights}

A simulation environment has been created using the Gazebo simulator in combination with PX4 Software-In-The-Loop ${ }^{5}$ (SITL). The designed simulation using Gazebo and PX4 SITL provides a realistic simulation environment with appropriate communication infrastructure (using ROS), sensors, and dynamics for controlling the aerial robot. In the case of the PX4 SITL the default multirotor available is the IRIS from 3DRobotics, which is the one used through the simulation flights presented in this section.

Following the guidelines proposed in the IMAV 2016 competition rules, and with the purpose of obtaining a complete validation of the proposed system, several simulation scenarios of dimensions $13 \mathrm{~m} \times 13 \mathrm{~m}$ (see Fig. 6.9a and 6.9c) have been designed, incorporating variability between each other in terms of layout, location of obstacles, position of the targets (i.e., buckets), etc.

\section{Real Flights}

A real indoor environment with dimensions $9 \mathrm{~m} \times 11 \mathrm{~m}$ has been designed, in which the Mission Zone consists of a rectangular area of $9 \mathrm{~m} \times 6 \mathrm{~m}$. With the aim of testing the proposed system in a similar scenario to the real one designed by the IMAV 2016 competition, a replica of the main façade has been built, in which the dimensions of the possible entries (i.e., door and window), as well as the distance between them, are exactly the same as those stated in the IMAV 2016 rules.

\footnotetext{
${ }^{5}$ https://github.com/PX4/sitl_gazebo
} 
Using the Mission Zone presented in Fig. 6.10, several real flights have been conducted in order to perform a thorough validation of the proposed system in SAR missions conducted in indoor scenarios. Inside the Mission Zone, several layouts have been designed, modifying the number and dimensions of the rooms and the location of obstacles (i.e., columns, tables, chairs, etc.) and objects to be found.

As the aerial robotic platform, we use a custom aerial quadrotor shown in Fig. 6.8. The proposed UAV has relatively small dimensions $(62 \mathrm{~cm} \times 62 \mathrm{~cm} \times 40 \mathrm{~cm}$, including propellers of $28 \mathrm{~cm}$ ), and has been designed and built with a total takeoff weight of $3.2 \mathrm{~kg}$, a maximum payload capacity of $1 \mathrm{~kg}$, and an approximate flight time of 12 min. The onboard computer consists of an Intel NUC6i5SYK featuring a $2.9 \mathrm{GHz}$ Intel Core i5-6260U CPU. The avionics of the UAV are managed by a Pixhawk (Meier et al., 2011) autopilot, which integrates an Inertial Measurement Unit (IMU), a barometer and a magnetometer. The exteroceptive sensors mounted onboard consist of a Hokuyo laser rangefinder UTM-30LX with a horizontal field of view of $270^{\circ}$ and an angular resolution of $0.25^{\circ}$ with a maximum range of $30 \mathrm{~m}$, an Intel Realsense R200 camera with an RGB image size of $640 \times 480$ pixels, a standard RGB $180^{\circ}$ fisheye-lens bottom-looking camera with an image size of $640 \times 480$ pixels, and a Lightware SF10/A altimeter with a maximum range of $25 \mathrm{~m}$ (see Fig. 6.8a). The communication between the autopilot, proprioceptive, exteroceptive sensors and the onboard computer is performed over USB connections. In addition, appropriate electronic components and holding devices have been designed and integrated for object interaction tasks. For this purpose, two small curved hooks have been integrated into the UAV framework (see Fig. 6.8b). These hooks are controlled by two servo motors which are actuated via an Arduino board when a signal of target locked is commanded in order to release the items preloaded onboard the UAV.

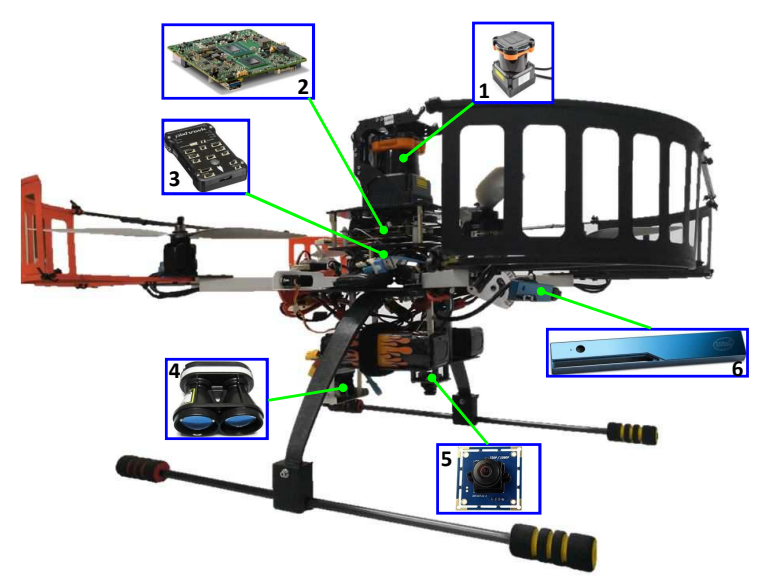

(a)

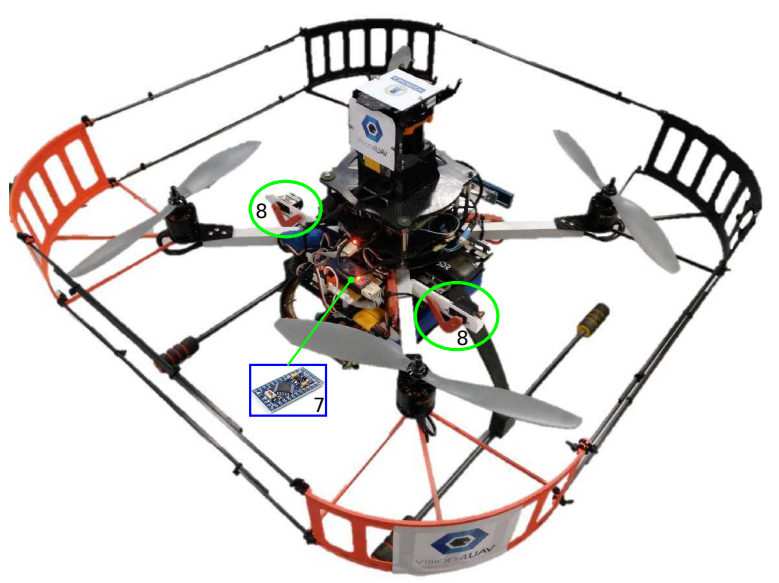

(b)

Figure 6.8: Proposed aerial robot used for SAR missions, showing 1) Hokuyo laser rangefinder UTM-30LX. 2) Intel NUC6i5SYK computer. 3) Pixhawk autopilot. 4) Lightware SF10/A altimeter. 5) Fisheye bottom camera. 6) Intel realsense R200 camera. 7) Arduino pro-mini. 8) Designed hooks for holding items. 


\subsubsection{Simulation Experiments}

This section describes the simulation experiments that have been designed in order to evaluate the coordination between the proposed mission planning system and the remaining components of the whole aerial robotic architecture to accomplish SAR missions in cluttered indoor scenarios. For the evaluation of the entire system, several simulation scenarios using the Gazebo simulator (see Fig. 6.9a and 6.9c have been designed based on the scenario proposed for the IMAV 2016 competition. Inspired by the use case of the IMAV 2016, different layouts of indoor rooms have been created. In this experiment, the objective of the UAV is to explore the a priori unknown indoor scenario searching for predefined targets (red and blue buckets). Once a target has been detected, the UAV has to interact with the former to release an item inside of it for rescue purposes (e.g., simulating the release of a medical kit). Once the interaction is finished, the UAV continues the previous exploration mission in order to locate more possible targets. As shown in Fig. $6.9 \mathrm{~b}$ and $6.9 \mathrm{~d}$, the UAV is able to explore the entire indoor scenario, re-adapting the exploration mission when a target is detected in order to perform the release maneuver (see points $R_{1}$ and $R_{2}$ in Fig. 6.9b, and $R_{1}$ in Fig. 6.9d). As shown in Fig. 6.9b after reaching mission points 2 and 3 , the UAV finds the corresponding targets (red and blue buckets, respectively). This event (target found) causes the GMP to introduce a new rescue mission in the active list of missions that it handles, thus queuing the previous exploration mission that was being executed. After finishing the rescue operation, the GMP recovers the exploration mission in the corresponding state. This can be observed in Fig. 6.9b after the $R_{1}$ and $R_{2}$ points, when the UAV continues its exploration mission moving towards point 3 and 4, respectively. A similar behavior can be noticed in Fig. 6.9d when the UAV finds the target located at point $R_{1}$. Another remarkable behavior that can be noticed in Fig. 6.9d is the safety point (see point 2s in Fig. 6.9d) generated by the AMP in order to avoid the mission point number 2 which is within the boundaries of an obstacle, taking into account the inflation radius of the obstacles defined in the Path Planner component. This behavior validates the mission point reallocation capabilities of the proposed AMP.

\subsubsection{Real-flight Experiments}

In this section, three real-flight SAR experiments have been designed for evaluating the whole system proposed in this work. In the designed experiments, the UAV is required to autonomously explore a cluttered indoor scenario with two possible entries: a $1.2 \mathrm{~m}$ $\times 2.0 \mathrm{~m}$ door or a $1 \mathrm{~m} \times 1 \mathrm{~m}$ window. The indoor scenario is composed of several obstacles, mainly tables, chairs and columns, whose location is completely unknown (see Fig. 6.10). Based on the use case of the IMAV 2016, the targets are represented by two colored buckets. The objective of the UAV is to autonomously explore the indoor scenario, localize the targets, and interact with them by means of performing a release maneuver of the corresponding item. The items, both red and blue, are preloaded before take-off and hold in the hooks of the UAV. Once the interaction with the targets is finished, the UAV has to return to the initial takeoff point. As shown in Fig. 6.10, three scenarios of incremental degree of complexity have been designed. The first scenario 


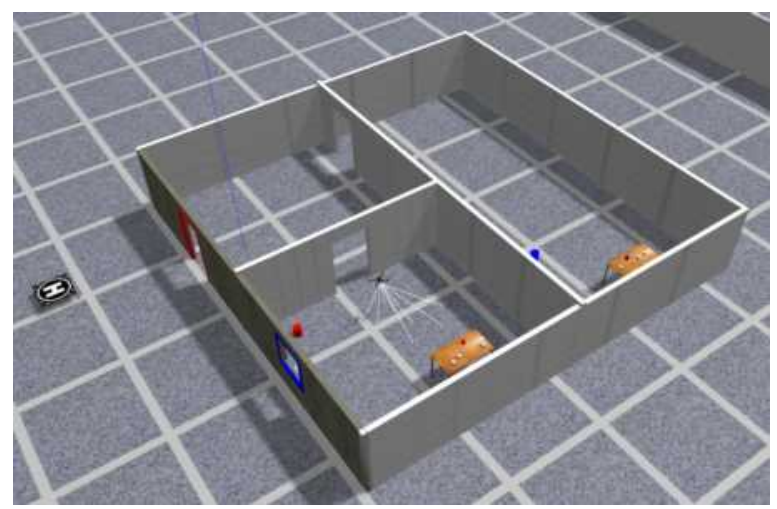

(a)

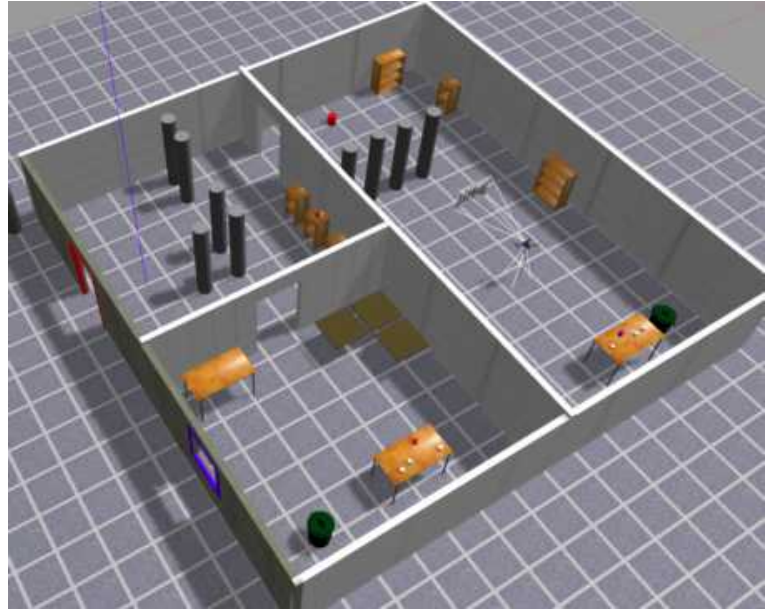

(c)

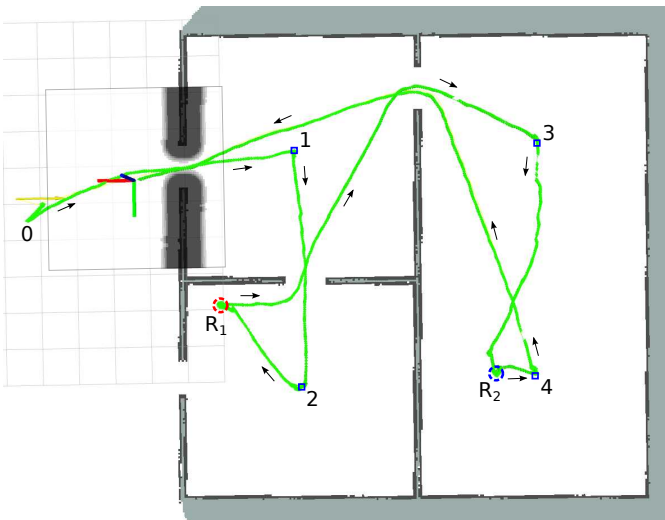

(b)

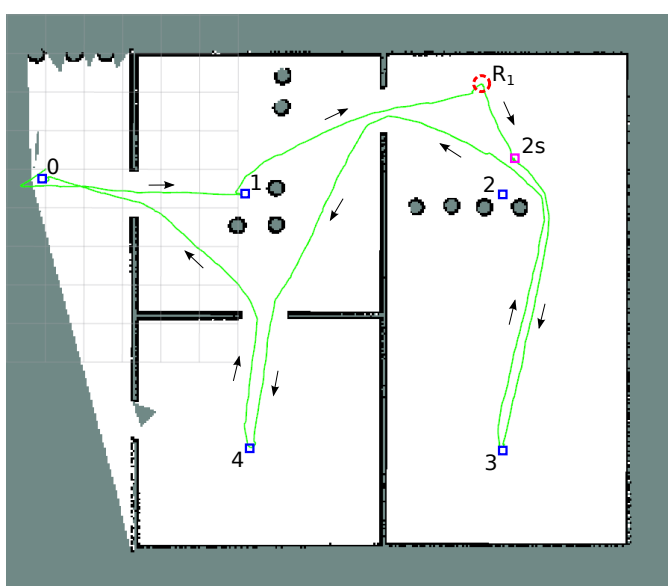

(d)

Figure 6.9: Simulation flight experiments for SAR missions. (a), (c) Gazebo environments designed for reproducing the IMAV 2016 competition layout. (b), (d) 2D occupancy grid maps and trajectories generated by the UAV (green color). The colored squares depict the mission points automatically generated by the GMP, while the colored circles represent the Buckets.

shown in Fig. 6.10a consists of a rectangular area with two main rooms and no obstacles. This scenario was primarily conceived for validating the rescue maneuver, and also for validating the landing maneuver on top of a moving platform (see Fig. 6.11b). The second scenario (see Fig. 6.10b) consists of the same rectangular area used in the first scenario, where several obstacles (e.g., tables) are placed at random positions, and the two buckets are located close to each other and close to a dividing wall between the two, with a double purpose of validating the simultaneous detection of several objects, and the management of these detections in terms of planning (i.e., the concatenation of both rescue missions). On the other hand, the location of the buckets close to a wall aims at validating the precision of the Path Planner and Visual Servoing components in the accomplishment of the rescue operation. Finally, the third scenario (see Fig. 6.10c) increases the difficulty in terms of number and position of the obstacles within the scenario. Besides, in this scenario 
the blue bucket is located on top of a table and the red one is located on the floor, with the purpose of validating the rescue maneuvers in very different conditions. This validation involves the correct performance of the perception components which should provide a proper estimation of the position of the targets, the state estimation component in order to filter possible obstacles that can be below the UAV while navigating and performing the rescue maneuver, the visual servoing component for a correct release operation, and the Mission Planning system for automatically switching between perception devices (i.e., switching perception between front and bottom camera and vice versa when the bucket has been detected and the item has been released, respectively), among others. Moreover, as in the other real-flight scenarios and in the simulation experiments, the validation of the capabilities for concatenating different missions and the ability to switch between them is one of the main objectives of this experiment.

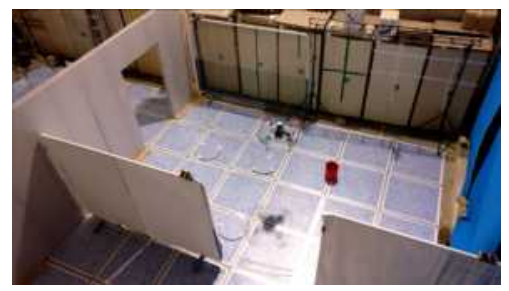

(a)

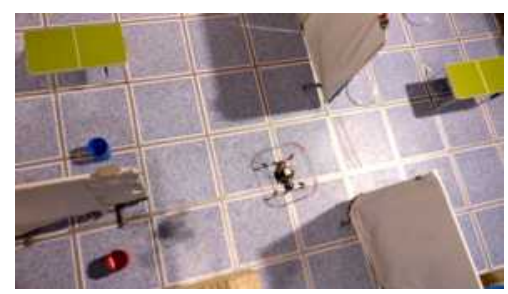

(b)

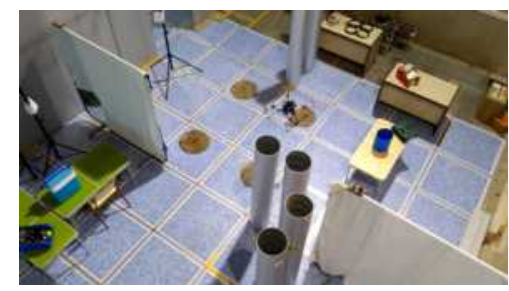

(c)

Figure 6.10: Real-flight scenarios designed for evaluating the proposed system in SAR missions.

The results obtained during the execution of the different SAR missions in the three indoor scenarios described above are presented in Fig. 6.11. The results obtained for the SAR mission in the first scenario are shown in Fig. 6.11a and 6.11b. These results reveal that the proposed mission planning system is able to correctly manage the whole mission where the UAV is capable of autonomously explore the unknown scenario searching for the two buckets, detecting them, performing the corresponding release operations, and executing the autonomous landing maneuver on a moving platform at the final commanded mission point. The results presented in Fig. 6.11c and 6.11d for the second SAR mission, reveal the complex situations that the proposed mission planning system is able to handle. In this experiment, the role of the Perception Manager component is of utmost importance since it handles the simultaneous detection of several objects. For this purpose, this component has an internal buffer for storing the pose of the objects being detected over time. This buffer is utilized by a filter in which the poses away from the average pose are removed in an iterative process. Once the number of correct detections for the corresponding target reaches a predefined threshold, the event of target found is sent to the GMP which is capable of handling simultaneous target found events. In the specific case of the experiment shown in Fig. 6.11d, the first target found event corresponds to bucket $\mathrm{A}$, and the analogous event for bucket $\mathrm{B}$ is queued in the list of missions. Thus, the GMP gives priority to perform the rescue maneuver on this bucket. For this purpose, the new rescue mission starts by commanding the UAV to the mission point generated by the estimated position of bucket A in the map. Once the UAV reaches the mission point, in which the corresponding bucket is located, the next task of performing visual servoing is 
scheduled by the AMP, based on the visual reference given by the detection on the image captured by the bottom camera. For obtaining an accurate release maneuver, the Visual Servoing component commands the UAV at $0.7 \mathrm{~m}$ height, which can be well appreciated in Fig. 6.11d at position [x: $8.5 \mathrm{~m}, y: 9.5 \mathrm{~m}]$. Once the rescue mission of bucket $\mathrm{A}$ is finished, the GMP immediately recovers the rescue operation for the remaining target (i.e., bucket B), and proceeds analogously as in the rescue mission of the first target.

Finally, Fig. 6.11 shows the 2D map generated (see Fig. 6.11e) and the 3D trajectory followed by the UAV (see Fig. 6.11f) when the SAR mission is completed in the third scenario. As can be observed in Fig. 6.11e the system is capable of adapting the current mission when the first target (blue bucket) is detected while going from mission point 1 to 2 . For this purpose, the mission planning system acts by queuing up the current exploration mission and adopting the rescue operation of the detected target as the current mission to accomplish. Once the UAV finishes the rescue operation on the first target, the previous exploration mission is recovered in its corresponding state. The same behavior can be noticed when the UAV is commanded from mission point 2 to 3 and the second target (red bucket) is detected. In this SAR mission, the main difference as compared to the missions executed for the first and second scenarios, is that the object detection tasks are performed using the Model-Based Object Detector component (see Section 6.4.4), and the Visual Servoing component in Sampedro et al., 2017 is replaced by the RL-IBVS algorithm presented in Chapter 4 (Sampedro et al., 2018b).

\subsection{Discussion}

In this chapter, a complete mission planning architecture for managing multi-robot systems has been presented. A thorough validation of the proposed system has been carried out in experiments with swarms of aerial robots, and in Search and Rescue missions using a fully-autonomous UAV.

Overall, the results presented throughout this chapter reveal the versatility of the proposed mission planning system for accomplishing high-level missions (e.g., SAR) which involve the management of a wide range of events that can occur during the execution of the mission (e.g., a target has been detected and a new mission for its inspection or rescue should be generated). The design strategies integrated into the GMP, such as the dynamic list of missions, allow the concatenation of several missions in response of different events. Furthermore, the capabilities integrated into the AMP, such as the dynamic mission point reallocation (see point 2s in Fig. 6.9d), provide a higher level of flexibility to the system, giving at the same time an additional safety level.

The results obtained using swarms of UAVs are illustrated in Fig. 6.6 and 6.7 for simulation and real-flight experiments, respectively. In these experiments, the sampling and mission point distribution capabilities implemented into the GMP have proven to provide an effective solution for managing complex high-level missions using a multi-robot system. More concretely, the proposed algorithm for sampling a Mission Zone based on a $k$-means clustering method provides an efficient solution in order to optimize the distribution of mission points within the area to be explored (see Fig. 6.6a, 6.6b, and 6.6c). Besides, the configuration of the proposed algorithm allows adapting the sampling stage to 


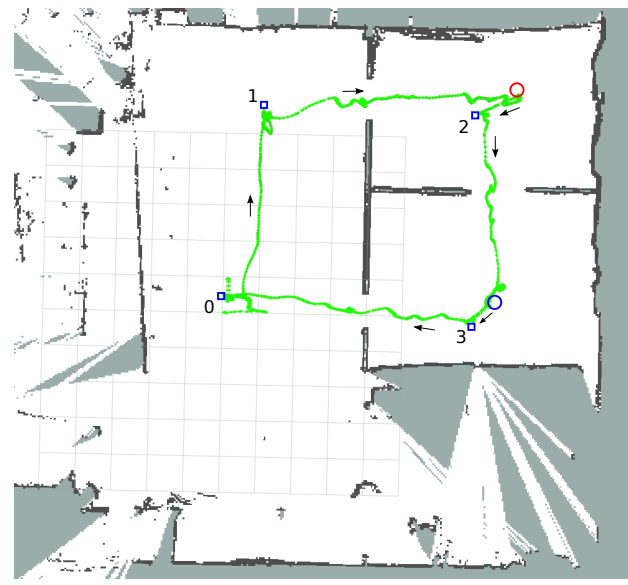

(a)

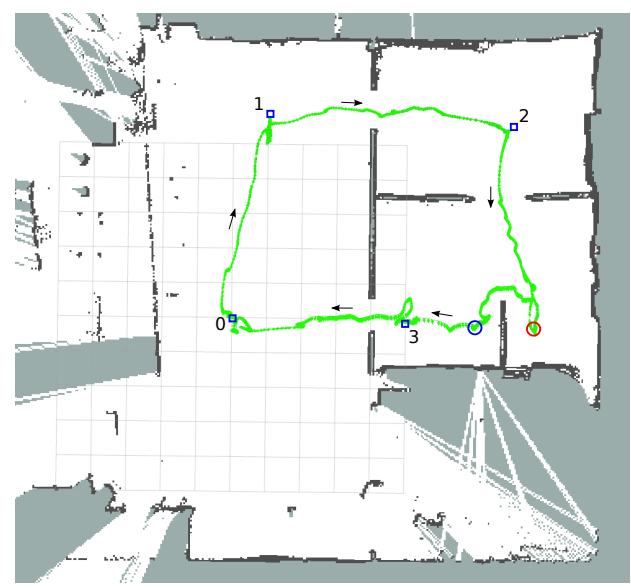

(c)

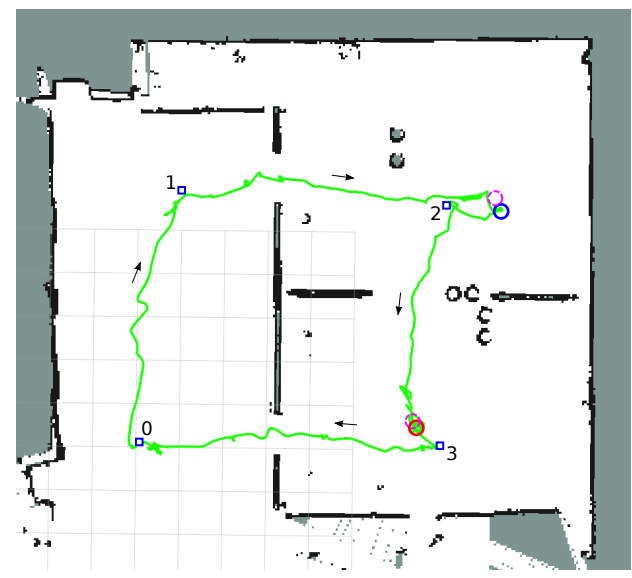

(e)

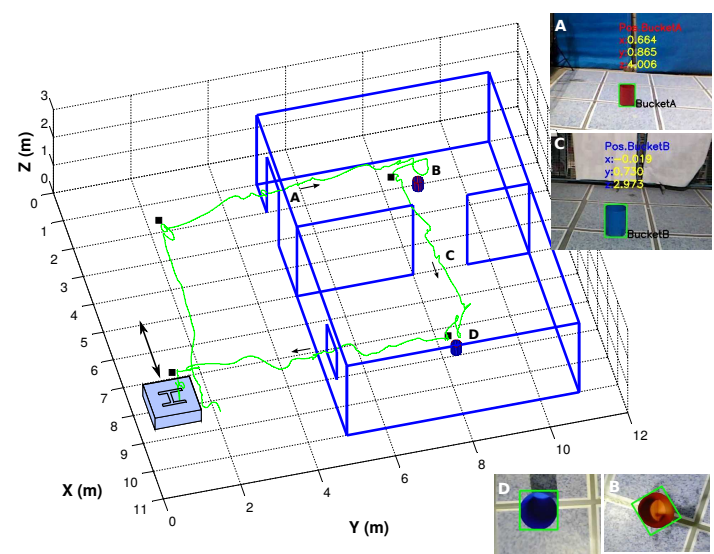

(b)

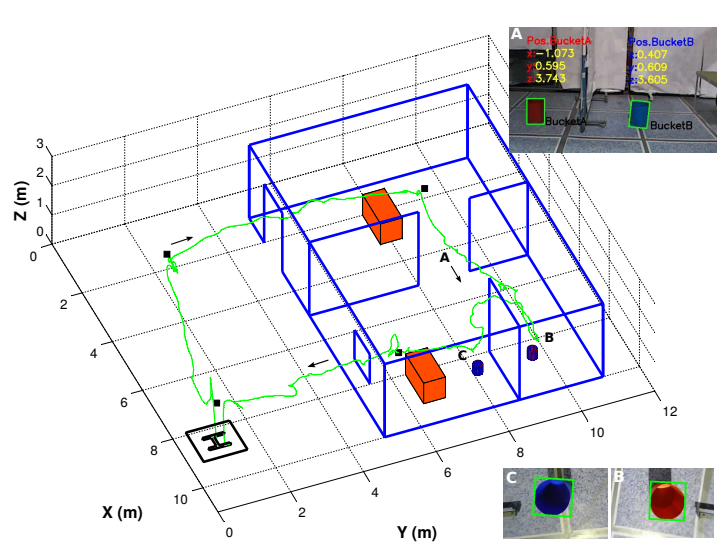

(d)

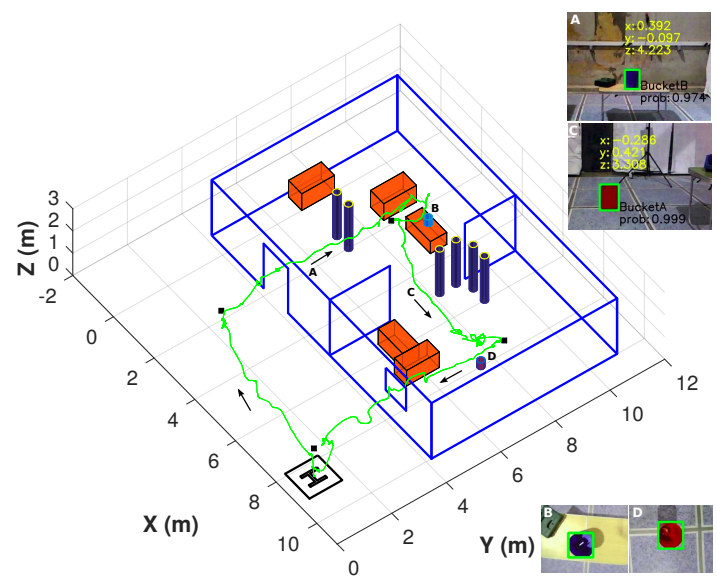

(f)

Figure 6.11: Real flight experiments conducted for SAR missions in three different indoor scenarios. (a), (c), (e) 2D occupancy grid map and trajectory generated by the UAV (green color). (b), (d), (f) 3D view of the trajectory performed by the UAV (green color). The Mission Zone outline is shown in blue color. Obstacles are represented by orange cuboids and the Buckets by their corresponding cylinder. The squares depict the commanded mission points. Images represent the object detection at the specific point of the trajectory. 
the specifications of the sensors used for each aerial robot to ensure a complete exploration of the Mission Zone (e.g., increasing the number of mission points when the FOV of the sensor is limited). The mission point distribution algorithm implemented into the GMP acts as a soft version of the Travelling Salesman Problem (TSP) and allows a fast nearto-optimal solution for the exploration of a sequence of mission points performed by each UAV (see Fig. 6.6f). The proposed mission point distribution algorithm can easily be adapted to the exact TSP variant, which will be considered for future works. In addition, the adaptation of the proposed distribution algorithm to run every time a mission point is reached will also be considered for future works. This improvement can provide an optimal solution in terms of energy resources, being of particular interest in big indoor scenarios explored using only one UAV. The results shown in Fig. 6.6d, 6.6e, and 6.6f reveal the outstanding capabilities of the proposed mission planning system for managing the find target and explore missions, which are accomplished optimizing the resources of the swarm as can be noticed in Fig. 6.6d and Fig. 6.6e. In these figures, it can be observed the shortest distance covered by the second (see green trajectory in Fig. 6.6d) and fourth (see cyan trajectory in Fig. 6.6e) agents due to the mission re-planning capabilities of the proposed system which sends the remaining agents to the initial take-off points in order to save resources while the agent that has detected the target starts its inspection. It should be noted that, for the accomplishment of similar missions involving targets of bigger dimensions (e.g., inspecting a whole building), the behavior of the swarm can be easily adapted using the proposed planning system in order to perform a coordinated inspection mission of the detected target.

A similar analysis can be obtained for the real-flight experiment depicted in Fig. 6.7. In this case, it should be emphasized the flexibility of the proposed mission planning system exhibited in the behavior of the first agent in the swarm (see the blue trajectory in Fig. 6.7b). In this case, the mission planning system allows obtaining a complex behavior of this agent, which can be summarized in the following sequence: exploration-detectionsignalization-inspection-return to the initial point. That is, the first agent conducts the main exploration mission which is stopped once this agent detects the target (see point 2 in Fig. 6.7b). At this point, a new mission is generated by the GMP consisting of first doing a flip maneuver (in order to highlight to the human operator the event of target found), and then the UAV is commanded to inspect the target (see point "T" in Fig. 6.7b). Finally, the UAV is sent to the initial take-off point once the inspection process is finished.

Regarding the results obtained for SAR missions, Fig. 6.9 illustrates the trajectories followed by the proposed fully-autonomous system while executing the designed SAR missions in two Gazebo simulation scenarios. Similarly, Fig. 6.11 depicts the trajectories generated while executing the proposed SAR missions in three real-flight indoor scenarios. The results shown in Fig. 6.9 further validate the versatility provided by the proposed planning system. In these experiments, it can be observed the outstanding performance of the list of missions integrated into the GMP, which allows the accomplishment of the rescue operations of the detected targets (see points $R_{1}$ and $R_{2}$ in Fig. 6.9b and 6.9d). A similar behavior can be observed in Fig. 6.11a, 6.11c, and 6.11e for the real flight experiments. The capabilities of the list of missions implemented in the GMP acquire an utmost importance in the second real flight experiment shown in Fig. 6.11c and 6.11d, In 
this experiment, the proposed mission planning system should manage the simultaneous target detection events that are produced while detecting simultaneously both buckets using the image captured from the front camera of the UAV (see image A in Fig. 6.11d). At this point of the mission, the GMP has an active list composed of three missions: the main exploration mission, and two rescue missions for both detected targets. As shown in Fig. 6.11d, the GMP is able to correctly manage this situation by planning first the rescue operation of bucket $A$, then the rescue of bucket $B$, and finally recovering the exploration mission. It should be emphasized the outstanding pose estimation obtained using a Perspective- $n$-Point $(\mathrm{P} n \mathrm{P})$ algorithm with Levenberg-Marquardt optimization and RANSAC for filtering the outliers, which allows obtaining a proper mapping of the detected targets for a precise rescue mission.

Regarding the results obtained for the SAR mission conducted in the third real-flight scenario, it should be noted that for this experiment, the classical object detection algorithm based on shape an color information used for the first and second experiments, is replaced in this case by a perception component based on deep learning techniques (see Appendix B) integrated into the Model-Based Object Detector component (see Section 6.4.4. Moreover, the Geometry-based IBVS controller (see Section 6.4.5) used for the first and second experiments is replaced by the RL-IBVS algorithm presented in Chapter 4. Despite using different components for the perception and rescue tasks, the results presented in Fig. 6.11e and 6.11f reveal the modularity of our Aerostack architecture, and also of the proposed mission planning system. This feature is benefited by the use of ROS, which provides an useful and generic interface for communication between the different components, regardless of their nature. The results presented in Fig. 6.11e and 6.11f reveal the appropriate behavior of the RL-IBVS, which allowed the correct rescue operation on the static targets. In this mission, the accurate detection of the object in the image plane represents a critical step for pose estimation purposes and the subsequent rescue operation on both targets. The pose estimation of the targets, addressed by using a $\mathrm{P} n \mathrm{P}$ algorithm, provides an acceptable pose estimation using monocular information. During the execution of the real flight SAR mission of Fig. 6.11f, the average errors in the position estimation of both targets were $[x: 0.19 \mathrm{~m}, y: 0.08 \mathrm{~m}, z: 0.062 \mathrm{~m}]$, highlighting the low errors obtained in the estimation of the $y$ and $z$ coordinates, both under $8 \mathrm{~cm}$. The error in the $x$ coordinate is slightly higher, which can be caused by sudden roll movements of the UAV while detecting the target. These errors are adequate for SAR missions and have low effect on our system since a fisheye-lens bottom camera with a considerable field of view is utilized. This field of view allows the detection of the target within an area of 4 $\mathrm{m} \times 4 \mathrm{~m}$ at the flight altitude of $1.2 \mathrm{~m}$. Furthermore, the proposed RL-IBVS can handle quite big errors in the detection as it has been demonstrated throughout the experiments of Chapter 4.

Overall, it should be remarked the versatility and modularity of the proposed mission planning system which has been demonstrated during the SAR experiments presented in this section. The results obtained in these experiments have shown how the proposed system is able to efficiently switch between control modes (i.e., from position control mode while navigating through the mission points, to velocity control mode when using the proposed RL-IBVS for rescue purposes). Besides, these results also reveal the efficient 
transition between perception devices. During the SAR experiments, once the object has been detected using the front camera, the proposed system changes the perception mode for using the bottom camera in order to perform the rescue operation. Once the rescue operation is finished, the proposed system efficiently recovers the perception mode using the front camera for exploration purposes.

\subsection{Conclusions}

In this chapter, a versatile mission planning system has been presented. The proposed mission planning system integrates a Global Mission Planner (GMP), which is responsible for the mission generation and mission re-planning in response of possible events that can occur during the execution of the mission, and an Agent Mission Planner (AMP), which is in charge of scheduling task by task the mission provided by the GMP, and creates a communication interface between the high-level mission defined by the GMP and the actions executed for accomplishing the mission. The main capabilities of the proposed mission planning system, derived from the results presented throughout this chapter, are highlighted next:

- The sampling strategy and the mission point distribution algorithm implemented within the GMP provide an efficient solution for the exploration of unknown indoor environments in order to accomplish high-level missions (e.g., SAR) using a single or a muti-agent configuration.

- One of the most important capabilities of the proposed GMP is the dynamic mission re-planning. For this purpose, this component has an active list of missions, which allows the concatenation of different missions in response to the wide range of events that can occur during the execution of complex missions, such as SAR (e.g., a target has been detected and a rescue mission is planned).

- One of the most important capabilities implemented within the AMP is the dynamic mission point reallocation. Since the scenario to be explored is unknown in advance, the mission points automatically generated by the GMP can fall within an obstacle. This situation is detected by the Path Planner component, which sends the corresponding event to the AMP. The latter implements an iterative algorithm for mission point reallocation considering a safety circumference based on the inflation radius of the obstacles. This capability provides a higher level of flexibility to the proposed mission planning system.

Overall, the flexible design of the proposed mission planning system allows the execution of very complex missions using a single or a multi-agent configuration. The accomplishment of such missions is benefited by the user-friendly design of the GMP, which allows the definition of the mission, task by task, using a simple XML-based language, or the automatic mission generation where the human operator only provides a high-level command, such as find target, explore, SAR, etc. 


\section{Chapter 7}

\section{Conclusions and Future Work}

\subsection{Conclusions}

In this thesis, we have explored and thoroughly analyzed the performance of multiple learning-based algorithms in different components of an aerial robotic architecture, such as perception, visual control, navigation, and planning.

The results presented throughout this thesis demonstrate the power of current AI techniques, and more concretely the power of machine learning techniques. These techniques have proven to provide outstanding results in a wide variety of problems within the robotics field. More concretely, within the paradigm of supervised and semi-supervised learning, the proposed Fully Convolutional Networks (FCNs) have shown outstanding results in electric tower detection and insulator string segmentation. On the other hand, the proposed Siamese Convolutional Neural Networks (SCNNs) and the Siamese Convolutional Autoencoder (SCAE) in combination with the One-class SVM, have shown outstanding results in the diagnosis of faults in electrical insulator strings. Regarding the reinforcement learning paradigm, in this thesis we have designed and evaluated different algorithms based on deep reinforcement learning techniques for image-based visual servoing and reactive navigation tasks. The results obtained show the potential of RL techniques for learning complex tasks in robotics. Furthermore, the proposed deep RL algorithms have been completely trained in simulation and tested in simulation and realflight experiments. This fact opens a wide research direction for exploring new and more advanced robotics simulators in which complex behaviors can be learned by interacting with the environment, that is, learning from experience. Learning in simulation environments can be of particular interest in the field of aerial robotics, where learning from experience in real-flight conditions can be extremely hard, and to the author's knowledge has not been yet addressed in the literature.

From the author's point of view, classic techniques in some engineering processes applied to solve computer vision tasks, control, and navigation, are extremely powerful when the specifications of the environment (e.g., structured light conditions in computer vision) or the system (e.g., the dynamics of the robotic platform) are perfectly known. In these situations, the knowledge of the system and its environment justifies the usage of classic techniques, which are well-known in science and engineering. However, in 
most conditions, even this knowledge of the system is then translated into mathematical approximations (e.g., mathematical simplifications in order to model the dynamics of a system, linearization around an operating point in classical control theory, etc.). Learning-based techniques are extremely powerful since they learn from data. Nowadays, in the era of information, we have tons of data collected during years. Furthermore, with the growth in computational resources, more powerful simulators are built. This justifies and allows the application of a wide range of machine learning techniques, and even more complex models within the field of Deep Learning. Besides, learning-based techniques provide some non-linearities that are applied to the data and thus, these techniques can learn extremely complex behaviors, very difficult to model, in some cases, with traditional science and engineering procedures.

Learning-based techniques are increasingly being established in the science and engineering processes. In critical systems (e.g., the aerospace sector) the control of the systems carried out with techniques totally based on learning is still far from being a reality. However, even in these critical systems, learning-based techniques can be used in combination with classical ones.

\subsection{Future Work}

Future work is aligned towards the exploration of the limits of AI techniques, and more concretely learning-based techniques, with special interest on their application in the robotics field. Furthermore, the combination of consolidated engineering methods with AI techniques as mentioned in the previous section can provide extremely good results since these systems can benefit from the capabilities of both approaches. Thus, this combination should be further explored in different components of a robotic agent such as the ones studied in this thesis.

On the other hand, the extension of the proposed learning-based approaches, with special interest in the ones involving reinforcement learning techniques, to different robotic platforms (e.g., ground robots) will be considered in future works. Besides, the usage of other types of sensors (e.g., RGB-D cameras) will be analyzed for the adaptation of the reactive navigation approaches presented in this thesis. Additional extensions of the proposed reinforcement learning algorithms can involve the application of Imitation Learning or Reinforced Imitation Learning techniques, which are gaining significant importance within the research community in recent years.

Finally, it should be emphasized the current trend in the research community consisting of training learning-based algorithms using robotics simulators. In this direction, we consider as a promising research line the use of Domain Adaptation techniques. More concretely, the application of GANs for adapting the domain from simulation to real conditions and vice versa could benefit the training process of the robotic agents in order to learn complex behaviors for real world applications. From the author's point of view, research in the development of photo-realistic simulators can lead to significant advances in the field of deep learning since a massive amount of data can be generated for training purposes. 
Appendices 



\section{Appendix $\Lambda$}

\section{Deep Learning Background}

In this Appendix, the objective is to provide a better intuition of the different algorithms and techniques involved in the training process of Deep Neural Networks (DNNs) and Deep Reinforcement Learning (DRL) algorithms in order to provide a better understanding of the learning paradigms used throughout this thesis. For this purpose, in Section A.1 we provide an intuitive analysis of the main techniques used in the training process of DNNs, with a special focus on the ones used in this thesis. Subsequently, Section A.2 focuses on the mathematical aspects and intuitions of the DRL algorithms applied in this thesis.

\section{A.1 Deep Neural Networks}

\section{A.1.1 Basics in Convolutional Neural Networks}

Deep Neural Networks (DNNs) are built by stacking several hidden layers. Each hidden layer applies a non-linear activation function to the weighted output of the previous layer. The activation of unit $k$ in layer $m$ given its $n$ inputs (outputs of the previous layer $m-1$ ) is computed using Eq. A.1.

$$
a_{k}^{m}=g\left(\omega_{k 0}^{m-1} a_{0}^{m-1}+\omega_{k 1}^{m-1} a_{1}^{m-1}+\ldots+\omega_{k n}^{m-1} a_{n}^{m-1}\right)
$$

where $g$ is typically a non-linear activation function (e.g., tanh, sigmoid, ReLU, etc.), $\mathbf{a}^{m-1}$ is the output from the previous layer, and $\boldsymbol{\omega}_{k}^{m-1}$ represents the vector of weights of layer $m-1$ for unit $k$ to be learned by the network.

In this thesis, most of the DNN architectures designed consist of Convolutional Neural Networks (CNNs), which are a specific type of DNNs, built by stacking several convolutional, pooling, and fully-connected layers (in their basic form). The convolutional layers are responsible for extracting the features in the image by learning several convolution filters or kernels which will be convolved over the input image and subsequent feature maps in order to learn data representations. In CNNs, the most representative operation is the $2 \mathrm{D}$ convolution of a two-dimensional image $I$ with a two-dimensional kernel $K$, 
given by Eq. A.2.

$$
C(i, j)=(I * K)(i, j)=\sum_{m} \sum_{n} I(m, n) K(i-m, j-n)
$$

An important functionality of convolutional layers is the parameter sharing. This means that the weights learned for a specific kernel $K$ are applied to all the locations of the image $I$, avoiding the necessity of learning a different set of weights for each location in the image. This functionality provides the convolutional layers an interesting property called equivariance to translation, which means that if the input changes, the output provided by the convolutional layer changes in the same way. Subsequently, each convolutional layer in the $\mathrm{CNN}$ is usually followed by a non-linear activation function, where Rectified Linear Units (ReLUs), proposed in Nair and Hinton, 2010, are the most common choice due to the good properties of their activation function $(g(z)=\max (0, z))$ which allows to speed up the learning process and deal with the issue of vanishing gradients.

The pooling layers are usually inserted in between the convolutional layers, and provide an important reduction of the number of parameters of the neural network by progressively reducing the spatial resolution of the feature maps, thus preventing overfitting problems. The most common pooling operation is the max pooling, which takes the maximum value of a defined rectangular neighborhood and discards the rest of values. Other popular choice of pooling function is the average pooling operation, in which the average of a rectangular neighborhood is computed. In all cases, the pooling operation provides representations that are approximately invariant to small translations of the input (Goodfellow et al., 2016).

Finally, fully-connected layers act as a standard feed-forward neural network, where each unit in the layer is connected to every unit in the preceding layer. These layers are usually designed as the final layers of standard CNNs, in which the output layer of the fully-connected network provides the final classification or regression prediction.

\section{A.1.2 Activation Functions}

Although a large number of non-linear functions have been designed for operating in DNNs (Nwankpa et al., 2018), some of these functions have been widely adopted in the research community, such as the well-known ReLU activation function, or its extended versions: Leaky ReLU (Maas et al., 2013) and PReLU (He et al., 2015a). The nonlinearities implemented by these activation functions provide some properties that benefit the learning process of the DNNs, mitigating important problems associated with very deep networks such as the problem of vanishing gradients. In this section, we provide an intuition of these benefits as compared to other standard non-linearities such as the sigmoid or tangent hyperbolic (tanh) functions. In this thesis, the great majority of activation functions used in the hidden layers of the proposed DNNs consist of ReLU activation functions, using the sigmoid and tanh for the prediction conducted on the output layer of the networks.

In most of the current deep neural networks, the learning process is guided using gradient descent and its variants (e.g., stochastic gradient descent). In this process, the 
computation of the gradients is usually conducted using the backpropagation algorithm (Rumelhart et al., 1986), which originated an enormous breakthrough in the training process of neural networks. In the backpropagation algorithm, the chain rule is applied in order to compute the gradients of the objective function of the network (i.e., the loss function) with respect to the corresponding weights at each layer. The chain rule implies a sequence of gradients multiplications, which can easily cause problems such as vanishing or exploding gradients. The former is produced when the gradients tend progressively to very small values (i.e., close to 0), which can cause the stop of the learning process in the first layers of the network. Some activation functions, such as the sigmoid or the tanh are more prone to suffer from vanishing gradients due to their derivative. As shown in Fig. A.1, the derivative function of the sigmoid and tanh functions tend rapidly to 0 when the logit (non-normalized prediction of the corresponding neuron, i.e., $z=\mathbf{w}^{T} \mathbf{x}+b$ ) starts increasing in absolute value. This effect is even stronger in the case of the sigmoid function, since its derivative has a maximum value of 0.25 (see Fig. A.1a), which intuitively can be more prone to vanish the gradients when backpropagation is applied. In contrast, the derivative provided by the ReLU function (see Fig. A.1c) acquires a constant value of 1 for any value of the logit greater than 0 (i.e., $z>0$ ), which eliminates the vanishing gradients problem, and allows training very deep networks. Furthermore, ReLU activations allow the network to achieve sparse representations of the data (Glorot et al., 2011) and are less computationally expensive than sigmoid or tanh functions. The latter property can be of utmost importance in the robotics field where very deep neural networks might run on the onboard computer of the robot.

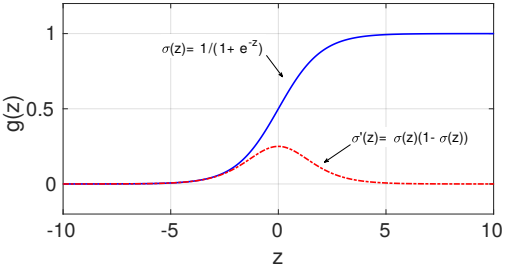

(a)

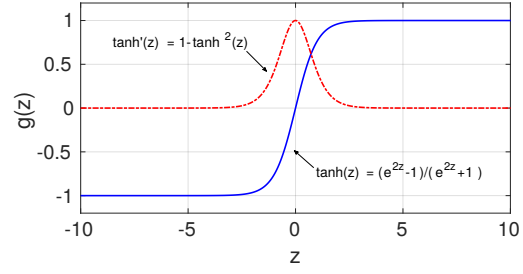

(b)

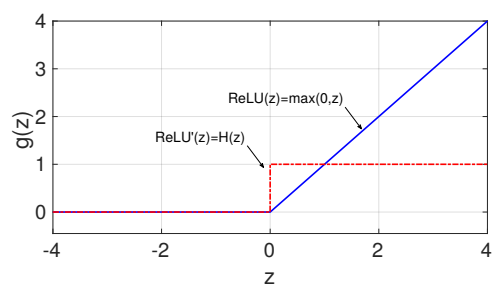

(c)

Figure A.1: Usual neural network activation functions and their derivatives. (a) Sigmoid activation function and its derivative. (b) tanh activation function and its derivative. (c) ReLU activation function and its derivative. $H(z)$ represents the Heaviside function.

Despite the good properties exhibited by the ReLU activation function for dealing with the problem of vanishing gradients, it is important to highlight some problems that this activation can introduce to the network. The most important problem is usually called "dying" ReLU, and its intuition is easy to understand when analyzing Fig. A.1c. As shown in this figure, the inputs to the ReLU function can cause the displacement to the negative side of the curve (i.e, $z<0$ ). In this situation, since the gradient of the ReLU function is also 0 , the neuron stops contributing to the learning process and it no longer recovers. For mitigating the "dying" ReLU problem, several alternative functions have been proposed in the literature such as the already mentioned Leaky $\operatorname{ReLU}(g(z)=\max (0.01 z, z))$ and $\operatorname{PReLU}(g(z)=\max (0, z)+\alpha \min (0, z))$, which introduce a small slope for negative values. 
On the other hand, in multi-class classification problems, the final fully-connected layer contains one unit per target class in the model, and usually has a softmax activation function (see Eq. A.3), which normalizes the input vector of $N$ entries into a probability distribution. In this way, we can map the $N$ entries of a vector into an interpretable probability vector, where each entry is within the interval $[0,1]$ and their values sum up to one.

$$
g(\mathbf{z})_{j}=\frac{\exp \left(z_{j}\right)}{\sum_{n=1}^{N} \exp \left(z_{n}\right)}
$$

where $j$ is the actual class, and $N$ represents the total number of classes.

\section{A.1.3 Optimization}

In this section, we provide the intuition behind the mathematical formulation of the optimization algorithm that has been utilized in the training process of most of the networks proposed in this thesis, that is, the Adaptive Moment Estimation (Adam) optimization algorithm (Kingma and Ba, 2014), which combines the benefits of AdaGrad (Duchi et al., 2011) and RMSProp (Tieleman and Hinton, 2012) algorithms. For this purpose, we first introduce the concept of exponentially weighted moving average (EWMA). EWMA acts as a first-order infinite impulse response filter which applies a moving average of past samples taking into account weighting factors that decrease exponentially. In the machine learning field, the concept of EWMA has been widely applied in the context of optimization algorithms such as RMSProp and Adam, and is also utilized in several deep reinforcement learning techniques as described in Section A.2. The basic formulation of EWMA is governed by Eq. A.4, which can be rewritten as a function of all the previous time steps using Eq. A.5.

$$
\begin{aligned}
m_{t} & =\beta \cdot m_{t-1}+(1-\beta) \cdot y_{t} \\
m_{t} & =(1-\beta) \sum_{i=1}^{t} \beta^{t-i} y_{i} \\
& =(1-\beta) \cdot y_{t}+(1-\beta) \beta \cdot y_{t-1}+(1-\beta) \beta^{2} \cdot y_{t-2}+\cdots+(1-\beta) \beta^{t-1} \cdot y_{1}
\end{aligned}
$$

where $m_{t}$ is the result of the EWMA at time step $t, \beta$ is the weighting decaying factor, and $y_{t}$ is the value of the function over which the EWMA is applied at time step $t$.

The analysis of Eq. A.5 reveals the behavior of the EWMA, where the samples away from the current time step $t$ are weighted exponentially with a progressively decaying contribution to the value of $m_{t}$. An example of the effect of EWMA using two values of $\beta$ can be observed in Fig. A.2. In this example, we illustrate the effect of EWMA on a sine function with added noise in order to see the effect of averaging with respect to past samples in the sequence. As shown in Fig. A.2, using a factor of $\beta=0.9$ allows for providing a smooth response since we are averaging taking into account a large number of past samples (see Fig. A.2b). Thus, $m_{t}$ is less responsive since the average in conducted on a larger window, which produces a shifting effect, as can be observed in Fig. A.2a, 
where $m_{t}$ for $\beta=0.9$ is shifted to the right (see black line in Fig. A.2a). In contrast, using a lower value of $\beta$, for example $\beta=0.6$ produces a more responsive, but also more oscillatory response (see green line in Fig. A.2a).

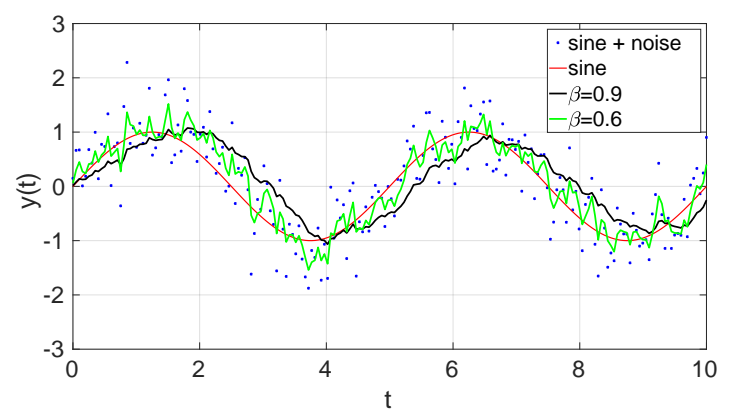

(a)

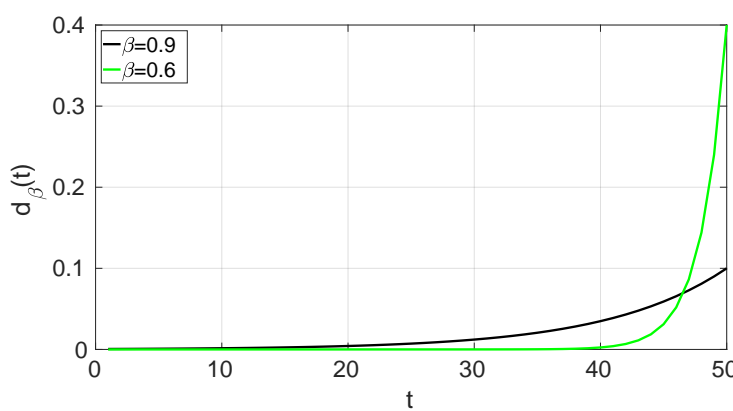

(b)

Figure A.2: Exponentially Weighted Moving Average intuition. (a) Example of the effect of EWMA on a sine function using two values of $\beta$. (b) Decay effect $\left(d_{\beta}(t)=(1-\beta) \beta^{t-i}, \quad i=\right.$ $1, \ldots, t)$ caused by each $\beta$ decaying factor for a temporal horizon of $t=50$.

The concept of EWMA is widely used in gradient-based optimization methods such as Stochastic Gradient Descent (SGD) with momentum (Sutskever et al., 2013), and RMSProp. In these algorithms, the desired effect of EWMA is to reduce the oscillations of gradient descent during the learning process. In addition to EWMA, RMSProp introduces a root squared term in the update term of the SGD (see Eq. A.7), computed over the EWMA of the second moment estimates of the gradient. In order to illustrate this concept, let's consider the derivative of the loss function with respect to the parameters of our network as $G=\partial \mathcal{L} / \partial \boldsymbol{\omega}$. Then, the equations of the SGD method using RMSProp can be written as shown in Eq. A.6 and Eq. A.7.

$$
\begin{aligned}
m s_{t} & =\beta \cdot m s_{t-1}+(1-\beta) \cdot G_{t}^{2} \\
\omega_{t} & =\omega_{t-1}-\alpha \frac{G_{t}}{\sqrt{m s_{t}}}
\end{aligned}
$$

where $m s_{t}$ is the moving average of the squared gradient for each weight at time step t. $G_{t}^{2}$ represents the Hadamard product $\left(G_{t} \odot G_{t}\right)$, and $\alpha$ is the learning rate.

The analysis of Eq. A.6 and Eq. A.7, reveals the additional property provided by the RMSProp to damper the oscillations caused by the gradient descent method during the learning process. These oscillations are not only reduced when considering the EWMA implemented in Eq. A.6, but also due to the denominator introduced in Eq. A.7. Intuitively, when the gradient in a specific direction is large, then $m s_{t}$ can become large, preventing the update term in Eq. A.7 to take large values. Conversely, when the gradient is small, then $m s_{t}$ can become small, and thus the update term in Eq. A.7 gets amplified, allowing to accelerate the learning process in that specific direction.

Finally, the Adam algorithm combines the properties of SGD with momentum and RMSProp to provide a more stable learning algorithm, which has shown to work well in 
a wide range of applications. Adapted from Kingma and Ba, 2014, in Algorithm 4 we illustrate the basic steps of the Adam method for a better understanding. As shown in Algorithm 4, the Adam method combines the concepts of EWMA (i.e., analogous to the concept of momentum in gradient-based learning) and RMSProp, incorporating additional steps for correcting the biased estimates of the first and second moments (see lines 9 and 10 in Algorithm 4). Since the initialization of the EWMA is usually conducted using a vector of $0^{\prime} s$, then the estimation of the EWMA in the initial steps of the learning process is biased towards 0 . In order to eliminate this bias, the term $\left(1-\beta^{t}\right)$ is introduced, which helps to obtain a better estimate of the moving averages during the initial phase of the learning process. Intuitively, when $t$ is small (i.e., in the initial steps of the learning process), the moving average (e.g., $\left.m_{t}\right)$ gets divided by a small number and thus it gets amplified. Conversely, when $t$ starts increasing, the term $\beta^{t}$ progressively approximates to 0 , and then the moving average gets divided by 1 .

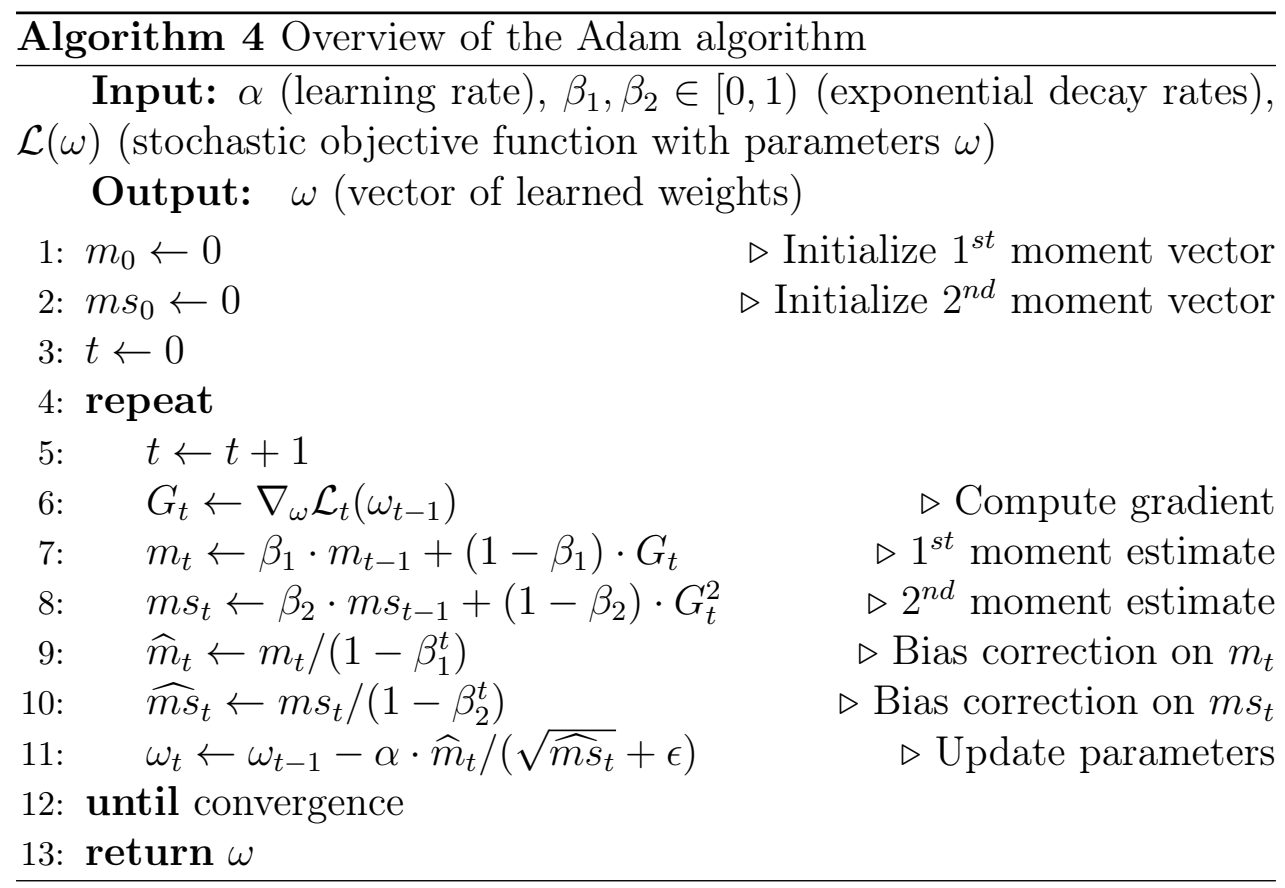

Finally, apart from the desirable properties of the Adam optimizer in terms of stabilization of the learning process (i.e., dampening the oscillations during learning), it should be emphasized that this method is also computationally efficient in terms of memory consumption, since for computing an EWMA only a raw number is stored in memory, which is overwritten in every iteration of the learning process. Furthermore, it should be remarked that all these methods that involve the computation of EWMA (e.g., SGD with momentum, RSMProp, Adam) allow (in general terms) to choose greater learning rates with the consequent acceleration of the learning process, since the oscillations produced by the gradient descent during learning are usually dampened in the direction of greater variance. 


\section{A.2 Deep Reinforcement Learning}

The main components in the reinforcement learning paradigm are the agent and the environment. The final objective is to train the agent in order to find the policy that maximizes the accumulated discounted reward $R_{t}=\sum_{i=t}^{T} \gamma^{(i-t)} r\left(\mathbf{s}_{i}, \mathbf{a}_{i}\right)$, given the state $\mathbf{s}_{i}$, the action $\mathbf{a}_{i}$, and a discount factor $\gamma \in[0,1]$ (Sutton et al., 1998). This policy represents a mapping function from state $\mathbf{s} \in \mathbb{S}$ to an action $\mathbf{a} \in \mathbb{A}$, where $\mathbb{S}$ is the state space and $\mathbb{A}$ is the finite action space. For this purpose, Deep Reinforcement Learning algorithms (Mnih et al., 2015, Lillicrap et al., 2015, Mnih et al., 2016, Gu et al., 2016) have proven to provide outstanding results.

The Deep Deterministic Policy Gradients (DDPG) algorithm (Lillicrap et al., 2015) is a model-free, off-policy algorithm based on an actor-critic architecture, which combines the functionalities of the Deep Q-Network (DQN) proposed in Mnih et al., 2015 and the Deterministic Policy Gradient (DPG) introduced in Silver et al., 2014. Among the main contributions of the DQN we can highlight the introduction of the experience replay technique for breaking the correlations between observations of the agent, and the use of a separate target $Q$ network for breaking the correlations between the action-values (Q) and the target values $y_{t}$ (see Eq. A.10). In Silver et al., 2014, DPG algorithms were proven to be an effective strategy for learning a deterministic target policy following a separate stochastic behavior policy for exploration, where the deterministic policy gradient was efficiently computed as the expected gradient of the action-value function.

Regarding the DDPG algorithm, the critic uses a neural network as a nonlinear function approximator for estimating the action-value function, which is usually computed using the Q-value Bellman equation shown in Eq. A.8 when the policy is deterministic.

$$
Q^{\mu}\left(\mathbf{s}_{t}, \mathbf{a}_{t}\right)=\mathbb{E}_{r_{t}, \mathbf{s}_{t+1} \sim E}\left[r\left(\mathbf{s}_{t}, \mathbf{a}_{t}\right)+\gamma Q^{\mu}\left(\mathbf{s}_{t+1}, \mu\left(\mathbf{s}_{t+1}\right)\right)\right]
$$

where $Q^{\mu}\left(\mathbf{s}_{t}, \mathbf{a}_{t}\right)$ is the action-value function, $r\left(\mathbf{s}_{t}, \mathbf{a}_{t}\right)$ is the immediate reward, $\gamma$ is the discount factor, and $\mu$ is the learned policy given by the actor network.

The learning process of the critic is performed by minimizing the loss function given by Eq. A.9, whose gradient is used for updating the weights of the critic network $\left(\omega^{Q}\right)$.

$$
\mathcal{L}\left(\omega^{Q}\right)=\mathbb{E}_{\mu^{\prime}}\left[\left(Q\left(\mathbf{s}_{t}, \mathbf{a}_{t} \mid \omega^{Q}\right)-y_{t}\right)^{2}\right]
$$

where

$$
y_{t}=r_{t}+\gamma Q^{\prime}\left(\mathbf{s}_{t+1}, \mu^{\prime}\left(\mathbf{s}_{t+1} \mid \omega^{\mu^{\prime}}\right) \mid \omega^{Q^{\prime}}\right)
$$

is the target value computed taking into account the action generated by the target actor network $\left(\mu^{\prime}\right)$ and the action-value function estimated by the target critic network $\left(Q^{\prime}\right)$.

As an off-policy algorithm, the exploration in the DDPG is conducted by means of a stochastic exploration policy computed using Eq. A.11, which allows learning independently a deterministic target policy determined by the actor. The actor network acts deterministically by mapping observations from the agent to actions.

$$
\mu^{e}\left(\mathbf{s}_{t}\right)=\mu\left(\mathbf{s}_{t} \mid \omega_{t}^{\mu}\right)+\mathcal{N}
$$

where $\mathcal{N}$ is the distribution generated by an Ornstein-Uhlenbeck process. 
Finally, the weights of the actor network are updated by using Eq. A.12.

$$
\begin{aligned}
\nabla_{\omega^{\mu}} \mu & \approx \mathbb{E}_{\mu^{\prime}}\left[\left.\nabla_{\omega^{\mu}} Q\left(\mathbf{s}, \mathbf{a} \mid \omega^{Q}\right)\right|_{\mathbf{s}=\mathbf{s}_{t}, \mathbf{a}=\mu\left(\mathbf{s}_{t} \mid \omega^{\mu}\right)}\right] \\
& =\mathbb{E}_{\mu^{\prime}}\left[\left.\left.\nabla_{\mathbf{a}} Q\left(\mathbf{s}, \mathbf{a} \mid \omega^{Q}\right)\right|_{\mathbf{s}=\mathbf{s}_{t}, \mathbf{a}=\mu\left(\mathbf{s}_{t}\right)} \nabla_{\omega^{\mu}} \mu\left(\mathbf{s} \mid \omega^{\mu}\right)\right|_{\mathbf{s}=\mathbf{s}_{t}}\right]
\end{aligned}
$$

Other remarkable contribution of the DDPG algorithm is the soft update method used for updating the weights of the target actor $\left(\mu^{\prime}\left(s \mid \omega^{\mu^{\prime}}\right)\right)$ and target critic $\left(Q^{\prime}\left(s, a \mid \omega^{Q^{\prime}}\right)\right)$ computed using Eq. A.13, where again the concept of EWMA is utilized.

$$
\begin{aligned}
\omega^{Q^{\prime}} & =\tau \omega^{Q}+(1-\tau) \omega^{Q^{\prime}} \\
\omega^{\mu^{\prime}} & =\tau \omega^{\mu}+(1-\tau) \omega^{\mu^{\prime}}
\end{aligned}
$$

where $\tau \ll 1$ stabilizes the learning process, moving the problem of learning the action-value function towards the supervised learning paradigm.

As observed in the DDPG algorithm, two different function approximators (i.e., the actor and critic neural networks) are trained using two different objective functions. In contrast, the authors in $\mathrm{Gu}$ et al., 2016 proposed a Normalized Advantage Functions (NAF) algorithm, which is built on top of standard Q-learning and uses only a single neural network (not taking into account the target network) for computing the value function and the policy. In order to provide a complete understanding of the NAF algorithm, we define next the value function $\left(V^{\pi}\left(\mathbf{s}_{t}\right)\right)$ and the advantage function $\left(A^{\pi}\left(\mathbf{s}_{t}, \mathbf{a}_{t}\right)\right)$, which are given by Eq. A.14 and Eq. A.15, respectively.

$$
\begin{gathered}
V^{\pi}\left(\mathbf{s}_{t}\right)=\mathbb{E}_{r_{i \geq t}, s_{i>t} \sim E, a_{i \geq t} \sim \pi}\left[R_{t} \mid \mathbf{s}_{t}\right] \\
A^{\pi}\left(\mathbf{s}_{t}, \mathbf{a}_{t}\right)=Q^{\pi}\left(\mathbf{s}_{t}, \mathbf{a}_{t}\right)-V^{\pi}\left(\mathbf{s}_{t}\right)
\end{gathered}
$$

An alternative representation of the advantage function is used in the NAF algorithm in order to apply Q-learning with experience replay to continuous tasks (see Eq. A.16).

$$
A\left(\mathbf{s}, \mathbf{a} \mid \omega^{A}\right)=-\frac{1}{2}\left(\mathbf{a}-\boldsymbol{\mu}\left(\mathbf{s} \mid \omega^{\mu}\right)\right)^{T} \cdot \mathbf{P}\left(\mathbf{s} \mid \omega^{P}\right) \cdot\left(\mathbf{a}-\boldsymbol{\mu}\left(\mathbf{s} \mid \omega^{\mu}\right)\right)
$$

where $\mathbf{a}=\boldsymbol{\mu}\left(\mathbf{s} \mid \omega^{\mu}\right)+\mathcal{N}$, is the action generated by adding noise using an OrnsteinUhlenbeck distribution to the policy predicted by the network, $\mathbf{P}\left(\mathbf{s} \mid \omega^{P}\right)$ is a positive definite squared matrix given by: $\mathbf{P}\left(\mathbf{s} \mid \omega^{P}\right)=\mathbf{L}\left(\mathbf{s} \mid \omega^{P}\right) \cdot \mathbf{L}\left(\mathbf{s} \mid \omega^{P}\right)^{T}$, where $\mathbf{L}\left(\mathbf{s} \mid \omega^{P}\right)$ is a lower-triangular matrix, whose values are also predicted by an output linear unit of the network.

Based on the previous formulation, the NAF algorithm combines the deep Q-learning algorithm in Mnih et al., 2015 with the same experience replay method and target network update as in the DDPG algorithm. That is, the objective is to minimize the loss defined in Eq. A.9 using the action-value function: $Q\left(\mathbf{s}, \mathbf{a} \mid \omega^{Q}\right)=A\left(\mathbf{s}, \mathbf{a} \mid \omega^{A}\right)+V\left(\mathbf{s} \mid \omega^{V}\right)$, and the target computed in Eq. A.17.

$$
y_{t}=r_{t}+\gamma V^{\prime}\left(\mathbf{s}_{t+1} \mid \omega^{Q^{\prime}}\right)
$$


where $V^{\prime}\left(\mathbf{s}_{t+1} \mid \omega^{Q^{\prime}}\right)$ is the value function predicted by the target network with parameters $\omega^{Q^{\prime}}$.

In summary, as compared to the actor-critic architecture of the DDPG, the NAF algorithm uses a simpler formulation based on normalized advantage functions, which avoids the necessity of using a second actor network. The proposed neural network in the NAF formulation is used to simultaneously predict the value function, the policy, and an advantage term used to compute the advantage function. Both NAF and DDPG algorithms used in this thesis, have demonstrated the effectiveness of model-free, off-policy DRL algorithms applied for learning complex tasks in the robotics field, where the highdimensional state space and the continuous action domain complicate the use of classical RL algorithms. 



\section{Appendix}

\section{Additional Object Detection Results}

In this appendix we present additional results obtained for the detection of specific objects, such as buckets, which was the main use case in the IMAV 2016 competition ${ }^{1}$ for accomplishing SAR missions. The main algorithm implemented in this case is an adaptation of the Algorithm 1 presented in Section 2.3, where the region proposal module is replaced by another algorithm which generates proposals based on the information of the buckets to be detected, such as color (i.e., buckets can be red or blue) and aspect ratio (i.e., buckets are cylindrical objects of $0.3 \mathrm{~m}$ height and $0.25 \mathrm{~m}$ of diameter). The rest of the object detection algorithm is the same as described in Section 2.3. In addition, we add Convolutional Neural Networks (CNNs) for enlarging the comparison between supervised learning classifiers.

The above setup was used in the IMAV 2016 competition and subsequent experiments included in Sampedro et al., 2018b, which are summarized here.

\section{B.1 Image Dataset}

In the SAR missions proposed for evaluating our system, the targets consist of cylindrical buckets of different colors positioned at random locations in the scenario. Currently, there are no publicly available datasets containing images of cylindrical buckets that could be used for training a deep learning classifier. For this purpose, a custom dataset has been created containing images from background (non bucket) class and bucket class. After a large process of data acquisition, a total of 875 images for the bucket class and 2250 images for the background class were collected (see Fig. B.1 for an example).

From this original dataset, several data augmentation techniques have been applied in order to increase the number of images used for training and evaluating the classifier and to prevent overfitting problems. The data augmentation process consisted in the application of three main techniques: random cropping, horizontal flipping, and noise addition. The random cropping strategy was similar to the one presented in Krizhevsky et al., 2012, obtaining four cropped images per original image, by selecting a random offset starting from each of the four corners in the original image. The horizontal flipping strategy con-

\footnotetext{
${ }^{1}$ http://www.imavs.org/2016/
} 

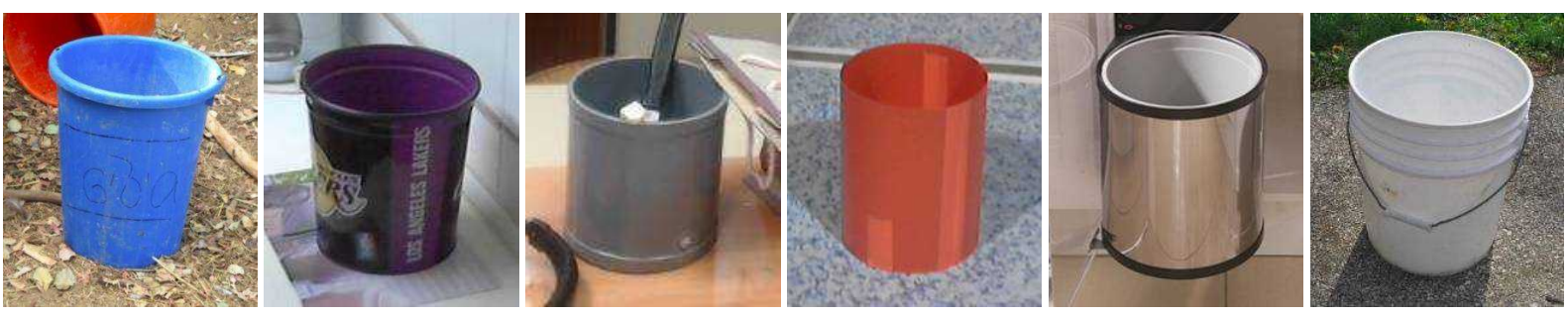

(a)
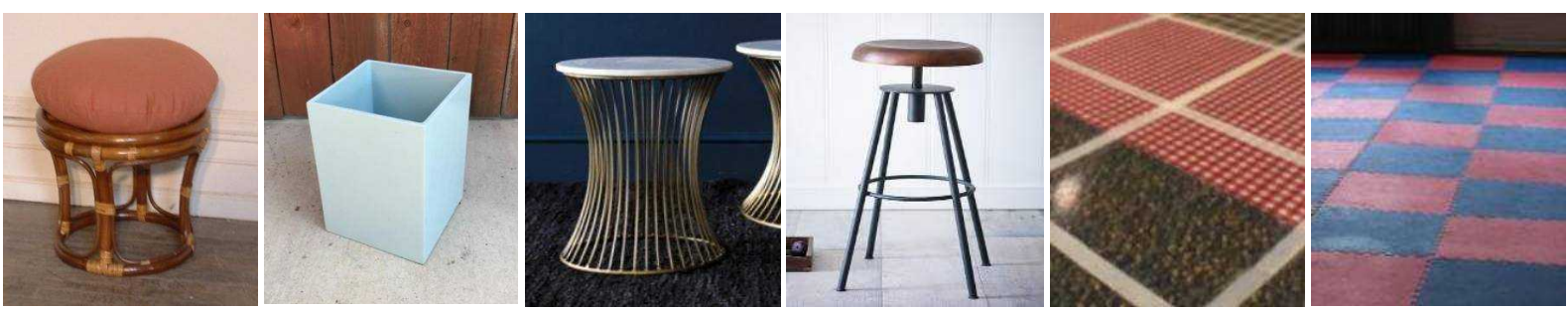

(b)

Figure B.1: Examples of images used for training the supervised learning classifiers for bucket/background classification. (a) Cylindrical bucket training examples. (b) Background (non bucket) training examples. Images of office furniture and different types of floors have been used as examples of the background class.

sisted in mirroring the original image from left to right direction, which allowed doubling the number of images. Finally, the last data augmentation technique was based on adding Gaussian noise to the original image with zero mean and a standard deviation of 10 pixels. From the 875 images of bucket class, we performed data augmentation over 375 of these images obtaining a total of 4500 images. The remaining 500 images were added to the augmented dataset, providing a total amount of 5000 images for bucket class. From the 2250 images belonging to background class, data augmentation was conducted over 550 of these images, obtaining a total of 3300 images. The remaining 1700 images were added to the augmented set, providing a total amount of 5000 images for background class.

Subsequently, in order to train and evaluate the supervised learning classifiers considered in this work, the final dataset consisting of 10000 images, was divided into train (70 $\%)$, validation $(15 \%)$ and test $(15 \%)$ sets.

\section{B.2 Training Methodology and Evaluation}

In order to conduct a reliable comparison between the different supervised learning classifiers considered for bucket/background classification, a similar methodology as the one presented in Caruana and Niculescu-Mizil, 2006 has been applied: First, a total of 6 evaluation tests have been defined, where the train, validation and test sets were randomly picked for each evaluation test. In each of the 6 evaluation tests, a 5-fold crossvalidation procedure is performed in the case of the L2R-LR and L-SVM classifiers in order to select the optimal regularization parameters of the corresponding classifier (see Eq. 2.3 and 2.4 in Section 2.5.4). The range of values considered for the regularization 
parameter covers a wide range starting from $10^{-4}$ to $10^{2}$ with a step of $2 \times 10^{p}$, where $p$ ranges from -4 to 1 with a step of 1 . In the case of the CNN classifier, a cross-validation procedure over the parameters of this model can be intractable. Thus, in this case we have considered $8 \mathrm{CNN}$ configurations (see Table B.1) varying the number of feature maps in each convolutional layer, the size of the convolution kernels, and the number of channels of the images introduced to the network (i.e., grayscale or RGB images).

All the CNN configurations have been trained using the Keras library with TensorFlow as backend. The CNN models evaluated here consist of 6-layer CNNs: two convolutional layers, two $2 \times 2$ max-pooling layers with a stride of 2 , and two fully-connected layers with one hidden layer of 256 units and an output layer of 2 units, using ReLU activation function and dropout regularization (Srivastava et al., 2014) in each layer except the final one, in which a softmax activation function was used. The selected loss function was based on the categorical cross-entropy loss, using Adam optimizer for its minimization during a training process of 60 epochs as maximum, and a mini-batch size of 128 images. In addition, the early stopping technique was applied for regularization purposes, taking into account the validation loss with a patience of 5 epochs.

Table B.1: CNN configurations evaluated for bucket/background classification.

\begin{tabular}{ccccccc}
\hline \multirow{2}{*}{ CNN conf. } & Input & \multicolumn{2}{c}{ \#Feature Maps } & \multicolumn{2}{c}{ Kernel size } & \multirow{2}{*}{ dim. } \\
& Conv1 & Conv2 & Conv1 & Conv2 & \#aram (M) \\
\hline CNN1 & $56 \times 72 \times 1$ & 32 & 64 & $3 \times 3$ & $3 \times 3$ & 3.17 \\
CNN2 & $56 \times 72 \times 1$ & 32 & 64 & $5 \times 5$ & $3 \times 3$ & 3.17 \\
CNN3 & $56 \times 72 \times 1$ & 32 & 64 & $5 \times 5$ & $5 \times 5$ & 2.76 \\
CNN4 & $56 \times 72 \times 1$ & 16 & 32 & $5 \times 5$ & $5 \times 5$ & 1.37 \\
CNN5 & $56 \times 72 \times 1$ & 8 & 16 & $5 \times 5$ & $5 \times 5$ & 0.68 \\
CNN6 & $56 \times 72 \times 1$ & 4 & 8 & $5 \times 5$ & $5 \times 5$ & 0.34 \\
CNN7 & $56 \times 72 \times 3$ & 32 & 64 & $3 \times 3 \times 3$ & $3 \times 3$ & 3.17 \\
CNN8 & $56 \times 72 \times 3$ & 32 & 64 & $5 \times 5 \times 3$ & $5 \times 5$ & 2.76 \\
\hline
\end{tabular}

The results obtained after conducting the 6 evaluation tests on each classifier are shown in Fig. B.2a and B.2b and summarized in Table B.2 and B.3, where C1 and C2 stand for Class 1 (bucket class) and Class 2 (background class) respectively. In these tables, the average values over the 6 tests are presented. In Table B.2, the high scores obtained in most of the CNN configurations for the training set can be noticed. More relevant are the results presented in Table B.3 for the test sets, where all the CNN configurations obtained a significantly higher $F_{1}$ score than the one obtained by the L2R-LR and LSVM classifiers. This fact can be confirmed in Fig. B.2b, where in all the evaluation tests the CNN-based classifiers obtained a higher performance than the L2R-LR and L-SVM. As shown in Table B.3, the number of feature maps was confirmed as being a crucial parameter for the CNN-based classifiers, where CNN6 achieved a significantly lower performance as compared to the rest of CNN configurations. Another important result derived from Fig. B.2b and Table B.3 is that CNN configurations with a convolution kernel of size $5 \times 5$ provided higher performance than the analogous configurations with 
a kernel size of $3 \times 3$ (i.e., CNN3 vs CNN1, and CNN8 vs CNN7). However, these CNN configurations (CNN3 and CNN8) require the highest processing times per image and are quite demanding in terms of the number of parameters, which will also lead to a higher memory consumption. All the results presented in Table B.2 and B.3 have been obtained with a CPU Intel Core i7-6700HQ except the test time presented in Table B.3, which has been measured with the UAV's onboard computer (Intel NUC6i5SYK featuring a 2.9 $\mathrm{GHz}$ Intel Core i5-6260U CPU), and refers to the processing time per image, averaged over 128 images, taking into account the feature extraction plus the inference time of the classifier. Thus, in the case of the L2R-LR and the L-SVM, the test time encompasses the HOG feature extraction process plus the inference time of the classifier.

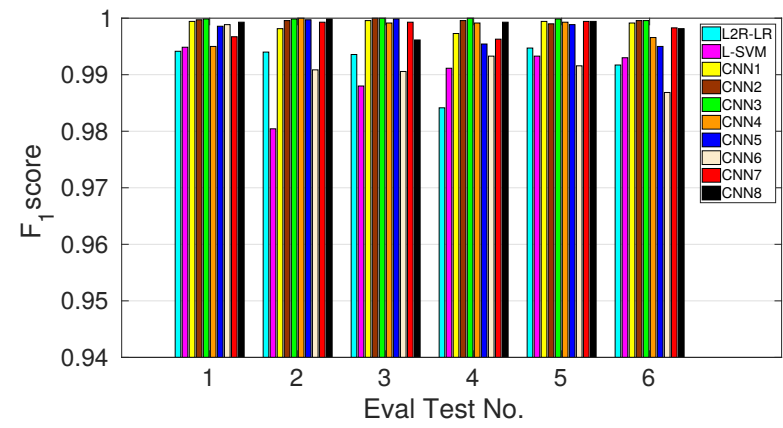

(a)

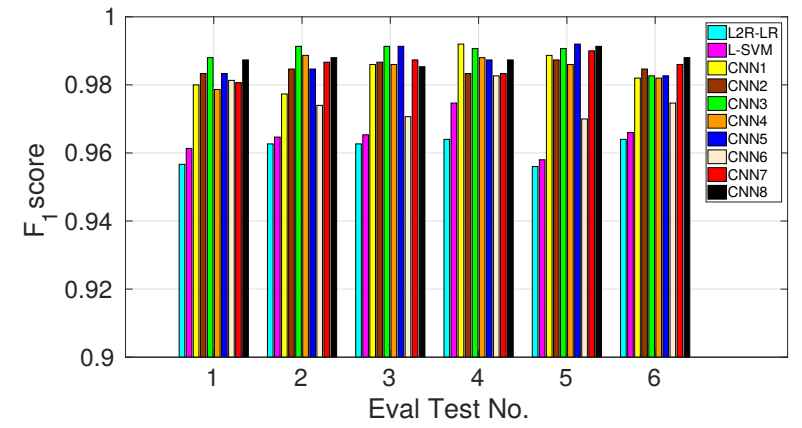

(b)

Figure B.2: Results obtained in the comparison of the supervised learning classifiers used for bucket/background classification on 6 different evaluation tests (best seen in color). (a) $F_{1}$ score obtained in the training sets. (b) $F_{1}$ score obtained in the test sets.

Table B.2: Average training results obtained for the 6 evaluation tests of Fig. B.2a. Final selected model is indicated in bold.

\begin{tabular}{cccccccc}
\hline \multirow{2}{*}{ Classifier } & \multicolumn{2}{c}{ Precision } & \multicolumn{2}{c}{ Recall } & \multicolumn{2}{c}{$F_{1}$ score } & Train time \\
& $\mathrm{C} 1$ & $\mathrm{C} 2$ & $\mathrm{C} 1$ & $\mathrm{C} 2$ & $\mathrm{C} 1$ & $\mathrm{C} 2$ & (s/epoch) \\
\hline CNN1 & 0.998 & 1.00 & 1.00 & 0.998 & 0.999 & 0.999 & 30 \\
CNN2 & 0.999 & 1.00 & 1.00 & 0.999 & 1.00 & 1.00 & 31 \\
CNN3 & 1.00 & 1.00 & 1.00 & 1.00 & 1.00 & 1.00 & 44 \\
CNN4 & 0.997 & 1.00 & 1.00 & 0.997 & 0.998 & 0.998 & 20 \\
CNN5 & $\mathbf{0 . 9 9 6}$ & $\mathbf{1 . 0 0}$ & $\mathbf{1 . 0 0}$ & $\mathbf{0 . 9 9 6}$ & $\mathbf{0 . 9 9 8}$ & $\mathbf{0 . 9 9 8}$ & $\mathbf{1 0}$ \\
CNN6 & 0.987 & 0.997 & 0.997 & 0.987 & 0.992 & 0.992 & 7 \\
CNN7 & 0.996 & 1.00 & 1.00 & 0.996 & 0.998 & 0.998 & 33 \\
CNN8 & 0.998 & 1.00 & 1.00 & 0.998 & 0.999 & 0.999 & 46 \\
L2R-LR & 0.992 & 0.992 & 0.992 & 0.992 & 0.992 & 0.992 & - \\
L-SVM & 0.989 & 0.991 & 0.991 & 0.989 & 0.990 & 0.990 & - \\
\hline
\end{tabular}


Table B.3: Average test results obtained for the 6 evaluation tests of Fig. B.2b. Final selected model is indicated in bold.

\begin{tabular}{cccccccc}
\hline \multirow{2}{*}{ Classifier } & \multicolumn{2}{c}{ Precision } & \multicolumn{2}{c}{ Recall } & \multicolumn{2}{c}{$F_{1}$ score } & Test time \\
& $\mathrm{C} 1$ & $\mathrm{C} 2$ & $\mathrm{C} 1$ & $\mathrm{C} 2$ & $\mathrm{C} 1$ & $\mathrm{C} 2$ & (ms/image) \\
\hline CNN1 & 0.973 & 0.996 & 0.996 & 0.973 & 0.985 & 0.984 & 3.830 \\
CNN2 & 0.978 & 0.993 & 0.993 & 0.977 & 0.985 & 0.985 & 3.956 \\
CNN3 & 0.985 & 0.993 & 0.993 & 0.985 & 0.989 & 0.989 & 5.136 \\
CNN4 & 0.973 & 0.998 & 0.998 & 0.972 & 0.985 & 0.985 & 3.027 \\
CNN5 & $\mathbf{0 . 9 7 8}$ & $\mathbf{0 . 9 9 6}$ & $\mathbf{0 . 9 9 6}$ & $\mathbf{0 . 9 7 8}$ & $\mathbf{0 . 9 8 7}$ & $\mathbf{0 . 9 8 7}$ & $\mathbf{2 . 0 7 5}$ \\
CNN6 & 0.963 & 0.989 & 0.989 & 0.962 & 0.976 & 0.975 & 1.921 \\
CNN7 & 0.974 & 0.998 & 0.998 & 0.973 & 0.986 & 0.985 & 4.123 \\
CNN8 & 0.980 & 0.996 & 0.996 & 0.980 & 0.988 & 0.988 & 5.830 \\
L2R-LR & 0.958 & 0.965 & 0.965 & 0.957 & 0.961 & 0.961 & 0.984 \\
L-SVM & 0.966 & 0.964 & 0.964 & 0.966 & 0.965 & 0.965 & 4.187 \\
\hline
\end{tabular}

The execution of SAR missions using a fully-autonomous aerial robot implies running simultaneously multiple processes that implement the functionalities of each component of the system. Traditionally, UAVs are equipped with small and light computers with limited computational capabilities. In order to deal with these computational constraints, it is required to select the appropriate model in order to find a proper trade-off between performance and computational cost. For this purpose, in this work, we consider the selection of the most appropriate classifier based on two main variables: the performance of the classifier and the processing time. Thus, we take into consideration the average $F_{1}$ score and the test time presented in Table B.3, which are plotted in Fig. B.3 for a better visualization. Taking into account the results presented in Fig. B.3, and considering that the best possible classifier is the one lying on point $(0,1)$ (i.e., minimizing the processing time while maximizing the $F_{1}$ score), CNN5 is selected as the most appropriate classifier for our purposes. This configuration provides the lowest Euclidean distance (in normalized coordinates) to the desired point.

In Fig. B.4 and B.5 the detection of the targets in different time instants during the execution of the third real-flight SAR mission presented in Section 6.7.3 is depicted. It is important to remark the capability of our system to automatically switch between different detection modes. Based on this, once the object is considered as detected using the front camera image, the system stops processing the images coming from that device and starts processing the images coming from the bottom camera. Subsequently, when the release maneuver for the rescue operation is finished, the detection mode using the front camera is enabled again, stopping the processing with the bottom device. 


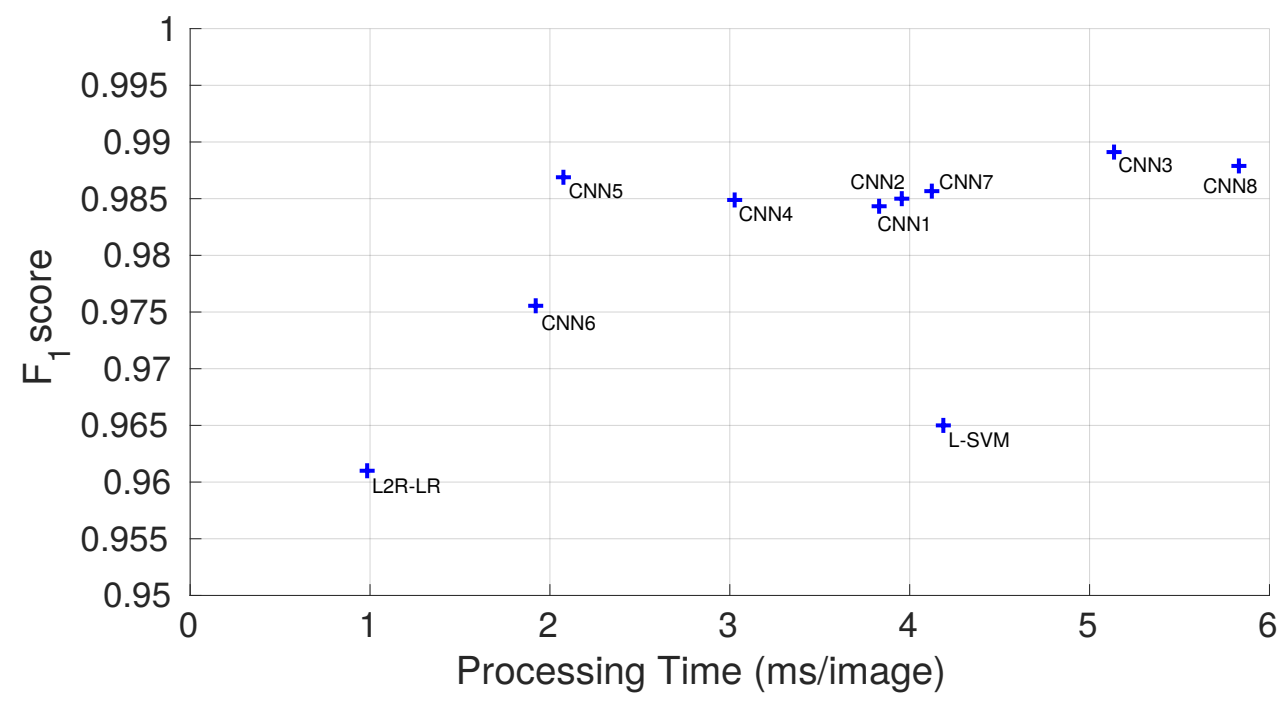

Figure B.3: Average $F_{1}$ score and processing time for the different supervised learning classifiers from the results presented in Table B.3. The processing time has been measured on an Intel NUC6i5SYK computer. 


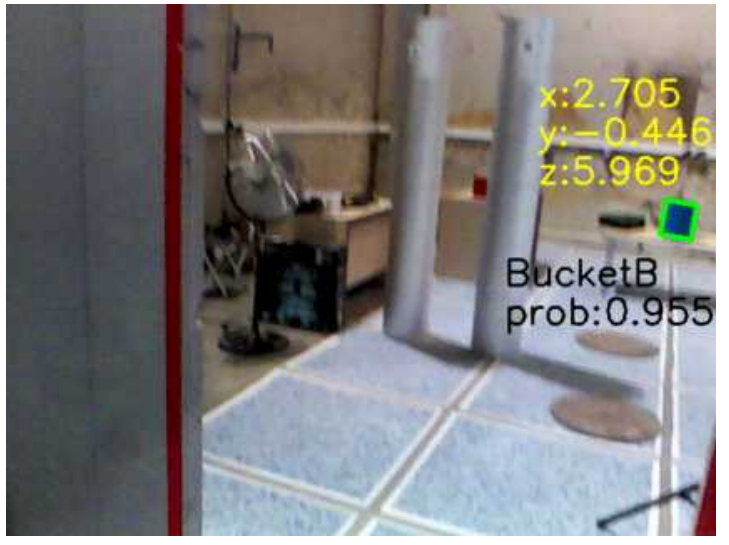

(a)

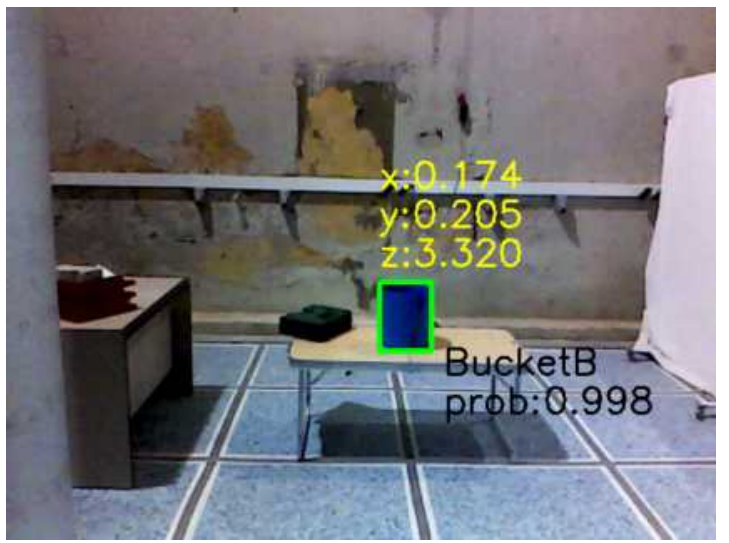

(c)

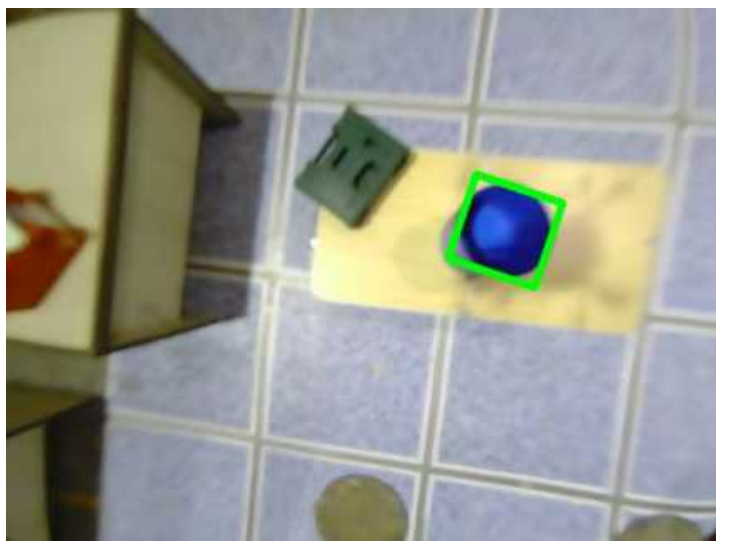

(e)

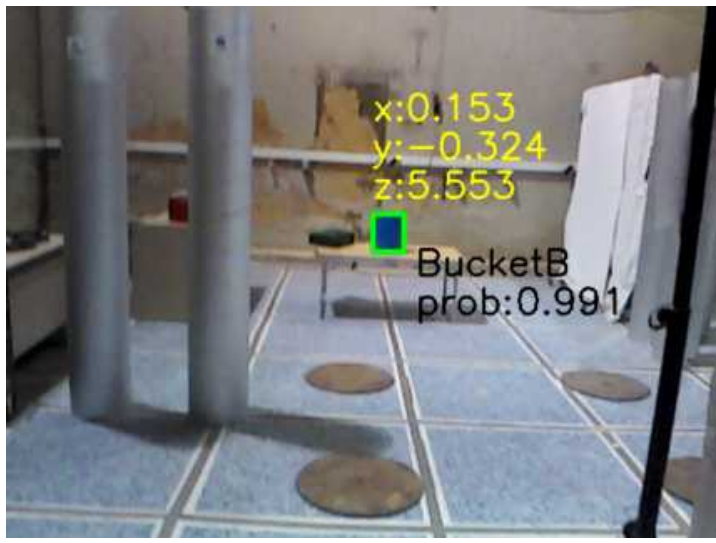

(b)

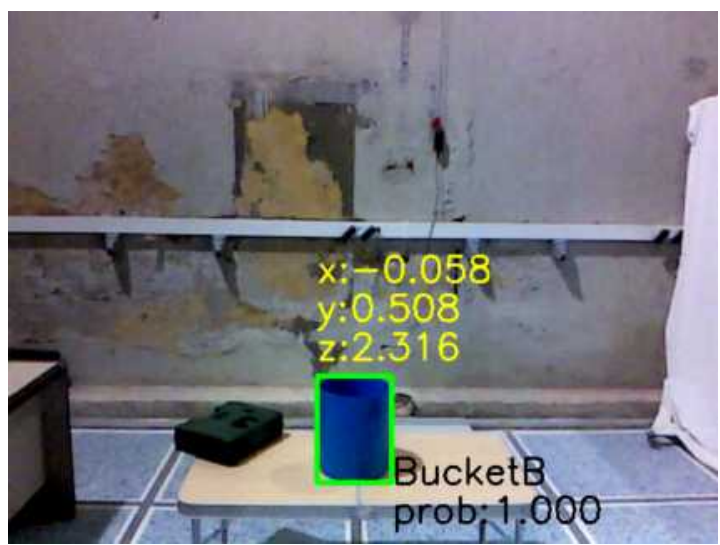

(d)

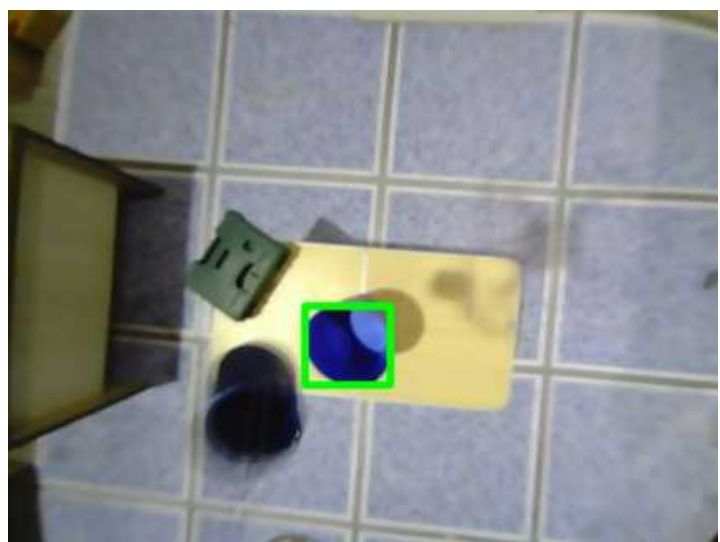

(f)

Figure B.4: Object detection results for target 1 in the third real-flight experiment of Section 6.7.3 (best seen in color). (a) to (d) Detection of the blue bucket (bucket B) from the front camera. (e), (f) Detection of the bucket from the bottom camera. (f) Detection of the bucket in the moment when the release command has just been triggered. The positions indicated in the figure refer to the computed relative pose of the target with respect to the front camera using a $\mathrm{P} n \mathrm{P}$ algorithm. The output probabilities from the CNN5 classifier are indicated together with the object category. 


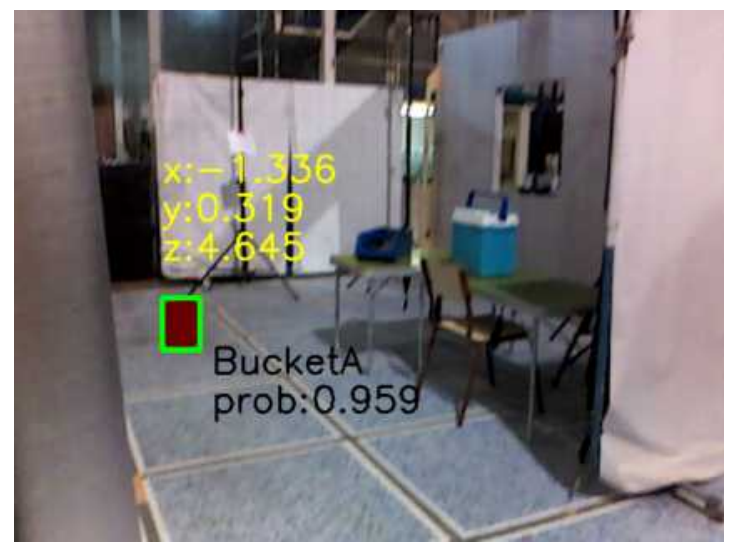

(a)

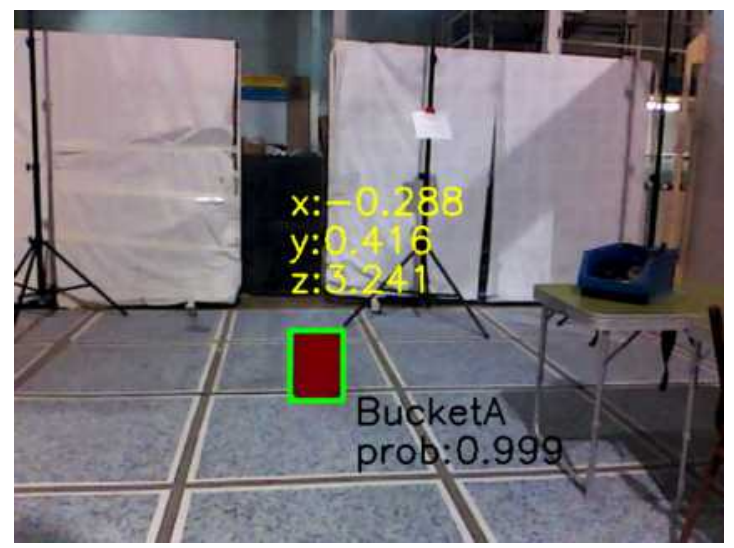

(c)

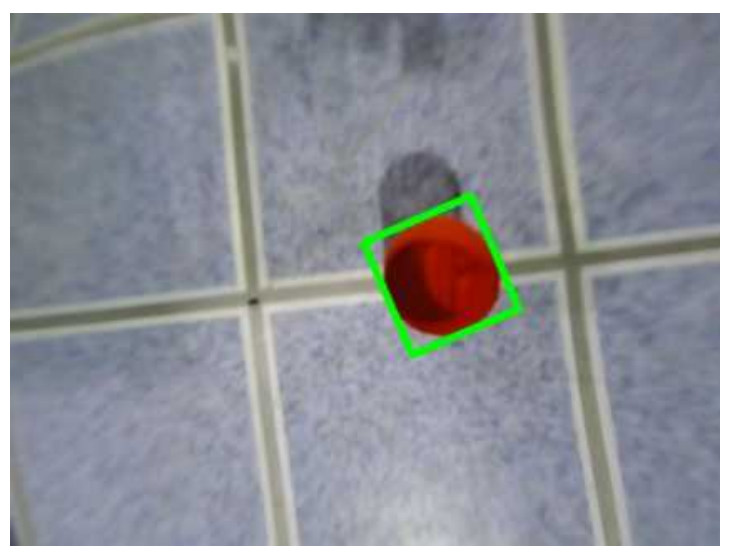

(e)

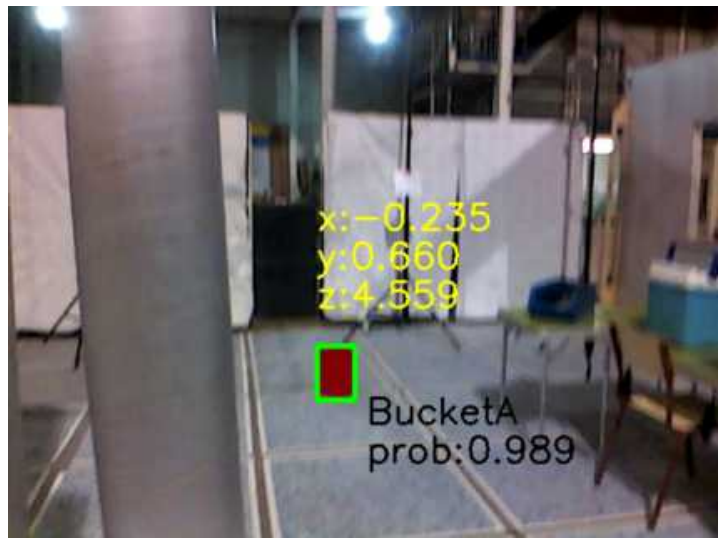

(b)

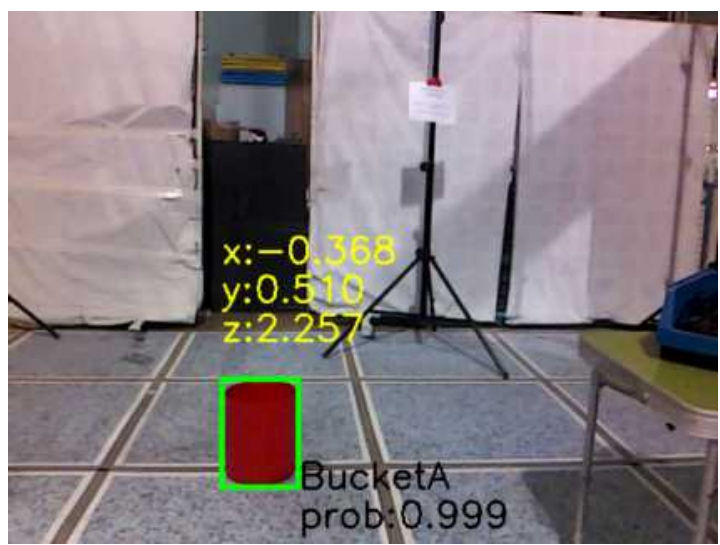

(d)

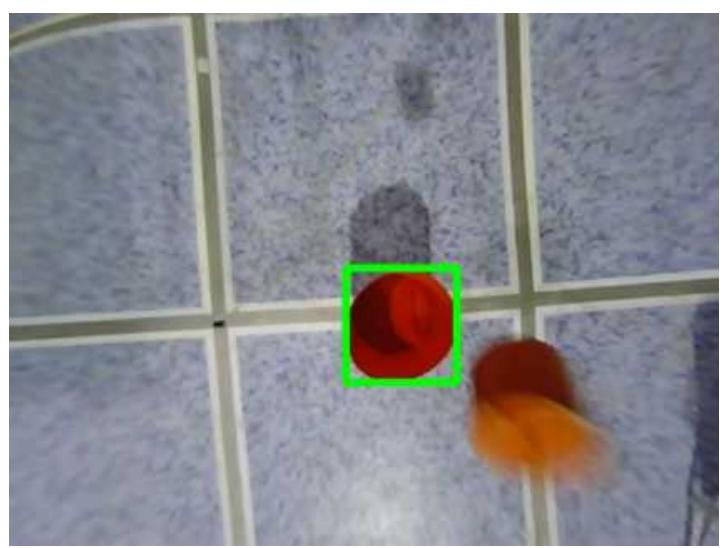

(f)

Figure B.5: Object detection results for target 2 in the third real-flight experiment of Section 6.7.3 (best seen in color). (a) to (d) Detection of the red bucket (bucket A) from the front camera. (e), (f) Detection of the bucket from the bottom camera. (f) Detection of the bucket in the moment when the release command has just been triggered. The positions indicated in the figure refer to the computed relative pose of the target with respect to the front camera using a $\mathrm{P} n \mathrm{P}$ algorithm. The output probabilities from the CNN5 classifier are indicated together with the object category. 


\section{Appendix \\ Scientific Dissemination}

This appendix presents the most relevant publications in which the author of this thesis has actively participated during the period of stay in the Computer Vision \& Aerial Robotics group:

\section{C.1 Journal Publications}

- Carrio, A., Sampedro, C., Rodriguez-Ramos, A., and Campoy, P. (2017). A review of deep learning methods and applications for unmanned aerial vehicles. Journal of Sensors, 2017

- Sanchez-Lopez, J. L., Molina, M., Bavle, H., Sampedro, C., Fernández, R. A. S., and Campoy, P. (2017a). A multi-layered component-based approach for the development of aerial robotic systems: The aerostack framework. Journal of Intelligent E Robotic Systems, 88(2-4):683-709

- Martinez, C., Sampedro, C., Chauhan, A., Collumeau, J. F., and Campoy, P. (2018). The power line inspection software (polis): A versatile system for automating power line inspection. Engineering Applications of Artificial Intelligence, 71:293-314

- Adapted by permission from Springer Nature: Sampedro, C., Rodriguez-Ramos, A., Bavle, H., Carrio, A., de la Puente, P., and Campoy, P. (2018b). A fullyautonomous aerial robot for search and rescue applications in indoor environments using learning-based techniques. Journal of Intelligent $\&$ Robotic Systems, pages $1-27$ (C)(2018)

- (CIEEE. Adapted, with permission from: Sampedro, C., Rodriguez-Vazquez, J., Rodriguez-Ramos, A., Carrio, A., and Campoy, P. (2019). Deep learning-based system for automatic recognition and diagnosis of electrical insulator strings. IEEE Access, 7:101283-101308

- Rodriguez-Ramos, A., Sampedro, C., Bavle, H., De La Puente, P., and Campoy, P. (2019). A deep reinforcement learning strategy for uav autonomous landing on a moving platform. Journal of Intelligent $\&$ Robotic Systems, 93(1-2):351-366 


\section{C.2 Publications in International Peer-Reviewed Con- ferences}

- Sampedro, C., Martinez, C., Chauhan, A., and Campoy, P. (2014). A supervised approach to electric tower detection and classification for power line inspection. In 2014 International Joint Conference on Neural Networks (IJCNN), pages 19701977. IEEE

- Martinez, C., Sampedro, C., Chauhan, A., and Campoy, P. (2014). Towards autonomous detection and tracking of electric towers for aerial power line inspection. In 2014 International Conference on Unmanned Aircraft Systems (ICUAS), pages 284-295. IEEE

- Sampedro, C., Bavle, H., Sanchez-Lopez, J. L., Fernández, R. A. S., RodríguezRamos, A., Molina, M., and Campoy, P. (2016). A flexible and dynamic mission planning architecture for uav swarm coordination. In 2016 International Conference on Unmanned Aircraft Systems (ICUAS), pages 355-363. IEEE

- Carrio, A., Sampedro, C., Fu, C., Collumeau, J.-F., and Campoy, P. (2016). A real-time supervised learning approach for sky segmentation onboard unmanned aerial vehicles. In 2016 International Conference on Unmanned Aircraft Systems (ICUAS), pages 8-14. IEEE

- Sanchez-Lopez, J. L., Fernández, R. A. S., Bavle, H., Sampedro, C., Molina, M., Pestana, J., and Campoy, P. (2016). Aerostack: An architecture and open-source software framework for aerial robotics. In Unmanned Aircraft Systems (ICUAS), 2016 International Conference on, pages 332-341. IEEE

- Fernandez, R. A. S., Sanchez-Lopez, J. L., Sampedro, C., Bavle, H., Molina, M., and Campoy, P. (2016). Natural user interfaces for human-drone multi-modal interaction. In 2016 International Conference on Unmanned Aircraft Systems (ICUAS), pages 1013-1022. IEEE

- Sampedro, C., Bavle, H., Rodríguez-Ramos, A., Carrio, A., Fernández, R. A. S., Sanchez-Lopez, J. L., and Campoy, P. (2017). A fully-autonomous aerial robotic solution for the 2016 international micro air vehicle competition. In 2017 International conference on unmanned aircraft systems (ICUAS), pages 989-998. IEEE

- Rodriguez-Ramos, A., Sampedro, C., Bavle, H., Milosevic, Z., Garcia-Vaquero, A., and Campoy, P. (2017). Towards fully autonomous landing on moving platforms for rotary unmanned aerial vehicles. In 2017 International Conference on Unmanned Aircraft Systems (ICUAS), pages 170-178. IEEE

- Sampedro, C., Bavle, H., Rodriguez-Ramos, A., de la Puente, P., and Campoy, P. (2018a). Laser-based reactive navigation for multirotor aerial robots using deep reinforcement learning. In 2018 IEEE/RSJ International Conference on Intelligent Robots and Systems (IROS), pages 1024-1031. IEEE 
- Sampedro, C., Rodriguez-Ramos, A., Gil, I., Mejias, L., and Campoy, P. (2018c). Image-based visual servoing controller for multirotor aerial robots using deep reinforcement learning. In 2018 IEEE/RSJ International Conference on Intelligent Robots and Systems (IROS), pages 979-986. IEEE

- Rodriguez-Ramos, A., Sampedro, C., Bavle, H., Moreno, I. G., and Campoy, P. (2018). A deep reinforcement learning technique for vision-based autonomous multirotor landing on a moving platform. In 2018 IEEE/RSJ International Conference on Intelligent Robots and Systems (IROS), pages 1010-1017

- Lu, L., Sampedro, C., Rodriguez-Vazquez, J., and Campoy, P. (2019). Laser-based collision avoidance and reactive navigation using rrt* $^{*}$ and signed distance field for multirotor uavs. In 2019 International Conference on Unmanned Aircraft Systems (ICUAS), pages 1209-1217

- Sanchez-Lopez, J. L., Sampedro, C., Cazzato, D., and Voos, H. (2019). Deep learning based semantic situation awareness system for multirotor aerial robots using lidar. In 2019 International Conference on Unmanned Aircraft Systems (ICUAS), pages 899-908

\section{C.3 Digital Media}

All the videos shown in the corresponding Experiments and Results sections within each chapter can be additionally reviewed in the following video-sharing websites of the Computer Vision \& Aerial Robotics group (formerly called Vision4UAV):

- Vimeo Vision4UAV channel: https://vimeo.com/vision4uav

- Youtube Vision4UAV channel: https://www.youtube.com/channel/UCfry6dL6G5Sd3U49LC9wvYQ 



\section{Bibliography}

Abbeel, P., Coates, A., Quigley, M., and Ng, A. Y. (2007). An application of reinforcement learning to aerobatic helicopter flight. In Advances in neural information processing systems, pages 1-8.

Abe, N., Zadrozny, B., and Langford, J. (2006). Outlier detection by active learning. In Proceedings of the 12th ACM SIGKDD international conference on Knowledge discovery and data mining, pages 504-509. ACM.

Adams, S. M. and Friedland, C. J. (2011). A survey of unmanned aerial vehicle (UAV) usage for imagery collection in disaster research and management. publisher not identified.

Adelson, E. H., Anderson, C. H., Bergen, J. R., Burt, P. J., and Ogden, J. M. (1984). Pyramid methods in image processing. RCA engineer, 29(6):33-41.

Alaverdyan, Z., Chai, J., and Lartizien, C. (2018). Unsupervised feature learning for outlier detection with stacked convolutional autoencoders, siamese networks and wasserstein autoencoders: Application to epilepsy detection. In Deep Learning in Medical Image Analysis and Multimodal Learning for Clinical Decision Support, pages 210217. Springer.

Alexe, B., Deselaers, T., and Ferrari, V. (2010). What is an object? In 2010 IEEE Computer Society Conference on Computer Vision and Pattern Recognition, pages 73-80. IEEE.

Alfeo, A. L., Cimino, M. G., De Francesco, N., Lazzeri, A., Lega, M., and Vaglini, G. (2018). Swarm coordination of mini-uavs for target search using imperfect sensors. Intelligent Decision Technologies, 12(2):149-162.

Araar, O. and Aouf, N. (2014). Visual servoing of a quadrotor uav for autonomous power lines inspection. In Control and Automation (MED), 2014 22nd Mediterranean Conference of, pages 1418-1424. IEEE.

Azizpour, H. and Laptev, I. (2012). Object detection using strongly-supervised deformable part models. In European Conference on Computer Vision, pages 836-849. Springer.

Badrinarayanan, V., Kendall, A., and Cipolla, R. (2017). Segnet: A deep convolutional encoder-decoder architecture for image segmentation. IEEE transactions on pattern analysis and machine intelligence, 39(12):2481-2495. 
Bahill, A. T. and Gissing, B. (1998). Re-evaluating systems engineering concepts using systems thinking. IEEE Transactions on Systems, Man, and Cybernetics, Part C (Applications and Reviews), 28(4):516-527.

Bateux, Q., Marchand, E., Leitner, J., Chaumette, F., and Corke, P. (2017). Visual servoing from deep neural networks. arXiv preprint arXiv:1705.08940.

Bavle, H., Sanchez-Lopez, J. L., Rodriguez-Ramos, A., Sampedro, C., and Campoy, P. (2017). A flight altitude estimator for multirotor uavs in dynamic and unstructured indoor environments. In 2017 International Conference on Unmanned Aircraft Systems (ICUAS), pages 1044-1051.

Bejiga, M. B., Zeggada, A., Nouffidj, A., and Melgani, F. (2017). A convolutional neural network approach for assisting avalanche search and rescue operations with uav imagery. Remote Sensing, 9(2):100.

Bishop, C. M. (2006). Pattern recognition and machine learning. springer.

Boser, B. E., Guyon, I. M., and Vapnik, V. N. (1992). A training algorithm for optimal margin classifiers. In Proceedings of the fifth annual workshop on Computational learning theory, pages 144-152. ACM.

Boškovic, J. D., Knoebel, N., Moshtagh, N., Amin, J., and Larson, G. L. (2009). Collaborative mission planning \& autonomous control technology (compact) system employing swarms of uavs. In AIAA Guidance, Navigation, and Control Conference, Chicago.

Brockman, G., Cheung, V., Pettersson, L., Schneider, J., Schulman, J., Tang, J., and Zaremba, W. (2016). Openai gym. arXiv preprint arXiv:1606.01540.

Buitrago, N. R. S., Tonnaer, L., Menkovski, V., and Mavroeidis, D. (2018). Anomaly detection for imbalanced datasets with deep generative models. CoRR, abs/1811.00986.

Canny, J. (1987). A computational approach to edge detection. In Readings in computer vision, pages 184-203. Elsevier.

Carrio, A., Sampedro, C., Fu, C., Collumeau, J.-F., and Campoy, P. (2016). A realtime supervised learning approach for sky segmentation onboard unmanned aerial vehicles. In 2016 International Conference on Unmanned Aircraft Systems (ICUAS), pages 8-14. IEEE.

Carrio, A., Sampedro, C., Rodriguez-Ramos, A., and Campoy, P. (2017). A review of deep learning methods and applications for unmanned aerial vehicles. Journal of Sensors, 2017.

Caruana, R. and Niculescu-Mizil, A. (2006). An empirical comparison of supervised learning algorithms. In Proceedings of the 23rd international conference on Machine learning, pages 161-168. ACM. 
Chandola, V., Banerjee, A., and Kumar, V. (2009). Anomaly detection: A survey. ACM computing surveys (CSUR), 41(3):15.

Chang, C.-C. and Lin, C.-J. (2011). Libsvm: A library for support vector machines. ACM transactions on intelligent systems and technology (TIST), 2(3):27.

Chang, W., Yang, G., Yu, J., and Liang, Z. (2018). Real-time segmentation of various insulators using generative adversarial networks. IET Computer Vision, 12(5):596602.

Chaumette, F. (1998). Potential problems of stability and convergence in image-based and position-based visual servoing. In The confluence of vision and control, pages 66-78. Springer.

Chaumette, F. and Hutchinson, S. (2006a). Visual servo control. i. basic approaches. IEEE Robotics Automation Magazine, 13(4):82-90.

Chaumette, F. and Hutchinson, S. (2006b). Visual servo control, part i: Basic approaches. IEEE Robotics $\&$ Automation Magazine, 13(4):82-90.

Chaumette, F. and Hutchinson, S. (2007). Visual servo control, part ii: Advanced approaches. IEEE Robotics and Automation Magazine, 14(1):109-118.

Chen, J., Liu, T., and Shen, S. (2016). Online generation of collision-free trajectories for quadrotor flight in unknown cluttered environments. In Robotics and Automation (ICRA), 2016 IEEE International Conference on, pages 1476-1483. IEEE.

Cheng, M.-M., Zhang, Z., Lin, W.-Y., and Torr, P. (2014). Bing: Binarized normed gradients for objectness estimation at 300fps. In Proceedings of the IEEE conference on computer vision and pattern recognition, pages 3286-3293.

Choi, S., Kim, S., and Kim, H. J. (2017). Inverse reinforcement learning control for trajectory tracking of a multirotor uav. International Journal of Control, Automation and Systems, 15(4):1826-1834.

Chopra, S., Hadsell, R., and LeCun, Y. (2005). Learning a similarity metric discriminatively, with application to face verification. In 2005 IEEE Computer Society Conference on Computer Vision and Pattern Recognition (CVPR'05), volume 1, pages 539-546 vol. 1.

Corke, P. I. and Hutchinson, S. A. (2001). A new partitioned approach to image-based visual servo control. IEEE Transactions on Robotics and Automation, 17(4):507-515.

Cui, J. Q., Phang, S. K., Ang, K. Z., Wang, F., Dong, X., Ke, Y., Lai, S., Li, K., Li, X., Lin, J., et al. (2016). Search and rescue using multiple drones in post-disaster situation. Unmanned Systems, 4(01):83-96. 
Dalal, N. and Triggs, B. (2005). Histograms of oriented gradients for human detection. In 2005 IEEE Computer Society Conference on Computer Vision and Pattern Recognition (CVPR'05), volume 1, pages 886-893 vol. 1.

Dasgupta, P. (2008). A multiagent swarming system for distributed automatic target recognition using unmanned aerial vehicles. Systems, Man and Cybernetics, Part A: Systems and Humans, IEEE Transactions on, 38(3):549-563.

Deng, C., Wang, S., Huang, Z., Tan, Z., and Liu, J. (2014). Unmanned aerial vehicles for power line inspection: A cooperative way in platforms and communications. $J$. Commun, 9(9):687-692.

Deng, J., Dong, W., Socher, R., Li, L.-J., Li, K., and Fei-Fei, L. (2009). Imagenet: A large-scale hierarchical image database. In 2009 IEEE conference on computer vision and pattern recognition, pages 248-255. IEEE.

Doherty, P. and Rudol, P. (2007). A uav search and rescue scenario with human body detection and geolocalization. In Australian Conference on Artificial Intelligence, volume 4830, pages 1-13. Springer.

Dorigo, M. and Colombetti, M. (1998). Robot shaping: an experiment in behavior engineering. MIT press.

Duchi, J., Hazan, E., and Singer, Y. (2011). Adaptive subgradient methods for online learning and stochastic optimization. Journal of Machine Learning Research, 12(Jul):2121-2159.

Erez, T. and Smart, W. D. (2008). What does shaping mean for computational reinforcement learning? In Development and Learning, 2008. ICDL 2008. 7th IEEE International Conference on, pages 215-219. IEEE.

Erfani, S. M., Rajasegarar, S., Karunasekera, S., and Leckie, C. (2016). High-dimensional and large-scale anomaly detection using a linear one-class svm with deep learning. Pattern Recognition, 58:121-134.

Espiau, B. (1994). Effect of camera calibration errors on visual servoing in robotics. In Experimental robotics III, pages 182-192. Springer.

Fan, R.-E., Chang, K.-W., Hsieh, C.-J., Wang, X.-R., and Lin, C.-J. (2008). Liblinear: A library for large linear classification. Journal of machine learning research, 9(Aug):1871-1874.

Felzenszwalb, P. F., Girshick, R. B., McAllester, D., and Ramanan, D. (2009). Object detection with discriminatively trained part-based models. IEEE transactions on pattern analysis and machine intelligence, 32(9):1627-1645. 
Fernandez, R. A. S., Sanchez-Lopez, J. L., Sampedro, C., Bavle, H., Molina, M., and Campoy, P. (2016). Natural user interfaces for human-drone multi-modal interaction. In 2016 International Conference on Unmanned Aircraft Systems (ICUAS), pages 1013-1022. IEEE.

Forsberg, K. and Mooz, H. (1991). The relationship of system engineering to the project cycle. In INCOSE International Symposium, volume 1, pages 57-65. Wiley Online Library.

Fukushima, K. (1980). Neocognitron: A self-organizing neural network model for a mechanism of pattern recognition unaffected by shift in position. Biological cybernetics, 36(4):193-202.

Furrer, F., Burri, M., Achtelik, M., and Siegwart, R. (2016). Rotors-a modular gazebo mav simulator framework. In Robot Operating System (ROS), pages 595-625. Springer.

Gancet, J., Hattenberger, G., Alami, R., and Lacroix, S. (2005). Task planning and control for a multi-uav system: architecture and algorithms. In Intelligent Robots and Systems, 2005.(IROS 2005). 2005 IEEE/RSJ International Conference on, pages 1017-1022. IEEE.

Gandhi, D., Pinto, L., and Gupta, A. (2017). Learning to fly by crashing. In 2017 IEEE/RSJ International Conference on Intelligent Robots and Systems (IROS), pages 3948-3955.

Gao, F., Wang, J., Kong, Z., Wu, J., Feng, N., Wang, S., Hu, P., Li, Z., Huang, H., and Li, J. (2017). Recognition of insulator explosion based on deep learning. In 2017 14th International Computer Conference on Wavelet Active Media Technology and Information Processing (ICCWAMTIP), pages 79-82. IEEE.

Garrido-Jurado, S., Muñoz-Salinas, R., Madrid-Cuevas, F. J., and Marín-Jiménez, M. J. (2014). Automatic generation and detection of highly reliable fiducial markers under occlusion. Pattern Recognition, 47(6):2280-2292.

Gaudiano, P., Bonabeau, E., and Shargel, B. (2005). Evolving behaviors for a swarm of unmanned air vehicles. In Swarm Intelligence Symposium, 2005. SIS 2005. Proceedings 2005 IEEE, pages 317-324. IEEE.

Girshick, R. (2015). Fast r-cnn. In Proceedings of the IEEE International Conference on Computer Vision, pages 1440-1448.

Girshick, R., Donahue, J., Darrell, T., and Malik, J. (2014). Rich feature hierarchies for accurate object detection and semantic segmentation. In Proceedings of the IEEE conference on computer vision and pattern recognition, pages 580-587.

Glorot, X., Bordes, A., and Bengio, Y. (2011). Deep sparse rectifier neural networks. In Proceedings of the fourteenth international conference on artificial intelligence and statistics, pages 315-323. 
Goodfellow, I., Bengio, Y., and Courville, A. (2016). Deep learning. MIT press.

Goodfellow, I., Pouget-Abadie, J., Mirza, M., Xu, B., Warde-Farley, D., Ozair, S., Courville, A., and Bengio, Y. (2014). Generative adversarial nets. In Advances in neural information processing systems, pages 2672-2680.

Gu, S., Lillicrap, T., Sutskever, I., and Levine, S. (2016). Continuous deep q-learning with model-based acceleration. In International Conference on Machine Learning, pages 2829-2838.

Gubbi, J., Varghese, A., and Balamuralidhar, P. (2017). A new deep learning architecture for detection of long linear infrastructure. In 2017 Fifteenth IAPR International Conference on Machine Vision Applications (MVA), pages 207-210. IEEE.

Han, Y., Liu, Z., Lee, D., Liu, W., Chen, J., and Han, Z. (2018). Computer visionbased automatic rod-insulator defect detection in high-speed railway catenary system. International Journal of Advanced Robotic Systems, 15(3):1729881418773943.

He, K., Zhang, X., Ren, S., and Sun, J. (2015a). Delving deep into rectifiers: Surpassing human-level performance on imagenet classification. In Proceedings of the IEEE international conference on computer vision, pages 1026-1034.

He, K., Zhang, X., Ren, S., and Sun, J. (2015b). Spatial pyramid pooling in deep convolutional networks for visual recognition. IEEE transactions on pattern analysis and machine intelligence, 37(9):1904-1916.

He, K., Zhang, X., Ren, S., and Sun, J. (2016). Deep residual learning for image recognition. In Proceedings of the IEEE conference on computer vision and pattern recognition, pages 770-778.

Hwangbo, J., Sa, I., Siegwart, R., and Hutter, M. (2017). Control of a quadrotor with reinforcement learning. IEEE Robotics and Automation Letters, 2(4):2096-2103.

Imanberdiyev, N., Fu, C., Kayacan, E., and Chen, I.-M. (2016). Autonomous navigation of uav by using real-time model-based reinforcement learning. In 201614 th International Conference on Control, Automation, Robotics and Vision (ICARCV), pages 1-6. IEEE.

Ioffe, S. and Szegedy, C. (2015). Batch normalization: Accelerating deep network training by reducing internal covariate shift. arXiv preprint arXiv:1502.0316\%.

Isola, P., Zhu, J.-Y., Zhou, T., and Efros, A. A. (2017). Image-to-image translation with conditional adversarial networks. In Proceedings of the IEEE conference on computer vision and pattern recognition, pages 1125-1134.

Jabid, T. and Ahsan, T. (2018). Insulator detection and defect classification using rotation invariant local directional pattern. International Journal of Advanced Computer Science and Applications, 9(2):265-272. 
Jimenez-Cano, A. E., Sanchez-Cuevas, P. J., Grau, P., Ollero, A., and Heredia, G. (2019). Contact-based bridge inspection multirotors: Design, modeling, and control considering the ceiling effect. IEEE Robotics and Automation Letters, 4(4):3561-3568.

Kahn, G., Villaflor, A., Pong, V., Abbeel, P., and Levine, S. (2017). Uncertainty-aware reinforcement learning for collision avoidance. arXiv preprint arXiv:1702.01182.

Kim, B. and Cho, S. (2018). Automated vision-based detection of cracks on concrete surfaces using a deep learning technique. Sensors, 18(10):3452.

Kingma, D. P. and Ba, J. (2014). Adam: A method for stochastic optimization. arXiv preprint arXiv:1412.6980.

Koch, G., Zemel, R., and Salakhutdinov, R. (2015). Siamese neural networks for one-shot image recognition. In ICML Deep Learning Workshop, volume 2.

Kohlbrecher, S., Von Stryk, O., Meyer, J., and Klingauf, U. (2011). A flexible and scalable slam system with full 3d motion estimation. In 2011 IEEE International Symposium on Safety, Security, and Rescue Robotics, pages 155-160. IEEE.

Koren, Y. and Borenstein, J. (1991). Potential field methods and their inherent limitations for mobile robot navigation. In Proceedings of the IEEE International Conference on Robotics and Automation, pages 1398-1404 vol.2.

Krizhevsky, A., Sutskever, I., and Hinton, G. E. (2012). Imagenet classification with deep convolutional neural networks. In Advances in neural information processing systems, pages 1097-1105.

Kubat, M., Matwin, S., et al. (1997). Addressing the curse of imbalanced training sets: one-sided selection. In Proceedings of the 14 th International Conference on Machine Learning, pages 179-186.

Kumar, V. and Michael, N. (2017). Opportunities and Challenges with Autonomous Micro Aerial Vehicles, pages 41-58. Springer International Publishing.

Lamont, G. B., Slear, J. N., and Melendez, K. (2007). Uav swarm mission planning and routing using multi-objective evolutionary algorithms. In Computational Intelligence in Multicriteria Decision Making, IEEE Symposium on, pages 10-20. IEEE.

Laud, A. D. (2004). Theory and application of reward shaping in reinforcement learning. Technical report.

LaValle, S. M. (2006). Planning algorithms. Cambridge university press.

LeCun, Y., Bottou, L., Bengio, Y., Haffner, P., et al. (1998). Gradient-based learning applied to document recognition. Proceedings of the IEEE, 86(11):2278-2324.

LeCun, Y., Haffner, P., Bottou, L., and Bengio, Y. (1999). Object recognition with gradient-based learning. In Shape, contour and grouping in computer vision, pages 319-345. Springer. 
Lee, A. X., Levine, S., and Abbeel, P. (2017). Learning visual servoing with deep features and fitted q-iteration. arXiv preprint arXiv:1703.11000.

Lee, D., Ryan, T., and Kim, H. J. (2012). Autonomous landing of a vtol uav on a moving platform using image-based visual servoing. In Robotics and Automation (ICRA), 2012 IEEE International Conference on, pages 971-976. IEEE.

Li, S., Liu, T., Zhang, C., Yeung, D.-Y., and Shen, S. (2017). Learning unmanned aerial vehicle control for autonomous target following. arXiv preprint arXiv:1709.08233.

Lillicrap, T. P., Hunt, J. J., Pritzel, A., Heess, N., Erez, T., Tassa, Y., Silver, D., and Wierstra, D. (2015). Continuous control with deep reinforcement learning. arXiv preprint arXiv:1509.02971.

Lin, M., Chen, Q., and Yan, S. (2013). Network in network. arXiv preprint arXiv:1312.4400.

Lin, T.-Y., Dollár, P., Girshick, R., He, K., Hariharan, B., and Belongie, S. (2017). Feature pyramid networks for object detection. In Proceedings of the IEEE Conference on Computer Vision and Pattern Recognition, pages 2117-2125.

Ling, Z., Qiu, R. C., Jin, Z., Zhang, Y., He, X., Liu, H., and Chu, L. (2018). An accurate and real-time self-blast glass insulator location method based on faster $\mathrm{r}$-cnn and u-net with aerial images. arXiv preprint arXiv:1801.05143.

Lippiello, V., Cacace, J., Santamaria-Navarro, A., Andrade-Cetto, J., Trujillo, M. A., Esteves, Y. R., and Viguria, A. (2016). Hybrid visual servoing with hierarchical task composition for aerial manipulation. IEEE Robotics and Automation Letters, $1(1): 259-266$.

Liu, J., Wang, W., Li, X., Wang, T., and Wang, T. (2018). A motif-based mission planning method for uav swarms considering dynamic reconfiguration. Defence Science Journal, 68(2):159-166.

Liu, S., Watterson, M., Tang, S., and Kumar, V. (2016a). High speed navigation for quadrotors with limited onboard sensing. In Robotics and Automation (ICRA), 2016 IEEE International Conference on, pages 1484-1491. IEEE.

Liu, W., Anguelov, D., Erhan, D., Szegedy, C., Reed, S., Fu, C.-Y., and Berg, A. C. (2016b). Ssd: Single shot multibox detector. In European conference on computer vision, pages 21-37. Springer.

Long, J., Shelhamer, E., and Darrell, T. (2015). Fully convolutional networks for semantic segmentation. In Proceedings of the IEEE conference on computer vision and pattern recognition, pages 3431-3440.

Lopez, B. T. and How, J. P. (2017). Aggressive 3-d collision avoidance for high-speed navigation. In 2017 IEEE International Conference on Robotics and Automation (ICRA), pages 5759-5765. 
Lowe, D. G. (2004). Distinctive image features from scale-invariant keypoints. International journal of computer vision, 60(2):91-110.

Lu, L., Sampedro, C., Rodriguez-Vazquez, J., and Campoy, P. (2019). Laser-based collision avoidance and reactive navigation using rrt* and signed distance field for multirotor uavs. In 2019 International Conference on Unmanned Aircraft Systems (ICUAS), pages 1209-1217.

Lumelsky, V. J. and Stepanov, A. A. (1987). Path-planning strategies for a point mobile automaton moving amidst unknown obstacles of arbitrary shape. Algorithmica, $2(1): 403-430$.

Lygouras, E., Santavas, N., Taitzoglou, A., Tarchanidis, K., Mitropoulos, A., and Gasteratos, A. (2019). Unsupervised human detection with an embedded vision system on a fully autonomous uav for search and rescue operations. Sensors, 19(16).

Maas, A. L., Hannun, A. Y., and Ng, A. Y. (2013). Rectifier nonlinearities improve neural network acoustic models. In Proc. icml, volume 30, page 3.

Malis, E. (2004). Improving vision-based control using efficient second-order minimization techniques. In IEEE International Conference on Robotics and Automation, 2004. Proceedings. ICRA'04. 2004, volume 2, pages 1843-1848. IEEE.

Manevitz, L. M. and Yousef, M. (2001). One-class svms for document classification. Journal of machine Learning research, 2(Dec):139-154.

Mao, X., Li, Q., Xie, H., Lau, R. Y., Wang, Z., and Paul Smolley, S. (2017). Least squares generative adversarial networks. In Proceedings of the IEEE International Conference on Computer Vision, pages 2794-2802.

Marchand, É., Spindler, F., and Chaumette, F. (2005). Visp for visual servoing: a generic software platform with a wide class of robot control skills. IEEE Robotics \& A Atomation Magazine, 12(4):40-52.

Marder-Eppstein, E., Berger, E., Foote, T., Gerkey, B., and Konolige, K. (2010). The office marathon: Robust navigation in an indoor office environment. In 2010 IEEE international conference on robotics and automation, pages 300-307. IEEE.

Martin, P., Payton, O., Fardoulis, J., Richards, D., and Scott, T. (2015). Yamashiki, y.(2016). low altitude unmanned aerial vehicle for characterising remediation effectiveness following the fdnpp accident. journal of environmental radioactivity, 153 (1), 58-63. Journal of Environmental Radioactivity, 153(1):58-63.

Martinez, C., Sampedro, C., Chauhan, A., and Campoy, P. (2014). Towards autonomous detection and tracking of electric towers for aerial power line inspection. In 2014 International Conference on Unmanned Aircraft Systems (ICUAS), pages 284-295. IEEE. 
Martinez, C., Sampedro, C., Chauhan, A., Collumeau, J. F., and Campoy, P. (2018). The power line inspection software (polis): A versatile system for automating power line inspection. Engineering Applications of Artificial Intelligence, 71:293-314.

McCune, R. R. and Madey, G. R. (2013). Agent-based simulation of cooperative hunting with uavs. In Proceedings of the Agent-Directed Simulation Symposium, page 8. Society for Computer Simulation International.

McGuire, K., de Croon, G., and Tuyls, K. (2019). A comparative study of bug algorithms for robot navigation. Robotics and Autonomous Systems, page 103261.

Meier, L., Tanskanen, P., Fraundorfer, F., and Pollefeys, M. (2011). Pixhawk: A system for autonomous flight using onboard computer vision. In Robotics and automation (ICRA), 2011 IEEE international conference on, pages 2992-2997. IEEE.

Metni, N. and Hamel, T. (2007). A uav for bridge inspection: Visual servoing control law with orientation limits. Automation in construction, 17(1):3-10.

Miao, X., Liu, X., Chen, J., Zhuang, S., Fan, J., and Jiang, H. (2019). Insulator detection in aerial images for transmission line inspection using single shot multibox detector. IEEE Access, 7:9945-9956.

Michael, N., Shen, S., Mohta, K., Mulgaonkar, Y., Kumar, V., Nagatani, K., Okada, Y., Kiribayashi, S., Otake, K., Yoshida, K., et al. (2012). Collaborative mapping of an earthquake-damaged building via ground and aerial robots. Journal of Field Robotics, 29(5):832-841.

Minguez, J. and Montano, L. (2004). Nearness diagram (ND) navigation: collision avoidance in troublesome scenarios. IEEE Transactions on Robotics and Automation, 20(1):45-59.

Minhas, M. S. and Zelek, J. S. (2019). Anomaly detection in images. CoRR, abs/1905.13147.

Mirowski, P., Pascanu, R., Viola, F., Soyer, H., Ballard, A., Banino, A., Denil, M., Goroshin, R., Sifre, L., Kavukcuoglu, K., et al. (2016). Learning to navigate in complex environments. arXiv preprint arXiv:1611.03673.

Mnih, V., Badia, A. P., Mirza, M., Graves, A., Lillicrap, T., Harley, T., Silver, D., and Kavukcuoglu, K. (2016). Asynchronous methods for deep reinforcement learning. In International Conference on Machine Learning, pages 1928-1937.

Mnih, V., Kavukcuoglu, K., Silver, D., Rusu, A. A., Veness, J., Bellemare, M. G., Graves, A., Riedmiller, M., Fidjeland, A. K., Ostrovski, G., et al. (2015). Human-level control through deep reinforcement learning. Nature, 518(7540):529-533. 
Mondragón, I. F., Campoy, P., Olivares-Mendez, M. A., and Martinez, C. (2011). 3d object following based on visual information for unmanned aerial vehicles. In $I X$ Latin American Robotics Symposium and IEEE Colombian Conference on Automatic Control, 2011 IEEE, pages 1-7. IEEE.

Moore, T. and Stouch, D. (2016). A generalized extended kalman filter implementation for the robot operating system. In Intelligent autonomous systems 13, pages 335-348. Springer.

Mujahed, M., Fischer, D., and Mertsching, B. (2016). Tangential gap flow (TGF) navigation. Robotics and Autonomous Systems, 84(C):15-30.

Muñoz-Marí, J., Bovolo, F., Gómez-Chova, L., Bruzzone, L., and Camp-Valls, G. (2010). Semisupervised one-class support vector machines for classification of remote sensing data. IEEE transactions on geoscience and remote sensing, 48(8):3188-3197.

Muñoz-Marí, J., Gómez-Chova, L., Camps-Valls, G., and Calpe-Maravilla, J. (2008). Image classification with semi-supervised one-class support vector machine. In Image and Signal Processing for Remote Sensing XIV, volume 7109, page 71090B. International Society for Optics and Photonics.

Nair, V. and Hinton, G. E. (2010). Rectified linear units improve restricted boltzmann machines. In Proceedings of the 27th international conference on machine learning (ICML-10), pages 807-814.

Nguyen, G. H., Bouzerdoum, A., and Phung, S. L. (2009). Learning pattern classification tasks with imbalanced data sets. In Pattern recognition. InTech.

Nguyen, M.-N. and Vien, N. A. (2018). Scalable and interpretable one-class svms with deep learning and random fourier features. In Joint European Conference on Machine Learning and Knowledge Discovery in Databases, pages 157-172. Springer.

Nguyen, V. N., Jenssen, R., and Roverso, D. (2019). Intelligent monitoring and inspection of power line components powered by uavs and deep learning. IEEE Power and Energy Technology Systems Journal, 6(1):11-21.

Nwankpa, C., Ijomah, W., Gachagan, A., and Marshall, S. (2018). Activation functions: Comparison of trends in practice and research for deep learning. arXiv preprint arXiv:1811.03378.

Oberweger, M., Wendel, A., and Bischof, H. (2014). Visual recognition and fault detection for power line insulators. In 19th Computer Vision Winter Workshop, Krtiny, Czech Republic, pages 1-8.

Ojala, T., Pietikäinen, M., and Harwood, D. (1996). A comparative study of texture measures with classification based on featured distributions. Pattern recognition, $29(1): 51-59$. 
Olivares Mendez, M. A., Mondragon Bernal, I. F., Campoy Cervera, P., Mejias Alvarez, L., and Martínez Luna, C. V. (2011). Aerial object following using visual fuzzy servoing.

Parunak, H. V. D., Brueckner, S., and Odell, J. J. (2003). Swarming coordination of multiple uav's for collaborative sensing. In Proceedings of Second AIAA" Unmanned Unlimited" Systems, Technologies, and Operations Conference.

Pedregosa, F., Varoquaux, G., Gramfort, A., Michel, V., Thirion, B., Grisel, O., Blondel, M., Prettenhofer, P., Weiss, R., Dubourg, V., Vanderplas, J., Passos, A., Cournapeau, D., Brucher, M., Perrot, M., and Duchesnay, E. (2011). Scikit-learn: Machine learning in Python. Journal of Machine Learning Research, 12:2825-2830.

Pérez-Higueras, N., Caballero, F., and Merino, L. (2018). Learning human-aware path planning with fully convolutional networks. In 2018 IEEE International Conference on Robotics and Automation (ICRA), pages 1-5. IEEE.

Pestana, J., Mellado-Bataller, I., Sanchez-Lopez, J. L., Fu, C., Mondragón, I. F., and Campoy, P. (2014). A general purpose configurable controller for indoors and outdoors gps-denied navigation for multirotor unmanned aerial vehicles. Journal of Intelligent \& Robotic Systems, 73(1-4):387-400.

Pestana, J., Sanchez-Lopez, J. L., Campoy, P., and Saripalli, S. (2013). Vision based gpsdenied object tracking and following for unmanned aerial vehicles. In Safety, security, and rescue robotics (ssrr), 2013 ieee international symposium on, pages 1-6. IEEE.

Pestana, J., Sanchez-Lopez, J. L., De La Puente, P., Carrio, A., and Campoy, P. (2016). A vision-based quadrotor multi-robot solution for the indoor autonomy challenge of the 2013 international micro air vehicle competition. Journal of Intelligent E Robotic Systems, 84(1-4):601-620.

Pfeiffer, M., Schaeuble, M., Nieto, J., Siegwart, R., and Cadena, C. (2017). From perception to decision: A data-driven approach to end-to-end motion planning for autonomous ground robots. In Robotics and Automation (ICRA), 2017 IEEE International Conference on, pages 1527-1533. IEEE.

Phung, M. D., Dinh, T. H., Ha, Q. P., et al. (2019). System architecture for real-time surface inspection using multiple uavs. IEEE Systems Journal.

Polvara, R., Patacchiola, M., Sharma, S., Wan, J., Manning, A., Sutton, R., and Cangelosi, A. (2017). Autonomous quadrotor landing using deep reinforcement learning. arXiv preprint arXiv:1709.03339.

Prasad, P. S. and Rao, B. P. (2016). Condition monitoring of 11 kv overhead power distribution line insulators using combined wavelet and lbp-hf features. IET Generation, Transmission $\&$ Distribution, 11(5):1144-1153. 
Quigley, M., Conley, K., Gerkey, B. P., Faust, J., Foote, T., Leibs, J., Wheeler, R., and Ng, A. Y. (2009). Ros: an open-source robot operating system. In ICRA Workshop on Open Source Software, volume 3.

Quinlan, S. and Khatib, O. (1993). Elastic bands: connecting path planning and control. In Proceedings IEEE International Conference on Robotics and Automation, pages 802-807 vol.2.

Ramirez-Atencia, C., R-Moreno, M. D., and Camacho, D. (2017). Handling swarm of uavs based on evolutionary multi-objective optimization. Progress in Artificial Intelligence, 6(3):263-274.

Redmon, J. and Farhadi, A. (2016). Yolo9000: Better, faster, stronger. arXiv preprint arXiv:1612.08242.

Redmon, J. and Farhadi, A. (2018). Yolov3: An incremental improvement. arXiv preprint arXiv:1804.0276\%.

Ren, S., He, K., Girshick, R., and Sun, J. (2015). Faster r-cnn: Towards real-time object detection with region proposal networks. In Advances in neural information processing systems, pages 91-99.

Rodriguez-Ramos, A., Sampedro, C., Bavle, H., De La Puente, P., and Campoy, P. (2019). A deep reinforcement learning strategy for uav autonomous landing on a moving platform. Journal of Intelligent $\& 3$ Robotic Systems, 93(1-2):351-366.

Rodriguez-Ramos, A., Sampedro, C., Bavle, H., Milosevic, Z., Garcia-Vaquero, A., and Campoy, P. (2017). Towards fully autonomous landing on moving platforms for rotary unmanned aerial vehicles. In 2017 International Conference on Unmanned Aircraft Systems (ICUAS), pages 170-178. IEEE.

Rodriguez-Ramos, A., Sampedro, C., Bavle, H., Moreno, I. G., and Campoy, P. (2018). A deep reinforcement learning technique for vision-based autonomous multirotor landing on a moving platform. In 2018 IEEE/RSJ International Conference on Intelligent Robots and Systems (IROS), pages 1010-1017.

Ronneberger, O., Fischer, P., and Brox, T. (2015). U-net: Convolutional networks for biomedical image segmentation. In International Conference on Medical image computing and computer-assisted intervention, pages 234-241. Springer.

Ross, S., Melik-Barkhudarov, N., Shankar, K. S., Wendel, A., Dey, D., Bagnell, J. A., and Hebert, M. (2013). Learning monocular reactive uav control in cluttered natural environments. In Robotics and Automation (ICRA), 2013 IEEE International Conference on, pages 1765-1772. IEEE.

Rumelhart, D. E., Hinton, G. E., and Williams, R. J. (1986). Learning representations by back-propagating errors. Nature, 323:533-536. 
Sa, I., Hrabar, S., and Corke, P. (2014). Inspection of pole-like structures using a visioncontrolled vtol uav and shared autonomy. In Intelligent Robots and Systems (IROS 2014), 2014 IEEE/RSJ International Conference on, pages 4819-4826. IEEE.

Sampedro, C., Bavle, H., Rodríguez-Ramos, A., Carrio, A., Fernández, R. A. S., SanchezLopez, J. L., and Campoy, P. (2017). A fully-autonomous aerial robotic solution for the 2016 international micro air vehicle competition. In 2017 International conference on unmanned aircraft systems (ICUAS), pages 989-998. IEEE.

Sampedro, C., Bavle, H., Rodriguez-Ramos, A., de la Puente, P., and Campoy, P. (2018a). Laser-based reactive navigation for multirotor aerial robots using deep reinforcement learning. In 2018 IEEE/RSJ International Conference on Intelligent Robots and Systems (IROS), pages 1024-1031. IEEE.

Sampedro, C., Bavle, H., Sanchez-Lopez, J. L., Fernández, R. A. S., Rodríguez-Ramos, A., Molina, M., and Campoy, P. (2016). A flexible and dynamic mission planning architecture for uav swarm coordination. In 2016 International Conference on Unmanned Aircraft Systems (ICUAS), pages 355-363. IEEE.

Sampedro, C., Martinez, C., Chauhan, A., and Campoy, P. (2014). A supervised approach to electric tower detection and classification for power line inspection. In 2014 International Joint Conference on Neural Networks (IJCNN), pages 1970-1977. IEEE.

Sampedro, C., Rodriguez-Ramos, A., Bavle, H., Carrio, A., de la Puente, P., and Campoy, P. (2018b). A fully-autonomous aerial robot for search and rescue applications in indoor environments using learning-based techniques. Journal of Intelligent $\&$ Robotic Systems, pages 1-27.

Sampedro, C., Rodriguez-Ramos, A., Gil, I., Mejias, L., and Campoy, P. (2018c). Imagebased visual servoing controller for multirotor aerial robots using deep reinforcement learning. In 2018 IEEE/RSJ International Conference on Intelligent Robots and Systems (IROS), pages 979-986. IEEE.

Sampedro, C., Rodriguez-Vazquez, J., Rodriguez-Ramos, A., Carrio, A., and Campoy, P. (2019). Deep learning-based system for automatic recognition and diagnosis of electrical insulator strings. IEEE Access, 7:101283-101308.

San Juan, V., Santos, M., and Andújar, J. M. (2018). Intelligent uav map generation and discrete path planning for search and rescue operations. Complexity, 2018.

Sanchez-Lopez, J. L., Fernández, R. A. S., Bavle, H., Sampedro, C., Molina, M., Pestana, J., and Campoy, P. (2016). Aerostack: An architecture and open-source software framework for aerial robotics. In Unmanned Aircraft Systems (ICUAS), 2016 International Conference on, pages 332-341. IEEE.

Sanchez-Lopez, J. L., Molina, M., Bavle, H., Sampedro, C., Fernández, R. A. S., and Campoy, P. (2017a). A multi-layered component-based approach for the development 
of aerial robotic systems: The aerostack framework. Journal of Intelligent $\mathcal{E}$ Robotic Systems, 88(2-4):683-709.

Sanchez-Lopez, J. L., Pestana, J., and Campoy, P. (2017b). A robust real-time path planner for the collision-free navigation of multirotor aerial robots in dynamic environments. In 2017 International Conference on Unmanned Aircraft Systems (ICUAS), pages 316-325. IEEE.

Sanchez-Lopez, J. L., Pestana, J., de la Puente, P., and Campoy, P. (2015). A reliable open-source system architecture for the fast designing and prototyping of autonomous multi-uav systems: Simulation and experimentation. Journal of Intelligent $\&$ Robotic Systems, pages 1-19.

Sanchez-Lopez, J. L., Sampedro, C., Cazzato, D., and Voos, H. (2019). Deep learning based semantic situation awareness system for multirotor aerial robots using lidar. In 2019 International Conference on Unmanned Aircraft Systems (ICUAS), pages 899-908.

Saxena, A., Pandya, H., Kumar, G., Gaud, A., and Krishna, K. M. (2017). Exploring convolutional networks for end-to-end visual servoing. In 2017 IEEE International Conference on Robotics and Automation (ICRA), pages 3817-3823. IEEE.

Scherer, J., Yahyanejad, S., Hayat, S., Yanmaz, E., Andre, T., Khan, A., Vukadinovic, V., Bettstetter, C., Hellwagner, H., and Rinner, B. (2015). An autonomous multi-uav system for search and rescue. In Proceedings of the First Workshop on Micro Aerial Vehicle Networks, Systems, and Applications for Civilian Use, pages 33-38. ACM.

Schlegl, T., Seeböck, P., Waldstein, S. M., Schmidt-Erfurth, U., and Langs, G. (2017). Unsupervised anomaly detection with generative adversarial networks to guide marker discovery. In International Conference on Information Processing in Medical Imaging, pages 146-157. Springer.

Schölkopf, B., Platt, J. C., Shawe-Taylor, J., Smola, A. J., and Williamson, R. C. (2001). Estimating the support of a high-dimensional distribution. Neural computation, 13(7):1443-1471.

Seeböck, P., Waldstein, S., Klimscha, S., Gerendas, B. S., Donner, R., Schlegl, T., Schmidt-Erfurth, U., and Langs, G. (2016). Identifying and categorizing anomalies in retinal imaging data. arXiv preprint arXiv:1612.00686.

Sermanet, P., Eigen, D., Zhang, X., Mathieu, M., Fergus, R., and LeCun, Y. (2013). Overfeat: Integrated recognition, localization and detection using convolutional networks. arXiv preprint arXiv:1312.6229.

Shi, H., Li, X., Hwang, K.-S., Pan, W., and Xu, G. (2016). Decoupled visual servoing with fuzzyq-learning. IEEE Transactions on Industrial Informatics, 14(1):241-252. 
Siddiqui, Z., Park, U., Lee, S.-W., Jung, N.-J., Choi, M., Lim, C., and Seo, J.-H. (2018). Robust powerline equipment inspection system based on a convolutional neural network. Sensors, 18(11):3837.

Siegwart, R. and Nourbakhsh, I. R. (2004). Introduction to Autonomous Mobile Robots.

Silva, W. R. L. d. and Lucena, D. S. d. (2018). Concrete cracks detection based on deep learning image classification. In Multidisciplinary Digital Publishing Institute Proceedings, volume 2, page 489.

Silver, D., Hubert, T., Schrittwieser, J., Antonoglou, I., Lai, M., Guez, A., Lanctot, M., Sifre, L., Kumaran, D., Graepel, T., et al. (2018). A general reinforcement learning algorithm that masters chess, shogi, and go through self-play. Science, 362(6419):11401144.

Silver, D., Lever, G., Heess, N., Degris, T., Wierstra, D., and Riedmiller, M. (2014). Deterministic policy gradient algorithms. In Proceedings of the 31st International Conference on Machine Learning (ICML-14), pages 387-395.

Silver, D., Schrittwieser, J., Simonyan, K., Antonoglou, I., Huang, A., Guez, A., Hubert, T., Baker, L., Lai, M., Bolton, A., et al. (2017). Mastering the game of go without human knowledge. Nature, 550(7676):354.

Simonyan, K. and Zisserman, A. (2014). Very deep convolutional networks for large-scale image recognition. arXiv preprint arXiv:1409.1556.

Srivastava, N., Hinton, G., Krizhevsky, A., Sutskever, I., and Salakhutdinov, R. (2014). Dropout: a simple way to prevent neural networks from overfitting. The journal of machine learning research, 15(1):1929-1958.

Sutskever, I., Martens, J., Dahl, G., and Hinton, G. (2013). On the importance of initialization and momentum in deep learning. In International conference on machine learning, pages 1139-1147.

Sutton, R. S., Barto, A. G., et al. (1998). Introduction to reinforcement learning, volume 2. MIT press Cambridge.

Szegedy, C., Liu, W., Jia, Y., Sermanet, P., Reed, S., Anguelov, D., Erhan, D., Vanhoucke, V., and Rabinovich, A. (2015). Going deeper with convolutions. In Proceedings of the IEEE conference on computer vision and pattern recognition, pages 1-9.

Tai, L., Paolo, G., and Liu, M. (2017a). Virtual-to-real deep reinforcement learning: Continuous control of mobile robots for mapless navigation. In 2017 IEEE/RSJ International Conference on Intelligent Robots and Systems (IROS), pages 31-36.

Tai, L., Paolo, G., and Liu, M. (2017b). Virtual-to-real deep reinforcement learning: Continuous control of mobile robots for mapless navigation. arXiv preprint arXiv:1703.00420. 
Taigman, Y., Yang, M., Ranzato, M., and Wolf, L. (2014). Deepface: Closing the gap to human-level performance in face verification. In Proceedings of the IEEE conference on computer vision and pattern recognition, pages 1701-1708.

Taklaja, P., Oidram, R., Niitsoo, J., and Palu, I. (2013). Main bird excrement contamination type causing insulator flashovers in $110 \mathrm{kv}$ overhead power lines in estonia. Oil Shale, 30(2S):211-224.

Tao, X., Zhang, D., Wang, Z., Liu, X., Zhang, H., and Xu, D. (2018). Detection of power line insulator defects using aerial images analyzed with convolutional neural networks. IEEE Transactions on Systems, Man, and Cybernetics: Systems, pages $1-13$.

Thomas, J., Loianno, G., Sreenath, K., and Kumar, V. (2014). Toward image based visual servoing for aerial grasping and perching. In Robotics and Automation (ICRA), 2014 IEEE International Conference on, pages 2113-2118. IEEE.

Thomas, J., Welde, J., Loianno, G., Daniilidis, K., and Kumar, V. (2017). Autonomous flight for detection, localization, and tracking of moving targets with a small quadrotor. IEEE Robotics and Automation Letters, 2(3):1762-1769.

Tieleman, T. and Hinton, G. (2012). Lecture 6.5-rmsprop: Divide the gradient by a running average of its recent magnitude. COURSERA: Neural Networks for Machine Learning.

Tomic, T., Schmid, K., Lutz, P., Domel, A., Kassecker, M., Mair, E., Grixa, I. L., Ruess, F., Suppa, M., and Burschka, D. (2012). Toward a fully autonomous uav: Research platform for indoor and outdoor urban search and rescue. IEEE robotics \& automation magazine, 19(3):46-56.

Uijlings, J. R., Van De Sande, K. E., Gevers, T., and Smeulders, A. W. (2013). Selective search for object recognition. International journal of computer vision, 104(2):154171.

Vapnik, V. N. (1999). An overview of statistical learning theory. IEEE transactions on neural networks, 10(5):988-999.

Varghese, A., Gubbi, J., Sharma, H., and Balamuralidhar, P. (2017). Power infrastructure monitoring and damage detection using drone captured images. In 2017 International Joint Conference on Neural Networks (IJCNN), pages 1681-1687. IEEE.

Verbeke, J. and Schutter, J. D. (2018). Experimental maneuverability and agility quantification for rotary unmanned aerial vehicle. International Journal of Micro Air Vehicles, 10(1):3-11.

Vincent, P. and Rubin, I. (2004). A framework and analysis for cooperative search using uav swarms. In Proceedings of the 2004 ACM symposium on Applied computing, pages 79-86. ACM. 
Waharte, S. and Trigoni, N. (2010). Supporting search and rescue operations with uavs. In Emerging Security Technologies (EST), 2010 International Conference on, pages 142-147. IEEE.

Wang, X. and Zhang, Y. (2016). Insulator identification from aerial images using support vector machine with background suppression. In 2016 International Conference on Unmanned Aircraft Systems (ICUAS), pages 892-897. IEEE.

Wang, Y., Sun, S., and Zhong, J. (2016). An ensemble anomaly detection with imbalanced data based on robot vision. Int. J. Rob. Autom, 31(2).

Wei, Y., Blake, M. B., and Madey, G. R. (2013). An operation-time simulation framework for uav swarm configuration and mission planning. Procedia Computer Science, 18:1949-1958.

Xie, L., Wang, S., Markham, A., and Trigoni, N. (2017). Towards monocular vision based obstacle avoidance through deep reinforcement learning. arXiv preprint arXiv:1706.09829, RSS workshop New Frontiers for Deep Learning in Robotics.

Yang, G.-Z., Bellingham, J., Dupont, P. E., Fischer, P., Floridi, L., Full, R., Jacobstein, N., Kumar, V., McNutt, M., Merrifield, R., Nelson, B. J., Scassellati, B., Taddeo, M., Taylor, R., Veloso, M., Wang, Z. L., and Wood, R. (2018). The grand challenges of science robotics. Science Robotics, 3(14).

Zhai, Y., Chen, R., Yang, Q., Li, X., and Zhao, Z. (2018). Insulator fault detection based on spatial morphological features of aerial images. IEEE Access, 6:35316-35326.

Zhai, Y., Wang, D., Zhang, M., Wang, J., and Guo, F. (2017). Fault detection of insulator based on saliency and adaptive morphology. Multimedia Tools and Applications, 76(9):12051-12064.

Zhang, J., Springenberg, J. T., Boedecker, J., and Burgard, W. (2017). Deep reinforcement learning with successor features for navigation across similar environments. In 2017 IEEE/RSJ International Conference on Intelligent Robots and Systems (IROS), pages $2371-2378$.

Zhao, Z., Xu, G., Qi, Y., Liu, N., and Zhang, T. (2016). Multi-patch deep features for power line insulator status classification from aerial images. In 2016 International Joint Conference on Neural Networks (IJCNN), pages 3187-3194. IEEE.

Zhu, Y., Mottaghi, R., Kolve, E., Lim, J. J., Gupta, A., Fei-Fei, L., and Farhadi, A. (2017). Target-driven visual navigation in indoor scenes using deep reinforcement learning. In Robotics and Automation (ICRA), 2017 IEEE International Conference on, pages 3357-3364. IEEE.

Zitnick, C. L. and Dollár, P. (2014). Edge boxes: Locating object proposals from edges. In European conference on computer vision, pages 391-405. Springer. 